\title{
Berufs- und \\ wirtschaftspädagogische Analysen Aktuelle Forschungen zur beruflichen Bildung
}

Uwe Faßhauer, Bärbel Fürstenau,

Eveline Wuttke (Hrsg.)

Schriftenreihe der Sektion Berufs- und Wirtschaftspädagogik der Deutschen Gesellschaft für Erziehungswissenschaft

DGE Deutsche Gesellschatt 
Schriftenreihe der Sektion

Berufs- und Wirtschaftspädagogik

der Deutschen Gesellschaft für

Erziehungswissenschaft (DGfE) 
Uwe Faßhauer

Bärbel Fürstenau

Eveline Wuttke (Hrsg.)

Berufs- und wirtschaftspädagogische Analysen - aktuelle Forschungen zur beruflichen Bildung

Verlag Barbara Budrich

Opladen • Berlin • Toronto 2012 
Bibliografische Information der Deutschen Nationalbibliothek

Die Deutsche Nationalbibliothek verzeichnet diese Publikation in der Deutschen

Nationalbibliografie; detaillierte bibliografische Daten sind im Internet über

http://dnb.d-nb.de abrufbar.

Alle Rechte vorbehalten.

C 2012 Verlag Barbara Budrich, Opladen, Berlin \& Toronto

www.budrich-verlag.de

ISBN 978-3-8474-0007-3 (Paperback)

eISBN 978-3-86649-549-4 (eBook)

Das Werk einschließlich aller seiner Teile ist urheberrechtlich geschützt. Jede Verwertung außerhalb der engen Grenzen des Urheberrechtsgesetzes ist ohne Zustimmung des Verlages unzulässig und strafbar. Das gilt insbesondere für Vervielfältigungen, Übersetzungen, Mikroverfilmungen und die Einspeicherung und Verarbeitung in elektronischen Systemen.

Umschlaggestaltung: bettina lehfeldt graphic design, Kleinmachnow Typographisches Lektorat: Ulrike Weingärtner, Textakzente, Gründau 
Vorwort

\section{Teil I: Disziplinäre Reflexionen}

Rolf Dubs

Überlegungen zum Impact pädagogischer Forschungen

Uwe Elsholz

Betriebliche Weiterbildung als interdisziplinäres

Forschungsfeld - Annäherung an eine berufs- und

wirtschaftspädagogische Perspektive

Georg Tafner

Reflexive Wirtschaftspädagogik:

Wie Ethik, Neo-Institutionalismus und Europädagogik

neue Perspektiven eröffnen könnten

\section{Teil II: Lehr-/Lernforschung in der beruflichen Bildung}

Markus Ammann

Betriebspraktika unter dem Aspekt der

Arbeitszufriedenheit - eine kritische Auseinandersetzung

Carmela Aprea et al

Digitale Technologien als Tools zur Förderung der

Konnektivität des Lernens in Schule und Betrieb

Kristina Kögler, Eveline Wuttke

Unterrichtliche Monotonie als Bedingungsfaktor für

Schülerlangeweile im Fach Rechnungswesen 75 


\section{Jeannine Ryssel}

Die Lernwirksamkeit von einfachem und elaboriertem

Feedback in Verbindung mit dem Erstellen von

Concept Maps im Planspielunterricht

\section{Teil III: Professionalisierung des Personals in der beruflichen Bildung}

Alexandra Dehmel

Lehrerausbildung für den berufsbildenden Bereich in

Deutschland und England - ausgewählte Ergebnisse einer komparativen Studie

Stephan Kösel

Triadengespräche zur Rekonstruktion didaktischer

Überzeugungen als Bestandteil berufspädagogischer

Professionalität

Birgit Lehmann

Entwicklung eines Instruments zur Erfassung unterrichtsbezogener Metaphern

Sandra Trost

Erfolgreich Studieren - Entwicklung und Validierung eines Fragebogens zur Erfassung studienbezogener Selbstregulationsprozesse

\section{Teil IV: Berufliche Qualifizierung und Weiterbildung}

Julia K. Müller, Rita Meyer

Lernen und Arbeiten in Balance? Vereinbarkeitsstrategien von Beschäftigten in wissensintensiven Branchen 


\section{Franz Kaiser}

Was kennzeichnet Kaufleute? - Ihr berufliches Denken und

Handeln aus historischer, soziologischer und

ordnungspolitischer Perspektive

Petra F. Köster

Kompetenzentwicklung und organisationale Veränderung

am Beispiel von Festivalveranstaltern

Martin Kröll

Karrieren und Weiterbildung von Ingenieuren

Lars Windelband, Georg Spöttl

Diffusion von Technologien in die Facharbeit und deren

Konsequenzen für die Qualifizierung am Beispiel des

„Internet der Dinge“

Verzeichnis der Autorinnen und Autoren 



\section{Vorwort}

Den Stellenwert und die Bedeutung berufs- und wirtschaftspädagogischer Analysen zur beruflichen Bildung immer wieder neu - und durchaus auch kritisch - zu hinterfragen ist fester und kontinuierlicher Bestandteil der disziplinären Reflexion und Selbstvergewisserung. Begründet liegt dies nicht zuletzt in den Wandlungsprozessen der zentralen Gegenstandsbereiche der BWP und den Abgrenzungen gegenüber „Nachbardisziplinen“, die sich ebenfalls - und verstärkt - mit der beruflichen Bildung und dem beruflichen Lernen befassen. Im aktuellen Kontext der weiteren organisationalen Ausdifferenzierung der empirischen Bildungsforschung außerhalb er DGfE sind Impulse zur Diskussion der Wirkung pädagogischer, bildungswissenschaftlicher insbesondere aber berufs- und wirtschaftspädagogischer Forschungen wichtig und notwendig. Die Herbsttagung der Sektion Berufs- und Wirtschaftspädagogik, die 2012 in Konstanz stattgefunden hatte, zeigte nicht nur die große Bandbreite aktueller berufs- und wirtschaftspädagogischer Analysen. Diese wurden, nicht zuletzt mit Bezug auf die Keynote von Prof. Rolf Dubs, auch im Sinne disziplinärer Reflexionen in den Diskurs aufgenommen. Deutlich wurde erneut das für die BWP typische forschungsmethodische Spektrum in qualitativen und quantitativen Untersuchungsdesigns.

Der hier vorgelegte Band versammelt Beiträge dieser Tagung, die aus einem Reviewverfahren hervorgegangen sind. Zu Beginn werden in der angesprochenen Keynote sowie in zwei Beiträgen Aspekte des wissenschaftlichen Selbstverständnisses der Berufs- und Wirtschaftspädagogik diskutiert.

Im zweiten Abschnitt stehen berufs- und wirtschaftspädagogische Analysen zur Lehr-/Lernforschung in der beruflichen Bildung. Neben Untersuchungen zur Lernwirksamkeit von unterschiedlichen Formen des Feedbacks und den Bedingungsfaktoren von Langeweile bei Lernenden treten mit der Lernortkooperation bzw. den Betriebspraktika die Rahmenbedingungen beruflichen Lernens in den Fokus.

Der dritte Abschnitt befasst sich mit dem für die BWP ebenso zentralen Bereich der Professionalisierung des Personals in der beruflichen Bildung. Die Untersuchung von didaktischen Überzeugungen sowie instruktionalen Mustern von Lehrpersonen schließt dabei durchaus an die Lehr-/Lernforschung an. $\mathrm{Zu}$ diesem Abschnitt zählt auch eine der wenigen, und hier auch sehr aufschlussreichen, international vergleichenden Studien zur Ausbildung von Lehrenden an beruflichen Schulen.

Der vierte Abschnitt versammelt Beiträge zur beruflichen Qualifizierung in dem relativ neuen Segment der Festivalveranstalter, zur Berufsforschung bei Kaufleuten bzw. der Entwicklung von Facharbeit unter dem Aspekt der Diffusion von Technologien. Abgerundet wird dieser Abschnitt mit eher berufsbiografisch orientierten Studien zur Weiterbildung von Ingenieuren und 
den Strategien zur Vereinbarung von Lernen und Arbeiten in wissensintensiven Branchen.

Der Vorstand der Sektion Berufs- und Wirtschaftspädagogik der Deutschen Gesellschaft für Erziehungswissenschaft bedankt sich sehr herzlich bei den Ausrichtern, Prof. Dr. Thomas Deißinger, Prof. Dr. Jürgen Seifried sowie Prof. Dr. Sabine Hochholdinger.

Für die Herausgeberschaft Uwe Faßhauer Bärbel Fürstenau Eveline Wuttke

Schwäbisch Gmünd, März 2012 


\title{
Überlegungen zum Impact der pädagogischen Forschung
}

\author{
Rolf Dubs
}

\section{Die Nutzung von Erkenntnissen der pädagogischen Forschung im Alltag}

Drei Beispiele sollen sensibilisieren. In einer Bildungsverwaltung ${ }^{1}$ wurde die Weisung erlassen, auf der Volksschulstufe keine Hausaufgaben mehr zu erteilen. Auf eine angeforderte Begründung wurde mitgeteilt, dass eine Mehrzahl der empirischen Untersuchungen zur Wirksamkeit von Hausaufgaben keine positive Wirkung nachweisen, ein Sachverhalt, der an sich zutreffend ist. Auf die Frage, ob in den dem Entscheid zugrunde gelegten Studien analysiert worden sei, welche Typen von Hausaufgaben in welcher Form erteilt wurden, gab es keine Antwort. Damit hat man aber die empirischen Untersuchungen unsorgfältig analysiert. Entscheidend für die Lernwirksamkeit von Hausaufgaben ist nicht, ob solche erteilt, sondern in welcher Form sie erteilt werden. Demzufolge sind die vielen empirischen Untersuchungen, welche die Form der Aufgabenerteilung nicht kontrollieren, unbrauchbar und lassen für den Schulalltag keine gesicherten Schlüsse zu. Leider werden - wie dieses Beispiel zeigt - in der Schulpraxis aus der wissenschaftlichen Forschung immer wieder Folgerungen gezogen und Maßnahmen als ,absolute Wahrheit" umgesetzt, oft weil die Forschenden in ihren Untersuchungen wichtige Einflussgrößen aus untersuchungstechnischen Gründen oder mangels genügender Einsicht in die Praxis nicht erfassen und ihre Erkenntnisse nicht deutlich genug relativieren. Besonders fragwürdig wird es dort, wo für bildungspolitische Entscheidungen solche Untersuchungen zitiert werden, welche eine bestimmte politische oder eine vorgefasste Meinung bestätigen. In der Schweiz ließen sich etwa im Bereich der Gliederung des Schulsystems Gutachten finden, welchen dieses fragwürdige Verhalten zugrunde liegt.

Das zweite Beispiel betrifft mich selbst. Als ich an der zweiten Auflage meines Buches „Lehrerverhalten“ (Dubs 2009) arbeitete, in welchem ich erneut versuchte, gesicherte empirische Erkenntnisse zu Handlungsempfehlungen für den Unterricht abzuleiten, kamen mir Zweifel auf, ob ich überhaupt noch eine zweite Auflage auflegen soll, nachdem ich erkennen

1 Kritische Beispiele werden in diesem Beitrag nicht formell zitiert, weil es nicht meinem Verständnis entspricht, Personen ,anzugreifen“. Wichtiger sind mir die Lehren, die gezogen werden sollten. 
musste, dass die Ergebnisse aus Untersuchungen selbst zu Problemen, bei denen vor zehn Jahren noch eine Trendaussage möglich war, immer widersprüchlicher wurden. Dies nicht zuletzt, weil sich die Forschungsmethoden verfeinert haben. Deshalb ist auch verständlich, dass immer mehr Studien mit dem Hinweis ,more research is needed“ enden. Aber was ist dann der Impact der Forschung für die Qualitätsverbesserung der Schule?

Ein drittes Beispiel betrifft die Auswahl von Forschungsprojekten und Finanzierungspolitik der forschungspolitischen Gremien. Ein DFG-Projekt mit vielen Einzelstudien brachte interessante Erkenntnisse, die mit darauf abgestimmten Materialien für Lehrpersonen und Lernende dem alltäglichen Unterricht hätten dienstbar gemacht werden können. Alle Versuche zur Fortsetzung des Projekts scheiterten, weil einerseits die forschungspolitischen Gremien der Auffassung waren, die Entwicklung und Überprüfung von Lernmaterialien seien keine wissenschaftlichen Leistungen und nicht wenige Projektleitende ihr Desinteresse an Umsetzungsmaßnahmen bekundeten bzw. ihre geringe Kompetenz in unterrichtspraktischen Fragen ausdrückten. Wo liegt dann aber der Impact der empirischen Forschung?

Angesichts der Krise der Staatshaushalte ist mit Kürzungen der Forschungsbeiträge zu rechnen. Spätestens dann gewinnt vor allem aus politischer Sicht die Frage des Impacts der Forschung höchste Bedeutung. Die Entwicklung in England mit den Kürzungen der Beiträge für die Geisteswissenschaften sollte ein mahnendes Beispiel sein. Deshalb muss sich die pädagogische Forschung viel intensiver mit dem Impact auseinandersetzen. Die Zielrichtung ist auf die Frage auszurichten, was sie konkret zur nachhaltigen Innovation und Qualitätsverbesserung der Schulen beiträgt, wobei angesichts der vielen gesellschaftlichen und sozialen Fehlentwicklungen die Schulqualität nicht nur unter dem Gesichtspunkt der kognitiven Leistung zu verstehen, sondern von einem erweiterten Qualitätsbegriff (vielgestaltige Lernleistungen, Werterziehung, Zufriedenheit, Integration usw.) auszugehen ist. Dieser Beitrag möchte zur Reflexion über den Impact der empirischen pädagogischen Forschung mit Bezug auf ihre Praxisrelevanz anregen.

\section{Der erste Problemkreis: Pendelschläge und Slogans}

Die Schule braucht Innovationen. Längerfristig wirksam sind sie jedoch nur, wenn sie nicht nur punktuell betrachtet, sondern in Beziehung zu den gesamten Rahmenbedingungen der Schule gebracht werden, forschungsmäßig einigermaßen gesichert sind (Trendaussagen der bisherigen Forschung nicht völlig vernachlässigt werden), und sie nicht nur kurzfristigen politischen Strömungen sowie Modeerscheinungen aller Art folgen. Viele empirische Untersuchungen, welche diese Bedingungen nicht erfüllen, führen - gar 
wenn sie überinterpretiert werden - zu Pendelschlägen, welche die Lehrerschaft und die Eltern zunehmend mehr verunsichern. Besonders kritisch wird es, wenn die Erziehungswissenschaften mit ihren Erkenntnissen solche Pendelschläge verstärken, weil sie selbst - und nicht selten als Folge momentan vorherrschender pädagogischer Paradigmen (z.B. Konstruktivismus, Modularisierung, Lernfelder) - einseitige oder atomistische Einflüsse auf die Schule haben. Kritisch wird es auch dort, wo zugleich mit vieldeutigen Schlagwörtern (z.B. Chancengleichheit) operiert wird. Wie Komisar/ McCellan (1968) nachweisen, sind pädagogische Neuerungen dann prinzipiell am leichtesten zu verbreiten, wenn dafür ein Slogan verwendet wird, der vieldeutig ist, aber breiten Kreisen - wenn auch mit ganz unterschiedlichen Zielvorstellungen gefällt. Schwierig wird es erst dann, wenn der Slogan umgesetzt werden soll. Dann entstehen unlösbare Widersprüche zwischen Interessengruppen infolge der Vieldeutigkeit des Slogans. Leider verstärken sich Pendelschläge und Slogans gegenseitig: Man erkennt ein Problem beim gegenwärtigen Zustand der Schule. Die Kritik führt zu einem Slogan, der einen Pendelschlag in die Richtung des Slogans einleitet. Findet man schliesslich eine Lösung, wird wie immer bei Schulfragen - bald erkannt, dass auch sie Nachteile hat. Dann entsteht häufig ein neuer Slogan und das Pendel schlägt wieder auf die Gegenseite aus. Beispiele dafür sind etwa der Konstruktivismus (Mitte der neunziger Jahre war man als Forschender in den Vereinigten Staaten geradezu ein Aussenseiter, wenn man seine Arbeit nicht mit dem Konstruktivismus in Verbindung brachte), die Modularisierung der Lehrpläne (mit den vielen definitorischen Unklarheiten nicht zuletzt an den Universitäten), die Kompetenzorientierung des Unterrichts (wobei immer unklarer wird, wie Bildungsstandards und Kompetenzen aufeinander abgestimmt werden und oft ein Rückfall in die Lernzielbewegungen der siebziger Jahre zu beobachten ist) (siehe dazu Dubs 2006).

Angesichts der negativen Folge von unbedachten modischen Pendelschlägen muss sich die pädagogische Forschung um differenzierte und nachhaltige Innovationen bemühen, indem sie nie vorgibt, dass Neues bestehende Probleme für immer beseitigt. Sie muss bei der Propagierung des Neuen immer auch mögliche neue Probleme und Erscheinungen offen darlegen, um dogmatische Entwicklungen und neue Einseitigkeiten zu verhindern, gar wenn zu erwarten ist, dass Lehrpersonen die neuen Erkenntnisse überinterpretieren und auf ihre eigenen Vorstellungen umdeuten. Als Beispiel dazu sei an den Konstruktivismus und an die vielen Fehlinterpretationen konstruktivistischen Unterrichtens (z.B. konstruktivistisches Lernen sei nur im Gruppenunterricht möglich) oder an die vielen Interpretationen von Kompetenzen erinnert. Man täte gut daran, sich stärker an Sprangers Gesetz der ungewollten Nebenwirkungen zu orientieren, welches besagt, dass es in der Pädagogik nichts gibt, was nicht auch wieder Nachteile hat. Im Weiteren müssen an die Stelle von Slogans eindeutig definierte Begriffe und unmissverständliche 
Operationalisierungen treten, welche weder die Schulpraxis irreleiten noch geeignet sind, Hypothesenprüfungen durch unklare Begriffswelten zu eigenen Gunsten zu interpretieren. Ein gegenwärtig typisches Beispiel dafür sind Untersuchungen zum Führungsstil im Schulleitungshandeln.

\section{Der zweite Problemkreis: Die Offenlegung der Werte}

Bei der Interpretation und Verwendung von empirischen Forschungsergebnissen ergeben sich bei Politikern und Lehrpersonen viele Schwierigkeiten, weil viele Forschende ihre Werthaltungen und bildungspolitischen Zielvorstellungen nicht offen legen oder wenigstens bekannt machen, welche Werte einer einzelnen Studie zugrunde liegen, damit der Stellenwert der Erkenntnisse im Zusammenhang der gesamtschulischen Betrachtungsweise erkennbar wird. Wenn beispielsweise die Lernleistungen im Mittelpunkt einer Untersuchung stehen, sind die Ergebnisse anders zu gewichten, als wenn es um Integrationsbemühungen geht. Insbesondere in der Forschung um die Schulorganisation (gegliederte oder integrierte Schulsysteme) entstehen immer wieder Widersprüche in den Ergebnissen von systematischen Untersuchungen, weil die bildungspolitischen Annahmen zu wenig transparent sind. Als Folge davon äußern sich dann bei politischen Auseinandersetzungen selbst renommierte Forschende in der Öffentlichkeit widersprüchlich, weil sich ihre Forschungen auf streng abgegrenzte Gebiete beschränken (z.B. auf die Wirkungen der kognitiven Leistungen), sie aber bei öffentlichen Stellungnahmen nicht ganzheitlich argumentieren und sich dabei in Widersprüche verwickeln. Die Folge davon ist eine große Verunsicherung in der politischen Entscheidungsfindung.

Deshalb müssen sich empirisch Forschende viel stärker mit bildungsphilosophischen Fragen beschäftigen, um den Stellenwert ihrer Untersuchungen für die politische und schulpraktische Umsetzung besser zu artikulieren; vielleicht aber auch, um ihre Forschungsvorhaben etwas ganzheitlicher zu sehen.

\section{Der dritte Problemkreis: Die Untersuchungsgegenstände und Hypothesen}

Es will mir scheinen, dass der unheilvolle Publikationsdruck, unter dem die jungen Wissenschaftlerinnen und Wissenschaftler stehen, die Atomisierung der empirischen Forschung negativ zu beeinflussen beginnt. Unter diesem 
Druck entwickeln die Forschenden nicht selten Hypothesen, welche Probleme aufwerfen, die Praktiker aus ihrer Erfahrung ohne Weiteres und verlässlich beantworten können. Dazu wählen sie einfache Forschungsmethoden wie Befragungen ohne daran zu denken, dass überall dort, wo Lehrpersonen oder Schulleitungsmitglieder eigenes Verhalten oder erzielte Fortschritte zu beurteilen haben, ohnehin nur positive Erkenntnisse resultieren. Und sehr häufig werden bei solchen Untersuchungen die Rahmenbedingungen (intervenierende Variablen) vernachlässigt, wobei mit dem Einsatz von immer differenzierteren Statistiken, die Wissenschaftlichkeit betont wird, wenn auch alles mehr und mehr zum Selbstzweck wird. Schließlich enden solche Untersuchungen oft bloß mit Bestandesaufnahmen ohne Schlussfolgerungen für die Schule. Beispiele dazu gibt es viele. So „bestätigen“ solche Erhebungen den Erfolg von Schulentwicklungsarbeiten aus der Sicht der Schulleitungspersonen und der Lehrkräfte immer wieder, obschon die Wirkungsforschung bislang sehr bescheidene Auswirkungen der Schulentwicklung auf die Qualität einer Schule zeitigt.

Soll der Impact der empirischen Bildungsforschung erhöht werden, muss der äußere Druck auf Forschende, nur anhand ihrer Publikationen beurteilt zu werden, entfallen. Es führt zu vielen wenig nützlichen, wenig differenzierten Forschungsvorhaben, wodurch sich die Widersprüche aus der empirischen Forschung verstärken. Ganz generell anzustreben ist ein „Design-BasedImplementation Research" (Penuel et al. 2011), in welchem (1) das Augenmerk stärker auf bildungspraktische Probleme aus einer multidisziplinären Sicht aller Stakeholder der Schule (Politiker, Administratoren, Lehrpersonen, Forschende) zu belegen ist, (2) die Hypothesen nicht top-down von den Forschenden allein vorgegeben werden, sondern sie zusammen mit allen Stakeholdern entworfen werden und (3) immer das Ziel einer Innovation oder einer Qualitätsverbesserung der Schule angestrebt wird. Die empirische Bildungsforschung muss $\mathrm{zu}$ einer Gemeinschaftsaufgabe aller Stakeholder auf der Makro- und der Mikroebene werden. Wie nötig dies ist, sei an einem Beispiel belegt: In letzter Zeit werden auch im deutschsprachigen Bereich viele Untersuchungen über die Führung von Schulen durchgeführt. Leider beziehen aber die Forschenden die Schulaufsicht, die Schuladministration und die Lehrkräfte weder bei der Hypothesenbildung noch beim Versuchsdesign in die Vorbereitung und Durchführung ihrer Untersuchungen ein. Dadurch kann einerseits nicht deutlich gemacht werden, welchen Einfluss die konkreten administrativen Rahmenbedingungen auf die Ergebnisse insbesondere bei länderübergreifenden Studien haben; und andererseits wird den Vertretenden aus der Administration nur selten bewusst, welchen Einfluss staatliche Rahmenbedingungen auf die Ergebnisse aus der Schulführungsforschung haben. Auch erkennen die Verwaltungspersonen nie bewusst, wie bedeutsam eine klare Definition der Teilautonomie ist. Die selbständige Schule ist bereits zu einem gefährlichen Slogan geworden. 


\section{Der vierte Problemkreis: Die zunehmenden Widersprüche aus Ergebnissen der empirischen Forschung}

Selbstverständlich wird die empirische Forschung zu einem pädagogischen Problembereich immer über längere Zeit widersprüchliche Ergebnisse vorlegen. Im Interesse des Impacts für die Bildungspraxis sollte jedoch jedes Forschungsprojekt so gestaltet werden, dass es wenigstens eine Trendaussage zulässt und so sorgfältig geplant und durchgeführt wird, dass nicht im Voraus vermeidbare Widersprüche auftreten. Dazu sollten die folgenden Aspekte beachtet werden:

(1) Die Forschenden sollten bei jedem Vorhaben, die Werte, die sie ihrer Forschung zugrunde legen, aus einer ganzheitlichen bildungspolitischen und/oder schulischen Sicht darlegen und begründen und allfällige Begrenzungen ihrer Erkenntnisse für die Schulpraxis darlegen, um Übergeneralisierungen und Fehlentwicklungen von Praktikern zu verhindern.

(2) Beim Entwurf von Hypothesen sollten alle Stakeholder beigezogen werden, damit die Hypothesen für die Schulwirklichkeit relevant sind. Besondere Vorsicht ist mit der üblich gewordenen Zusammenfassung der bisherigen Forschungsergebnisse als Ausgangspunkt für neue Hypothesen geboten. Solche Zusammenfassungen ,gaukeln“ oft vor, sie erbrächten „objektive“ Erkenntnisse und stellten die beste Grundlage für neue und wertvolle Hypothesen dar. In vielen Fällen führen solche Hypothesen jedoch in immer feinere Details perfekter Forschung, die aber wenn auch wissenschaftlich vielleicht interessant - atomistischer werden und sich von den Bedürfnissen der Schulpraxis weiter entfernen, weil die Rahmenbedingungen nicht mehr genügend genau differenziert werden.

(3) Die Rahmenbedingungen werden überhaupt immer mehr zur Krux der empirischen Forschung. Unsorgfältig definierte und erfasste Rahmenbedingungen erbringen selbst bei guten Hypothesen immer wieder Widersprüche. Alle Rahmenbedingungen zu erfassen ist unmöglich. Deshalb bleibt die empirische Forschung immer reduktionisiert. Daher sollte die Begrenzung der Forschungsresultate aufgrund von Rahmenbedingungen in jedem Fall angesprochen werden. Besondere Vorsicht ist mit internationalen Vergleichsstudien geboten, denn sie tragen interkulturellen Eigenarten und verschiedenartigen Rahmenbedingungen zu wenig Rechnung und führen zu häufig zu Übergeneralisierungen.

(4) Mehr Beachtung ist Mehr-Ebenen-Betrachtungen $\mathrm{zu}$ schenken, indem nicht nur untersucht wird, wie sich Sachverhalte und Zusammenhänge auf der Ebene der Einzelschule mit ihrem Unterricht darstellen. Zu über- 
prüfen ist immer, wie der Untersuchungsgegenstand aus der Sicht der Bildungspolitik und der Schuladministration zu beurteilen ist, um ganzheitliche Stimmigkeit zu erreichen. Ergebnisse aus der Innovationsforschung beispielsweise können je nach den Gegebenheiten auf der Behördenebene ganz unterschiedlich ausfallen, selbst wenn in einzelnen Schulen oder im Unterricht die genau gleichen Prozesse ablaufen. So erklären unterschiedliche Arbeitsbedingungen und Entlastungsmöglichkeiten für Lehrkräfte oder bereitgestellte Lernmaterialien sowie eine gezielte Lehrerweiterbildung die Unterschiede im Erfolg mit einer Innovation.

(5) Als kritisch zu betrachten sind noch so sorgfältige Meta-Analysen, weil sie die Rahmenbedingung nicht mit genügender Differenzierung betrachten. Und geradezu gefährlich ist die neuere Tendenz, Daten zu analysieren, welche die Forschenden nicht selbst erhoben haben. Zwar lassen sich immer interessante statistische Zusammenhänge entdecken, welche aber infolge ungenügender Beachtung der konkreten Rahmenbedingungen sowie der Auswertungsverfahren stets zu Fehlschlüssen verleiten.

(6) Seit TIMSS und PISA lässt sich in der Lehr-Lern-Forschung immer häufiger eine Beschränkung auf Fachbereiche erkennen, in welchen mittels Tests die Daten einfach zu erheben sind, was meistens zu einer Reduktion der zu betrachtenden Unterrichtsfächer führt und Ergebnisse bringt, die nicht auf andere Fächer übertragbar, trotzdem aber so generalisiert werden, dass sich sehr viele Widersprüche ergeben. Der Trend vieler Forschenden, vor allem aus der Bildungstheorie und der pädagogischen Psychologie, die Fachdidaktik zu vernachlässigen, könnte $\mathrm{zu}$ vielen Fehlschlüssen der empirischen Forschung in der Lehr-Lern-Theorie führen.

An sich ist die Wende zur empirischen Forschung ein Glücksfall. Ohne sie bliebe Vieles in den Erziehungswissenschaften völlig ungeklärt und in der Zeit der Massenmedien würden Slogans die Pädagogik zunehmend stärker und wenig bedacht beeinflussen. Ein Impact ist aber erst gegeben, wenn sich die empirische Forschung erfolgreich bemüht, Widersprüche zu vermindern. Bedingungen dafür sind: Design-Based-Implementation Research, Studien, welche unterschiedlichen Rahmenbedingungen Rechnung tragen und von wirklichkeitsnahen Hypothesen ausgehen. 


\section{Der fünfte Problemkreis: Die Rolle der staatlichen Träger der Bildungspolitik}

Es wäre interessant zu untersuchen, welchen Einfluss die staatlichen Träger der Bildungspolitik auf die empirische Forschung haben. Aus einer nahezu fünfzigjährigen Erfahrung lassen sich wenigstens hypothetisch einige Anmerkungen vortragen. Für die Bildung zuständige staatliche Stellen unterstützten die empirische Forschung (vor allem die Schulversuchsforschung) nicht aufgrund wissenschaftlicher Bedürfnisse oder Kriterien. Sehr oft erteilen sie Aufträge aufgrund von bildungspolitischen Slogans, die bei der Politik Anklang finden und/oder von dort her zur Umsetzung gefordert werden. Nicht selten beauftragen sie Forschende, um ihre eigenen bildungspolitischen Ideen wissenschaftlich gestützt durchsetzen zu können. Leider spielen aber auch gleiche politische Ideen sowie Sympathie und Antipathie zu Forschenden mit ihren Ansätzen mit, wenn immer wieder die gleichen Forschenden beauftragt oder nicht zur Kenntnis genommen werden. Allerdings sind dafür Forschende auch selbstverantwortlich. Negativ wirken sich wissenschaftliche Überheblichkeit, Desinteresse an schulpraktischen Fragen und eine unverständliche Sprache aus. Dazu kommt, dass wissenschaftliche Gremien, welche über Forschungsgesuche entscheiden, nicht selten „Seilschaften“" gleicher wissenschaftstheoretischer Positionen bilden, denen junge Forschende oft ausgeliefert sind.

Eine wegweisende Studie legte Stamm vor (2003). Sie untersuchte, welche Ziele die Auftraggeber von Schulversuchen anstreben, und was die Ergebnisse sind. Aus achtzehn schweizerischen Schulversuchen ergab sich folgendes Bild: Vier Studien bezeichnete sie als Reaktion. Es lag eine Evaluation zur Kontrolle der Wirkungen und zur Legitimation des Neuen vor. Die Nutzung der wissenschaftlichen Erkenntnisse war nachweisbar. Bei sechs Studien lag eine Blockade vor. Die Nutzung der Erkenntnisse war unklar oder nicht vorhanden. Drei Studien hatten Alibi-Charakter, indem sie nur der Legitimation der Innovation dienten und keine klare Nutzung der Erkenntnisse erkennbar war. Bei fünf Studien erbrachte die Evaluation, dass der Versuch entwicklungs- und umsetzungsorientierten Charakter hatte. Diese Studie zeigt deutlich, wie wenig differenziert viele Schulbehörden mit wissenschaftlichen Studien umgehen können. Die Ergebnisse sind weder für Schulbehörden noch für die empirische Forschung besonders überzeugend. Einerseits zeigen sie, wie die Forschung von Behörden missbraucht werden kann, und andererseits verweisen sie auf eine gewisse Hilflosigkeit von Forschenden gegenüber bildungspolitischen Missbräuchen ihrer Forschungsergebnisse. Um auf solche Weise den Impact der empirischen Forschung nicht zu vermindern, sollten in der Beziehung Auftraggeber/Forschende die folgenden Prinzipien zugrunde gelegt werden: Werte und politische Ziele, die einem 
Vorhaben zugrunde gelegt werden, sind transparent zu machen und Strategien der Implementation des Vorhabens sind in jedem Fall in die Studien einzubeziehen. Forschende sollten nie Gefälligkeitsaufträge annehmen und die Analyse der bereits bekannten Forschungsergebnisse nicht zur Stützung der eigenen Auffassung filtriert interpretieren, ein Sachverhalt der sich bei Gutachten zu umstrittenen bildungspolitischen Problemen immer wieder beobachten lässt (insbesondere bei Gutachten über die Gestaltung des Schulsystems, Chancengleichheit, Führungskonzeption der selbständigen Schule, mediengestütztes eigenständiges Lernen). $\mathrm{Zu}$ warnen ist vor allem vor dem Bestreben, alles als „objektiv“ richtig zu bezeichnen. Wichtiger wäre es, die eigene Meinung - selbst wenn sie mit Trendaussagen gestützt ist - begründet darzustellen. Auseinandersetzungen über Gutachten oder Modellversuche, die nicht transparent ausgetragen werden, schaden vor allem bei bildungspolitischen Entscheidungen der Glaubwürdigkeit der empirischen Forschung und damit dem Impact.

\section{Der sechste Problemkreis: Der Transfer der Erkenntnisse der empirischen Forschung in die Schulpraxis}

Viele Forschende im empirischen Bereich der Pädagogik legen Wert darauf, Wissenschaftler zu sein und interessieren sich kaum für schulpraktische Fragen. Sie forschen und publizieren und erwarten stillschweigend, dass die in der Bildungspolitik und im Schulwesen Tätigen ihre Veröffentlichungen lesen und in die Praxis umsetzen. Sie wählen dabei Darstellungsformen, die in der Praxis oft nicht verstanden und von den Lehrpersonen angesichts ihrer Belastungssituation im täglichen Unterricht auch kaum gelesen werden. Dazu kommt, dass sich viele Wissenschaftlerinnen und Wissenschaftler - abgesehen von einer Minderzahl von brillanten Forschenden - in bildungspolitischen Gremien für Praktiker zu wenig verständlich ausdrücken können, ihre Voten zwar meistens sorgfältig abwägen, es aber bei wissenschaftlich nicht eindeutig beantwortbaren Fragen nicht wagen, eine Empfehlung abzugeben, um ihren wissenschaftlichen Nimbus nicht zu verlieren. Oft vermeiden sie klare Aussagen auch, weil sie selbst erkennen, wie ihnen die Einsicht in die Alltagspraxis fehlt. Diese Feststellungen mögen hart klingen. Sie versuchen aber sichtbar zu machen, warum der Impact der empirischen Forschung trotz dem steigenden Einsatz von Ressourcen verhältnismässig gering ist. Um diese Schwäche zu überwinden, sollten die Forschenden die folgenden Aspekte beachten: 
(1) Sie müssen ihre Untersuchungen bildungsphilosophisch besser untermauern. $\mathrm{Zu}$ sehr entwickeln sich die maßgeblichen Forschungsvorhaben in Richtung von Output-Studien (Evaluationen), bei denen der kognitive Lernerfolg in Lernbereichen, die von der Gesellschaft als allein zukunftsträchtig betrachtet werden, vorherrscht, wobei das Messbare und nicht das ganzheitliche der Erziehung im Vordergrund steht. Dass dabei im Zusammenhang mit der Kompetenzforschung der Stellenwert der Wechselwirkungen zwischen deklarativem und prozeduralem Lernen weitgehend ungeklärt bleibt, ist für die künftige Gestaltung von Lehrplänen und Unterricht besonders kritisch (siehe dazu die Anmerkungen von Merkens 2011).

(2) Deshalb ist der Hypothesenformulierung viel mehr Beachtung zu schenken. Dazu sind bei jedem Forschungsprojekt alle Stakeholder miteinzubeziehen, damit die Hypothesen praxisgerechter sind (Design-BasedImplementation Research).

(3) Viel sorgfältiger sind bei allen Forschungsvorhaben die Rahmenbedingungen als intervenierende Variablen zu beachten. Vereinfachende experimentelle Studien in Laborsituationen mögen für die Gewinnung von Einsichten in grundlegende Abhängigkeiten und Wechselwirkungen verantwortlich sein (Beck 1981). Ihre einseitige Bevorzugung wäre aber Wissenschaft um der Wissenschaft willen. Experimente in künstlichen Umwelten sollten sich auf konkrete Fragestellungen in größeren Forschungsvorhaben beschränken. Um nur an einem Beispiel auf die Bedeutung der Rahmenbedingungen der Forschungsvorhaben hinzuweisen, sei das Thema der Belastungssituation von Lehrpersonen angesprochen: Viele Forschende sind bei Modellversuchen immer wieder überrascht, wenn die spätere alltägliche Umsetzung nicht zu den gleich guten Ergebnissen führt wie der Modellversuch. Dies ist nicht zuletzt darauf zurückzuführen, dass die alltägliche Belastungssituation der Lehrpersonen nicht als intervenierende Variable mit in die Untersuchung einbezogen wird, denn wenn die Lehrpersonen während dem Modellversuch alle Unterstützung erhalten, später aber allein gelassen werden, müssen sich andere Ergebnisse ergeben. Oder ganz allgemein ausgedrückt: Die Variablen der Schulrealität sind besser in empirische Untersuchungen einzubringen.

(4) Der Abschnitt „Diskussion“ in Forschungsberichten darf sich im Interesse des Impacts nicht nur auf die Hinweise zu weiteren Forschungsnotwendigkeiten beschränken, sondern es sollten auch Hinweise für die Implementation entworfen werden. Diese Hinweise müssen soweit als möglich eine Konsequenz aus den Forschungsergebnissen sein. Weil sie aber nie alles abdecken können, sind auch Hinweise auf die Grenzen der 
Implementation sowie auf offene Fragen notwendig, damit eine reflektierte Meinung der Forschenden zum Ausdruck kommt.

(5) Für den Impact der empirischen Forschung ist nicht nur die Implementationsforschung bedeutsam, sondern die Forschenden sollten sich auch konkret um die Umsetzung bemühen. Nachdem bekannt ist, dass Innovationen am Erfolgreichsten über Lehr- und Lernmaterialien eingeführt werden, sollten die Forschenden überall dort, wo es möglich ist, Lehrund Lernmaterialien zu ihrem Forschungsgegenstand entwickeln. Und in der Lehrerfortbildung sollten Mitglieder der Forschergruppe selbst Unterrichtseinheiten demonstrieren. Ein Beispiel möge zeigen, warum dieser praktische Aspekt bedeutsam ist. In einem Forschungsprojekt waren zur Validierung eines neuen Unterrichtsbeurteilungssystems fünf Forschende aus dem Bereich des Lehrerverhaltens eingeladen, Lektionen und Lehrerverhalten auf Video-Aufnahmen zu beurteilen. Nach vier Beurteilungen ergab sich, dass ein als streng geltender Beurteiler immer die besten Urteile abgab. Nach einer längeren Diskussion dieses Sachverhaltes resultierte eine Begründung: Der weniger streng Bewertende hat die Thematik selbst im Schulalltag unterrichtet und kannte deren Tücken der Unterrichtsgegenstände, was seine besseren Urteile erklärte. Dieses vielleicht banale - Beispiel will die Bedeutung des Einbezugs praktischer Aspekte in die empirische Forschung belegen.

\section{Der siebte Problemkreis: Der Umgang mit Forschungsberichten}

In seinem interessanten Beitrag zur Anwendung erziehungswissenschaftlichen Wissens gelangt Heid $(2011,503)$ zu einer bedenkenswerten Feststellung: Es sind die Praktiker und Politiker, die nach ihren eigenen Kriterien darüber entscheiden, ,was ihnen die jeweils beurteilten Forschungsergebnisse wert oder wofür sie ihnen nützlich sind. Die behauptete, vermeintliche oder tatsächliche Unbrauchbarkeit eines bestimmten Wissens, die jeweils erwünschte Praxis anzuleiten oder auch zu kritisieren, kann als ein (impliziertes) Beurteilungskriterium geltend gemacht und je nach sozialer Definitionsmacht praktisch, wissenschafts- und nicht zuletzt finanzpolitisch durchgesetzt werden“. Und später: Er will nicht „der Bildungspraxis den ,Dienst ${ }^{\star}$ der Bildungsforschung entziehen, sondern genau umgekehrt, die Qualität der Leistung erhöhen, die die Bildungsforschung für die Bildungspraxis eben nur unter den Bedingungen jener kompetenzfundierten $\mathrm{Zu}-$ ständigkeitsteilung zu erbringen vermag, die Bildungswissenschaften nicht 
ohne Gefährdung der Qualität ihrer eigenen Arbeit außer Kraft setzen können".

Die Forschenden müssen sich also intensiver um den Kontakt mit den Politikern, den Bildungsverantwortlichen und den Lehrkräften bemühen, um den Impact zu erhöhen. Dies gelingt aber erst, wenn diese Stakeholder die empirische Forschung verstehen wollen und verstehen. Machbar wird dies erst, wenn in der Lehrergrund- und Lehrerfortbildung der empirischen Forschung mehr Beachtung geschenkt wird, indem aber nicht primär Forschende ausgebildet werden, sondern die Studierenden in erster Linie lernen, Forschungsergebnisse $\mathrm{zu}$ analysieren und $\mathrm{zu}$ interpretieren sowie ihre Relevanz für die Bildungspraxis zu beurteilen (Möglichkeiten der Forschung und der Anwendungsmöglichkeiten mit ihren Grenzen in der Praxis bewerten). Dazu sind das Forschende Lernen und Praktische Übungen im Schulalltag schon im Lehrerstudium in didaktischer Weise geschickt zu kombinieren. Aber auch das administrative Personal im Bildungsbereich ist in erziehungswissenschaftlichen Fragen stets weiterzubilden, denn viele gute Erkenntnisse aus der empirischen erziehungswissenschaftlichen Forschung werden infolge verwaltungstechnischer Hürden nicht umgesetzt, sei es, weil die Bildungsverwaltung keinen Bezug zur Mehrebenenbetrachtung hat, mit Forschungsergebnissen nicht umzugehen versteht, politische und rechtliche Schranken bestehen, oder sei es, weil die Bildungsverwaltung Machtverluste befürchten. Am Beispiel der Forschungen zur selbständigen Schule (Dubs 2011) lässt sich dies deutlich erkennen: Studien zur Frage des Schulleitungshandelns bringen zunehmend widersprüchliche Ergebnisse, weil die Rahmenbedingungen von Land zu Land sehr verschieden sind und in vielen Ministerien die Entwicklung zur Dezentralisierung der Schule mangels Kenntnissen aus der empirischen erziehungswissenschaftlichen Forschung nicht bekannt sind.

\section{Nachwort}

Die empirische Forschung wird trotz allen Problemen für die Entwicklung sehr bedeutsam bleiben (siehe die Entwicklung bei Jäger et al. 2010). Dies, weil sich leider auch im Bildungswesen immer häufiger Polarisierungstendenzen zwischen verschiedensten Gruppierungen mit immer stärkerem dogmatischem Charakter ergeben. Seit - besonders in der Schweiz - die politischen Parteien das Bildungswesen in Wahlkämpfen $\mathrm{zu}$ einem der Profilierungsinstrumente entwickeln, wird das kurzfristige und modische pädagogische Denken zum permanenten Umgang mit Slogans führen und Pendelschläge eher verstärken. Deshalb werden klärende Erkenntnisse aus der empirischen Forschung immer wichtiger. Einen echten Beitrag mit einer 
nachhaltigen Wirkung, also ein Impact, wird sie aber nur erhalten, wenn sie sich in Richtung eines Design-Based-Implementation Research entwickelt, sie Aussagen zu maßgeblichen Problemen zu vermitteln versteht, die atomistische Tendenz zu kleinen, die Rahmenbedingungen ungenügend berücksichtigenden Forschungsvorhaben verlässt, wirkungsorientiert arbeitet, systematisch mit allen Stakeholdern zusammenwirkt und bezüglich der Implementationsmöglichkeiten immer wieder selbstkritisch fragt: So what?

\section{Literatur}

Beck, K. (1981): Die semantische Struktur mehrdimensionaler Ratings als Methodenproblem der Unterrichtsforschung. In: Zeitschrift für Empirische Pädagogik, S. $65-82$.

Dubs, R. (2006): Bildungsstandards und kompetenzorientiertes Lernen. In: G. Minnameier/ E. Wuttke (Hrsg.). Berufs- und wirtschaftspädagogische Grundlagenforschung. Lehr-Lern-Prozesse und Kompetenzdiagnostik. Frankfurt am Main: Lang, S. 161-175.

Dubs, R. (2009): Lehrerverhalten. Ein Beitrag zur Interaktion von Lehrenden und Lernenden im Unterricht. Steiner: Stuttgart.

Dubs, R. (2011): Die teilautonome Schule. Ein Beitrag zu ihrer Gestaltung aus politischer, rechtlicher und schulischer Sicht. Berlin: edition sigma.

Heid, H. (2011): Die Bedingungen der Anwendung erziehungswissenschaftlichen Wissens. In: O. Zlatkin-Troitschanskaia (Hrsg.). Stationen Empirischer Bildungsforschung. Traditionslinien und Perspektiven. Wiesbaden: VS Verlag für Sozialwissenschaften, S. 490-508.

Jäger, R. S./Nenninger, P./Pettilon, H./Schwarz, B./Wolf, B. (Hrsg.) (2010): Empirische Pädagogik 1990-2010. Eine Bestandesaufnahme der Forschung in der Bundesrepublik Deutschland. Landau: Verlag Empirische Pädagogik.

Komisar, B. P./McCellan, J. E. (1968): In: B. O. Smith \& R. Ennis (Eds.). Language and Concepts in Education. Chicago: Rand McNally, S. 221-237.

Merkens, H. (2011): Zukunft der Bildungsforschung. In: O. Zlatkin-Troitschanskaia (Hrsg.). Stationen Empirischer Bildungsforschung. Traditionslinien und Perspektiven. Wiesbaden: VS Verlag für Sozialwissenschaften, S. 509-519.

Penuel, W. R./Fishman, B. J./Haugan Cueng, B./Sabelli, N. (2011): Organizing Research and Development at the Intersection of Learning, Implemantation and Design. In: Educational Researcher, 40(7), S. 331-337.

Stamm, M. (2003): Evaluation und ihre Folgen für die Bildung. Eine unterschätzte pädagogische Herausforderung. Münster: Waxmann. 



\section{Betriebliche Weiterbildung als interdisziplinäres Forschungsfeld - Annäherung an eine berufs- und wirtschaftspädagogische Perspektive}

Uwe Elsholz

\section{Ausgangslage}

In der Berufs- und Wirtschaftspädagogik wird seit geraumer Zeit die zu geringe Bedeutung der beruflich-betrieblichen Weiterbildung im Rahmen des disziplinären Diskurses moniert (vgl. u.a. Arnold/Gonon 2006, S. 185; Diettrich/Vonken 2009; Meyer/Elsholz 2009). Trotz zunehmender praktischer Relevanz für den Einzelnen angesichts wechselnder beruflicher Anforderungen und der Durchsetzung des Konzepts lebenslangen Lernens, wird in diesem Feld einhellig ein Theoriedefizit konstatiert.

Dieser Beitrag ist darauf angelegt, der angedeuteten defizitären Bearbeitung beruflich-betrieblicher Weiterbildung durch die Berufs- und Wirtschaftspädagogik entgegenzuwirken. ${ }^{1}$ Angesichts einer unübersichtlichen Ausgangslage erscheint zunächst eine (Selbst-)verständigung darüber notwendig, wodurch sich der berufs- und wirtschaftspädagogische Zugang im „disziplinär umkämpften Feld“ (Meyer/Elsholz 2009, S. 4) der betrieblichen Weiterbildung auszeichnet.

Einführend erfolgt eine kurze Zustandsbeschreibung hinsichtlich der Behandlung der beruflich-betrieblichen Weiterbildung durch die Berufs- und Wirtschaftspädagogik. Um eine genuin berufs- und wirtschaftspädagogische Perspektive auf das Gegenstandsfeld zu entwickeln, wird daran anknüpfend zunächst analysiert, welche spezifischen Schwerpunkte bei der Betrachtung betrieblicher Weiterbildung durch verwandte wissenschaftliche Disziplinen vorherrschen. Dazu werden die Perspektiven der Erwachsenenpädagogik, der Arbeits- und Organisationspsychologie, der Industriesoziologie, der Betriebssowie der Volkswirtschaftslehre herausgearbeitet.

Vor diesem Hintergrund erfolgt im vierten Abschnitt eine Annäherung an eine originäre Perspektive der Berufs- und Wirtschaftspädagogik auf den Gegenstandsbereich. Ausgangspunkt sind hier neuere Handbücher sowie Veröffentlichungen zum Basiscurriculum Berufs- und Wirtschaftspädagogik

1 Dieses Positionspapier beruht auf der Vorbereitung und Durchführung von drei aufeinander aufbauenden Foren zur beruflich-betrieblichen Weiterbildung im Rahmen von Tagungen der Sektion Berufs- und Wirtschaftspädagogik in den Jahren 2009-2011. 
(BWP 2002; Dehnbostel 2010). Auf Grundlage dieser kontrastierenden Betrachtung wird ein Vorschlag zur Beschreibung einer genuin berufs- und wirtschaftspädagogischen Perspektive hinsichtlich der Bearbeitung des Forschungsfeldes betriebliche Weiterbildung unterbreitet. Abschließend werden in einem Ausblick zukünftige disziplinäre Herausforderungen und Fragestellungen aufgeführt.

\section{Betriebliche Weiterbildung als (marginalisierter) Gegenstand der Berufs- und Wirtschaftspädagogik}

Betriebliche Weiterbildung wird gegenwärtig als randständiges Thema der Disziplin Berufs- und Wirtschaftspädagogik behandelt (vgl. Meyer/Elsholz 2009; Diettrich/Vonken 2009). In Forschung und Lehre ist eine Fokussierung auf die berufliche Erstausbildung, schulische Lehr- und Lernprozesse und die Lehrerbildung zu konstatieren. Berufliches und betriebliches Lernen und sowie beruflich-betriebliche Weiterbildung sind in den Forschungsarbeiten der Disziplin weniger sichtbar. Verschiedene Indizien stützen diese Ausgangsthese:

- Eine Analyse über 25 Jahrgänge der Zeitschrift für Berufs- und Wirtschaftspädagogik (1979-2004) hat gezeigt, dass nur eine geringe wissenschaftliche Ausdifferenzierung der betrieblichen Weiterbildungsforschung zu verzeichnen ist (vgl. Meyer/Neumann 2005).

- Die Tagungsprogramme sowie die anschließenden Publikationen der Sektionstagungen aus den Jahren 2005-2010 weisen beruflichbetriebliche Weiterbildung nur punktuell als eigenes thematisches Feld aus. Ebenso ist das Fehlen einer gesonderten Arbeitsgruppe oder einer dauerhaften thematisch einschlägigen Kooperation innerhalb der Sektion zu konstatieren.

- In inhaltlicher Hinsicht wird wiederholt ein Theoriedefizit betrieblicher Weiterbildung beklagt (vgl. u.a. Elsholz/Molzberger 2007, S. 161). Exemplarisch stellen Rebmann und Tenfelde ein Auseinanderfallen von Theorie und Praxis fest: „Zwischen einer durchaus innovativen Praxis der Beförderung betrieblichen Lernens einerseits und der theoriegeleiteten Re-Interpretation vorfindlicher Praxis andererseits klafft eine große Lücke. Die Praxis betrieblichen Lernens ist nur in Fragmenten auf theoretisch wie empirisch gehaltvolle Referenztheorien $\mathrm{zu}$ beziehen. Möglicherweise tragfähige Referenztheorien erreichen in ihrer abstrakten Form (noch) nicht die Praxis“. (Rebmann/Tenfelde 2008, S. V) 
Vor diesem als defizitär beschriebenen Hintergrund werden im Folgenden zunächst die Perspektiven benachbarter Disziplinen herausgearbeitet, um sich anschließend in einer Kontrastierung einer originär berufs- und wirtschaftspädagogischen Sichtweise anzunähern. Dabei wird - aus Gründen der Handhabbarkeit - zunächst nur auf den Teilbereich der betrieblichen Weiterbildung fokussiert und die berufliche Weiterbildung in diesem Beitrag vernachlässigt. Diese Unterscheidung ist insofern von Bedeutung, als dass beide Felder unterschiedlichen Handlungslogiken folgen, wie Harney (1998) herausgearbeitet hat. Während bei der betrieblichen Weiterbildung der Organisationsbezug im Vordergrund steht und die Stärkung der professionellen Autonomie der Weiterbildungsteilnehmer eher ein spannungsgeladener Nebeneffekt ist, verhält sich dies bei der beruflichen Weiterbildung nahezu umgedreht. Hier steht die berufliche Entwicklung des Weiterbildungsteilnehmers im Zentrum, eine betriebliche Verwertbarkeit bleibt jedoch eine notwendige Nebenbedingung für die langfristige Legitimation der beruflichen Weiterbildung (vgl. ebd., S. 254f.).

\section{Die Perspektiven benachbarter Wissenschaftsdisziplinen auf betriebliche Weiterbildung}

Betriebliche Weiterbildung ist Forschungsgegenstand unterschiedlicher wissenschaftlicher (Teil-)disziplinen. Um einen genuinen Zugriff berufs- und wirtschaftspädagogischer Betrachtung in Abgrenzung zu anderen Sichtweisen herauszuarbeiten, werden nachfolgend zunächst die prioritären Perspektiven benachbarter Disziplinen dargestellt. Dabei wird vorrangig auf Handbücher und Standardwerke der jeweiligen Wissenschaftsdisziplin rekurriert, da darin das jeweils zu Grunde liegende Selbstverständnis zum Ausdruck kommt. Sofern dieser Zugang keine ausreichenden Informationen für die Frage der disziplinären Betrachtung betrieblicher Weiterbildung geliefert hat, wurden zusätzlich andere exemplarische Publikationen herangezogen. Dass es sich bei der nachfolgenden Darstellung dennoch um eine verkürzte Zuspitzung handelt, ist der intendierten starken Kontrastierung der disziplinären Sichtweisen auf die betriebliche Weiterbildung geschuldet.

Die Abbildung verdeutlicht die beschriebene Zugangsweise, die versucht, den Gegenstandsbereich „Betriebliche Weiterbildung“ je unter einer disziplinären Perspektive zu erfassen. 


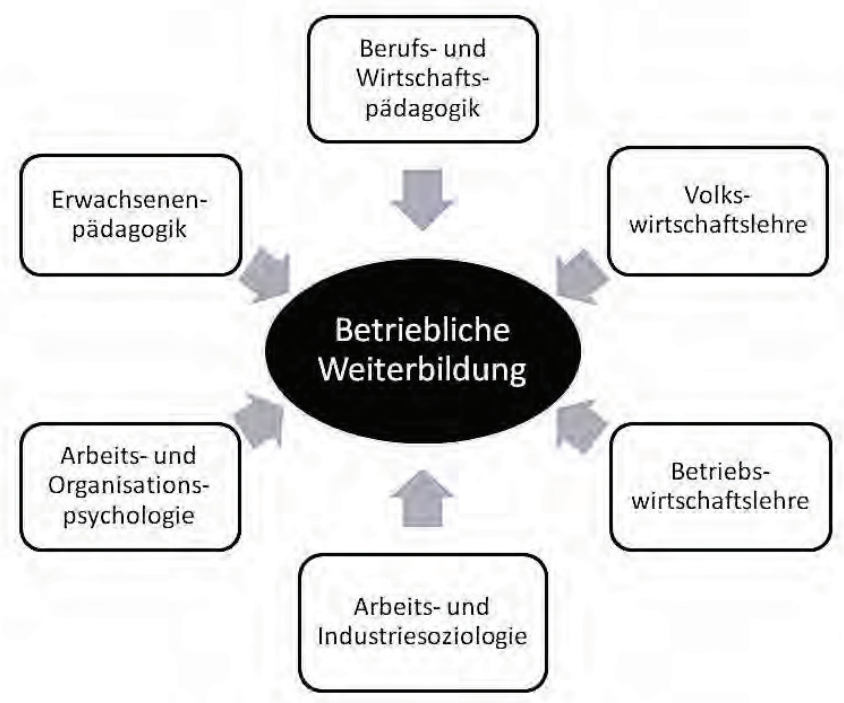

Eine volkswirtschaftliche Perspektive betrachtet betriebliche Weiterbildung in erster Linie unter arbeitsmarktpolitischen Gesichtspunkten. Die Volkswirtschaftslehre untersucht beispielsweise konjunkturelle Auswirkungen auf die Verbreitung von Weiterbildung in den Betrieben oder fragt längerfristige Trends hinsichtlich der $\mathrm{Zu}$ - und Abnahme von Weiterbildungsangeboten ab. Dies geschieht ausdifferenziert z.T. in Abhängigkeit von der Betriebsgröße, der Region oder der Branche, mit Blick auf unterschiedliche Zielgruppen der Weiterbildung oder in Bezug auf andere Determinanten wie Alter, Geschlecht oder Migrationshintergrund. Eine volkswirtschaftliche Betrachtung nimmt dementsprechend vor allem quantitative Untersuchungen zur Verbreitung von Angeboten an betrieblicher Weiterbildung vor. Exemplarisch hierfür steht vor allem das Betriebspanel des Instituts für Arbeitsmarkt- und Berufsforschung (vgl. u.a. Leber 2009; Bellmann/Leber 2010).

Unter betriebswirtschaftlichen Gesichtspunkten wird betriebliche Weiterbildung in der wissenschaftlichen Diskussion vorrangig als Teil personalwirtschaftlichen Handelns und insbesondere der Personalentwicklung betrachtet (vgl. u.a. Olfert 2010, S. 385ff.). In diesem Zusammenhang hat die betriebliche Weiterbildung einen Beitrag zum ökonomischen Unternehmenserfolg zu leisten. Es werden in erster Linie Aspekte der Bedarfsermittlung, der Kostenstrukturen und des Weiterbildungscontrollings behandelt. In der 
Regel wird eine Quantifizierung der In- und Outputbedingungen betrieblicher Weiterbildung angestrebt (vgl. ebd.).

Die Arbeits- und Industriesoziologie thematisiert Weiterbildung vorrangig im Zusammenhang mit veränderten betrieblichen Produktionskonzepten und daraus folgenden Qualifikationsanforderungen an die Beschäftigten. Exemplarisch stehen dafür einige Klassiker der Industriesoziologie wie die Untersuchung von Kern und Schumann: „Das Ende der Arbeitsteilung?“ (1984) oder der „Trendreport Rationalisierung“ von Schumann u.a. (1994). Diese Bände zeigen auf, wie sich veränderte Produktionskonzepte in unterschiedlichen Branchen in erweiterten Arbeitsaufgaben für die Beschäftigten niederschlagen und sich daraus neue Qualifikationsanforderungen ergeben. In ähnlicher Weise zeichnen auch Baethge/Baethge-Kinsky (2006) im Handbuch der Berufsbildung aus industriesoziologischer Perspektive den Wandel von einer fordistischen zu einer postfordistischen und damit prozessorientierten Arbeitsorganisation nach. Sie beschreiben die Folgen dieser Veränderungsprozesse mit Blick auf die Qualifikationsanforderungen an die Beschäftigten. Es ist allerdings zu konstatieren, dass im letzten Jahrzehnt die Frage veränderter und erweiterter Qualifikationsanforderungen in der industriesoziologischen Debatte einen geringeren Stellenwert eingenommen hat als dies in den 1980er und 1990er Jahren der Fall war. Zu vermuten ist, dass dies damit zu tun hat, dass in der letzten Dekade eher Tendenzen zur (zumindest partiellen) Re-Taylorisierung in den Betrieben zu verzeichnen waren (vgl. u.a. Kuhlmann 2009).

Die Arbeits- und Organisationspsychologie thematisiert Fragen betrieblicher Weiterbildung vorrangig im Zusammenhang mit der Qualifizierung für und in der Arbeitstätigkeit (vgl. Ulich 2005; Sonntag/Stegmaier 2007). Für die Arbeitswissenschaft als eng verwandte Disziplin trifft dies in ähnlicher Weise zu (vgl. Schlick u.a. 2010, S. 72). Es wird in dieser Sichtweise damit immer ,vom Arbeitsplatz her" gedacht und dessen Gestaltung in den Blick genommen. Weiterbildung und Qualifizierungsmöglichkeiten sind Thema im Rahmen prospektiver Arbeitsgestaltung und werden hier unverzichtbarer Bestandteil.

In der Erwachsenenpädagogik - als eine der Berufs- und Wirtschaftspädagogik verwandte erziehungswissenschaftliche Teildisziplin - wird betriebliche Weiterbildung vorrangig aus einer individuellen Aneignungsperspektive thematisiert. Exemplarisch stehen dafür zwei Habilitationen von Ludwig (2000) und Allespach (2004), die sich mit betrieblicher Weiterbildung beschäftigen und sich beide auf die Lerntheorie Holzkamps als theoretischer Basis beziehen. Damit wird ein spezifischer subjektwissenschaftlicher Lernbegriff zu Grunde gelegt und eine individuelle Perspektive auf die Weiterbildung präferiert.

Vor dem Hintergrund der hier skizzierten primären Fragestellungen anderer wissenschaftlicher Disziplinen soll nun herausgearbeitet werden, was 
eine genuin berufs- und wirtschaftspädagogische Perspektive auf betriebliche Weiterbildung auszeichnet.

\section{Annäherung an eine berufs- und wirtschaftspädagogische Perspektive auf betriebliche Weiterbildung}

Ein Blick in aktuelle Handbücher und Lexika der Berufs- und Wirtschaftspädagogik zeichnet zunächst ein ernüchterndes Bild: Sowohl das Handbuch Berufs- und Wirtschaftspädagogik (Nickolaus u.a. 2010) als auch der Einführungsband „Berufs- und Wirtschaftspädagogik. Eine Einführung in Strukturbegriffe" (Rebmann u.a. 2011) thematisieren lediglich die berufliche Weiterbildung - die betriebliche Weiterbildung wird nur implizit behandelt. Zudem wird auch die berufliche Weiterbildung fast ausschließlich unter organisatorisch-strukturellen und nicht unter pädagogischen Aspekten betrachtet. Fragen nach Lernprozessen auf der Mikroebene oder nach der curricularen Gestaltung auf einer Mesoebene werden nicht ausgearbeitet. Das Handbuch Berufs- und Wirtschaftspädagogik - das sich am Basiscurriculum Berufs- und Wirtschaftspädagogik orientiert - behandelt die betriebliche Weiterbildung lediglich im Rahmen eines Beitrags zum Lernen im Prozess der Arbeit (vgl. Dehnbostel u.a. 2010).

Als zielführender erweisen sich allerdings zwei weitere Quellen, die dabei helfen, eine genuin berufs- und wirtschaftspädagogische Perspektive zu beschreiben:

Das Handbuch Berufsbildung (Arnold/Lipsmeier 2006) enthält einen Aufsatz von Rolf Arnold unter dem Titel „Neue Methoden betrieblicher Bildungsarbeit". Zudem liegt in der Schriftenreihe zum Basiscurriculum Berufsund Wirtschaftspädagogik ein Studientext unter dem Titel „Betriebliche Bildungsarbeit. Kompetenzbasierte Aus- und Weiterbildung im Betrieb" von Dehnbostel (2010) vor. In diesem Text, der damit als paradigmatisch für eine disziplinäre Betrachtung angesehen werden kann, werden folgende Aspekte behandelt:

- Betriebliche Bildungsarbeit

- Modelle und Konzepte arbeitsbezogenen Lernens

- Betriebliche Lernarten

- Konzepte beruflich-betrieblichen Lernens

- Betriebliche Lernorte und Lernformen 
- Lernförderliche Arbeitsgestaltung

- Begleitung und Beratung

- Kompetenzanalysen

- Anerkennung informell erworbener beruflicher Kompetenzen

Nimmt man den genannten Beitrag von Arnold (2006) dazu und betrachtet die genannten Inhalte vor dem Hintergrund der Zugänge anderer Disziplinen, so wird hier vorgeschlagen, als den wesentlichen Kern einer berufs- und wirtschaftspädagogischen Perspektive auf die betriebliche Weiterbildung folgenden Terminus aufzufassen:

Der genuine Fokus einer berufs- und wirtschaftspädagogischen Perspektive auf betriebliche Weiterbildung liegt in der Untersuchung und Gestaltung betrieblicher Maßnahmen zur Kompetenzentwicklung.

Eine solche Perspektive grenzt sich von den dargestellten Zugängen anderer Disziplinen eindeutig ab, denn diese betrachten entweder prioritär die individuelle Aneignungsperspektive (vor allem die Erwachsenenbildung) oder den strukturellen Beitrag der Weiterbildung für die Erfüllung von Arbeitsaufgaben, zum Unternehmenserfolg oder zur gesamtwirtschaftlichen Entwicklung.

Unter diesem Terminus „Untersuchung und Gestaltung betrieblicher Maßnahmen zur Kompetenzentwicklung“ kann auch ein Großteil weiterer Publikationen zur betrieblichen Weiterbildung verortet werden, die aus berufs- und wirtschaftspädagogischer Perspektive verfasst sind:

- Mit Blick auf neuere Qualifizierungsarbeiten gilt dies insbesondere für die Publikationen von Molzberger (2007), die sich mit Rahmungen informeller Lernprozesse in der IT-Branche befasst sowie von Schröder (2009), der sich mit Arbeits- und Lernaufgaben als Bestandteil der ITWeiterbildung beschäftigt.

- Eine Vielzahl der jüngeren Publikationen zu diesem Feld in der Zeitschrift für Berufs- und Wirtschaftspädagogik (z.B. Bohlinger/ Heidecke 2009; Kohl/Molzberger 2005) sowie der BWP - Berufsbildung in Wissenschaft und Praxis (z.B. Elsholz 2011; Behringer/Käpplinger 2011; Baumgartner/Seifried 2011) sind hier ebenso zu subsumieren.

- Das Gleiche gilt für die Aktivitäten im Rahmen der CVTS-Erhebung, die auf die Untersuchung betrieblicher Maßnahmen zur Kompetenzentwicklung zielen (vgl. Behringer u.a. 2009).

Der hier unterbreitete Vorschlag hinsichtlich dient als Zuspitzung der Abgrenzung und damit Sichtbarmachung eines spezifischen berufs- und wirtschaftspädagogischen Zugangs. Dies bedeutet jedoch nicht, dass andere Arbeiten - etwa historische Zugänge (z.B. Büchter 2010) oder Fragen der 
Professionalisierung des Bildungspersonals - nicht ebenfalls wichtige Bestandteile berufs- und wirtschaftspädagogischer Forschung darstellen.

\section{Ausblick}

In diesem Beitrag wurde empfohlen, zur Schärfung einer berufs- und wirtschaftspädagogischen Perspektive auf betriebliche Weiterbildung die Untersuchung und Gestaltung betrieblicher Maßnahmen zur Kompetenzentwicklung als originäre disziplinäre Fragestellung aufzufassen. Neben der Diskussion, ob die vorgeschlagene Fokussierung eine angemessene Beschreibung darstellt, zeigen sich im Zusammenhang mit der weiteren Bearbeitung der ausgeführten Defizite hinsichtlich der Sichtbarkeit und Theorieentwicklung beruflich-betrieblicher Weiterbildung weitere Herausforderungen. Für die disziplinäre berufs- und wirtschaftspädagogische Diskussion stellen sich dabei folgende Fragen:

- Welche relevanten Theorieansätze gibt es im Feld beruflich-betrieblicher Weiterbildung und welche müssten entwickelt werden?

- Welches sind die angemessenen Schlüsselbegriffe der Berufs- und Wirtschaftspädagogik zur Beschreibung der betrieblichen Weiterbildung?

- Welche Bedeutung haben „Arbeit“ und „Beruf“ als Bezugsgrößen berufspädagogischer Theoriebildung im Hinblick auf Theorieansätze für die betriebliche Weiterbildung?

Schließlich geht es auch um die Frage, wo die Grenzen eines berufs- und wirtschaftspädagogischen Zugriffs liegen und bei welchen Themenfeldern mit Nachbardisziplinen kooperiert werden sollte. Exemplarisch stellt sich diese Herausforderung etwa bei der Gestaltung lernförderlicher Arbeit. Hier kann ein berufs- und wirtschaftspädagogischer Ansatz durch die Entwicklung arbeitsintegrierter Lernformen (vgl. u.a. Elsholz/Molzberger 2007) einen wichtigen Beitrag für die Ausgestaltung betrieblicher Maßnahmen leisten. Allerdings ist es mit Blick auf die Veränderung konkreter Arbeitstätigkeiten und der betrieblichen Arbeitsgestaltung notwendig, arbeitswissenschaftliche Ansätze ebenfalls hinzuzuziehen. In ähnlicher Weise lässt sich die Notwendigkeit eines interdisziplinären Zugriffs auch für das Thema Demografie beschreiben. Auch hier kann die Berufs- und Wirtschaftspädagogik durch die Entwicklung und Erprobung adäquater Lernformen einen wichtigen Beitrag leisten, bleibt aber auf das Zusammenwirken mit betriebswirtschaftlich und arbeitspsychologisch fundierten Konzepten zur alters- und alternsgerechten Personalentwicklung angewiesen. 


\section{Literatur}

Allespach, M. (2005): Betriebliche Weiterbildung als Beteiligungsprozess. Subjektive Bedeutsamkeiten als Grundlage für eine partizipative Bildungsplanung. Marburg

Arnold, R. (2006): Neue Methoden betrieblicher Bildungsarbeit. In: Arnold, R./ Lipsmeier, A. (Hrsg.): Handbuch der Berufsbildung. 2. Aufl., Wiesbaden, S. 355369.

Arnold, R./Gonon, P. (2006): Einführung in die Berufspädagogik. Opladen, Bloomfield Hills.

Baethge, M./Baethge-Kinsky, V. (2006): Ökonomie, Technik, Organisation. Zur Entwicklung von Qualifikationsstruktur und qualitativem Arbeitsvermögen. In: Arnold, R./Lipsmeier, A. (Hrsg.): Handbuch der Berufsbildung. 2. Aufl., Wiesbaden, S. 153-173.

Baumgartner, A./Seifried, J. (2011): Umgang mit Fehlern am Arbeitsplatz. Das Beispiel Gastronomie. In: BWP - Berufsbildung in Wissenschaft und Praxis 40, $\mathrm{H}$. 1, S. 20-24.

Behringer, F./Käpplinger, B. (2011): Arbeitsplatznahe Lernformen und Lernortvielfalt in der betrieblichen Weiterbildung. Wachsende Bedeutung in ganz Europa oder deutsches Spezifikum? In: BWP - Berufsbildung in Wissenschaft und Praxis 40, H. 1, S. 15-19.

Behringer, F./Käpplinger, B./Pätzold, G. (Hrsg.) (2009): Betriebliche Weiterbildung. Der Continuing Vocational Training Survey (CVTS) im Spiegel nationaler und europäischer Perspektiven, Stuttgart.

Bellmann, L./Leber, U. (2010): Betriebliche Weiterbildung: In der Krise bleibt das Bild zwiespältig. In: IAB-Forum, Nr. 1, S. 16-19.

Bohlinger, S./Heidecke, L. (2009): Pluralisierung von Lernorten und Lernformen in der betrieblichen Weiterbildung. In: ZBW - Zeitschrift für Berufs- und Wirtschaftspädagogik 105, H. 3, S. 452-459.

Büchter, K. (2010): Weiterbildung für den Arbeitsmarkt und im Betrieb 1919-1933: Hintergründe, Kontexte, Formen und Funktionen. Hamburg.

BWP (2002): Basiscurriculum Berufs- und Wirtschaftspädagogik. Hrsg. von der Sektion Berufs- und Wirtschaftspädagogik. Online: http://www.bwpdgfe.de/sektion/positionen/curriculum/ (10.03.2011).

Dehnbostel, P. (2010): Betriebliche Bildungsarbeit. Kompetenzbasierte Aus- und Weiterbildung im Betrieb. Baltmannsweiler.

Dehnbostel, P./Fürstenau, B./Klusmeyer, J./Rebmann, K. (2010): Kontextbedingungen beruflichen Lernens: Lernen in der Schule und im Prozess der Arbeit. In: Nickolaus, R./Pätzold, G./Reinisch, H./Tramm, T. (Hrsg.): Handbuch Berufsund Wirtschaftspädagogik. Bad Heilbrunn, S. 87-98.

Diettrich, A./Vonken, M. (2009): Zum Stellenwert der betrieblichen Aus- und Weiterbildung in der Berufs- und Wirtschaftspädagogik. In: bwp@ Berufs- und Wirtschaftspädagogik-online, Ausgabe 16, 1-20. Online: http:/www.bwpat.de/ ausgabe16/diettrich_vonken_bwpat16.pdf (30-06-2009).

Elsholz, U. (2011): Systematisierung und Dokumentation arbeitsintegrierter Weiterbildung. Ein softwaregestütztes Beispiel aus der Abfallverbrennung. In: BWP Berufsbildung in Wissenschaft und Praxis 40, H. 1, S. 32-35. 
Elsholz, U./Molzberger, G. (2007): Zur Erschließung betrieblichen Lernens in der Arbeit - theoretische, empirische und praktische Aufgaben. In: Münk, D./van Buer, J./Breuer, K./Deißinger, T. (Hrsg.): Hundert Jahre kaufmännische Ausbildung in Berlin. Opladen, Farmington Hills, S. 154-163.

Harney, K. (1998): Handlungslogik betrieblicher Weiterbildung. Stuttgart

Kern, H./Schumann, M. (1984): Das Ende der Arbeitsteilung? : Rationalisierung in der industriellen Produktion. München.

Kohl, M./Molzberger, G. (2005): Lernen im Prozess der Arbeit - Überlegungen zur Systematisierung betrieblicher Lernformen in der Aus- und Weiterbildung. In: ZBW - Zeitschrift für Berufs- und Wirtschaftspädagogik 101, H. 3, S. 349-363

Kuhlmann, M. (2009): Perspektiven der Arbeitspolitik nach der Krise. Entwicklungslinien und Handlungsbedingungen. In: WSI-Mitteilungen, Jg. 62, H. 12, S. 675682.

Leber, U. (2009): Betriebsgröße, Qualifikationsstruktur und Weiterbildungsbeteiligung. Ergebnisse aus dem IAB-Betriebspanel. In: Behringer, F.; Käpplinger, B.; Pätzold, G. (Hrsg.) (2009): Betriebliche Weiterbildung. Der Continuing Vocational Training Survey (CVTS) im Spiegel nationaler und europäischer Perspektiven. Stuttgart, S. 149-168.

Ludwig, J. (2000): Lernende verstehen. Lern- und Bildungschancen in betrieblichen Modernisierungsprojekten. Bielefeld.

Meyer, R./Neumann, G. (2005): Zwischen wissenschaftlicher Marginalisierung und praktischer Relevanz - Betriebliche Weiterbildung im Diskurs der Berufs- und Wirtschaftspädagogik. In: Elsholz u.a. (Hrsg.): Berufsbildung heißt: Arbeiten und Lernen verbinden. Münster/New York, S. 213-226.

Meyer, R./Elsholz, U. (2009): Berufliche und betriebliche Weiterbildung als Gegenstand der Berufs- und Wirtschaftspädagogik - Desiderata und neue Perspektiven für Theorie und Forschung. In: bwp@ Berufs- und Wirtschaftspädagogik - online, Ausgabe 16, 1-15. Online: http://www.bwpat.de/ ausgabe16/ meyer_elsholz bwpat16.pdf (30-06-2009).

Molz̄berger, G. (2007): Rahmungen informellen Lernens. Zur Erschließung neuer Lern- und Weiterbildungsperspektiven. Wiesbaden.

Nickolaus, R./Pätzold, G./Reinisch, H./Tramm, T. (Hrsg.) (2010): Handbuch Berufsund Wirtschaftspädagogik. Bad Heilbrunn.

Olfert, K. (2010): Personalwirtschaft. 14., verbesserte und aktualisierte Aufl., Herne

Rebmann, K./Tenfelde, W. (2008): Betriebliches Lernen. Explorationen zur theoriegeleiteten Begründung, Modellierung und praktischen Gestaltung arbeitsbezogenen Lernens. München.

Rebmann, K./ Tenfelde, W./Schlömer, T. (2011): Berufs- und Wirtschaftspädagogik. Eine Einführung in Strukturbegriffe. 4. Aufl., Wiesbaden.

Schlick, C./Bruder, R./Luczak, H. (2010): Arbeitswissenschaft. 3., vollst. überarb. und erw. Aufl., Berlin.

Schröder, T. (2009): Arbeits- und Lernaufgaben für die Weiterbildung. Bielefeld.

Schumann, M./Baethge-Kinsky, V./Kuhlmann, M. (1994): Trendreport Rationalisierung. Automobilindustrie, Werkzeugmaschinenbau, chemische Industrie. Berlin.

Sonntag, K./Stegmaier, R. (2007): Arbeitsorientiertes Lernen. Zur Psychologie der Integration von Lernen und Arbeit. Stuttgart.

Ulich, E. (2005): Arbeitspsychologie, 6. Aufl., Stuttgart. 


\section{Reflexive Wirtschaftspädagogik: Wie Ethik, Neo-Institutionalismus und Europädagogik neue Perspektiven eröffnen könnten}

\section{Georg Tafner}

Dieser Beitrag beschreibt die Arbeitsskizze für die sich in Arbeit befindliche Habilitationsschrift zum Thema reflexive Wirtschaftspädagogik. Ausgehend von der Erkenntnis, dass Ökonomie und Pädagogik kulturelle Phänomene sind (Kapitel 1), kann Ethik nicht, einem vermeintlichen Naturgesetz gleich, auf eine rein ökonomische Ethik reduziert werden (Kapitel 2). Vielmehr leiten Institutionen wie Recht, Moral oder Selbstverständlichkeiten das Denken, nehmen aber weder die Freiheit noch die Verantwortung (Kapitel 3). In der europäischen Dimension vermischen sich die Institutionen zu einem fast undurchdringlichen Gemenge, sodass die Europäische Union als Teil Europas zwar rechtlich definiert, aber als Metapher für viele Interpretationen offen bleibt (Kapitel 4). Europapädagogik zielt darauf ab, die europäische Supranationalität in ihrer sozio-ökonomischen Dimension begreifbar zu machen.

Ethik, Neo-Institutionalismus und Europapädagogik können neue Perspektiven eröffnen und zu einer Neu-Akzentuierung der Wirtschaftspädagogik führen (Kapitel 5).

\section{1. Ökonomie und Pädagogik sind kulturelle Phänomene}

Mit den nicht unproblematischen Bezeichnungen (vgl. Lisop 2009, 1-18) „Employability“ und „Kompetenzentwicklung“ werden in der aktuellen berufs- und wirtschaftspädagogischen Literatur wesentliche Ziele der Wirtschaftspädagogik beschrieben (vgl. Thoma 2011, 15) - wirtschaftliche Erziehung als solche wird weniger thematisiert. Dies könnte drei Ursachen haben: Erstens wird dem Sachverhalt der Erziehung ausgewichen (vgl. Prange 2000, 7). Zweitens wird ökonomisches Denken und Handeln als wertfrei (vgl. Wöhe \& Döring 2010, 1) oder drittens als Ethik selbst (vgl. Homann 2008, 199-201) verstanden. Dahinter könnte eine vermeintliche NichtNormativität stehen, die andere Sichtweisen als normativ zurückweist.

Der Inhalt der Wirtschaftspädagogik fokussiert vor allem auf die Wirtschaftsdidaktik, die Förderung von beruflichen Kompetenzen, der Professionalisierung der Bildungskräfte sowie die betriebliche, schulische und hochschulische Bildung. In der Berufsbildungspolitik wird der gesamtgesellschaftliche Kontext der wirtschaftlichen Erziehung kaum beleuchtet. Aktuel- 
le brisante Themen wie persönliche Verantwortung und Korruption, Wirtschaftskrise, Grenzen des Wachstums, Verteilungsgerechtigkeit und Ökonomisierung der Gesellschaft sind untergeordnete Randbereiche, wenn nicht sogar Inhalte, die nicht der Wirtschaftspädagogik zugeordnet werden.

Die Wirtschaftspädagogik stellt sich heute als eine stark ausdifferenzierte und ausdifferenzierende Wissenschaft dar, die sich vor allem auf Institutionen und Strukturen beruft und dabei Gefahr läuft, wesentliche sozioökonomische Grundfragen nicht zu diskutieren. Dabei wäre gerade dieses Fach dafür prädestiniert, über wissenschaftliche Disziplinen hinweg interdisziplinär auf die aktuellen sozio-ökonomischen Fragen unserer Zeit einzugehen.

Adam Smith verstand die Gesetze der Wirtschaft als ,zugleich göttliche und natürliche Rechte" (Rüstow 2009, 22). Wirtschaft folgte seiner Auffassung nach naturgesetzlichen Regeln, die als gottgewollt und damit als moralisch gut einzuhalten waren (vgl. Luterbacher-Maineri 2008, 406f). In diesem Beitrag wird Wirtschaft als ein von Menschen geschaffenes und damit kulturelles Phänomen verstanden. Die Unterscheidung der Wissenschaften in Natur- und Kulturwissenschaften führt zum Schluss: Wirtschaftspädagogik ist eine Kulturwissenschaft (vgl. Schannewitzky 1995, 7; Abraham 1966, 26). „Gesellschaft ist ein menschliches Produkt. Gesellschaft ist eine objektive Wirklichkeit. Der Mensch ist ein gesellschaftliches Produkt" (Berger \& Luckmann 1977, 65). Ein solcher Blick kann aus dem Geist des Cultural Turn neue Perspektiven eröffnen: Ökonomie wird als ein von Menschen geschaffenes System verstanden, das sich in seiner kultureller Bedeutung erschließt und Organisationen werden als in die Gesellschaft eingebettet verstanden.

Ethik, Neo-Institutionalismus und Europapädagogik könnten vor dem Hintergrund dieses Zugangs neue Perspektiven eröffnen und zu einer Neuakzentierung der Wirtschaftspädagogik führen.

\section{Ethik - der Preis der Wirtschaftspädagogik}

Die Positionierungen der Wirtschaftspädagogik sind vielfältig. Aff (2008) arbeitete sechs Positionierungen von der rein wirtschaftswissenschaftlichen bis zur reinen erziehungswissenschaftlichen Zuordnung aus und sieht schließlich die Wirtschaftspädagogik als eigenständige Integrationswissenschaft. In dieser Positionierung ,werden durch die in der Ökonomie bestehenden antinomischen Beziehungen zwischen ökonomischer Rationalität und individuellen Ansprüchen (lebenspraktische Vernunft lt. P. Ulrich) die beiden Pole des Verhältnisses zwischen Wirtschaft und Erziehung, also der zentrale Gegenstandsbereich von Wirtschaftspädagogik, markiert" (Aff 2008, 7). Selbst die 
Vielfalt verschiedener ökonomischer Rationalitäten (vgl. Aff 2004, 28-30) und das Ausgehen von einem „breiten ökonomischen Rationalitätsbegriff“" führe zu einer antinomischen Beziehung zwischen Ökonomie und Pädagogik (Aff 2004, 39). Eine Antinomie ist ein unauflöslicher Widerspruch und für ,jede[n] Widerspruch gilt: Wenn die beiden begrifflichen Bilder [...] sich widersprechen, so heben sie sich gegenseitig auf. An eines von ihnen und an seine Negation zu glauben bedeutet, nichts zu glauben und also kein Bild zu besitzen“ (Bieri 2011, 22). Wäre es so, dann gäbe es entweder keine Wirtschaftspädagogik oder keine Markierung derselben. Es wird wohl so sein, dass es die Wirtschaftspädagogik gibt, sich aber Pädagogik und Ökonomie anders zu einander verhalten. Aff (2008) ordnet der Pädagogik humanistische und der Ökonomie Begriffe wie Effizienz, Produktivität und Gewinnmaximierung zu. Wird Ökonomie so verstanden, dass sie sich ausschließlich auf diese Begriffe reduzieren lässt, dann geht es um einen Ansatz der reinen ökonomischen Ethik, also einer Ökonomie, die sich selbst genügt und im ökonomischen Ziel die Nutzen- und Gewinnmaximierung sieht, die sie selbst zur Norm des ökonomischen Handeln erhebt und eben dadurch zur Ethik selbst wird und einer humanistischen Pädagogik widerspricht. Eine solche Ethik wird im deutschen Sprachraum u.a. von Homann (vgl. Homann 2008, Homann \& Blome-Drees 1992) vertreten und heftig diskutiert (vgl. Aßländer \& Nutzinger 2010, 226-248). Innerhalb der Wirtschaftspädagogik hat diese Diskussion zur Beck-Zabeck-Kontroverse ${ }^{1}$ geführt. Dabei folgt Beck Homanns Ethik und fordert, dass Menschen lernen müssen, sich ihren Rollen gemäß an der Moral des jeweiligen Subsystems zu orientieren. Die Ausdifferenzierung habe dazu geführt, dass eine universalistische Moral im Sinne Kants nicht mehr greife und durch eine partikulare ersetzt werden müsse. Moral müsse immer auf die menschliche Natur Bezug nehmen, ein moralischer Zeigefinger helfe nicht weiter. ${ }^{2}$ (vgl. Beck 2003) Zabeck lehnt auf Basis einer universalistischen Moral Becks „Betriebsmoral“ als partikulare Sondermoral ab. In Homanns Ethik ist der Ort der Moral die Rahmenordnung der Wirtschaft. Das richtige - auch moralisch richtige - Verhalten liegt dann darin, die Rahmenordnungen einzuhalten und den Gewinn bzw. den Nutzen zu maximieren. In dieser Ausformung von ökonomischen Denken und Handeln ist für den wirtschaftenden Menschen bereits alles entschieden.

1 Siehe u.a. dazu in ZBW: Jürgen Zabeck (Heft 4/2002), Helmut Woll (Heft 1/2003), Kurt Beck (Heft 2/2003), Wolfgang Lempert (Heft 3/2003), Gerhard Minnameier (1/2005). Eine ausführliche Behandlung ist hier nicht möglich, erfolgt aber in der sich in Bearbeitung befindlichen Habilitationsschrift.

2 Beck $(2003,276)$ versucht die implizite Seite der Moral quasi-naturwissenschaftlich über Gene und Meme im Sinne Dawkins herzuleiten und begibt sich damit auf einen szientistischen, wissenschaftlich höchst umstrittenen Weg. Der Untertitel des letzten Beitrages des 2005 eingestellten Journals of Memetics spricht für sich: "The revealed poverty of the gene-meme analogy - why memetics per se has failed to produce substantive results" (Edmonds, http://cfpm.org/jom-emit/2005/vol9/edmonds_b.html). 
Es entsteht ein Determinismus, der mit Freiheit nicht vereinbar und keinen Raum für persönliche Entscheidungen und damit keinen für Verantwortung lässt. In einem solchen Fall geht es nicht um einen Widerspruch, sondern um einen absoluten Vorrang der ökonomischen Rationalität, bzw. um eine Absolutsetzung der Ökonomie. Entscheidend ist die Erkenntnis, dass die ökonomische Vorgabe hier als Norm und Ethik, also als Rechtfertigung des eigenen Handelns dient. Aber Ökonomie wird hier verkürzt verstanden: Knappheit wirft immer auch die Frage des Habens und Nicht-Habens sowie des Teilens und Nicht-Teilens auf. Damit ist Knappheit immer auch eine Frage der Gerechtigkeit und daher von sich aus immer auch eine ethische. Die Entwicklung des ökonomischen Denkens und Handelns hat jedoch dazu geführt, dass die Antwort auf die Frage der Knappheit mit Funktionalität, Effizienz und Produktivität beantwortet wird. Die Frage der Gerechtigkeit, die sich ebenso aus der Knappheit ergibt, die für die antiken Griechen bis zu den Gelehrten ins Mittelalter noch eine wesentliche von Ökonomie nicht zu trennende Frage war, ist mit der verkürzten Rezeption des Werkes Adam Smiths und der danach einsetzenden Weiterentwicklung und Herauskristallisierung der Wirtschaftswissenschaften verloren gegangen (vgl. Rolle 2005, 84-186). Samuelson und Nordhaus $(2001,162)$ bringen ein, dass ein funktionierender Markt im besten Fall für Effizienz aber nie für Gerechtigkeit sorgen kann. Wenn es um die Frage der Gerechtigkeit geht, verlassen wir die Ökonomie in dem vorher definierten engen Sinn und wenden uns humanistischen Fragen zu, die für die Pädagogik von höchster Relevanz sind. Pädagogik ist Ausbildung, Bildung und Erziehung. Erziehung ist immer mit Ethik verbunden, denn Erziehung bedarf der Zielsetzung und damit der Rechtfertigung. Ausbildung zielt darauf, Menschen für einen Beruf vorzubereiten und Bildung zielt auf die Selbsterkenntnis. Ausgebildet kann man werden, bilden muss man sich selbst. Sich bilden heißt, sich zu verändern, sich besser kennen zu lernen, seine eigene Wünsche zu verstehen, urteilen zu können und einen Willen auszuformen, den man verantwortlich in Handlungen umsetzt und kritisch reflektierend hinterfragt (vgl. Bieri 2007). Obwohl Ausbildung immer die Beschäftigungsfähigkeit vor Augen hat - sonst wäre sie keine Ausbildung -, muss sie aber immer auch für Bildung offen sein. Eine Verabsolutierung der Employability im Sinne einer Ausgrenzung von Bildung ist nicht möglich, wenn die Förderung von Verantwortungsbewusstsein und die damit einhergehende persönliche Willensbildung ernstgenommen werden.

Kurz gesagt: Es geht um die Fähigkeit des verantwortungsvollen Entscheidens unter gegebenen Bedingungen. Die Willensfreiheit ist in die Bedingungen der Welt eingebettet und damit sind sie die Voraussetzung für Freiheit und daher mit der Freiheit kompatibel. Wer gebildet ist, ist urteilsfähig, kann aus seinem Willen Handlungen entstehen lassen, wenn es die Umstände zulassen. Wird Ökonomie absolut gesetzt, dann gibt es keinen Handlungsspielraum mehr, dann gibt es keinen eigenen Willen mehr, keine 
Freiheit und damit auch keine Möglichkeit mehr, Verantwortung zu übernehmen - das Argument des „Sachzwanges“ wird dann eingebracht. Eine praktische Vorgabe, die Entscheidungen erleichtet, weil eigentlich schon entschieden ist. In einer „,werteunsicheren“ (Brezinka 1986), pluralistischen „Multioptionsgesellschaft" (Gross 2005), in der es immer schwieriger wird, selbst Entscheidungen zu treffen, weil moralische Leitlinien weniger klar sind, ist die ökonomische Komplexitätsreduktion in ihrer simplen reinen ökonomischen Ethik dermaßen verführerisch, dass sie sich mit einer unglaublichen Wucht hat durchsetzen können. Eine Wucht, die bereits Weber nur religiös hat erklären können (vgl. Weber 2006) und später als Auswuchs einer Ökonomie beschrieben wurde, die selbst zur Religion geworden ist (vgl. Baecker 2004, Nelson ${ }^{3}$ 2006, Rüstow 2009). Die reine ökonomische Norm wird tradiert und rezipiert. Eine wesentliche Aufgabe übernehmen dabei Professionen (vgl. Hasse \& Krücken 2005, 25-27), insbesondere Pädagoginnen und Pädagogen. Sie geben laufend Normen weiter, die wiederum auf Individuen und Gesellschaft zurückwirken. Moral, Recht und kulturelle Selbstverständlichkeit lenken das ökonomische Denken und Handeln (vgl. Scott 2001, 47-68), befreien aber nicht von der persönlichen Verantwortung. Die Ökonomie gibt Bedingungen vor, komplexitätsreduziert in Form von finanzierbar und nicht-finanzierbar. In diesem Sinne kann die Ökonomie tatsächlich eine Grenze vorgeben. In gleicher Weise kann auch die Moral Grenzen vorgeben, in dem sie sagt, was man tun und was man unterlassen soll - grundsätzlich bei jeder ökonomischen Handlung.

Die im Mittelpunkt der Beck-Zabeck-Kontroverse stehende Segmentierungs- und Universalisierungsdebatte (vgl. Zabeck 2002, Beck 2003, Fußnote 1) trifft letztlich nicht den wesentlichen Punkt: Ethik erschöpft sich weder in einer deontologischen, vor allem Kant folgenden Ethik, noch in einer utilitaristischen. Es gibt auch eine Ethik, die der Tradition Thomas von Aquin folgend die Strukturganzheit einer Handlung in den Blick nimmt. Nicht nur die Absicht und die Folge, auch die Situation und der Kontext sind dabei zu berücksichtigen. Handeln in diesem Sinne wird Klugheit genannt. Sie geht auch von universellen Überzeugungen aus, weiß aber, dass diese eben situativ angewandt werden müssen. (vgl. Pöltner 2006, 34-47) Die Ausdifferenzierung endet in den Köpfen der Menschen. Sie müssen die Probleme erfassen, verschiedene Perspektiven einnehmen und Lösungen finden. Vor einer Entscheidung gestellt, fließen alle systemischen Betrachtungen zu einem Bild im Kopf zusammen. Ethik ist der Preis der Moderne (in Anlehnung an Höffe 1993), denn nur sie kann das Getrennte wieder zusammenführen. Die pädagogische Herausforderung liegt damit in der Förderung des vernetzten Denkens sowie der ethischen und ökonomischen Entscheidung in

3 Nelson fordert eine „efficient religion“, in der die Volkswirte als Priester das Eigeninteresse in der Wirtschaft als wünschenswert, in anderen sozialen Bereichen jedoch als verpönt predigen. 
komplexen Situationen. Dabei kommen sowohl individual- als auch institutionenethische Überlegungen, je nach Entscheidungssituation, zum Tragen. Ausgangspunkt und Endpunkt jeder Entscheidung soll dabei aus pädagogischer und ethischer Sicht immer der Mensch sein. Damit kommen die universellen Menschenrechte als Wertegeneralisierung (Joas 2011) ins Spiel. Beck spricht sich gegen die „Forderung nach Beachtung der Menschenwürde, nach Gerechtigkeit oder einer wie auch immer definierten Utilität" (Beck 2006, 11) aus, weil sie als Universalethiken regelmäßig wegen ihrer Unbestimmtheit keine moralischen Probleme lösen könnten. Da die Geschichte lehre, dass die Idee des ,guten Menschen“ geschichtlich nicht umsetzbar gewesen sei, sei es notwendig, eine partikulare Ethik einzuführen und eigene Berufsethiken auszuformulieren (vgl. Beck 2006, 21). Die Orientierungslosigkeit solle also nach Beck (vgl. Lempert 2003, 444) dazu führen, dass wesentliche Grundlagen der Pädagogik, des Rechts und der Ethik relativiert, seziert und letztlich abgetragen werden - ein Weg, dem die reflexive Wirtschaftspädagogik nicht folgt. „Moralität zum Nulltarif gibt es nicht!“ (Zabeck 2002, 500) Ethik ist der Preis der Wirtschaftspädagogik.

\section{Wirtschaftliche Erziehung kennt die Grenzen der Wirtschaft}

Ein allgemeines Wirtschafts- und Gesellschaftsverständnis (vgl. Dubs 2009, 9-18) bezieht sich als unverzichtbarer Bestandteil verantwortungsvoller wirtschaftlicher Erziehung und Ausbildung in- und außerschulischer Natur als lebensbegleitende Aufgabe für alle Altersgruppen dem World-Polity-Ansatz des Neo-Instutionalismus folgend (vgl. Meyer et al 2005) auf das Individuum und seine Verantwortung als Betreiber und Betroffener in Organisationen, Staaten und supranationalen Organisationen. Der World-Polity-Ansatz geht davon aus, dass drei Prinzipien des westlichen Denkens die Welt erobert haben: Erstens die Idee des Individuums, dem als rationales und selbstverantwortliches Wesen Menschenwürde und Menschenrechte zukommen. Zweitens die Idee der rationalen Organisation und drittens der rationale Nationalstaat. Doch im Unterschied zu Weber zeigt der Neo-Institutionalismus auf, dass die Rationalität nicht immer und automatisch die Handlungsstruktur darstellt, sondern die Formalstruktur, die Programmatik. Die Handlungen selbst folgen verschiedenen, nicht nur zweckrationalen Motiven. Dies führt $\mathrm{zu}$ einer Entkopplung, die das Individuum in einer Work-Live-Unbalance wahrnimmt, die zu einer immer größeren Problematik unserer Zeit wird. Menschen wollen sich beruflich und privat verwirklichen - beide Motive lassen sich nicht unter den zweckrationalen Vorgaben unter einen Hut bringen. In der Organisation führen implizite, konjunktive, atheoretische Phänomene 
der Unternehmenskultur zu einer Umsetzung der zweckrationalen Vorgaben, die als eine Entkopplung der Handlungsstrukturen von den Formalstrukturen wahrgenommen wird. Auch Nationen versuchen, den allgemeinen Prinzipien der Zweckrationalität zu folgen, doch führt die kulturbedingte Umsetzung ebenso dazu, dass die Praxis nicht der Theorie entspricht. Aber: Alle, Individuen, Organisationen und der Nationalstaat, legitimieren ihr Tun auf Basis der Zweckrationalität. Reines ökonomisches Denken und Handeln ist damit mehr eine Legitimations- als eine tatsächliche Handlungsgrundlage und damit, wie oben ausgeführt, eine normative Vorgabe.

Für die Wirtschaftspädagogik könnten diese Überlegungen wesentliche Auswirkungen haben: Mittel- und Ausgangspunkt der Wirtschaftspädagogik ist der Mensch. Dabei ist die Grundfrage aufzuwerfen, welcher Anthropologie gefolgt wird und welche Rolle der homo oeconomicus als Menschenbild spielt - und dies nicht nur im Modell. Historisch-anthropologische und handlungstheoretische Ansätze zeigen, dass sich das Modell zur Norm entwickelt hat (vgl. Rolle 2005, 357-381). Der Begriff der Organisation ist weiter zu fassen und reduziert sich nicht nur auf das gewinnorientierte Unternehmen, sondern auch auf die Öffentliche Verwaltung, NGO und NPO. Darüber hinaus sind auch der Haushalt und die Konsumentinnen und Konsumenten zu berücksichtigen. Auch hier wird klar, dass es zu aller erst eines Menschenbildes, einer Anthropologie bedarf, bevor weitere Überlegungen angestellt werden können. Neben den ökonomischen Grundfragen eröffnen sich synchron auch ethische, sowohl wirtschafts- als auch unternehmensethische sowie konsumentenethische. Ohne Anthropologie und Ethik kann sich keine Pädagogik rechtfertigen. Schließlich kommt der Staat und aufgrund der Globalisierung und Europäisierung auch die supranationale Ebene ins Spiel.

\section{Europapädagogik - europäische Supranationalität begreifbar machen}

Ökonomisches Handeln hat neben seiner marktwirtschaftlichen immer auch eine ordnungspolitische Dimension. Ein funktionierender Markt braucht eine legitimierte Rahmenordnung und kann im besten Fall, wie bereits dargestellt, nur für Effizienz, aber nicht für Gerechtigkeit sorgen. Das ist national- und suprastaatliche Aufgabe.

Wirtschaftliche Erziehung muss deshalb den Staat und aufgrund der fortschreitenden Globalisierung und Europäisierung auch die supranationale Ebene in die ökonomische Betrachtung mit einbeziehen. Volkswirtschaftliche Zusammenhänge lassen sich heute in Europa nicht ohne den Prozess der europäischen Integration erklären. Und dieser Prozess wiederum kann nicht allein ökonomisch begründet, sondern nur in seiner historischen, real- 
politischen und institutionellen Entwicklung nachvollzogen werden. So ist die Europäische Union heute nichts anderes als das Ergebnis von sechs Jahrzehnten politischer Kompromisse, in der die Wirtschaft für eine intensive Vernetzung der Volkswirtschaften und somit auch der Staaten gesorgt hat (vgl. Tafner 2009, 120). Die Frage nach dem Nutzen der Europäischen Union greift zu kurz: Studien belegen für Österreich den ökonomischen Vorteil (vgl. z.B. Breuss 2010), trotzdem ist die Bevölkerung der europäischen Integration gegenüber äußerst skeptisch (vgl. Europäische Kommission 1995-2011). Die Antworten auf die Fragen nach dem Nutzen verhallen ungehört (vgl. Amt der Steiermärkischen Landesregierung 2006, 13). Eine mögliche Antwort darauf könnte im Nutzen-Identitäts-Paradaxon gefunden werden: Die Unionsbürgerinnen und -bürger stellen die Frage nach den Nutzen nicht, weil sie wirklich darauf eine Antwort hören möchten, sondern weil sie damit implizit zum Ausdruck bringen, dass sie sich mit der EU nicht identifizieren. Welche Österreicherin würde fragen „Was bringt mir Österreich?“, welcher Deutsche würde fragen, „Was bringt mir Deutschland?“. Die Fragen klingen absurd, weil die Nationen Teil der Identität sind, die EU jedoch nicht. Obwohl die Europäische Union rechtlich eindeutig zu definieren ist, fungiert sie doch wie der Begriff Europa als komplexitätsreduzierendes Kommunikationsmittel für verschiedene Vorstellungen und Werthaltungen. Eine pädagogische Auseinandersetzung mit der Europäischen Union kann nicht bei der Identitätsstiftung ansetzen - sie muss andere Wege gehen.

Kein anderes Thema ist als solches so komplex und multidimensional wie dieses, weil sich darin moralische, geschichtliche und wirtschaftspolitische Vorstellung dermaßen miteinander vermengen, dass diese Dimensionen als solche gar nicht erkannt werden. Die Europäische Union und die europäische Idee wiederum sind selbst Institutionen, die unser Denken und Handeln leiten. Durch Isomorphie wird Europarecht in allen Mitgliedsstaaten umgesetzt, verwaltungstechnische, kulturelle und historische Unterschiede führen aber dazu, dass die Programmatik nicht überall gleich umgesetzt und eine Entkopplung stattfindet. Am offensichtlichsten wird dies am Auseinanderklaffen der Ideen von Kooperation, Integration und Menschenrecht einerseits sowie den Diskussionen und Problemen von Migration und Integration andererseits. Die Erklärung dürfte paradoxerweise in den gemeinsamen europäischen Werten liegen, die sich aus verschiedenen Weltanschauungen und Religionen speisen und sich in den unterschiedlichen Handlungsstrukturen der Menschen widerspiegeln: Nationales Selbstinteresse, der Glaube an ein gemeinsames christlich-jüdisches europäisches Erbe und das Festhalten an universalistische Überzeugungen kosmopolitischer oder laizistischer Ausprägung (vgl. Tafner 2010; Mandry 2009) können sowohl der Grund für Kooperation als auch für Nicht-Kooperation, für Integration als auch NichtIntegration sein. Europapädagogik setzt daher nicht bei einer wie immer auch definierten europäischen Identität an, sondern versucht, das Typische und 
Besondere der europäischen Supranationalität begreifbar zu machen. Die EU wird als Problemlösungsplattform verstanden, auf der versucht wird, unter Knappheitsbedingungen und Wahrung von Grund- und Menschenrechten Ziel- und Interessenkonflikte zu lösen (vgl. Tafner 2009, 2010a). Diese Problemlösungsplattform besteht vereinfacht ausgedrückt aus zwei realen Plattformen - dem Rat der Europäischen Union und dem Europäischen Parlament - und einer virtuellen, nämlich den europäischen Zivilgesellschaften, die aus ihrem nationalstaatlichen Kontext heraus beginnen sich transnational in die europäische Diskussion einzubringen.

Vor dem Hintergrund eines solchen undurchsichtigen Gemenges ist die Vermittlung europäischer Inhalte und die Förderung von Europakompetenz zu einer außergewöhnlich schwierigen und herausfordernden pädagogischen Aufgabe schulischer und außerschulischer Art geworden. Hier muss wirtschaftliche Erziehung Vorgaben geben: Menschenrechte, Frieden, Demokratie, Freiheit und Rechtsstaatlichkeit werden zum Ausgangspunkt und grundsätzlichem Ziel, die Ergebnisse der Problemlösungsplattform und ihre gegenwärtige Struktur gilt es kritisch zu hinterfragen. Europapädagogik setzt der Komplexität entsprechend multidimensional an und spricht von der $3^{3}$ Formel: Interdisziplinär werden die Bereiche Politik, Wirtschaft und Recht für Hand, Kopf und Herz für drei Lernzielniveaus - reproduzieren, anwenden und konstruieren - aufgearbeitet.

\section{Reflexive Wirtschaftspädagogik}

Die Auseinandersetzung mit Europa zeigt, dass hinter gemeinsamen Werten verschiedene Denk- und Handlungsweisen sowie Weltanschauungen und Religionen stehen können. Wirtschaftspädagogik, die nicht indoktriniert, muss Raum für verschiedene weltanschauliche und ethische Zugänge auf Basis einer von Menschenrechten getragenen pluralistischen Gesellschaft lassen und sie muss auch die Relativität des ökonomischen Handelns selbst thematisieren. Ökonomische Erziehung und Ausbildung nimmt den Menschen als Betreiber und Betroffenen in den Blick und stellt die Verantwortung sich selbst (vgl. Roth 1971, 180) und der Gesellschaft gegenüber in den Mittelpunkt. So gilt es zu hinterfragen, inwiefern die Wirtschaftspädagogik explizit und implizit eine Arbeitsmoral fördert, die einem reinen ökonomischen Ansatz folgt, obwohl sie selbst in ihrer pädagogischen Seite auf den Menschen abstellen will. Die Wirtschaftspädagogik kann zu einer echten Integrationswissenschaft werden, wenn sie die ökonomische Anthropologie neu fasst und den Menschen in seiner Gesamtheit berücksichtigt.

Reflexionsfähigkeit zeichnet ausdifferenzierte Gesellschaften aus (vgl. Douglas 1985, 100-105) - das sollte auch für die Ökonomie und noch viel 
mehr für die Wirtschaftspädagogik gelten. Reflexive Wirtschaftspädagogik will daher in nuce:

- $\quad$ eine vertiefte Reflexion über Sinn und Ziel der Ökonomie und der Wirtschaftspädagogik sowie eine wirtschaftliche Erziehung zwischen ökonomisch Machbarem und ethisch Vertretbarem,

- Ökonomie als einen Bestandteil der Gesellschaft verstehen, der den Menschen dient (und nicht umgekehrt),

- die sozio-ökonomischen Zusammenhänge auf individueller, organisatorischer, staatlicher und suprastaatlicher Ebene, insbesondere europäischer Ebene, aufzeigen,

- $\quad$ sich in diesem Sinne als sozio-ökonomische Bildung für alle verstehen,

- aufzeigen, dass die Ökonomie nicht alle Probleme lösen kann und dies auch entsprechend vermittelt,

- frei sein von politischer, insbesondere wirtschaftspolitischer Indoktrination auf Basis einer von Menschrechten getragenen, pluralistischen Grundeinstellung.

Denn reflexive Wirtschaftspädagogik ist nicht nur eine Erziehung für die Wirtschaft, sondern auch eine „Erziehung der Wirtschaft“"

\section{Literatur}

Abraham, Karl (1966): Wirtschaftspädagogik. Grundfragen der wirtschaftlichen Erziehung. 2. Aufl. Heidelberg: Quelle \& Meyer.

Aff, Josef (2004): Wirtschaftsdidaktik zwischen ökonomischer Rationalität und pädagogischem Anspruch. In: Zeitschrift für Berufs- und Wirtschaftspädagogik, 100. Band, Heft 1. S. 26-42.

Aff, Josef (2008): Pädagogik oder Wirtschaftspädagogik? Anmerkungen zum Selbstverständnis der Disziplin. In: Gramlinger, F./Schlögl, P./Stock, M. (Hrsg.): bwp@- Österreich Spezial: Berufs- und Wirtschaftspädagogik in Österreich, Spezial 3, S. 1-14. Online verfügbar unter www.bwpat.de/ATspezial/aff_at spezial.pdf, zuletzt geprüft am 21.10.2011.

Amt der Steiermärkischen Landesregierung (2006): EUGEM - EUropa GEMeinde Steiermark. Endbericht - Juli 2006. Graz. Online verfügbar unter http://www. europa.steiermark.at/cms/dokumente/10260778_5225562/29de861a/EUGEMEndbericht.pdf, zuletzt geprüft am 02.11.2011.

Aßländer, Michael S.; Nutzinger, Hans G. (2010): Der systematische Ort der Moral ist die Ethik! Einige kritische Anmerkungen zur ökonomischen Ethik Karl Homanns. In: Zeitschrift für Wirtschafts- und Unternehmensethik (zfwu) 11 (3), S. 226-248. 
Baecker, Dirk (Hrsg.) (2004): Kapitalismus als Religion. 2. Aufl. Berlin: Kulturverl. Kadmos (Copyrights, 9), S. 15-18.

Beck, Klaus (2003): Ethischer Universalismus als moralische Verunsicherung? In: Zeitschrift für Berufs- und Wirtschaftspädagogik, 99. Band, Heft 2. S. 274-298.

Beck, Klaus (2006): Relativismus und Rolle - Zur Grundlegung einer differentiellen Moralerziehung. In: Gonon, Philipp; Klauser, Fritz; Nickolaus, Reinhold (Hrsg.): Bedingungen beruflicher Moralentwicklung und beruflichen Lernens. Wiesbaden: VS Verlag für Sozialwissenschaften, S. 9-22.

Berger, Peter L.; Luckmann, Thomas (1977): Die gesellschaftliche Konstruktion der Wirklichkeit. Eine Theorie der Wissenssoziologie. 5. Aufl. Frankfurt/M.: Fischer.

Bieri, Peter (2007): Wie wäre es, gebildet zu sein? Grünwald: Komplett-Media.

Bieri, Peter (2011): Das Handwerk der Freiheit. Über die Entdeckung des eigenen Willens. 10. Aufl. Frankfurt/M: Fischer.

Breuss, Fritz (2010): Österreich 15 Jahre EU Mitglied. In: WIFO Monatsberichte (2), S. $117-136$.

Brezinka, Wolfgang (1986): Erziehung in einer wertunsicheren Gesellschaft. Beiträge zur praktischen Pädagogik. 2. Aufl. München: Reinhardt.

Douglas, Mary (1985): Reinheit und Gefährdung. Eine Studie zu Vorstellungen von Verunreinigung und Tabu. Berlin: Reimer.

Dubs, Rolf (2009): Finanz- und Wirtschaftskrise. Die Bedeutung der wirtschaftlichen Bildung. In: Michaela Stock (Hrsg.): Entrepreneurship, Europa als Bildungsraum, europäischer Qualifikationsrahmen. Tagungsband zum 3. Österreichischen Wirtschaftspädagogik-Kongress. Graz. Wien: Manz, S. 9-18.

Europäische Kommission (1995-2011): Standard Eurobarometer. Online verfügbar unter http://ec.europa.eu/public_opinion/archives/eb_arch_en.htm, zuletzt geprüft am 10.01.2012.

Gross, Peter (2005): Die Multioptionsgesellschaft. Frankfurt/M.: Suhrkamp.

Hasse, Raimund; Krücken, Georg (2005): Neo-Institutionalismus. 2. Aufl. Bielefeld: Transcript-Verl.

Höffe, Otfried (1993): Moral als Preis der Moderne: Ein Versuch über Wissenschaft, Technik und Umwelt. Berlin. Suhrkamp.

Homann, Karl; Blome-Drees, Franz (1992): Wirtschafts- und Unternehmensethik. Göttingen: Vandenhoeck \& Ruprecht.

Homann, Karl (2008): Die Ethik in der Wirtschaft. In: Hermann May (Hrsg.): Lexikon der ökonomischen Bildung. 7. Aufl. München: Oldenbourg, S. 199-201.

Lempert, Wolfgang (2003): Modernisierung der Moral oder pseudomoralische Entmoralisierung? In: Zeitschrift für Berufs- und Wirtschaftspädagogik, 99. Band, Heft 3. S. 436-452.

Joas, Hans (2011): Die Sakralität der Person. Eine neue Genealogie der Menschenrechte. Berlin: Suhrkamp.

Lisop, Ingrid (2009): Identität und Krisenanfälligkeit der Berufs- und Wirtschaftspädagogik im Spiegel der Kategorien Kompetenz und Employability. In: bwp@ Berufs- und Wirtschaftspädagogik-online, Ausgabe 16, 1-18. Online verfügbar unter www.bwpat.de/ausgabe16/lisop_bwpat16.pdf, zuletzt geprüft am 21.10.2011.

Luterbacher-Maineri, Claudius (2008): Adam Smith - Theologische Grundannahmen. Freiburg: Academic Press Fribourg. 
Mandry, Christof (2009): Europa als Wertegemeinschaft. Eine theologisch-ethische Studie zum politischen Selbstverständnis der Europäischen Union. Baden-Baden: Nomos-Verl.-Ges (Denkart Europa, 9).

Meyer, John W.; Krücken, Georg; Kuchler, Barbara (2005): Weltkultur. Wie die westlichen Prinzipien die Welt durchdringen. Frankfurt/M.: Suhrkamp.

Nelson, Robert H. (2006): Economics as religion. From Samuelson to Chicago and beyond. 3. Aufl. University Park, Pa: Pennsylvania State Univ. Press.

Pöltner, Günther (2006): Grundkurs Medizin-Ethik. 2. Auflage. Wien: Facultas.

Prange, Klaus (2000): Plädoyer für Erziehung. Baltmannsweiler: Schneider Verlag.

Rolle, Robert (2005): Homo oeconomicus. Wirtschaftsanthropologie in philosophischer Perspektive. Würzburg, Regensburg: Königshausen \& Neumann.

Roth, H. (1971): Pädagogische Anthropologie (Band II). Entwicklung und Erziehung. Grundlagen einer Entwicklungspädagogik. Hannover: Hermann Schroedel.

Rüstow, Alexander (2009): Die Religion der Marktwirtschaft. 3. Aufl. Berlin, Münster: Lit (Reihe Zweite Aufklärung, 4).

Samuelson, Paul Anthony; Nordhaus, William D. (2001): Economics. 17. Aufl. Boston, Mass: McGraw-Hill/Irwin.

Schannewitzky, Gerhard (1995): Kulturwissenschaftliche Aspekte der Berufs- und Wirtschaftspädagogik. Frankfurt/M.: Lang.

Scott, William Richard (2001): Institutions and organizations. 2. Aufl. Thousand Oaks: Sage.

Tafner, Georg (2009): Europapädagogik - ein Thema legt sich quer. Eine Querschnittsmaterie als neuer Teilbereich der Wirtschaftspädagogik. In: Michaela Stock (Hrsg.): Entrepreneurship, Europa als Bildungsraum, europäischer Qualifikationsrahmen. Tagungsband zum 3. Österreichischen Wirtschaftspädagogik. Graz. Wien: Manz, S. 119-127.

Tafner, Georg (2010): Das islamische Kopftuch: Brennpunkt des verschleierten Kampfes um die europäische Identität. Eine europapädagogische Kurzbetrachtung. In: Österreichisches Archiv für Recht \& Religion 57 (1), S. 98-119.

Tafner, Georg (2010a): Die EU ist kein Maikäfer. Ein Kurzplädoyer für die Europapädagogik als Fachdidaktik. In: Wissenplus, Sonderausgabe Wissenschaft, 509/10, S. 37-40.

Thoma, Michael (2011): Entwürfe des wirtschaftspädagogischen Subjekts. AndersKonzeption aus poststrukturalistischer Perspektive. Wiesbaden: VS Verlag für Sozialwissenschaften.

Weber, Max (2006): Die protestantische Ethik und der "Geist" des Kapitalismus. München: FinanzBuch-Verl (CapitalBuch, 6).

Wöhe, Günter; Döring, Ulrich (2010): Einführung in die allgemeine Betriebswirtschaftslehre. 24. Aufl. München: Vahlen.

Zabeck, Jürgen (2002): Moral im Dienste betrieblicher Zwecke? Anmerkungen zu Klaus Becks Grundlegung einer kaufmännischen Moralerziehung. In: Zeitschrift für Berufs- und Wirtschaftspädagogik, 98. Band, Heft 4/2002, S. 485-503. 


\title{
Betriebspraktika unter dem Aspekt der Arbeitszufriedenheit - eine kritische Auseinandersetzung
}

\author{
Markus Ammann
}

\section{Die Perspektive der Arbeitszufriedenheit}

Die Einbettung betrieblicher Praxisphasen - in diesem Beitrag Betriebspraktika - in Curricula gewinnt zunehmend mehr an Bedeutung, insbesondere in vollzeitschulischen Berufsausbildungen. Deshalb erstaunt es, dass sich nur sehr wenige Arbeiten mit der Frage auseinandersetzen, was ein , qualitätsvolles' Betriebspraktikum genauer auszeichnet (vgl. bspw. Ammann/ Thoma 2011). Die Ausgestaltung, qualitätsvoller' Betriebspraktika und insbesondere auch die Frage nach der didaktischen Begleitung stellt das Lernen und die darauf bezogene didaktische Rahmung im Wechsel unterschiedlicher Lernorte ins Zentrum der Betrachtung (vgl. dazu bspw. van Buer/ Troitschanskaja 2002; van Buer/Troitschanskaja/Höppner 2004; Ostendorf 2007; Heffeter 2008, Kremer/Gockel 2010; Ammann/Waltl 2010). Die Annahmen bezüglich eines Qualitätsmodells für Betriebspraktika bleiben bisher vage und es kann maximal von Indizien gesprochen werden (vgl. Ammann/Thoma 2011). Gleichwohl erscheint es lohnenswert, diesen Indizien nachzugehen und sie auch vor dem Hintergrund anderer Zugänge bzw. Modelle - wie bspw. jene der Arbeitszufriedenheit - zu analysieren. Betriebspraktika können als zeitlich befristete Lern- und Arbeitsszenarien betrachtet werden, mit dem Ziel, die Entwicklung beruflicher Handlungskompetenz zu fördern (vgl. Ostendorf 2008: 19). Insofern können diese zumindest auch, wenngleich nicht ausschließlich, als Arbeitsverhältnisse charakterisiert werden. Die diesbezüglich zu explizierende Annahme wäre, dass zufriedene Praktikanten im Vergleich zu unzufriedenen einen Mehrwert aus dem Betriebspraktikum ziehen und somit das Praktikum in Summe als erfolgreicher eingestuft werden kann.

Arbeit und damit einhergehend die Frage nach Zufriedenheit mit dieser, zählt zu einer sehr ausführlich untersuchten Thematik in der Arbeits- und Organisationspsychologie (vgl. Nerdinger 2011: 395). Die wissenschaftliche Diskussion zur Arbeitszufriedenheit kann in ihren Grundfesten bis auf Fragen der Arbeitsprozessorganisation und der damit einhergehenden vermeintlich optimalen Gestaltung von Arbeitsplätzen im frühen 20. Jhd. zurückgeführt werden (vgl. Weinert 2004: 247; Kirchler/Hölzl 2008: 246; Marcus 2011: 
27). So ging bspw. Frederick Winslow Taylor - bekannt geworden für das sogenannte Scientific Management - davon aus, dass sich ein langfristig optimales Pensum an Arbeitsleistung einerseits positiv auf die Gesundheit der Arbeiter auswirkt und andererseits durch das vermeintliche Optimum die Maximalleistung in der Produktion erbracht werden könne. Darüber hinaus war er der Meinung, dass seine Arbeiter durch monetäre Anreize motiviert werden können, was als das Prinzip des Pensumlohns bezeichnet wird (vgl. Marcus 2011: 27). Die Ursprünge der Frage nach Arbeitszufriedenheit zeigen schon deutlich, dass - und diese Überlegungen sind bis heute untrennbar mit dem Thema verbunden - Arbeitszufriedenheit auch eine Frage der Motivation, der Mitarbeiterführung und letztlich auch der Ausgestaltung von Arbeitsplätzen ist. Zahlreiche Erhebungsinstrumente wurden entwickelt, wobei im deutschsprachigen Raum insbesondere die ,Skala zur Messung der Arbeitszufriedenheit" (SAZ) (Fischer/Lück 1972) und der ,Arbeitsbeschreibungsbogen' (AAB) (Neuberger/Allerbeck 1978) gewisse Prominenz erlangten.

Wenngleich die verschiedenen Studien und Ergebnisse nicht verglichen werden können, da diese sich auf verschiedene theoretische Hintergründe und Modelle wie bspw. Zwei-Faktoren-Theorie nach Herzberg, AnreizBeitrags-Theorie, Stresstheoretische Überlegungen oder das Arbeitszufriedenheits-Modell von Bruggemann beziehen (vgl. $\mathrm{zu}$ den verschiedenen Zugängen ausführlicher bspw. Nerdinger 2011: 396 ff.; Baumgartner/Udris 2006: 111 ff; Weinert 2004: 197 ff.; Matiaske/Mellewigt 2001: 10 ff), so konnten doch einigermaßen stabile Dimensionen identifiziert werden, die auf die individuell empfundene Arbeitszufriedenheit rückschließen lassen und als Basis der Messung von Arbeitszufriedenheit herangezogen werden. Deshalb eignet sich diese Perspektive m. E. auch sehr gut, um Betriebspraktika vor diesem Hintergrund zu diskutieren.

Die Dimensionen beschäftigen sich mit folgenden Themen (vgl. Weinert 2004: 257): (1) Fragen, welche ,Arbeit' selbst betreffen, (2) Supervision bzw. dem Führungsstil in Organisationen, (3) Organisation und Organisationsleitung, (4) Beförderungsmöglichkeiten, (5) Arbeitskollegen, (6) Arbeitsbedingungen, (7) finanzielle und nicht finanzielle Be- und Entlohnung und (8) Anerkennung. Der vorliegende Beitrag greift diese Überlegungen auf und geht somit zusammenfassend der Frage nach, inwieweit Ansätze der Arbeitsund Organisationspsychologie, die sich mit Fragen der Arbeitszufriedenheit auseinandersetzen Hinweise zur qualitätsvollen und somit zur Zufriedenheit der Praktikantinnen gereichenden Ausgestaltung von Betriebspraktika liefern können.

Zu diesem Zweck wird in der Folge zunächst der mögliche Beitrag zu einem Qualitätsmodell für Betriebspraktika dargestellt und daran anschließend vor dem Hintergrund zweier möglicher ausgewählter Zugänge der potentielle 
Beitrag dieser Perspektive kritisch diskutiert. Der Beitrag endet mit einem vorläufigen Ausblick, bei dem Chancen und Grenzen aufgezeigt werden.

\section{Ein möglicher Beitrag zur Entwicklung eines Qualitätsmodells}

Basis der Diskussion stellen ausgewählte Elemente des sogenannten ,Qualitätsindizientableaus' (vgl. Ammann/Thoma 2011) dar. Dieses war das Ergebnis einer qualitative Interviewstudie mit fünf Absolventen von Betriebspraktika und einer Evaluationsstudie bei der ca. 1.400 Absolventen befragt wurden, von denen ca. $80 \%$ das Praktikum im touristischen bzw. gastronomischen Bereich absolvierten. Die Evaluationsstudie wurde von der zuständigen Landesschulbehörde durchgeführt, welche die Daten zur Verfügung gestellt hat. Neben quantitativen Elementen, beinhaltet die Studie auch qualitative Angaben zu den Praktika. Die Interviews und die qualitativen Aussagen der Evaluationsstudie zu den Praktika, wurden im Rahmen eine qualitativen Inhaltsanalyse nach Mayring (vgl. Mayring 2000) ausgewertet und vor dem Hintergrund der Tätigkeitstheorie nach Engeström (vgl. bspw. Engeström 2004) analysiert. Im Rahmen von Betriebspraktika tauchen Praktikanten für einen - im Idealfall - vorher vereinbarten Zeitraum in die betriebliche Umwelt ein, um dort in die verschiedenen Wertschöpfungsprozesse eingebunden zu werden.

Die (Weiter-) Entwicklung von beruflicher Handlungskompetenz in Betriebspraktika (vgl. Ostendorf 2008: 19) - so die These - gelingt über das Ausüben bzw. Übernehmen berufsfeldbezogener Tätigkeiten am Arbeitsplatz. Mit Engeström kann der Wechsel zwischen verschiedenen Lernorten konzeptionell als Wechsel verschiedener Tätigkeitssysteme verstanden werden (vgl. Kerosuo/ Engeström 2003, 346). Dementsprechend ist ein Tätigkeitssystem ein Bereich oder Aufgabenfeld im Praktikum, welches von verschiedenen Elementen geprägt wird und in dem der Praktikant gegenstandsbezogene Tätigkeiten ausführt: Ein Subjekt, welches an einem spezifischen Gegenstand arbeitet und sich vermittelnder Artefakte bedient. Eingebettet sind die verschiedenen Tätigkeiten in ein spezifisches System von Regeln, die das Tätigkeitsystem regulieren. Ausgeführt werden die verschiedenen Tätigkeiten in einer Gemeinschaft von anderen Kollegen, welche sich im Rahmen einer spezifischen Arbeitsteilung am selben Gegenstand beteiligen (vgl. Engeström 2008, 63). Diese Elemente bildeten die theoretische Basis für die Analyse der Daten. Das daraus resultierende Ergebnis war das sogenannte Qualitätsindizientableau, welches einzelne Dimensionen und Kriterien abbildet, welche eben - wie der Name impliziert - Hinweise auf die qualitätsvolle Ausgestaltung von Betriebspraktika liefern kann (vgl. Am- 
mann/Thoma 2011). Ausgewählte Ergebnisse dieser Studie stellen die Basis der weiterführenden Diskussionen in diesem Beitrag dar und sollen - wie oben bereits angedeutet - aus der Perspektive verschiedener Überlegungen zur Arbeitszufriedenheitsforschung diskutiert werden.

\section{Tabelle 1: Qualitätsindizientableau}

\begin{tabular}{|c|c|c|}
\hline Rahmenbedingungen & Unternehmenskultur & Handlungsfeld \\
\hline Ort - Region & Umgangsformen & Selbstständigkeit \\
\hline Hygiene am Arbeitsplatz & Gemeinschaftsgefühl & $\begin{array}{l}\text { Form der Tätigkeiten } \\
\text { - Job Rotation }\end{array}$ \\
\hline Unterkunft & $\begin{array}{l}\text { Gemeinsame Freizeitakti- } \\
\text { vitäten }\end{array}$ & $\begin{array}{l}\text { Über- bzw. Unterfor- } \\
\text { derung }\end{array}$ \\
\hline Niveau des Betriebes & Kommunikation & $\begin{array}{l}\text { Eigene Entschei- } \\
\text { dungsräume }\end{array}$ \\
\hline MitarbeiterInnenstand & Peers & Zusatzaktivitäten \\
\hline Bezahlung & MitarbeiterInnenführung & Verantwortung \\
\hline Ansprechpartner & Verpflegung & $\begin{array}{l}\text { Instruktion durch } \\
\text { Kolleginnen }\end{array}$ \\
\hline $\begin{array}{l}\text { Vor- und Nachbereitung, } \\
\text { Begleitung }\end{array}$ & Zeiteinteilung & $\begin{array}{l}\text { Integration in den Ar- } \\
\text { beitsprozess }\end{array}$ \\
\hline
\end{tabular}

Rahmenbedingungen, Unternehmenskultur und Handlungsfelder konnten im Rahmen der Untersuchung als die drei Hauptdimensionen identifiziert werden, in denen sich die verschiedenen Kriterien die auf Qualität hindeuten subsumieren lassen (vgl. Ammann/Thoma 2011). Diese drei Dimensionen scheinen sich zunächst auch deutlich in den stabilen Dimensionen der Arbeitszufriedenheit (vgl. Weinert 2004: 257) zu spiegeln und bilden die Basis der folgenden Diskussion. Aus Platzgründen erfolgt diese in der Folge einerseits aus der Perspektive ,Motivation' und andererseits aus der Perspektive der, Anreiz-Beitrags Theorie'. 


\section{Motivation als Triebfeder}

Es gibt zahlreiche Definitionen, mit denen versucht wird, das an sich außerordentlich komplexe Konstrukt Arbeitszufriedenheit zu definieren. So macht von Rosenstiel bspw. folgenden Vorschlag: „Arbeitszufriedenheit (...) wird meist anders verstanden, nicht als eine stets schwankende, sondern als eine recht stabile Variable. Es handelt sich dabei um eine für die Person kennzeichnende Einstellung der Arbeitssituation gegenüber, um eine aus der Erfahrung kommende Wertung." (von Rosenstiel 2003: 219) Was in diesem Definitionsversuch m. E. deutlich wird, ist der Umstand, dass es sich um die Perspektive des Individuums handelt oder anders gewendet, dass der einzelne Mitarbeiter Zufriedenheit empfinden sollte und ihm diese gewissermaßen nicht extern zugeschrieben werden kann. Als Konsequenz ist der Mitarbeiter dann in der Lage die entsprechende Leistung zu erbringen, was in verschiedenen Definitionen zum Ausdruck kommt. So wird in weiteren Diskussionen nach wie vor die Verbindung zwischen Zufriedenheit und Leistung hergestellt. „Arbeitszufriedenheit korreliert positiv mit Leistung und negativ mit Fehlzeiten und Kündigungshäufigkeit und führt zu einer höheren Bindung an den Betrieb.“ (Kirchler/Hölzl 2008: 242). Diese Überlegungen sind mit Fragen der Motivation verbunden, wie sie bspw. im Rahmen der „ZweiFaktoren-Theorie" nach Herzberg et al. (1959) problematisiert werden.

Die Theorie, welche ursprünglich als ,Zweifaktorentheorie der Arbeitszufriedenheit" konzipiert war (vgl. von Rosenstiel 2007: 88) stellt den Versuch dar, Aussagen zur Gestaltung von Arbeitsplätzen zu machen. Neben ,Hygiene-Faktoren', bspw. Führungsstil, Unternehmenspolitik und -verwaltung oder Beziehungen zu Gleichgestellten, werden auch so genannte ,Motivatoren' wie Leistung, Anerkennung, Verantwortung bzw. die Arbeit selbst (vgl. von Rosenstiel 2007: 89) als zentrale Elemente für die Gestaltung von Arbeitsplätzen angeführt. Hygienefaktoren beinhalten Elemente, die mit dem Arbeitsumfeld verbunden sind und deren Präsenz nicht zu mehr Zufriedenheit führen, die Abwesenheit führt aber zu Unzufriedenheit. Zufriedenheit bzw. zusätzliche und mehr Zufriedenheit wird von den sogenannten Motivatoren erzeugt. „Hygiene operates to remove health harards from the environment of man." (Herzberg et al. 1959: 113) Fallen die Motivatoren wieder weg, so entsteht nicht automatisch Unzufriedenheit sondern vielmehr entsteht wieder ein neutraler Status der ,Nicht-Zufriedenheit ' (vgl. von Rosenstiel 2007: 88 ff.). „It should be understood that both kinds of factors meet the needs of the employee; but it is primarily the ,motivators' that serve to bring about the kind of job satisfaction (...).“ (Herzberg 1959: 114) Wenngleich dieser Ansatz auch kritisch betrachtet wurde und wird, so genießt er doch bis heute hohe Verbreitung in der Arbeits- und Organisationspsychologie, insbesondere auch deshalb, weil er von der Idee ausgeht, dass Menschen nicht ausschließlich durch finanzielle (ökonomische) Mittel motiviert werden kön- 
nen und somit auch andere Wege zu Zufriedenheit führen (vgl. Nerdinger 2011: 398). „Trotz der Kritik sei angemerkt, dass es das große Verdienst von Herzberg et al. (1959) ist, die Aufmerksamkeit der Forschung auf den Inhalt der Arbeitstätigkeit gerichtet und die Reichhaltigkeit der Arbeitsgestaltung als Voraussetzung für Zufriedenheit und Selbstentfaltung betont zu haben.“ (Kirchler/Hölzl 2008: 246)

Betrachtet man die Ergebnisse der Studie zur qualitätsvollen Ausgestaltung von Betriebspraktika, so könnten (!) die gewonnenen Kriterien (vgl. Ammann/Thoma 2011) den beiden Faktoren folgendermaßen zugeordnet werden:

\section{Tabelle 2: Qualitätskriterien für Betriebspraktika - eine Zuordnung}

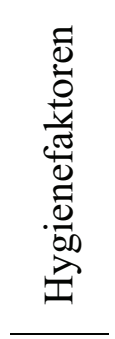

$$
\begin{aligned}
& \text { MitarbeiterInnenführung, Verpflegung, Integra- } \\
& \text { tion in den Arbeitsprozess, Peers, Hygiene am } \\
& \text { Arbeitsplatz, Ansprechpartner, Instruktion durch } \\
& \text { Kolleginnen, MitarbeiterInnenstand, Vor- und } \\
& \text { Nachbereitung, Begleitung, Umgangsformen, } \\
& \text { Gemeinschaftsgefühl, Kommunikation, Niveau } \\
& \text { des Betriebes, Über- bzw. Unterforderung. }
\end{aligned}
$$

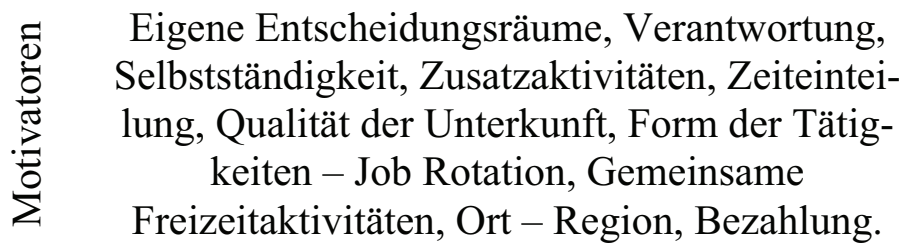

Obwohl es Kritik an der Herzbergschen Theorie gibt, so ist es m. E. doch einigermaßen erstaunlich, dass - und die Theorie war, um es nochmals explizit zum Ausdruck zu bringen, nicht die Basis der Datenauswertung oder in irgendeiner anderen Form Teil der Studie - sich die verschiedenen Kriterien mit diesem Ansatz erklären und argumentieren lassen. Teilweise sind diese mit den verschiedenen von Herzberg genannten Faktoren sogar deckungsgleich (vgl. dazu bspw. Nerdinger 2011: 396 ff. oder von Rosenstiel 2007: 89 f.). Diese Überlegungen lassen m. E. zumindest zwei Annahmen bzw. Vermutungen zu, die in der Folge kurz entfaltet werden können. Einerseits wäre dies möglicherweise als Hinweis dahingehend zu interpretieren, dass Betriebspraktika, obwohl diese als Lern- und Arbeitsverhältnisse betrachtet werden, diesem Anspruch vordergründig nicht gerecht werden und es sich vielfach und mancherorts trotzdem um ,reine' Arbeitsverhältnisse zu handeln 
scheint. Damit ist aber keineswegs die Annahme verbunden, dass durch das Ausführen von Tätigkeiten - arbeiten - nicht auch gelernt wird, vielmehr geht es darum, dass möglicherweise wenig systematische und didaktische Begleitung geschieht. Dennoch scheinen die Herzbergschen Überlegungen auch interessante Hinweise für die qualitätsvolle Gestaltung von Betriebspraktika zu liefern. Insbesondere der Ansatz, dass es auch für betriebliche Praxisphasen eine Kombination von Hygienefaktoren und Motivatoren geben kann, erscheint diesbezüglich von besonderem Interesse.

Die Zuordnung der einzelnen Elemente zu den Faktoren muss andererseits allerdings kritisch betrachtet werden, was auch eine Limitierung der Übertragbarkeit mit sich bringen könnte. So ordnet Herzberg bspw. die Bezahlung (Gehalt, Entlohnung) den Hygienefaktoren zu, was im Rahmen von Betriebspraktika nicht in der Form zutreffend ist. Betriebspraktika werden in den wenigsten Fällen derart ent- und belohnt, wie dies in regulären Arbeitsverhältnissen der Fall ist. Schon alleine aus dieser Überlegung heraus kann diese folglich nicht als Hygienefaktor klassifiziert werden, der bei Abwesenheit zu Unzufriedenheit führt, m. E. wohl aber als Motivator, der zusätzliche Zufriedenheit bringt, wenn entsprechend monetäre Anreize geschaffen werden.

\section{Geben ist nicht immer seliger denn Nehmen}

Matiaske/Mellewigt sehen in der Anreiz-Beitrags-Theorie einen geeigneten Ausgangspunkt, um über Zufriedenheit am Arbeitsplatz nachzudenken (vgl. Matiaske/Mellewigt 2001: 10). Ideengeschichtlich geht die Anreiz/Beitragstheorie auf Barnard (in diesem Beitrag zitiert nach 1968) zurück. Er versuchte im Jahr 1938 erstmals ein Unternehmen als Koalition aller an ihm Partizipierenden zu denken, was später auch als Argumentationsgrundlage für die Begründung des Stakeholderansatzes diente. „Diese ,Beteiligten’ (die Partizipierenden, M.A.) sind solange zur Aufrechterhaltung der Koalitionen bereit, als die von der Koalition gewährten Anreize den Nutzenentgang der an die Koalition geleisteten Beiträge zumindest aufwiegen." (Stahl 2003: 31) In den fünfziger Jahren des vergangenen Jhd. wurde dieser Ansatz von March und Simon aufgegriffen, die diese Überlegungen auch auf die Teilnahme bzw. Austrittsentscheidungen von Organisationsmitgliedern übertrugen (vgl. Matiaske/Mellewigt 2001: 10). „An organization is a system of interrelated social behaviors of a number of persons whom we shall call the participants in the organization." (March/Simon 1958: 84) Die Entscheidungen zur produktiven Beitragsleistung unterschiedlicher Gruppierungen werden in das Zentrum der Überlegungen gestellt. „... March (konzeptualisiert, MA) Organisationen als politische Koalitionen, wobei jeweils die Zusammensetzung 
der Koalition genauso wie die Ziele der Firma erst ausgehandelt werden." (Alt 2001: 289)

Der daraus abgeleitete Gedanke folgt dem Ansatz des Soll-Ist-Vergleichs, der als Basis der empfundenen Arbeitszufriedenheit dient. Diesem Zugang zufolge stellt der Mitarbeiter einen Vergleich zwischen gegebenen Belohnungen (Ist-Zustand) und den erwarteten Belohnungen (Soll-Zustand) an (vgl. Kirchler/Hölzl 2008: 250). Diese Gegenüberstellung kann zu einem günstigen Ergebnis (stabilisierende Zufriedenheit) oder zu einem ungünstigen Ergebnis (diffuse Unzufriedenheit) führen. Im Modell der Arbeitszufriedenheit von Bruggemann werden diese Überlegungen anschaulich dargestellt und umgesetzt (vgl. Baumgartner/Udris 2006: 114 ff.). Je nach Ergebnis des Vergleichsprozesses (günstiges oder ungünstiges Ergebnis) wird der Akteur in diesem Falle der Praktikant - verschiedene Strategien wählen, um mit der Situation umzugehen und diese zu meistern. In Summe können sechs mögliche Strategien (vgl. Baumgartner/Udris 2006: 114) identifiziert werden, welche von den Akteuren zur Bewältigung gewählt werden und zu verschiedenen Zufriedenheitszuständen führen. Bei einem günstigen Ergebnis Erhöhung der Ansprüche oder Beibehaltung der Ansprüche. Bei einem ungünstigen Ergebnis: Senkung der Ansprüche, Verfälschung der Situation, Verharren ohne Problemlösungsversuche bzw. neue Problemlösungsversuche. Bleibt der Zustand der langfristigen Unzufriedenheit bzw. eines Ungleichgewichts zwischen Erwartungen und tatsächlich erhaltener Belohnung aufrecht, wird der Mitarbeiters sich nach alternativen Lösungsmöglichkeiten umsehen, die von Absentismus bis hin zu einem endgültigen Ausscheiden aus dem Betrieb führen können (vgl. Matiaske/Mellewigt 2001: 11). Auch dieses Modell ist nicht frei von Kritik, wie bspw., dass der Begriff des Anspruchsniveaus nicht ausreichend bestimmt wird bzw. die Frage danach, wie die Individuen den Vergleich zwischen Arbeitssituation und Erwartungen kognitiv vornehmen (vgl. Baumgartner/Udris 2006: 116). Dennoch scheinen gerade die verschiedenen Formen von Zufriedenheit der wesentliche Erkenntniszugewinn des Modells zu sein, da damit auch ,... ein relevanter Beitrag zur Klärung der befremdenden Ergebnisse, wonach sich unabhängig von Betrieb und Arbeit der Großteil der Befragten als zufrieden beschreibt, geleistet wird.“ (Kirchler/Hölzl 2008: 252) Betrachtet man die Studie des der Diskussion zu Grunde liegenden Qualitätsindizientableaus aus dieser Perspektive, so könnte derart durchaus auch der Zufriedenheitswert der Praktikanten argumentiert werden. Von den rund 1.400 Absolventen eines Praktikums gaben annährend $90 \%$ an, dass sie den Praktikumsbetrieb und somit auch den entsprechenden Betrieb weiterempfehlen würden (vgl. Ammann/Thoma 2011). Dieser hohe Zustimmungsgrad kann vor dem entfalteten theoretischen Hintergrund allerdings auch kritisch betrachtet werden.

Der Kritik an der Anreiz-Beitrags-Theorie bzw. am Bruggemann-Modell folgend kann die Frage aufgeworfen werden, inwieweit Praktikanten in der 
Lage sind Erwartungshaltungen bezogen auf ein Arbeitsfeld zu entfalten und insbesondere auch den komplexen kognitiven Prozess des Vergleichs $\mathrm{zu}$ vollziehen (vgl. Kirchler/Hölzl 2008: 252). Anders gewendet müsste die Frage konsequenterweise lauten: Wie hoch ist einerseits das Anspruchsniveau, welches Praktikanten an einen Praktikumsarbeitsplatz oder generell an das Praktikum haben und wie wird dieses andererseits von den Praktikanten beschrieben und insbesondere wie verläuft der Prozess des Vergleichs?

\section{Zwischenfazit}

Zusammenfassend scheinen beide Perspektiven einige interessante Implikationen zur qualitätsvollen Ausgestaltung von Betriebspraktik zu liefern. Aus der Herzbergschen Betrachtungsweise sind m. E. insbesondere die Hinweise, dass für die Tätigkeit in Betrieben klare Ziele notwendig sind, die Aufgaben und Tätigkeiten derart strukturiert werden, dass die Praktikantinnen das Gefühl gewinnen, weder unter- noch überfordert zu sein und, dass diese Verantwortung tragen dürfen, sehr interessant. „The factor that lead to positive job attitudes do so because they satisfy the indiviudal's need for selfactualization in his work. The concept of self-actualization, as a man's ultimate goal has been focal to the thought of many personality theorists." (Herzberg et al. 1959: 114) Analog zu regulären Arbeitsver-hältnissen könnte hier die These formuliert werden, dass die Präsenz bestimmter grundlegender Hygienefaktoren auch im Rahmen von Betriebspraktika sinnvoll und gewinnbringend erscheint und diese durchaus auch mit einem klassischen Arbeitsverhältnis zumindest verglichen werden können. Darüber hinaus berücksichtig die Herzbergsche Perspektive allerdings - verständlicherweise - bspw. Fragen der Begleitung des Praktikums durch einen Ansprechpartner oder die Vor- und Nachbereitung in der Schule nicht, wenngleich diese m. E. ebenso von Wichtigkeit sind. Hier müsste zumindest überlegt werden, wie die Spezifika eines Betriebspraktikums zugeordnet werden können. Aber auch die Motivatoren sind für das Praktikum zentral und dürfen nicht außen vor gelassen werden. Im Praktikum gilt, ebenso wie im regulären Arbeitsverhältnis, dass die Tätigkeit sehr zentral ist. In Summe soll somit die Tätigkeit aus der Perspektive der Praktikanten als gehaltvoll und sinnbringend empfunden werden, dann kann das Praktikum auch aus deren Perspektive als zufriedenstellend eingeordnet werden.

Insbesondere bezogen auf die Frage nach der Einordung von ,Zufriedenheit', sprich dem Zufriedenheitszustand, könnte mittels des Bruggemannschen Zugangs ein weiterer Blickwinkel geöffnet werden. Die verschiedenen ,Zufriedenheitszustände“ bieten eine gute Basis für eine weiterführende Analyse von Praktikumsarbeitsplätzen, speziell vor dem Hintergrund des hohen 
Zustimmungsgrades, den Praktikantinnen bei der Weiterempfehlung des Platzes einnehmen. Eine sorgfältige Ausdifferenz-ierung und Artikulation der Erwartungshaltungen auf der einen Seite und des ,Erhaltenen“ bzw. des ,Angebots' der Betriebe auf der anderen Seite, kann einen ersten Schritt in diese Richtung darstellen. Dieser Ansatz deckt sich auch mit den Aussagen - aus der Perspektive der Praktikumsbetreuer -, denen zufolge die Praktikanten nicht in der Lage sind, die Ziele, die sie im entsprechenden Praktikum erreichen sollen auszuformulieren. Dies kann verschiedene Ursachen haben, die nicht ausschließlich in der Vorbereitung des Praktikums in der Schule zu suchen sind sondern durchaus auch bei den Praktikanten selber, die schlicht nichts mit dem Praktikum verbinden. Ausgehend von diesen Überlegungen bestünde dann auch die Möglichkeit die verschieden Zufriedenheitsgrade und -zustände bzw. Handlungsstrategien der Praktikanten zu erforschen. Insbesondere die dabei zum Einsatz kommende Methode der ,strukturierten Tagebuchforschung' (Kirchler/Hölzl 2008: 246) könnte genutzt werden, um zu identifizieren, in welchen Momenten besonders unzufriedene Ereignisse auftreten und weshalb. Diese Ergebnisse würden dann wiederum zur Bestätigung bzw. Überarbeitung der verschiedenen Qualitätskriterien beitragen.

\section{Ausblick: Chancen und Grenzen der Perspektive}

Die eingenommene Perspektive der Arbeitszufriedenheit bietet m. E. diverse Chancen zur Formulierung eines Qualitätsmodells für Betriebspraktika, hat aber auch gewisse Grenzen, die berücksichtigt werden müssen. Die im Zwischenfazit angedeutete Methode zeigt auch schon einen möglichen Weg auf, der eingeschlagen werden kann, um sich einer weiteren Fundierung anzunähern.

Durchaus selbstkritisch muss an dieser Stelle - nebst der Tatsache, dass kein Forscher unvoreingenommen ins Feld geht und somit, das Denken und forschende Handeln schon vorstrukturiert ist - die Frage zulässig sein, weshalb die diskutierten theoretischen Perspektiven der Arbeitszufriedenheitsforschung überraschend viele Schnittmengen mit Forschungsarbeiten zur qualitätsvollen Ausgestaltung von Betriebspraktika aufweist. Funktioniert dieser Vergleich unter Umständen nur deshalb, weil betriebliche Praxisphasen mehr als Arbeitsverhältnisse, denn als Lern- UND Arbeitsverhältnisse gelebt werden? Damit soll keineswegs gesagt werden, dass ,Arbeiten' nichts mit ,Lernen' zu tun hat und ein Praktikant beim Übernehmen und Ausführen von Tätigkeiten nicht lernt. Es geht hier vielmehr um eine analytische Trennung, der didaktisch strukturierte und gestaltete Lernanlässe - bspw. das Übernehmen von Erkundungsaufträgen oder das Führen eines Interviews mit einem Vorgesetzten - zu Grunde gelegt werden, die dann auch in der Schule 
oder im Betrieb aufgearbeitet werden. Wenn dem so ist, dass derartige didaktische Überlegungen nicht oder nur in geringem Maße stattfinden, dann muss konsequenterweise die Frage gestellt werden, welche Auswirkungen dieses Verständnis der Organisation eines Praktikums auf das ,Lernen' in diesem hat oder ob diesem schon möglicherweise mit der Erweiterung von Handlungskompetenz durch das vermeintlich ,schlichte " Einbinden in die alltäglichen Tätigkeiten und somit die Wertschöpfungskette des Betriebes genüge getan wird. Derartige ,Zufriedenheit' mit diversen Situationen an den Arbeitsplätzen wird aus der Perspektive der Arbeitszufriedenheit auch häufig aus lerntheoretischer Sicht begründet, in dem darauf verwiesen wird, dass es sich hierbei um Konditionierungsprozesse handelt. Demzufolge kann Arbeit selbst, aber auch der Arbeitsplatz, mit seiner Ausstattung und den Menschen zunächst als neutraler Reize betrachtet werden. Entstehen im Zuge des Ausübens der Arbeit positive Gefühle und negative bleiben aus, so wird auch der Arbeitsplatz und die Zufriedenheit mit diesem und somit der Tätigkeit positiv konditioniert und erlangt Belohnungswert (Kirchler/Hölzl 2008: 245), was wiederum die Konsequenz mit sich bringt, dass Zufriedenheit entsteht oder gar steigt.

Wenn nun gegebenenfalls in einem Praktikum ,Arbeiten' das ausschließliche Kriterium darstellt und auf didaktisch strukturiertes, Lernen“ kein oder wenig Wert gelegt wird, so ist es nicht weiter erstaunlich, dass eben - wie oben angeführt - der Großteil der Absolventen mit dem Praktikum einen hohen Zufriedenheitsgrad verbinden. Erwartungshaltungen, die nicht vorhanden sind bzw. von der Schule oder Betreuern nicht kommuniziert werden, können auch nicht erfüllt werden und somit in diesem Verständnis auch nicht zu Unzufriedenheit führen. Wenn dem so ist und dies müsste $\mathrm{m}$. E. weiterführenden Überlegungen und Analysen zugeführt werden, dann sollte zumindest darüber nachgedacht werden, ob es diesbezüglich Verbesserungspotential gibt. Liegt dies bspw. in der Vorbereitung der Praktika in der Schule, in der Begleitung dieser oder schlicht in der Umsetzung, was wiederum Hinweise auf die in einem Qualitätsmodell zu formulierenden Kriterien bzw. Grundelemente schließen lässt. Eines lässt sich mit der Perspektive diverser Forschungsansätze zur Arbeitszufriedenheit zunächst keineswegs argumentieren und auch nicht analysieren: Die Frage ob und inwieweit in derartigen zeitlich befristeten Phasen ,Lernen' stattfindet oder welche Wirkungen diese auf selbiges haben könnten. Dies ist mit Sicherheit eine einzugestehende Limitation der vorgeschlagenen Blickrichtung.

Dennoch eignet sich die Perspektive - unter den gegebenen Einschränkungen - m. E. sehr gut, das Verständnis eines qualitätsvollen Praktikumsarbeitsplatzes zu schärfen und dahingehende Überlegungen zu fundieren. Speziell die Betrachtung von Arbeit als komplexes von verschiedenen Elementen abhängiges Ereignis und der damit einhergehenden Betrachtung von Arbeitsplätzen birgt m. E. einiges an Potential. So ist für ein erfolgreiches 
Praktikum eben nicht nur das Übernehmen von Tätigkeiten und Ausführen dieser durch das Individuum von Relevanz, sondern auch andere Elemente man denke hier nur an die Motivatoren und Hygienefaktoren nach Herzberg - könnten maßgeblichen Einfluss auf ein erfolgreiches Betriebspraktikum haben. Der Praktikant und damit die didaktische Begleitung seines, Lernens UND Arbeitens' werden somit nicht als theoretisches Konstrukt im luftleeren Raum konzipiert, welches losgelöst von der betrieblichen Realität agiert, sondern vielmehr als ein von einem Umfeld, von Strukturen, von Mitarbeiterinnen, Aufgaben usw. abhängiges und geprägtes temporäres Organisationsmitglied. Insbesondere die Qualitäts-indizien können vor diesem Hintergrund einer weiterführenden Analyse unterzogen werden, indem bspw. auf ausgewählte Erhebungsinstrumente der dargelegten Perspektive zurückgegriffen wird.

Dies könnte u. U. in der Adaptierung und Verwendung eines in der Arbeits- und Organisationspsychologie verwendeten standardisierten Instrumentes - wie bspw. der ,Skala zur Messung der Arbeitszufriedenheit' (SAZ) (Fischer/Lück 1972) oder dem ,Arbeitsbeschreibungsbogen“ (AAB) (Neuberger/Allerbeck 1978) - geschehen, um daraus neue und weiter-führende Erkenntnisse zu erlangen.

\section{Literatur}

Alt, R.: Mikropolitik. In: Weik, E./Lang, R.: Moderne Organisationstheorien, Eine sozialwissenschaftliche Einführung, S. 286-318, Wiesbaden.

Ammann, M./Thoma, M. (2011): Entwicklung eines Qualitätsmodells für Betriebspraktika. Erste empirische Einblicke. In: wissenplus, Sonderausgabe Wissenschaft, in press, Wien.

Ammann, M./Waltl, M. (2010): Wissenstransfer im Betriebspraktikum. Eine Betrachtung aus der Perspektive des „Cross-Border-Learning“ In: wissenplus, Sonderausgabe Wissenschaft, Heft 5-09/10, S. 16-19, Wien.

Barnard, Chester I. (1968): The Functions of the Executive, Cambridge, Mass., USA.

Baumgartner, C./Udris, I. (2006): Das „Zürcher Modell“" der Arbeitszufriedenheit 30 Jahre „still going strong“. In: Fischer, L. (Hrsg.): Arbeitszufriedenheit, S. 111-134, Göttingen.

van Buer, J./Troitschanskaja (2002): Das Betriebspraktikum als Lernort im Lernortverbund - zwischen Anspruch und Wirklichkeit. In: Berufs- und Wirtschaftspädagogik - online, Ausgabe 3, 1-17. Online: http://www.bwpat.de/ ausgabe3 /buer_troitschanskaja_bwpat3.pdf, Abgerufen am 20/10/10.

van Buer, J./Troitschanskaja, O. /Höppner, Y. (2004): Das Praktikum in der dreijährigen Berufsfachschule - Lernortkooperation oder Lernortkoordination? In: Euler, D. (Hrsg.): Handbuch der Lernortkooperation, S. 428-445, Bd. 1. Bielefeld. 
Engeström, Y. (2004): Activity theory and individual and social transformation. In: Engeström, Y./ Miettinen, R./Punamäki, R.-L. (Hrsg.): Perspectives on activity theory, S. 19-38, Cambridge University Press.

Fischer, L./Lück H. E. (1972): Entwicklung einer Skala zur Messung von Arbeitszufriedenheit (SAZ). In: Psychologie und Praxis, 16, S. 64-76.

Heffeter, B. (2008): Arbeitsmarktrelevanz der Ausbildung an österreichischen Handelsschulen. http://www.heffeter.com/cmsx/images/content/Endbericht\%20HAS.pdf, Abgerufen am 20/10/10.

Herzberg, Frederick et al. (1959): The motivation to work, Wiley, New York, USA.

Kirchler, E./Hölzl, E. (2008): Arbeitsgestaltung. In: Kirchler, E. (Hrsg.): Arbeits- und Organisationspsychologie, S. 199-316, Wien.

Kremer, H.-H./Gockel, C. (2010): Schülerbetriebspraktikum im Übergangssystem Relevanz, Potenziale und Gestaltungsanforderungen. In: Berufs- und Wirtschaftspädagogik-online, Ausgabe 17, 1-29. Online: http://www.bwpat.de/ ausgabe17/kremer_gockel_bwpat17.pdf, Abgerufen am 14/03/2010.

March, J. G./Simon, H. A. (1958): Organizations, New York, London, Sydney.

Matiaske, W./Mellewigt, T. (2001): Arbeitszufriedenheit: Quo vadis? Eine Längsschnittsuntersuchung zu Determinanten und Dynamik von Arbeitszufriedenheit. In: Die Betriebswirtschaft, 61. Jg., Nr. 1, S. 7-24.

Mayring, P. (2000): Qualitative Inhaltsanalyse. Grundlagen und Techniken, Weinheim und Basel.

Marcus, B. (2011): Einführung in die Arbeit- und Organisationspsychologie, Wiesbaden.

Nerdinger, F. W. (2011): Arbeitsmotivation und Arbeitszufriedenheit. In: Nerdinger, F. W./Blickle G./Schaper, N. (Hrsg.): Arbeits- und Organisationspsychologie, S. 393-408, Heidelberg.

Neuberger, O./Allerbeck, M. (1978): Messung und Analyse der Arbeitszufriedenheit: Erfahrungen mit dem „Arbeitsbeschreibungsbogen (AAB)“, Bern, Wien.

von Rosenstiel, L. von (2003): Arbeitszufriedenheit. In: von Rosenstiel, L. /Regnet, E./Domsch, M. (Hrsg.): Führung von Mitarbeitern, Handbuch für erfolgreiches Personalmanagement, S. 195-216, Stuttgart.

von Rosenstiel, L. (2007): Grundlagen der Organisationspsychologie, Basiswissen und Anwendungshinweise, Stuttgart.

Ostendorf, A. (2007): Das Betriebspraktikum zwischen fruchtbarer Lernsituation und Disziplinierungstechnologie. In: Münk, D. et al. (Hrsg.): Hundert Jahre kaufmännische Ausbildung in Berlin, S. 164-173, Opladen.

Ostendorf; A. (2008): Betriebspraktikum als Lernsituationen gestalten. In: Wissenplus, Sonderausgabe Wissenschaft, Heft 5-07/08, S. 18-22, Wien.

Stahl, H. K. (2003): Voraussetzungen für ein nachhaltig gelungenes StakeholderManagement. In: Matzler, K./Pechlaner, H./Renzl, B. (Hrsg.): Werte schaffen. Perspektiven einer stakeholderorientierten Unternehmensführung, S. 21-47, Wiesbaden.

Weinert A. B. (2004): Organisations- und Personalpsychologie, Weinheim und Basel. 



\title{
Digitale Technologien als Tools zur Förderung der Konnektivität des Lernens in Schule und Betrieb
}

\author{
Carmela Aprea, Christoph Arn, Elena Boldrini, Alberto Cattaneo, \\ Elisa Motta, Alicja Sroka
}

\section{Einleitung}

Von dualen Berufsbildungssystemen wird erwartet, dass durch das Alternieren schulischer und betrieblicher Phasen sowohl der für die effektive Bewältigung beruflicher Arbeitsanforderungen erforderliche Wissensaufbau als auch die Motivation der Auszubildenden wirksam unterstützt und damit optimale Bedingungen für die integrative Entwicklung beruflicher Handlungskompetenz und Identität geschaffen werden können (z.B. Tenberg 2011). Wie sich in empirischen Untersuchungen der berufsbezogenen LehrLernforschung zeigt, gelingt es indes oftmals nicht im erforderlichen Ausmaß, bei den Lernenden jene kognitiven und metakognitiven Prozesse anzuregen, mit deren Hilfe betriebliche Erfahrungen und schulische Lerninhalte angemessen miteinander verknüpft werden können (z.B. Ostenk 2009; Zinn 2010). Ebenso finden sich immer wieder Befunde, die deutliche Unterschiede in Bezug auf Interesse und Motivation in Schulen und Ausbildungsbetrieben konstatieren, wobei die Auszubildenden dem betrieblichen Lernen generell höhere Relevanz beimessen (z.B. Volet \& Järvela 2001; Rosendahl \& Straka 2007). Diese Befunde, die auch in Gesprächen mit Berufsbildungsverantwortlichen und Auszubildenden eindrücklich bestätigt werden (z.B. Dillenbourg et al. 2010), werfen neben dem Erfordernis einer verbesserten institutionellen und curricularen Abstimmung der Lernorte auch die Frage auf, wie berufliches Lernen instruktional so gestaltet werden kann, dass die Verbindung zwischen den Lernorten für die Auszubildenden deutlicher sichtund erfahrbar wird, und eine integrative Kompetenzentwicklung damit effektiv unterstützt werden kann.

Im vorliegenden Beitrag wird über ein vom Schweizerischen Bundesamt für Berufsbildung und Technologie (BBT) als Leading House gefördertes Forschungsprogramm berichtet, welches diese Frage explizit aufgreift. Die Forschungsaktivitäten sind insbesondere daraufhin orientiert, zu prüfen, ob und inwieweit digitale Technologien dabei helfen können, die Kluft zwischen den Lernorten zu überbrücken. Der Beitrag ist wie folgt aufgebaut: Um eine Einordnung der Überlegungen zu ermöglichen, wird im zweiten Abschnitt zunächst ein Überblick über das gesamte Forschungsprogramm des Leading 
Houses „Technologien für die Berufsbildung: Dual-T“ gegeben. Im Abschnitt 3 wird sodann der konzeptuelle Rahmen des Programms skizziert, während im Abschnitt 4 exemplarisch eine empirische Studie zur Nutzung von videobasierten Lernmaterialien in der beruflichen Erstausbildung von angehenden Automobil-Mechatroniker/-innen vorgestellt wird. Die Ausführungen enden mit einem kurzen Fazit und Ausblick auf die in Aussicht genommenen weiterführenden Forschungsarbeiten.

\section{Das Leading House „Technologien für die Berufsbildung“" im Überblick}

Das Leading House „Technologien für die Berufsbildung“ ist seit 2006 aktiv. In der gegenwärtigen zweiten Projektphase, deren Förderung im Oktober 2009 begann, sind vier Schweizerische Forschungsinstitutionen beteiligt. ${ }^{1}$ Die Forschungsarbeiten orientieren sich an dem im nächsten Abschnitt näher zu erläuternden Konzept des „Erfahrraums“ und umfassen momentan drei Projektgruppen, die jeweils eine spezifische Technologie bzw. damit verbundene Repräsentationsformen (d.h. graphische, sprachliche, gegenständliche) in den Blick nehmen. In diesen Projektgruppen wurden bislang insgesamt 16 empirische Studien durchgeführt, in die mehr als 1.300 Auszubildende sowie rund 150 Berufsbildungsverantwortliche aus allen Schweizerischen Sprachregionen und sechs verschiedenen beruflichen Domänen einbezogen waren. Die drei Projektgruppen lassen sich wie folgt skizzieren:

1 Wissenskonstruktion und -elaboration durch Nutzung visueller Medien: In diesem Projekt werden mit Hilfe von mobilen Technologien vorrangig visuelle Werkzeuge entwickelt und eingesetzt, mit denen Erfahrungen am Arbeitsplatz festgehalten und im berufsschulischen Unterricht eingebracht und elaboriert werden können. Beispielsweise werden betriebliche Abläufe per Mobiltelefon fotografiert oder mit Hilfe von HeadbandKameras auf Video aufgenommen und im Unterricht reflektiert. Ferner werden Technologien auch dafür genutzt, Fragen und Unterstützungsbe-

1 Diese Institutionen sind: Das Centre de Recherche et d'Appui pour la Formation et ses Technologies (CRAFT) der Ecole Polytechnique Fédérale de Lausanne, die Einheit Technologies de Formation et d'Apprentissage (TECFA) an der Fakultät für Psychologie und Erziehungswissenschaften der Universität Genf, das Departement für Erziehungswissenschaften der Universität Fribourg sowie das Team „Innovationen“ des Forschungsschwerpunkts „Aktuelle Kontexte der Berufsbildung“ am Eidgenössischen Hochschulinstitut für Berufsbildung (EHB). 
dürfnisse der Lernenden zeitnah zu ihrem Auftreten individuell zu bearbeiten. $^{2}$

2 Kollaboratives computergestütztes Schreiben zur Reflexion betrieblicher Abläufe: In Zusammenarbeit mit Lehrkräften an Berufsschulen werden in diesem Projekt Aktivitäten des kollaborativen Schreibens entwickelt, welche die Lernenden darin unterstützen sollen, ihre beruflichen Erfahrungen zu dokumentieren, auszutauschen und vor allem zu reflektieren. Genutzt werden hierbei insbesondere Web 2.0 Technologien (Blogs, Wikis), die durch weitere visuelle Medien (Videos, Bilder etc.) ergänzt werden können. ${ }^{3}$

3 Problemlösen durch den Einsatz von „,Tangibles “: Das Projekt untersucht das Potenzial von Tangible User Interfaces (physische Objekte, die zur Steuerung und zur Interaktion mit dem Computer genutzt werden), um das Lernen und Verstehen von komplexen Entscheidungsprozessen und theoretischen Zusammenhängen zu unterstützen. Eigens für das Projekt entwickelte Simulationstools (so genannte Tinker-Lamps, mit denen sich z.B. ein Lager für Waren in verkleinertem Maßstab einrichten lässt sowie deren $\mathrm{Zu}$ - und Abgang physisch nachvollzogen werden kann) werden dafür eingesetzt, den Lernenden solche Arbeitsprozesse zu veranschaulichen, mit denen sie in ihrer Ausbildungspraxis gar nicht oder nicht in hinreichendem Umfang konfrontiert werden, die aber dennoch für ein Durchdringen der Domäne von zentraler Bedeutung sind. ${ }^{4}$

Wie im folgenden Abschnitt weiter ausgeführt werden soll, besteht die Besonderheit aller im Forschungsprogramm Dual-T angesiedelten Projekte darin, dass digitale Technologien hier nicht primär als Medien zur elektronischen Speicherung von Lernmaterialien eingesetzt werden, sondern vorrangig der Evokation von Lernaktivitäten zur Verknüpfung von Erfahrungen und Wissensbeständen aus verschiedenen Lernorten dienen.

2 In die empirischen Studien der ersten Projektgruppe wurden bislang die Ausbildungsberufe „Automobil-Mechatroniker/-in“, „Bäcker/-in, Konditor/-in, Confiseur/-in“ sowie „,Koch/ Köchin“ einbezogen. Für weitere Informationen zu den Studien dieser Projektgruppe vgl. z.B. Gurtner et al. 2011 sowie Dillenbourg et al. 2010.

3 Die empirischen Studien der zweiten Projektgruppe wurden bislang mit angehenden Dentalassistenten/-assistentinnen, Kaufleuten sowie Fachmänner/-frauen Gesundheit durchgeführt. Für weitere Informationen zu den Studien dieser Projektgruppe vgl. z.B. Dillenbourg et al. 2010 sowie Gavota et al. 2010.

4 An den empirischen Studien der dritten Projektgruppe waren bislang Zimmerleute und Logistiker/-innen beteiligt. Für weitere Informationen zu den Studien dieser Projektgruppe vgl. z.B. Arn \& Sroka 2011 sowie Dillenbourg et al. 2010. 


\section{Konzeptueller Rahmen: Der „Erfahrraum“}

Wie bereits angedeutet, wurde zur Fundierung des Forschungsprogramms im Leading House „Technologien für die Berufsbildung“ das Konzept des „Erfahrraums" entwickelt. Dieses Konzept ist unter anderem durch pragmatische und kulturhistorische Rahmentheorien sensu Dewey (1997) und Vygotsky (1962) inspiriert. Ferner greift es auf Ansätze des „Computer-Supported Collaborative Learning (CSCL)“ (z.B. Dillenbourg \& Fischer 2007) und der „Human-Computer Interaction (HCI)“(z.B. Dix et al. 2004) zurück. Der Zusammensetzung des Neologismus“ „Erfahrraum“" entsprechend wird berufliches Lernen in diesem Konzept als ein zirkulärer Prozess des Navigierens in einem virtuellen, über die Lernorte Betrieb und Schule hinweg verlaufenden Feld konzipiert (vgl. Abbildung 1).

\section{Abbildung 1: Der Erfahrraum: Aktivitäten im Rahmen des kontextübergreifenden beruflichen Lernens}

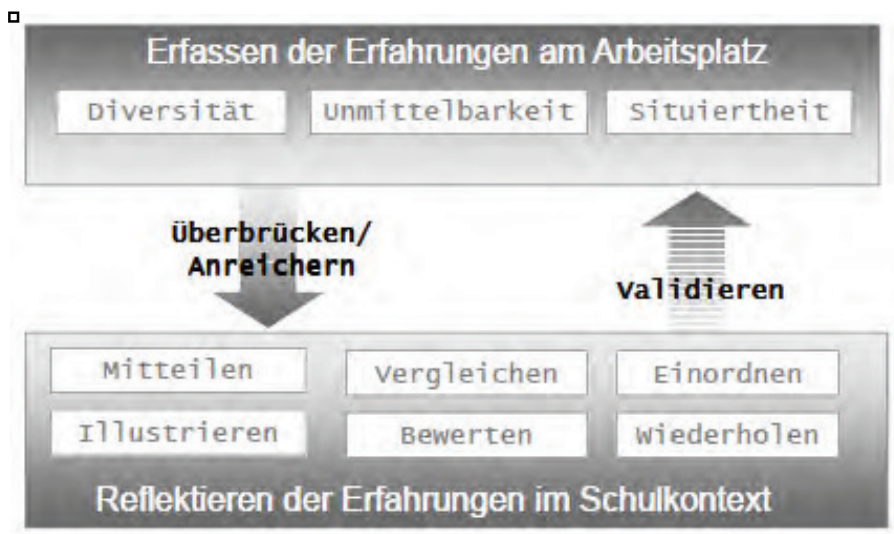

Der Prozess erstreckt sich über die beiden Phasen des Erfassens von Erfahrungen am Arbeitsplatz und deren Reflexion im Schulkontext sowie mehrere darin eingeschlossene Lernaktivitäten, die durch moderne Lerntechnologien unterstützt werden können und sich wie folgt spezifizieren lassen:

5 Auf die für den deutschen Sprachgebrauch eher typische Substantivierung („Erfahrungsraum“) wurde bewusst verzichtet, um durch den Bezug zum Verb „Erfahren“ den Tätigkeits- bzw. Aktivitätscharakter zu unterstreichen. 
1 In der ersten Phase geht es zunächst darum, die Erfahrungen der Lernenden am Arbeitsplatz festzuhalten. Dies sollte optimaler Weise so geschehen, dass das ganze Spektrum an unterschiedlichen Nuancierungen einer spezifischen beruflichen Tätigkeit abgebildet werden kann (z.B. Lagerhaltung bei verschiedenen Arten von Gütern wie etwa Bücher vs. Frischwaren). Da zeitlich nachgelagerte Rekonstruktionen betrieblicher Erfahrungen für die Lernenden eher schwierig zu bewältigen sind, sollte es durch den Technologieeinsatz ferner möglich werden, die Erfahrungen unmittelbar im Moment ihres Entstehens zu erfassen. Ebenso sollten alle relevanten Aspekte einer bestimmten beruflichen Situation (z.B. verwendete Materialien und Werkzeuge, Interaktionen mit Kolleginnen und Kollegen) abgebildet werden können. Die auf diese Art und Weise gewonnenen visuellen, textlichen und gegenständlichen Repräsentationen beruflicher Erfahrungen bilden eine Brücke zum Schulkontext, wo sie - gegebenenfalls mit ergänzenden didaktischen Elementen angereichert - einer weiteren Elaboration zugänglich gemacht werden können.

2 Der Aufbau von fundiertem beruflichen Wissen und Können geschieht nicht durch bloße Erfahrung alleine, sondern erfordert deren Reflexion und Elaboration. Lerntechnologien können in dieser Hinsicht im Schulkontext beispielsweise dazu dienen,

- die in der ersten Phase festgehaltenen Erfahrungen mit den anderen Lernenden zu teilen und durch weitere Beispiele zu illustrieren.

- Gemeinsamkeiten und Unterschiede in der Tätigkeitsausführung zu vergleichen und einer kritischen Würdigung zu unterziehen.

- die Lerninhalte in den größeren Kontext der Wissensdomäne einzuordnen und - was insbesondere im Falle von algorithmischen Prozeduren von zentraler Bedeutung ist - diese Lerninhalte $\mathrm{zu}$ wiederholen.

3 Die Lernaktivitäten im Schulkontext sollen vor allem die schrittweise Dekontextualisierung bzw. Generalisierung (bzw. gegebenenfalls auch Diskriminierung sensu Anderson (z.B. 1993)) des angeeigneten Wissens und Könnens unterstützen. Diese wiederum sollten in einem anschließenden Schritt in den Arbeitskontext rücküberführt und dort valdiert sowie weiter verfeinert werden.

Wie im folgenden Abschnitt am Beispiel der Nutzung von videobasierten Lernmaterialien noch detaillierter gezeigt werden soll, gehen wir davon aus, dass sich die unterstützenden Effekte von Lerntechnologien nicht als Selbstläufer ergeben, sondern - wie in jedem anderen Lehr-Lernarrangement - einer auf die Lerngruppe, die Lerninhalte und weitere didaktische Rahmen- 
bedingung abgestimmten Gestaltung bedürfen (in Bezug auf den Einsatz von Lerntechnologien vgl. z.B. Dillenbourg \& Jermann 2010; allgemein zur funktionalen Gestaltung beruflicher Lehr-Lernarrangements vgl. z.B. Aprea/ Ebner/Müller 2010).

\section{Exemplarische Studie zur Nutzung von videobasiertem Lernmaterial in der beruflichen Erstausbildung}

Um zu verdeutlichen, wie sich das „Erfahrraum“-Konzept im Kontext der beruflichen Erstausbildung konkretisieren lässt, wird in diesem Abschnitt über die theoretischen Grundannahmen, das methodische Vorgehen und ausgewählte Ergebnisse einer quasi-experimentellen Feldstudie berichtet. Ziel dieser Studie war es, einen ersten Beitrag zur Beantwortung der Frage zu leisten, inwiefern Videoaufnahmen von betrieblichen Tätigkeiten als Erfahrungen der Lernenden effektiv im berufsschulischen Unterricht eingesetzt werden können. An der Studie nahmen 59 Schüler und eine Schülerin des vierjährigen Ausbildungsberufs „Automobil-Mechatroniker/-in mit eidgenössischem Fähigkeitszeugnis (EFZ)“ aus drei Klassen einer Tessiner Berufsschule teil. Zum Zeitpunkt der Durchführung der Studie befanden sich die Lernenden im zweiten Ausbildungsjahr und ihr Durchschnittsalter betrug 18 Jahre (Min = 16; Max =32). Die Lernenden in den drei Klassen unterschieden sich nicht im Hinblick auf die Verteilung des mittels der Vorjahresnote im berufskundlichen Unterricht erhobenen domänenspezifischen Wissens und Könnens. Neben den Schülerinnen und Schülern waren außerdem die Fachlehrenden der drei Klassen sowie weitere 12 Lehrkräfte und betriebliche Ausbildungsverantwortliche in die Studie involviert.

\subsection{Theoretische Grundannahmen}

Als weitere Spezifikationen des „Erfahrraum“-Konzepts liegen der Studie zur Nutzung von videobasiertem Lernmaterial in der beruflichen Erstausbildung die folgenden Annahmen zugrunde:

- In Übereinstimmung mit aktuellen Ansätzen aus der Pädagogischen Psychologie (z.B. Klauer \& Leutner 2007) ebenso wie aus der berufs- und wirtschaftspädagogischen Lehr-Lernforschung (z.B. Nickolaus \& Pätzold 2011 sowie Seifried et al. 2010) fassen wir Lernen als einen vor dem Hintergrund individueller Lernvoraussetzungen (z.B. Vorwissen, Interessen, Überzeugungen) sowie in Auseinandersetzung mit der sozialen und gegenständlichen Lernumgebung erfolgenden Prozess der Kon- 
struktion von Wissen auf, der auf die Ausbildung von für die Bewältigung beruflicher Anforderungen geeigneter mentaler Repräsentationen gerichtet ist.

- In Anlehnung an Theorien des multimedialen Lernens (z.B. Mayer 2008) nehmen wir zudem an, dass dieser Prozess auf der aktiven Verarbeitung von Information basiert und die folgenden Speicher und Sub-Prozesse umfasst:

- Externe Information (z.B. in Form von schriftlichen oder mündlichen Texten, statischen oder dynamischen Visualisierungen) wird als Stimulus über die Sinnesorgane im sensorischen Speicher aufgenommen. Über Prozesse der Aufmerksamkeitssteuerung findet dann eine Selektion statt, d. h. es wird von dem oder der Lernenden entschieden, was als wichtig anzusehen ist und weiter verarbeitet werden soll. Nur die als relevant erachteten Informationselemente gelangen ins Kurzzeitgedächtnis.

- Um aus den Informationselementen im Kurzzeitgedächtnis neues Wissen - oder anders ausgedrückt: Bedeutung - zu konstruieren, müssen sie mit Elementen aus dem Vorwissen in Verbindung gebracht werden. Die im Langzeitgedächtnis gespeicherten Wissensbausteine werden abgerufen. Sie stellen die Basis zur Bearbeitung der neuen Information dar. Ferner werden sie durch die Verknüpfung mit der neuen Information auch selbst verändert. Neue und bereits vorhandene Wissenselemente müssen durch Organisationsprozesse zudem in eine sinnvolle Struktur (z. B. hierarchische Ordnung oder Kausalbeziehung) gebracht werden.

- Die so verarbeiteten Informationselemente werden im Langzeitgedächtnis integriert und gespeichert, wo sie als Grundlage für (sofortige oder spätere) Lern- und Handlungsprozesse zur Verfügung stehen.

- Des Weiteren folgen wir der Annahme, dass die Prozesse der Informationsverarbeitung (vor allem bei durch psycho-motorisches oder kommunikatives Handeln gekennzeichneten) betrieblichen Abläufen durch geeignetes Videomaterial wirksam unterstützt werden können. Gemäß der in diesem Kontext einschlägigen Forschungsliteratur (z.B. Schnotz \& Lowe 2008) sowie erster empirischer Befunde (z.B. Schwan \& Riempp 2004) sollten sich die positiven Effekte dieser Form der Informationsdarbietung insbesondere mit dem Einsatz von interaktiven Hypervideos realisieren lassen. Interaktive Videos bieten die Möglichkeit, das Abspielen zu starten, zu stoppen, bestimmte Stellen im Video anzuwählen, Standbilder zu erzeugen, vor- und zurückzuspulen sowie die Abspielgeschwindigkeit zu variieren, so dass die Lernenden weitgehende Kontrolle 
über den Lernprozess erhalten. Aufgrund der Gestaltung als Hypervideo sind darüber hinaus die folgenden Vorteile zu vermuten: Die Hyperlinks können dabei helfen, die Aufmerksamkeit der Lernenden auf relevante Aspekte der betrieblichen Abläufe zu lenken. Die durch diese Links induzierte Vernetzung mit zusätzlichen textlichen, bildlichen oder grafischen Informationen (so genannte multimediale Fußnoten) unterstützen die Integration des Wissens. Sie dienen als Anker, an dem betriebliche Erfahrungen und schulische Lerninhalte respektive prozedurale und konzeptuelle Wissenselemente gemeinsam ,andocken“ können. Durch weitere didaktische Anreicherungen wie z.B. Untergliederung der Abläufe, Demonstrationen von verschiedenen Vorgehensweisen sowie weiterführende Lern- und Übungsaufgaben wird ferner die Wissensorganisation sowie die Flexibilität des Wissens unterstützt und es werden Reflexionsprozesse angeregt.

- Schließlich gehen wir davon aus, dass sich das lernförderliche Potential von interaktiven Hypervideos ähnlich wie bei anderen intruktionalen Interventionen nur dann entfalten kann, wenn ihr Nutzen von den Lernenden auch wahrgenommen wird (vgl. z.B. Aebli \& Ruthemann 1987). Gleiches gilt für die Lehrenden, die durch ihr didaktisches Handeln die Möglichkeiten und Grenzen der Potentialentfaltung maßgeblich steuern. Dieser Überlegung entsprechend sind bei der Evaluation der Lernwirksamkeit von interaktiven Hypervideos neben den kognitiven Lernleistungen auch die auf die Lernförderlichkeit bezogenen Urteile der beteiligten Akteure einzubeziehen.

\subsection{Methodisches Vorgehen}

Der Ablauf und das Design der Studie sowie die verwendeten Materialien und Instrumente sahen folgendermaßen aus:

1 Bei der Planung des Schuljahres wurden von den in den drei Klassen unterrichtenden Lehrkräften auf Basis der Lehrpläne für den Ausbildungsberuf „Automobilmechatroniker/-in“ und in Absprache mit den Ausbildungsverantwortlichen in den Werkstätten zunächst exemplarisch vier Unterrichtseinheiten im Umfang von je einer doppelten Unterrichtsstunde ausgewählt, die als besonders relevant erachtete betriebliche Abläufe thematisieren. Diese Auswahl umfasste die folgenden Prozeduren: (a) Check und Wartung der Batterie, (b) Einstellung von XENONScheinwerfern, (c) Einstellung der Einspritzanlage, (d) Diagnose von Fehlfunktionen im Kühlsystem.

2 Zu Beginn des Schuljahrs wurden die Lernenden im Klassenverband einer von drei Versuchsgruppen zugewiesen, nämlich: Einer ersten Expe- 
rimentalgruppe, für die der Unterricht zu den ausgewählten Einheiten durch didaktisch erweiterte, interaktive Hypervideos unterstützt werden sollte (EXP-PLUS), einer weiteren Experimentalgruppe, bei denen Videos in ,Rohform', d.h. ohne weitere didaktische Aufbereitung vorgesehen waren (EXP-ROH) sowie eine Kontrollgruppe ohne videobasierte Lernmaterialien. Gemäß unseren theoretischen Annahmen (vgl. Abschnitt 4.1) erwarteten wir, dass die Lernenden der EXP-PLUS Gruppe im Vergleich zur EXP-ROH Gruppe signifikant bessere Lernleistungen erzielen, und letztgenannte Versuchspersonen wiederum besser abschneiden als jene der Kontrollgruppe. Ebenso sollten die Hypervideos im Vergleich zu den Videos in Rohform höhere Werte in Bezug auf die wahrgenommene Lern- und Lehrförderlichkeit aufweisen.

3 In Rücksprache mit den Ausbildungsbetrieben wurden die Lernenden der beiden Experimentalgruppen vor der Durchführung der Unterrichtseinheiten von den Lehrkräften damit beauftragt, die ausgewählten Prozeduren an ihren Arbeitsplätzen mit Hilfe von Headband-Kameras auf Video aufzunehmen. Aus diesen Aufnahmen trafen die Lehrkräfte in gemeinsamer Abstimmung eine Auswahl von jeweils einem Video pro Unterrichtseinheit. Für die EXP-PLUS Gruppe wurden auf dieser Basis und unter Berücksichtigung der im Abschnitt 4.1 ausgeführten Überlegungen vier interaktive Hypervideos angefertigt. $\mathrm{Zu}$ allen thematischen Einheiten wurden von den Lehrkräften zudem weitere Unterrichtsmaterialien (insbesondere Einstiegssequenzen, Lern- und Übungsaufgaben, ergänzende textliche und/oder grafische Verständnishilfen) zusammengestellt bzw. entwickelt. Die Unterrichtseinheiten wurden in den drei Klassen wie oben geschildert durchgeführt.

4 Im Abstand von einigen Tagen nach Durchführung der jeweiligen Unterrichtseinheit bearbeiteten die Lernenden einen Test, der auf das betreffende Themengebiet abgestimmt war. Die vier Lerntests (im Folgenden bezeichnet als LT1-4) umfassten zwischen 8 und 15 Testfragen und dauerten etwa 40 bis 50 Minuten. Mit den Testaufgaben wurde neben einschlägigem Faktenwissen (z.B. Bezeichnung von Bestandteilen der Einspritzanlage) vor allem prozedurales und konzeptuelles Wissen geprüft, das zur Erläuterung von Zusammenhängen, zur Begründung der Abläufe oder zur Vorhersage von Reaktionsweisen genutzt werden sollte. Darüber hinaus wurde am Ende des Schulhalbjahres ein Abschlusstest (im Folgenden bezeichnet als LT final) geschrieben, in dem die Lernenden strukturäquivalente Aufgaben zum gesamten Inhaltsgebiet bearbeiten sollten. Schließlich wurden die Lernenden gebeten, die von ihnen wahrgenommene Lernförderlichkeit auf einer sieben-stufigen LikertSkala einzuschätzen. Zu diesem Zweck wurde den Lernenden der Kontrollgruppe anhand eines Beispiels ein Hypervideo bzw. Video in 
Rohform und jenen der beiden Experimentalgruppe das jeweils in ihrer Gruppe nicht genutzte Video vorgestellt. In vergleichbarer Weise mit entsprechender Umformulierung der Frage in Bezug auf die Lehrperspektive wurde bei den beteiligten Berufsbildungsverantwortlichen vorgegangen. Alle erhobenen Daten $\mathrm{zu}$ den Lernleistungen und der wahrgenommenen Lern- bzw. Lehrförderlichkeit wurden deskriptiven und inferenzstatistischen Analysen (t-Tests, ANOVAs) unterzogen.

\subsection{Ausgewählte Ergebnisse}

In Bezug auf die Lernleistungen sind die Ergebnisse der eben genannten Analysen in Tabelle 1 dargestellt. Wie sich in den Daten dieser Tabelle zeigt, schneidet die Gruppe der Versuchspersonen, die mit interaktiven Hypervideos lernten mit Ausnahme des dritten Lerntests signifikant besser ab als jene der Versuchspersonen, die nur mit den Videos in Rohform oder gänzlich ohne Videounterstützung lernten.

Tabelle 1: Ergebnisse „Lernleistungen (\% richtige Lösungen)“

\begin{tabular}{|c|c|c|c|c|c|}
\hline & LT1 & LT2 & LT3 & LT4 & LT-final \\
\hline EXP-PLUS & $65.20 * 7$ & $\left.77.17)^{*}\right)^{*}$ & 54.39 & $\left.57.31)^{*}\right)^{*}$ & $65.500_{\star \star *}$ \\
\hline EXP-ROH & 38.90 & 59.53 & 53.83 & 45.97 & 53.13 \\
\hline KONTROLL & $47.40]$ & 59.86 & 57.76 & 52.57 & 56.34 \\
\hline$F$ & 26.D0 & 11.40 & 264 & 7.21 & 3.66 \\
\hline Dr & $(2 ; 53)$ & $(2 ; 53)$ & $(2 ; 50)$ & $(2 ; 47)$ & $(2 ; 50)$ \\
\hline \multicolumn{6}{|c|}{$p<.10$} \\
\hline
\end{tabular}

Die Effektstärken bewegen sich im mittleren Bereich (>.30) für den Lerntest 4 und für den Abschlusstest; ein starker Effekt (>.50) wird hingegen für die Lerntests 1 und 2 ausgewiesen. Eine im Vergleich zur Kontrollgruppe bessere Lernleistung der Gruppe mit Video in Rohform ist indes nicht zu verzeichnen, sondern es deutet sich im Gegenteil für den Lerntest 1 sogar eine tendenziell negative Wirkung an.

Wie Analysen auf der Ebene der Einzelitems (vgl. Cattaneo \& Nguyen 2011) zudem erkennen lassen, tritt eine statistisch signifikante Überlegenheit in Bezug auf die Lernleistungen der Gruppe mit interaktiven Hypervideos vor allem bei solchen Testaufgaben zutage, die explizit eine Verbindung von 
konkreten Vorstellungsleistungen mit konzeptuellen Erklärungsmustern (z.B. Vorhersage von Reaktionsweisen) erforderlich machen.

Die weitgehende Überlegenheit der Hypervideos spiegelt sich schließlich auch in den Wahrnehmungen der Lernenden wider, die signifikant höhere Einschätzungen der Lernförderlichkeit von Hypervideos ( $\mathrm{M}=6.39$; $\mathrm{SD}=.69)$ im Vergleich zu den Videos in Rohform $(\mathrm{M}=5.24 ; \mathrm{SD}=1.20)$ vornahmen $(p<.05)$. Gleiches trifft mit den Mittelwerten von $5.70(\mathrm{SD}=.86)$ für die Hypervideos bzw. 4.87 ( $\mathrm{SD}=.73$ ) für die Videos in Rohform in Bezug auf die von den Berufsbildungsverantwortlichen eingeschätzte Lehrförderlichkeit zu $(p<.01)$.

\section{Fazit und Ausblick}

Die im vorangegangenen Abschnitt dargestellten Befunde stützen die Vermutung, dass interaktive Hypervideos zumindest für einen Teil der hier ausgewählten Lerninhalte den Wissensaufbau in der beruflichen Erstausbildung von Automobilmechatroniker/-innen wirksam befördern und dabei helfen können, eine Brücke zwischen den beiden Lernorten zu schlagen. Lediglich im Fall des Lerntests 3 lässt sich eine statistisch signifikante Mittelwertdifferenz nicht nachweisen. Nach Ansicht der beteiligten Lehrkräfte ist dieses Ergebnis darauf zurückzuführen, dass die Lerninhalte dieses Lerntests mit Abstand am schwierigsten waren. Es ist daher möglich, dass hier ein Bodeneffekt induziert wurde. Wie in weiteren Untersuchungen zu eruieren sein wird, könnte eine Alternativerklärung für dieses Ergebnis jedoch auch in einer nicht ausreichend guten Designqualität des betreffenden Hypervideos begründet liegen. Zudem ist uns bewusst, dass neben dem kleinen Stichprobenumfang auch die im bisherigen Untersuchungsdesign aus Gründen der ökologischen Validität unterlassene Randomisierung der Versuchspersonen sowie ein möglicher Einfluss von Unterschieden bezüglich der Kompetenz und Persönlichkeitseigenschaften der drei Lehrpersonen zu einer vorsichtigen Interpretation der Ergebnisse gemahnen bzw. deren weitere empirische Absicherung nötig machen. Um Erklärungsansätze für die skizzierte Befundlage zu generieren, sind zudem weiterführende Analysen der mentalen Repräsentationen und Lernprozesse wünschenswert. Neben einer umfänglicheren Analyse der in Frage kommenden Lerninhalte und einer entsprechenden curricularen Integration von interaktiven Hypervideos werden wir in zukünftigen Untersuchungen ferner der Frage nachgehen, ob und inwieweit die Effektivität dieses Lernmediums gesteigert werden kann, wenn Lernende dazu aufgefordert werden, selbst Hypervideos zu erstellen. 


\section{Literatur}

Aebli, H. \& Ruthemann, U. (1987): Angewandte Metakognition: Schüler vom Nutzen der Problemlösestrategien überzeugen. In: Zeitschrift für Entwicklungspsychologie und Pädagogische Psychologie, 19(1), S. 46-64.

Anderson, J. R. (1993): Rules of the Mind. Hillsdale, NJ.

Aprea, C./Ebner, H. G./Müller, W. (2010): Ja mach nur einen Plan... - Entwicklung und Erprobung eines heuristischen Ansatzes zur Planung kompetenzorientierter wirtschaftsberuflicher Lehr-Lern-Arrangements. In: W\&E, 4, S. 91-99.

Arn, C. \& Sroka, A. (2011): Das Warenlager im Klassenzimmer. Folio (3), S. 39-43.

Cattaneo, A., \& Nguyen, A. T. (2011). Scuolavisione: OER and Hypervideos in the Swiss VET System. (Manuscript submitted to e-Learning and Digital Media).

Dewey, J. (1997): Experience and Education. (Originalausgabe 1938), New York.

Dillenbourg, P. \& Fischer, F. (2007): Basics of Computer-Supported Collaborative Learning. In: D. Euler (Hrsg.): Kooperatives Lernen in der beruflichen Bildung, ZBW Beiheft 21. Stuttgart, S. 111-130.

Dillenbourg, P. \& Jermann, P. (2010): Technology for Classroom Orchestration. In: M. S. Khine \& I. M. Saleh (Eds.): New Science of Learning: Cognition, Computers and Collaboration in Education. Dordrecht, pp. 525-552.

Dillenbourg, P./Bétrancourt, M./Gurtner, J.-L./Jermann, P./Schneider, D./Cattaneo, A. (2010): The Dual-T Leading House. Internal Working paper. Lausanne et al.

Dix, A./Finlay, J./Abowd, G. D./Beale, R. (2004): Human-Computer Interaction. New York.

Gavota, M./Cattaneo, A./Arn, C./Boldrini, E./Motta, E./Schneider, D. K./ Bétrancourt, M. (2010): Computer-Supported Peer Commenting: A Promising Instructional Method to Promote Skill Development in Vocational Education. In: Journal of Vocational Education \& Training, 62(4), pp. 495-511.

Gurtner, J.-L./Cattaneo, A./Motta, E./Mauroux, L. (2011): How Often and for What Purposes Apprentices Seek Help in Workplaces: A Mobile Technology-Assisted Study. In: Vocations and Learning, 4(2), pp. 113-131.

Klauer, K. J. \& Leutner, D. (2007): Lehren und Lernen. Weinheim.

Mayer, R. E. (2008): Learning and Instruction. $2^{\text {nd }}$ ed. Upper Saddle River.

Nickolaus, R. \& Pätzold, G. (2011): Lehr-Lernforschung in der gewerblichtechnischen Berufsbildung. ZBW Beiheft 25. Stuttgart.

Ostenk, J. (2009): Connections of School- and Workbased Learning in the Netherlands. In: M.L. Stenström \& P. Tynjälä (Eds.): Towards Integration of Work and Learning. New York, Berlin.

Rosendahl, J. \& Straka, G. A. (2007): Aneignung beruflicher Kompetenz - interessengeleitet oder leistungsmotiviert? Institut Technik und Bildung (ITB), Forschungsbericht 24. Universität Bremen.

Schnotz, W. \& Lowe, R. K. (2008). A Unified View of Learning from Animated and Static Graphics. In: R. K. Lowe \& W. Schnotz (Eds.): Learning with Animation. Research Implications for Design. Cambridge pp. 304-356.

Schwan, S. \& Riempp, R. (2004): The Cognitive Benefits of Interactive Videos: Learning to Tie Nautical Knots. In: Learning and Instruction, 14, pp. 293-305.

Seifried, J./Wuttke, E./Nickolaus, R./Sloane, P.F.E. (2010): Lehr-Lern-Forschung in der kaufmännischen Berufsbildung. ZBW Beiheft 23. Stuttgart. 
Tenberg, R. (2011): Vermittlung fachlicher und überfachlicher Kompetenzen in technischen Berufe. Stuttgart.

Volet, S. \& Järväla, S. (2001) (Eds.): Motivation in Learning Contexts. Amsterdam. Vygotsky, L. S. (1962): Thought and Language. Cambridge, MA.

Zinn, B. (2010): Ein Einblick in die wissensbezogenen Überzeugungen von Auszubildenden in gewerblich-technischen Berufsfeldern. In: Berufsbildung, H. 124, S. 45-47. 



\title{
Unterrichtliche Monotonie als Bedingungsfaktor für Schülerlangeweile im Fach Rechnungswesen
}

\author{
Kristina Kögler, Eveline Wuttke
}

\section{Problemstellung}

Langeweile - ob im Alltag oder im Unterricht - hat jeder schon erlebt. Besonders im Unterricht scheint sie allerdings ein Problem zu sein. Sucht man beispielsweise in Chat-Foren nach "Langeweile im Unterricht" findet man unzählige Hinweise wie diese erlebt wird und hunderte von Tipps, wie ihr zu begegnen sei. Und will man Treffer in Suchmaschinen als Gradmesser für die Aktualität von Themen sehen, ist Langeweile im Unterricht in der Tat ein zentrales Problem (weit über 200.000 Nennungen).

Erstaunlicherweise zeigt ein Blick auf die berufs- und wirtschaftspädagogische Forschung noch wenig Erhellendes zum Thema Langeweile. Aus Studien in anderen Domänen weiß man allerdings, dass häufig ein mittleres bis hohes Ausmaß an Langeweile im Unterricht zu finden ist. Schüler langweilen sich nach eigenen Angaben 30\% bis 60\% der Unterrichtszeit (vgl. Larson \& Richards 1991; Götz, Frenzel \& Pekrun 2007). Potentiell negative Folgen von Langeweile sind u.a. geringe Schulzufriedenheit, Abfall der Leistungsmotivation und des positiven emotionalen Befindens, Beeinträchtigung des schulischen Erfolgs und Absentismus bis hin zu Schulabbruch (Hill \& Perkins 1985; Götz et al. 2007). Bei der Entstehung von Langeweile spielen verschiedene Faktoren eine Rolle, insbesondere scheinen aber situationale Determinanten zentral zu sein. Dazu zählen Monotonie in der Unterrichtsgestaltung, das Ausmaß der Lehrerredezeit und auch Unterrichtsinhalte (z.B. Hill \& Perkins 1985; Götz, Frenzel \& Haag 2006; Wuttke 2008). Diese situationalen Determinanten sind auch in der beruflichen Bildung neuralgisch, vor allem zum Rechnungswesenunterricht. Aus neueren Studien ist bekannt, dass dort häufig Methodenmonismus und eine starke Schemaorientierung vorherrschen (Seifried 2009). Interessant ist deshalb die Frage, inwiefern Rechnungswesenunterricht eine besondere Anfälligkeit für methodische Monotonie und damit für Schülerlangeweile besitzt.

$\mathrm{Zu}$ den bereits genannten Erkenntnisdefiziten im Bereich der beruflichen Bildung kommt noch hinzu, dass prozessorientierte Forschungszugänge insgesamt weitgehend fehlen (zum Forschungsstand s. Lohrmann 2008a, b; Wuttke 2010). Die dem vorliegenden Beitrag zugrundeliegende Studie setzt sich deshalb zum Ziel, die methodische Gestaltung von Rechnungswesen- 
unterricht und das in diesem Unterricht vorzufindende Langeweileaufkommen prozessanalytisch zu untersuchen.

\section{Das Langeweilekonstrukt und die Bedeutung methodischer Abwechslung im Unterricht}

\subsection{Zum Stand der Langeweileforschung}

Versucht man Langeweile begrifflich zu fassen, wird schnell deutlich, dass es weder eine eindeutige Begriffsbestimmung noch eine tragfähige theoretische Basis gibt. Häufig wird Langeweile als negativ beschrieben, wenn auch durchaus positive Begleiterscheinungen (z.B. Reflexion und Entspannung, Harris 2000; Handelsinitiierung, Vodanovich 2003; Selbstreflexion, Seib \& Vodanovich 1998) und Folgen (Kreativität, die aus der „langweiligen“ Inkubationszeit heraus entsteht, Kast 2003; Schomäcker 2011) diskutiert werden. Eingeordnet wird sie als Emotion, der definitorische Minimalkonsens scheint zu sein, dass bei Langeweile subjektiv Zeitdilatation wahrgenommen und sie als verhältnismäßig negativ erlebt wird (Götz 2004; Pekrun 2006). Verglichen mit anderen Emotionen ist untypisch, dass sonst die Intensität sowohl positiver als auch negativer Emotionen mit zunehmender Wichtigkeit einer Situation zunimmt, bei Langeweile aber sinkt (Götz et al. 2007). Bei Langeweile handelt es sich zusammenfassend um ein affektives Erleben, das von negativer Valenz (,unangenehmes Gefühl“), Mangel an Interesse und Stimulation sowie meist niedrigem physiologischen Arousal geprägt ist (Mikulas \& Vodanovich 1993). Zudem möchte man die Langeweile erzeugende Situation durch behaviorale (nach Hause gehen) oder mentale Flucht (z.B. Tagträume) verlassen (Götz \& Frenzel 2006; Johnstone \& Scherer 2000; Wuttke 2008).

Bezüglich der Ursachen von Langeweile im Unterricht existieren international drei Modelle ${ }^{1}$. In diesen werden neben subjektiv erlebter Kontrolle von Situationen und Valenzen von Lernen und Leistung bzw. des jeweiligen Faches, die eher der Person des Lernenden zuzuordnen sind ${ }^{2}$, vor allem schulische Umweltfaktoren angeführt (Ausmaß an Kompetenzunterstützung,

1 Das sozialkognitive Modell der Entwicklung von Lern- und Leistungsemotionen (Pekrun 2000, 2006), ein situationsunspezifisches Modell von Hill und Perkins (1985) und ein nicht näher benanntes Modell von Robinson (1975).

2 Solche Persönlichkeitsmerkmale scheinen dafür verantwortlich zu sein, dass Menschen sich bei gleichen situativen Gegebenheit unterschiedlich schnell langweilen, d.h. unterschiedlich ,anfällig" sind für Langeweile (vgl. O’Hanlon 1981). Auch die Passung von Umwelt- und Personmerkmalen wurde häufig im Hinblick auf ein für Lern- und Leistungsprozesse optimales Level an Arousal bzw. Stimulation thematisiert (Hamilton 1981; Larson \& Richards 1991; O’Hanlon 1981). Derartige Passungen spielen auch für Flow-Erleben eine zentrale 
Leistungs- und Zielstrukturen, vgl. Pekrun 2000, 2006; monotone Situationen, Freiheitsgrade in einer Situation, Möglichkeiten eines Aufgabenwechsels, vgl. Hill \& Perkins 1985; monotoner Unterricht, Lehrer und Peers, vgl. Robinson 1975). Ähnliche Ursachen findet man in weiterer einschlägiger Literatur, ohne dass diese in Modelle eingebunden wären (Monotonie, repetitive Tätigkeiten, inadäquate Stimulation durch die Außenwelt, äußerer Zwang, vgl. Geiwitz 1966; Smith 1981; Vodanovich \& Kass 1990).

Befunde aus aktuellen Studien zeigen, dass die Unterrichtsgestaltung eine zentrale Quelle für Langeweileerleben sein kann (Daschmann, Goetz \& Stupnisky 2011). Werden Schüler gebeten, sich eine besonders langweilige Stunde zu vergegenwärtigen, dann beziehen sie sich überzufällig häufig auf eine Stunde, in der lehrerzentrierter Frontalunterricht durchgeführt wurde (Götz et al. 2007; Wuttke 2008). Langeweile kann in solchen Stunden sowohl durch Unterforderung als auch durch Überforderung entstehen (vgl. Sparfeldt, Buch, Kolender \& Rost 2011).

\subsection{Methodische Gestaltung des Unterrichts}

Betrachtet man die Unterrichtspraxis im Rechnungswesenunterricht, werden einige Schwachstellen und Grenzen deutlich, die sich insbesondere auch auf das Erleben von Langeweile durchschlagen könnten. An dieser Stelle soll die fachdidaktisch-curriculare Komponente ausgeklammert bleiben (s. dazu Seifried 2009), fachdidaktisch-methodisch ist die lehrerzentrierte und in kleine Lernschritte gegliederte Vorgehensweise im Rechnungswesen als problematisch einzustufen (Reinisch 1981; Reimers 1990; Tramm, Hinrichs \& Langenheim 1996; Seifried 2009). Grundsätzlich, d.h. nicht nur im Rechnungswesenunterricht, scheint in der Unterrichtsgestaltung eine „methodische Monostruktur" vorzuherrschen (Hage et al. 1985; Pätzold et al. 2003; Seifried 2009).

In der einschlägigen didaktisch-methodischen Literatur wird dagegen häufig lapidar auf die Notwendigkeit des Methodenwechsels verwiesen, wie das Zitat „die beste Methode ist der Methodenwechsel“ (Tütermann 1975: 176) veranschaulicht. Schon lange ist allerdings bekannt, dass der Wechsel alleine nicht zielführend ist, denn Dynamik in der Unterrichtsmethodik erzeugt ein Spannungsfeld aus Anregung und Unruhe. So führte die Suche nach dem „optimalen Variationswert“ (Dauenhauer 1970) nicht zu generalisierbaren Resultaten.

Rolle, welches Csikszentmihalyi (1975) als das „Gegenteil“ von Langeweile bezeichnet. Langeweile sollte entstehen, wenn die Passung nicht gegeben ist, v.a. wenn die Umwelt stark unterfordert (zur Langeweile aus Über- und Unterforderung s. auch Sparfeldt, Buch, Kolender \& Rost 2011). 
Inwieweit methodische Wechsel und Methodenvielfalt mit Langeweile zusammenhängen, soll im weiteren Verlauf dieses Beitrags geklärt werden. Methodenvielfalt bezieht sich dabei auf methodische Grundformen (z.B. Klassengespräch, Einzelarbeit, Partnerarbeit oder Gruppenarbeit), didaktische Arbeitsphasen (z.B. Erarbeitung, Wiederholung, Zusammenfassung), Handlungsmuster (z.B. Lehrervortrag, Unterrichtsgespräch, Schülervortrag) und Inszenierungstechniken (vormachen, zeigen, abstrahieren).

\section{Fragestellungen und Konzeption der Untersuchung}

Vor diesem theoretischen Hintergrund fokussiert der vorliegende Beitrag folgende Forschungsfragen zur Generierung erster Befunde für das beschriebene Problemfeld: (1) In welchem Ausmaß langweilen sich Lernende im Rechnungswesenunterricht? (2) Lassen sich didaktische Parameter in der Unterrichtsgestaltung identifizieren, die bei Abwechslungsarmut einen Zusammenhang zur Schülerlangeweile aufweisen?

Der empirische Zugang erfolgte im Rahmen einer prozessorientierten Videostudie in einer kaufmännischen Wirtschaftsschule in Franken. Zur Beobachtung der Unterrichtsprozesse im Fach Rechnungswesen wurden in vier Klassen ( $\mathrm{n}=96$ Lernende), davon zwei Notebookklassen, 32 Unterrichtsstunden videographiert, die Schüler-Schüler-Interaktion aufgezeichnet und das Unterrichtserleben von Lehrkräften und Lernenden in 7-minütiger Taktung mittels der Continuous-State-Sampling-Methodik (im Überblick Sembill, Seifried \& Dreyer 2008) erfasst. Die Untersuchung lehnte sich bezüglich der Erhebung der Erlebensdaten in Grundzügen an vorangegangene Prozessanalysen im Bereich des Selbstorganisierten Lernens und der Subjektiven Theorien von Lehrpersonen an (Sembill, Wuttke, Seifried, Egloffstein \& Rausch 2007; Seifried 2004; Seifried 2009): Es wurde für die verschiedenen Komponenten der emotionalen Befindlichkeit je ein kognitiv, emotional und motivational akzentuiertes Item eingesetzt. Darüber hinaus wurden spezifische Qualitäten des Zeiterlebens erhoben, das Item zur Schülerlangeweile lautete „Mir ist langweilig“. Die Lernenden waren alle 7 Minuten aufgefordert, die entsprechenden Items auf einer Skala von 0 bis $100 \mathrm{zu}$ beantworten, so dass sich für jeden Lernenden im Untersuchungszeitraum ca. 50 Messzeitpunkte (MZP) ergeben. Die resultierende Datenlage ermöglicht sowohl Analysen des individuellen Erlebens (einschließlich der Wechselwirkungen zwischen Erlebensdimensionen und MZP) als auch die Kopplung mit Beobachtungsdaten aus dem Unterricht zur Ermittlung systematischer Zusammenhangsmuster (s. z.B. Wild 2003).

Die beteiligten Klassen wiesen hinsichtlich ihrer Altersstruktur sowie dem fachinhaltsspezifischen Vorwissen in Rechnungswesen homogene Ein- 
gangsvoraussetzungen auf. Bezüglich der Unterrichtsgestaltung wurden den Lehrkräften keine didaktischen Vorgaben im Sinne der Interventionsforschung gemacht. Die Beschreibung methodischer Parameter der Unterrichtsgestaltung wurde im Rahmen einer systematischen Videoanalyse mit der Software Videograph (Rimmele 2002, 2011) durchgeführt und auf Basis eines zehnsekündigen Kodierintervalls im Time-sampling-Verfahren kodiert. Die Kategorisierung sichtstruktureller Aspekte des Unterrichtsgeschehens berücksichtigte neben Sozialformen (Plenumsarbeit, Gruppenarbeit, Partnerarbeit, Einzelarbeit) und Arbeitsphasen (Erarbeitung, Wiederholung, Sicherung) auch die Lehrerredezeit als methodisches Handlungsmuster; diese wurde allerdings mittels Event-Sampling festgestellt. Ungenutzte Zeiträume im Unterricht (z.B. für Unterbrechungen/Organisatorisches) wurden jeweils mit der Kategorie „Keine“ kodiert.

Die Operationalisierung von unterrichtsmethodischer Monotonie bzw. Abwechslungsarmut erfolgte vor dem Hintergrund der Erkenntnis, dass eine Festlegung auf starre Variationsmuster (z.B. im Sinne von zehnminütigen Methodenwechseln) im Hinblick auf Qualitätskriterien im Unterricht wenig Ziel führend ist. Es werden daher in einem ersten Zugriff zwei Facetten in den Blick genommen: (1) die maximale Länge eines methodisch konstanten Intervalls zwischen zwei Langeweile-Messzeitpunkten und (2) die relative Häufigkeit bestimmter didaktischer Kombinationen vor dem jeweiligen Messzeitpunkt.

\section{Befunde}

\subsection{Ausmaß der Schülerlangeweile im Rechnungswesenunterricht}

Zur Feststellung des Ausmaßes an Schülerlangeweile im beobachteten Unterricht wurden in einem ersten Analyseschritt die durch die Lernenden angegebenen Absolutwerte aller Messzeitpunkte aggregiert und die Mittelwerte und Streuungen der einzelnen Klassen miteinander verglichen (9A: $M=39.83$, $\mathrm{SD}=32.22 ;$ 9B: $\mathrm{M}=49.16, \quad \mathrm{SD}=34.14 ; 9 \mathrm{C}: \mathrm{M}=25.25, \quad \mathrm{SD}=27.28 ; 9 \mathrm{D}$ : $\mathrm{M}=41.56, \mathrm{SD}=30.69$ ). Die Mittelwertunterschiede zwischen den Klassen sind statistisch signifikant, es zeigt sich eine mittlere Effektstärke $(\mathrm{F}=122.13$, $\mathrm{p}=.000, \eta 2=.074)$, insbesondere die Klassen $\mathrm{B}$ und $\mathrm{C}$ fallen durch vergleichsweise deutlich höhere (Klasse B) bzw. geringere (Klasse C) Langeweilewerte auf. Die hohen Standardabweichungen deuten auf ausgeprägte intersubjektive Unterschiede und situative Einflüsse hin, so dass im Rahmen des weiteren Vorgehens aus Gründen der Vergleichbarkeit zunächst eine z-Standardisierung vorgenommen wird und darüber hinaus die Werte differenziert nach einzelnen Unterrichtsstunden betrachtet werden. 
Eine erste einfache Klassierung der z-Werte in über- und unterdurchschnittliche Langeweilemessungen (überdurchschnittlich: > MW, unterdurchschnittlich: $\leq \mathrm{MW}$ ) und die Berechnung entsprechender Anteile ergibt für die vier Klassen ebenfalls ein recht heterogenes Bild: So bewegt sich der prozentuale Anteil überdurchschnittlicher Langeweilemessungen in einem Bereich von knapp unter 30\% (Klasse C) bis nahezu 60\% (Klasse B) (vgl. Tab. 1). Aufgrund der äquidistanten Messzeitpunkte lässt sich dieses Ergebnis als prozentualer Anteil an der Unterrichtszeit auffassen, d.h. in Klasse B langweilen sich die Lernenden in nahezu $60 \%$ der beobachteten Unterrichtszeit.

Tabelle 1: Über-und unterdurchschnittliche Langeweilemessungen

\begin{tabular}{lccc} 
Klasse & $\begin{array}{c}\text { Unterdurchschnittliche } \\
\text { Langeweile }\end{array}$ & $\begin{array}{c}\text { Überdurchschnittliche } \\
\text { Langeweile }\end{array}$ & Gesamt \\
\hline A & $530(53.0 \%)$ & $470(47.0 \%)$ & $1000(100 \%)$ \\
B & $403(41.7 \%)$ & $563(58.3 \%)$ & $966(100 \%)$ \\
C & $922(71.6 \%)$ & $366(28.4 \%)$ & $1288(100 \%)$ \\
D & $692(52.2 \%)$ & $634(47.8 \%)$ & $1326(100 \%)$ \\
\hline
\end{tabular}

Nimmt man nachfolgend nur die überdurchschnittliche Langeweile in den Blick und betrachtet überdies einzelne Unterrichtseinheiten, so sind auch im Zeitverlauf deutliche Unterschiede festzustellen: Die Werte steigen gegen Ende des Erhebungszeitraumes in einigen Fällen deutlich an, insbesondere die sechste Stunde wird von den Lernenden als besonders langweilig empfunden, während die früheren Stunden außer in Klasse B als weniger langweilig erlebt werden (vgl. Tab. 2; die Prozentwerte beziehen sich auf die Anzahl der überdurchschnittlichen Langeweilemessungen in der betreffenden Einzel- oder Doppelstunde). Auffallend ist wiederum die Klasse B, in der sich die Lernenden in allen Unterrichtseinheiten in über 50\% der Unterrichtszeit überdurchschnittlich langweilen, während in Klasse C nur etwa ein Viertel bis ein Drittel des Unterrichts als besonders langweilig erlebt wird. In Anbetracht des auffallend hohen Ausmaßes an Schülerlangeweile in Klasse B wird für die nachfolgende Analyse der methodisch-didaktischen Gestaltung und Vielfalt im Unterricht diese Klasse herangezogen. 
Tabelle 2: Überdurchschnittliche Langeweile in Prozent der Unterrichtszeit

\begin{tabular}{lcccccccc} 
Stunde & $\mathbf{1}$ & $\mathbf{2}$ & $\mathbf{3}$ & $\mathbf{4}$ & $\mathbf{5}$ & $\mathbf{6}$ & $\mathbf{7}$ & $\mathbf{8}$ \\
\hline $\mathrm{A}(\mathrm{n}=1000)$ & 25.0 & 47.5 & 42.7 & 55.0 & 59.2 & 51.9 \\
\hline $\mathrm{B}(\mathrm{n}=966)$ & 63.3 & \multicolumn{2}{c}{52.4} & 51.6 & 62.7 & 67.5 & 60.8 \\
\hline $\mathrm{C}(\mathrm{n}=1288)$ & 25.6 & 26.8 & 29.8 & 29.2 & 30.4 & 31.5 & 27.4 & 25.9 \\
\hline $\mathrm{D}(\mathrm{n}=1326)$ & \multicolumn{2}{c}{38.8} & 53.3 & \multicolumn{2}{c}{44.6} & 54.4 \\
\hline
\end{tabular}

\subsection{Monotonie als Bedingungsfaktor für Schülerlangeweile}

Der Frage nach methodisch-didaktischen Parametern, die bei Abwechslungsarmut Schülerlangeweile bedingen, soll sich zunächst über die Beschreibung sichtstruktureller Merkmale der Unterrichtsgestaltung in Klasse B angenähert werden. Die Verteilung der kodierten Unterrichtszeit auf Sozialformen und Arbeitsphasen ist den Tabellen 3a und b zu entnehmen. Der größte Zeitanteil entfällt bei den Sozialformen mit knapp 57\% auf die Plenumsarbeit. Einzelund Partnerarbeitsphasen werden stundenweise wechselnd und in geringerem Ausmaß implementiert, die Einzelarbeit überwiegt dabei mit nahezu einem Fünftel der kodierten Unterrichtszeit. Gruppenarbeit wurde nicht implementiert. Auffallend ist zudem das Ausmaß der Leerläufe, insbesondere in Stunde 6 verstreichen große Anteile der Unterrichtszeit ungenutzt (Kategorie „Keine").

Tabelle 3a: Methodisches Unterrichtsprofil in Klasse B: Sozialformen

\begin{tabular}{lcccc} 
Stunde & \multicolumn{3}{c}{ Sozialformen (in \% der kodierten Unterrichtszeit) } \\
& keine & Plenumsarbeit & Einzelarbeit & $\begin{array}{c}\text { Partnerar- } \\
\text { beit }\end{array}$ \\
\hline 1 & 7.2 & 44.9 & 5.5 & 42.4 \\
$2 \_3$ & 2.7 & 58.2 & 32.6 & 6.5 \\
4 & 11.4 & 54.1 & 8.9 & 25.6 \\
5 & 11.1 & 61.1 & 27.9 & 0.0 \\
6 & 21.2 & 61.2 & 9.4 & 8.2 \\
78 & 16.2 & 57.7 & 17.1 & 9.0 \\
Gesamt & $\mathbf{1 0 . 8}$ & $\mathbf{5 6 . 6}$ & $\mathbf{1 9 . 0}$ & $\mathbf{1 3 . 6}$ \\
\hline
\end{tabular}

Die Verteilung der Arbeitsphasen weist ebenfalls ausgeprägte Leerläufe auf (insgesamt $15.3 \%$ ). Ausgiebig wird insbesondere gegen Ende der achtstündigen Inhaltseinheit wiederholt, während neue Inhalte, die etwa ein Drittel der Unterrichtszeit einnehmen, insbesondere in den frühen Stunden der Stoffeinheit erarbeitet und gesichert werden (vgl. Tab 3b). 
Tabelle 3b: Methodisches Unterrichtsprofil in Klasse B: Arbeitsphasen

\begin{tabular}{lcccc} 
& \multicolumn{4}{c}{ Arbeitsphasen (in \% der kodierten Unterrichtszeit) } \\
Stunde & keine & Wiederholung & Erarbeitung & Sicherung \\
\hline 1 & 15.7 & 0.0 & 47.0 & 37.3 \\
$2 \_3$ & 7.4 & 8.9 & 49.6 & 34.1 \\
4 & 17.9 & 35.8 & 35.8 & 10.6 \\
5 & 15.2 & 43.9 & 39.8 & 1.2 \\
6 & 23.9 & 60.5 & 15.6 & 0.0 \\
$7 \_8$ & 17.9 & 77.9 & 4.2 & 0.0 \\
Gesamt & $\mathbf{1 5 . 3}$ & $\mathbf{3 6 . 8}$ & $\mathbf{3 2 . 4}$ & $\mathbf{1 5 . 5}$ \\
\hline
\end{tabular}

Zur Häufigkeit der Methodenwechsel ist festzustellen, dass diese in den betrachteten Unterrichtseinheiten überaus häufig erfolgten - so wurde im Durchschnitt etwa alle 7 Minuten die Sozialform gewechselt, in der als besonders langweilig erlebten Stunde 6 fanden durchschnittlich gar alle vier Minuten Sozialformwechsel statt. Darüber hinaus erfolgte der Unterricht entlang typischer Muster - es ließen sich überzufällige didaktische Kombinationen aus Sozialform und Arbeitsphase identifizieren $\left(\chi^{2}=1717.72\right.$, $\mathrm{p}=.000)$ : Die Erarbeitung neuer Inhalte erfolgte überzufällig oft im Rahmen von Partner- und Einzelarbeitsphasen, während das Klassengespräch im Wesentlichen zur Wiederholung und Ergebnissicherung eingesetzt wurde (vgl. Abb. 1). Etwa ein Drittel der kodierten Einzelarbeitsphasen entfiel zudem auf die selbstständige Wiederholung von Inhalten.

Abbildung 1: Didaktische Muster des Unterrichts in Klasse B

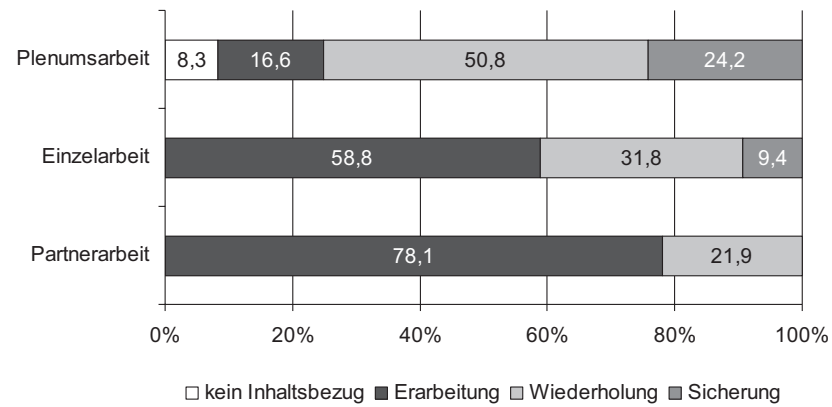

In einem nächsten Analyseschritt wurden die jeweils größten Zeitinvestments am Stück in den betrachteten methodischen Kategorien mit dem LangeweileErleben gekoppelt (1. Facette der Monotonie). Im Rahmen einer Korrelationsanalyse zeigte sich ein hochsignifikanter positiver Zusammenhang zwischen den ungenutzten Zeiträumen im Unterricht und der Schülerlangeweile 
(vgl. Tab. 4). Bei den Sozialformen Einzel- und Partnerarbeit und der Erarbeitung neuer Inhalte waren ebenfalls signifikante Zusammenhänge zu finden, allerdings stellen sich diese negativ dar - je länger diese am Stück implementiert wurden, desto weniger langweilten sich die Lernenden.

Tabelle 4: Methodische Abwechslungsarmut und Langeweileerleben

\begin{tabular}{|c|c|c|c|}
\hline \multirow{2}{*}{\multicolumn{2}{|c|}{$\begin{array}{l}\text { Größte Zeitinvestments am Stück für... } \\
\mathrm{n}=46\end{array}$}} & \multicolumn{2}{|c|}{ Prozesserleben Langeweile } \\
\hline & & $\begin{array}{c}\text { Korrelation } \\
\text { nach Pearson }\end{array}$ & $\begin{array}{l}\text { Signifikanz } \\
\text { 1-seitig }\end{array}$ \\
\hline \multirow{4}{*}{ Sozialformen } & Keine & $.501 * *$ & .000 \\
\hline & Plenumsarbeit & .056 & .356 \\
\hline & Einzelarbeit & $-.262 *$ & .039 \\
\hline & Partnerarbeit & $-.321 *$ & .015 \\
\hline \multirow{4}{*}{ Arbeitsphasen } & Keine & $.403 * *$ & .003 \\
\hline & Wiederholung & .181 & .114 \\
\hline & Erarbeitung & $-.446 * *$ & .001 \\
\hline & Sicherung & -.122 & 209 \\
\hline Handlungsmuster & Lehrervortrag/Instruktion & -.116 & .222 \\
\hline
\end{tabular}

Im Rahmen einer Drittvariablenkontrolle wurde durch Auspartialisieren überprüft, ob und inwiefern sich die gefundenen Zusammenhänge besonders für einige didaktische Kombinationen nachweisen lassen (2. Facette der Monotonie) oder übergreifende Geltung beanspruchen können. Es stellte sich heraus, dass lange Partnerarbeitsphasen nur dann als signifikant weniger langweilig wahrgenommen werden, wenn sie zur Erarbeitung neuer Inhalte verwendet werden $\left(\mathrm{r}=-.336^{*}, \mathrm{p}=.012\right)$, der positive Effekt verlor sich in Phasen der Wiederholung und Ergebnissicherung. Dieser Effekt zeigte sich in etwas abgeschwächter Form auch bei Einzelarbeitsphasen $\left(\mathrm{r}=-.299^{*}\right.$, $\mathrm{p}=.023$ ). Lange Wiederholungssequenzen wurden tendenziell als Langeweile erhöhend wahrgenommen, wenn sie im Rahmen der Plenumsarbeit durchgeführt wurden $(\mathrm{r}=.198, \mathrm{p}=.096)$.

\section{Diskussion und Ausblick}

Bezüglich des Ausmaßes der Schülerlangeweile im beobachteten Rechnungswesenunterricht konnten ältere Befunde repliziert werden, die von 30 bis $60 \%$ langweiliger Unterrichtszeit ausgehen. Auffallend sind dabei nicht nur große interindividuelle Streuungen und ausgeprägte Klassenunterschiede, sondern auch Veränderungen im zeitlichen Verlauf des Erhebungszeitraums. Es konnte exemplarisch für eine besonders intensiv gelangweilte Klasse ge- 
zeigt werden, dass sich Teile dieser Variabilität auf bestimmte didaktische Muster in der Unterrichtsgestaltung und das Ausmaß ungenutzter Zeiträume zurückführen lassen. So wirken lange Partner-/Einzelarbeits- und Stofferarbeitungsphasen Langeweile verringernd, während Wiederholungsphasen im Klassengespräch tendenziell Langeweile erhöhend sind.

Bei der Analyse der methodisch-didaktischen Unterrichtsgestaltung fielen in dieser Klasse zudem eine deutliche Dominanz lehrerzentrierter Phasen und ein hohes Ausmaß an Stoffwiederholungen auf. Überzufällig häufige didaktische Muster bestanden zum einen in der Kombination aus der Erarbeitung neuer Stoffinhalte in den Sozialformen Partner- und Einzelarbeit und zum anderen der Wiederholung bekannter Inhalte im Rahmen des Klassengesprächs. Zur Frage der methodischen Abwechslung konnte eine sehr hohe Dichte an Methodenwechseln festgestellt werden. Es drängt sich angesichts dessen der Eindruck auf, dass der vorliegende Unterricht nicht aufgrund seiner Abwechslungsarmut als langweilig erlebt wurde - so wurde es von den Lernenden unter gewissen Bedingungen sogar als Langeweile verringernd erlebt, sich auf eine Sache lange am Stück konzentrieren zu können - sondern vielmehr aufgrund der zahlreichen dicht getakteten Methodenwechsel in überzufälligen didaktischen Kombinationen und zahlreichen Leerläufen.

Dieses Ergebnis ist durch die ergänzende Berücksichtigung der Unterrichtsgestaltung in den anderen beteiligten Klassen im Rahmen der weiteren Datenauswertung zu überprüfen. Auch ein weiterführender Blick in korrespondierende Prozessmerkmale und inhaltsbezogene Aspekte des Unterrichts erscheint vielversprechend. Eine Weiterentwicklung der Operationalisierung von Monotonie durch die Hinzunahme von Reihenfolgebeziehungen und Methodensequenzen erscheint ebenfalls denkbar. Auf diesem Wege ließe sich die Frage vertiefen, in welcher Konstellation und Häufung Methodenwechsel im Unterricht von den Lernenden als positiv erlebt werden. Darüber hinaus ist in weiteren Analyseschritten die interindividuelle Varianz des Langeweileerlebens durch den Einbezug von Persönlichkeitsmerkmalen und Vorwissensbeständen der Lernenden in die Analyse situativer Langeweiledeterminanten zu berücksichtigen. 


\section{Literatur}

Csikszentmihalyi, M. (1975). Beyond Boredom and Anxiety. The experience of Play in Work and Games. San Francisco, Washington \& London: Jossey-Bass Publishers.

Daschmann, E. C., Goetz, T., \& Stupnisky, R. H. (2011). Testing the predictors of boredom at school. Development and validation of the Precursors to Boredom Scales. British Journal of Educational Psychology, 81, pp. 421-440.

Dauenhauer, E. (1970). Kategoriale Didaktik. Rinteln, München: Merkur.

Geiwitz, P. J. (1966). Structure of boredom. Journal of Personality and Social psychology, 3, pp. 592-600.

Götz, T. (2004). Emotionales Erleben und selbstreguliertes Lernen bei Schülern im Fach Mathematik. München: Utz.

Götz, T. \& Frenzel, A. (2006). Phänomenologie schulischer Langeweile. Zeitschrift für Entwicklungspsychologie, 38(4), S. 149-153.

Götz, T., Frenzel, A. C. \& Haag, L. (2006). Ursachen von Langeweile im Unterricht. Empirische Pädagogik, 20(2), S. 113-134.

Götz, T., Frenzel, A. C. \& Pekrun, R. (2007). Regulation von Langeweile im Unterricht Was Schülerinnen und Schüler bei der „Windstille der Seele“ (nicht) tun. Unterrichtswissenschaft, 35(4), S. 312-333.

Hage, K., Bischoff, H., Dichanz, H., Eubel, K.-D., Oehlschläger, H.-J. \& Schwittmann, D. (1985). Das Methoden-Repertoire von Lehrern Eine Untersuchung zum Schulalltag der Sekundarstufe I. Opladen: Leske + Budrich.

Hamilton, J. A. (1981). Attention, personality, and the self-regulation of mood: Absorbing interest and boredom. Progress in Experimental Personality Research, 10, pp. $281-351$.

Harris, M. B. (2000). Correlates and characteristics of boredom proneness and boredom. Journal of Applied Social Psychology, 30, pp. 576-598.

Hill, A. B. \& Perkins, R. E. (1985). Towards a model of boredom. British Journal of Psychology, 76(2), pp. 235-240.

Johnstone, T. \& Scherer, K. R. (2000). Vocal communication of emotion. In M. Lewis \& J. M. Haviland-Jones (Eds.), Handbook of emotions (pp. 220-235). New York: Guilford Press.

Kast, V. (2003). Vom Interesse und dem Sinn der Langeweile. München: dtv.

Larson, R. W. \& Richards, M. H. (1991). Boredom in the middle school years: Blaming schools versus blaming students. American Journal of Education, 99, pp. $418-443$.

Lohrmann, K. (2008a). Langeweile im Unterricht. Münster, New York, München, Berlin: Waxmann.

Lohrmann, K. (2008b). Langeweile im Unterricht. Ergänzende Darstellung des Forschungsstandes: Zusammenfassung von Einzelfallstudien. http://www.waxmann.com/kat/1896.html, Stand: 20.09.2011.

Mikulas, W. L. \& Vodanovich, S. J. (1993). The essence of boredom. Psychological record, 43, pp. 3-12.

O'Hanlon, J. F. (1981). Boredom: Practical consequences and a theory. Acta Psychologica, 49, pp. 53-82. 
Pätzold, G., Klusmeyer, J., Wingels, J. \& Lang, M. (2003). Lehr-Lern-Methoden in der beruflichen Bildung. Eine empirische Untersuchung in ausgewählten Berufsfeldern. Oldenburg: Universität Oldenburg.

Pekrun. R. (2000). A social-cognitive, control-value theory of achievement emotions. In J. Heckhausen (Ed.), Motivational psychology of human development (pp. 143-163). Oxford: Elsevier.

Pekrun, R. (2006). The control-value theory of achievement emotions: Assumptions, corollaries, and implications for educational research and practice. Educational Psychology Review. 18, pp. 315-341.

Reimers, J (1990). Grundzüge einer Didaktik des Rechnungswesens. In F. Achtenhagen (Hrsg.), Didaktik des Rechnungswesens. Programm und Kritik eines wirtschaftsinstrumentellen Ansatzes (S. 95-125). Wiesbaden: Gabler.

Reinisch, H. (1981). Entwicklung und Stand einer Fachdidaktik des einzelwirtschaftlichen Rechnungswesens. Wirtschaftswissenschaftliche Diskussionsbeiträge, Band 30, Oldenburg: Universität Oldenburg.

Rimmele, R. (2002/2011). Videograph - Multimedia-Player zur Kodierung von Videos. Kiel: IPN.

Robinson, W. P. (1975). Boredom at school. British Journal of Educational Psychology, 45, pp. 141-152.

Schomäcker, S. (2011). Schule braucht Langeweile? Über den Nutzen jugendlicher Langeweile für die Schule. Münster u.a.: Waxmann.

Seib, H. M. \& Vodanovich, S. J. (1998). Cognitive correlates of boredom proneness: The role of private self-consciousness and absorption. Journal of Psychology: Interdisciplinary and Applied, 132(6), pp. 642-652.

Seifried, J. (2004). Fachdidaktische Variationen in einer selbstorganisationsoffenen Lernumgebung - Eine empirische Untersuchung des Rechnungswesenunterrichts. Wiesbaden: DUV.

Seifried, J. (2009). Unterricht aus der Sicht von Handelslehrern. Frankfurt a. M.: Lang.

Sembill, D., Seifried, J. \& Dreyer, K. (2008). PDAs als Erhebungsinstrument in der beruflichen Lernforschung - Ein neues Wundermittel oder bewährter Standard? Empirische Pädagogik 22 (1), S. 64-77.

Sembill, D., Wuttke, E., Seifried, J., Egloffstein, M. \& Rausch, A. (2007). Selbstorganisiertes Lernen in der beruflichen Bildung - Abgrenzungen, Befunde und Konsequenzen. Berufs- und Wirtschaftspädagogik online - bwpat 13.

Smith, R. P. (1981). Boredom: A review. Human Factors, 23(3), S. 329-340.

Sparfeldt, J. R., Buch, S. R., Kolender, J. \& Rost, D. H. (2011). Überforderungs- und Unterforderungslangeweile in Mathematik: Differenzierung und Korrelate. In M. Dresel \& L. Lämmle (Hrsg.), Motivation, Selbstregulation und Leistungsexzellenz. (Talentförderung - Expertiseentwicklung - Leistungsexzellenz, Bd. 9, S. 53-70). Münster: LIT.

Tramm, T., Hinrichs, K. \& Langenheim, H. (1996). Lernschwierigkeiten im Buchführungsunterricht. In P. Preiß \& T. Tramm (Hrsg.), Rechnungswesenunterricht und ökonomisches Denken. Didaktische Innovationen für die kaufmännische Ausbildung (S. 158-221). Wiesbaden: Gabler.

Tütermann, H. (1975). Der Wechsel in der Methode ist die beste Unterrichtsmethode. Erziehungswissenschaft und Beruf, 23 (2), S. 172-177. 
Vodanovich, S. J. (2003). On the Possible Benefits of Boredom: A Neglected Area in Personality Research. Psychology and Education: An Interdisciplinary Journal, 40 , pp. $28-33$.

Vodanovich, S. J. \& Kass, S. J. (1990). A factor analytic study of the boredom proneness scale. Journal of personality assessment, 55, pp. 115-123.

Wild, K.-P. (2003). Videoanalysen als neue Impulsgeber für eine praxisnahe prozessorientierte empirische Unterrichtsforschung. Unterrichtswissenschaft 31(2), S. $98-102$.

Wuttke, E. (2008). Einige Selbstverständlichkeiten des Alltags und ihre theoretische und empirische Fundierung. Wirtschaft \& Erziehung, 6, S. 167-175.

Wuttke, E. (2010). Was wissen Lehrerinnen und Lehrer über Langeweile? Eine explorative Studie. Die berufsbildende Schule, 62, 11/12, S. 312-318. 



\title{
Die Lernwirksamkeit von einfachem und elaboriertem Feedback in Verbindung mit dem Erstellen von Concept Maps im Planspielunterricht
}

\author{
Jeannine Ryssel
}

\section{Problemstellung}

Komplexe Lehr-Lernarrangements wie z.B. Planspiele sind ein fester Bestandteil im Unterricht geworden. Dabei sollte der Planspieleinsatz, z.B. durch Concept Mapping als Lernstrategie, unterstützt werden. Auf diese Weise kann eine tiefgehende Verarbeitung der Inhalte erreicht werden. Concept Maps, als zweidimensionale Diagramme, bestehen aus KonzeptRelation-Konzept-Verbindungen (Propositionen), die darauf zielen Wissen und/oder Informationen darzustellen oder zu organisieren (Novak \& Cañas, 2008, S. 1). Hierbei stellt sich die Frage, inwieweit das Erstellen der Concept Maps allein eine Tiefenverarbeitung der erlernten Inhalte fördert oder ob es eines zusätzlichen Feedbacks bedarf. Dass Feedback eine entscheidende instruktionale Maßnahme ist, hat umfangreiche Forschung gezeigt (z.B. Hattie \& Timperley, 2007). Dabei wurden jedoch überwiegend computergestützte Lernumgebungen und gut strukturierte Probleme untersucht. Die Wirkung von Feedback beim Lernen komplexer Problemstellungen fand hingegen bisher kaum Beachtung.

Der Begriff Feedback stammt aus der Kybernetik, der Lehre von den Regelungswissenschaften, und ist zentraler Bestandteil des Regelkreismodells (Fengler, 2009, S. 12). Informationen werden, resultierend aus dem Vergleich des Ist- mit dem Soll-Zustand, rückgemeldet. Übertragen auf LehrLernprozesse bildet die Diskrepanz zwischen dem Ist-Zustand und dem zu erreichenden Lernziel die Basis für die Regulation des Lernprozesses (Krause, 2007, S. 46; Narciss, 2006, S. 17 f.). Ausgehend von diesem engen Begriffsverständnis haben die kognitive Wende sowie eine rasante Entwicklung computerbasierter Lehr-Lerntechnologien zu Begriffserweiterungen geführt (Narciss, 2006, S. 18). Dabei rückt zum einen die korrigierende Funktion des Feedbacks in den Vordergrund, zum anderen lassen sich neue FeedbackInhalte und -Formen mittels moderner Informationstechnologien gestalten und präsentieren (ebd.). Narciss (2006, S.18), Kluger und DeNisi (1996, S. 255) sowie Krause (2007, S. 50 f.) bezeichnen als Feedback alle Informationen, die Lernenden von einer externen Informationsquelle wie z.B. dem Leh- 
rer, dem Lernprogramm oder dem Versuchsleiter während oder nach der Aufgabenbearbeitung angeboten werden:

a) zur Fehlerkorrektur oder Bestätigung korrekter Antworten um vorgegebene Lernziele zu erreichen,

b) um Auskunft über den eigenen Lernfortschritt zu erhalten oder

c) um die eigene Leistung bzw. eigene Stärken und Schwächen mit anderen Lernenden zu vergleichen.

Dabei ist zu betonen, dass sich diese Informationen auch indirekt auf die Diskrepanz zwischen Soll und Ist beziehen können, z.B. auf das Beheben dieser Diskrepanz (Narciss 2006, S.18). Davon abzugrenzen ist internes Feedback, welches der Lernende aufgrund von Erfahrungen selbst generieren kann (Ilgen, Fisher \& Taylor, 1979, S. 351).

Feedback hat kognitive, metakognitive und motivationale Funktionen (siehe hierzu z.B. Narciss, 2006, S. 78 ff.) in Abhängigkeit von der Ebene im Lernprozess. Wird der Lernende bspw. über den Ort oder die Art eines Fehlers informiert oder werden ihm weitere Informationen bereitgestellt, die eine Korrektur von fehlerhaften Wissenselementen ermöglichen, so handelt es sich um kognitive Funktionen (ebd.). Sobald Informationen angeboten werden, die sich auf Prozesse der Selbstregulation beziehen und somit das Erreichen eines Lernzieles ermöglichen, hat Feedback eine metakognitive Funktion (ebd.). Feedback in einer motivationalen Funktion beinhaltet eine evaluative Komponente und beeinflusst somit den Anreizwert für künftige Aufgaben derselben Art, was wertsteigernd oder -mindernd wirken kann (ebd.).

Feedback lässt sich nach verschiedenen Kriterien klassifizieren (vgl. u.a. Butler \& Winne, 1995; Ilgen, Fisher \& Taylor, 1979), z.B. hinsichtlich der Komplexität der Feedbacknachricht nach einfachem und elaboriertem Feedback (z.B. Narciss, 2006, S. 19 ff.). Im Vordergrund steht dabei, welche und wie viele Informationen dem Lernenden zur Verfügung gestellt werden. Einfaches Feedback unterscheidet zwischen Knowledge of Performance (KP), d.h. der Anteil der richtig gelösten Aufgaben wird rückgemeldet, Knowledge of Result (KR), d.h. eine Information darüber, ob die Antwort richtig oder falsch ist und Knowledge of Correct Response (KCR), d.h. die richtige Lösung wird mitgeteilt (ebd.). Elaboriertes Feedback lässt sich ebenfalls weiter unterteilen. Hier sind die Klassifikationsvorschläge jedoch weniger eindeutig. Narciss (2006, S. 23) fokussiert auf inhaltliche Aspekte und unterteilt in Knowledge on task constraints (KTC), d.h. aufgabenbezogene Informationen, bspw. zur Art der Aufgabe oder zu Teilaufgaben, Knowledge about concepts $(\mathrm{KC})$, d.h. Informationen, die sich auf aufgabenrelevantes konzeptuelles Wissen beziehen, z.B. das Erklären von Fachbegriffen, Knowledge about mistakes (KM), d.h. fehlerbezogene Informationen, bspw. wo welcher Fehler 
gemacht wurde und wo Fehlerursachen liegen, Knowledge on how to proceed $(\mathrm{KH})$, d.h. Informationen, die sich auf strategisches Wissen beziehen, welches für die Aufgabenlösung benötigt wird, z.B. Hinweise zu Lösungsstrategien sowie Knowledge on meta-cognition (KMC), Informationen, die für die Regulation des Lernprozesses notwendig sind, bspw. Hinweise zu metakognitiven Strategien.

Die Lernwirksamkeit von Feedback wird von zahlreichen Faktoren beeinflusst. Individuelle Eigenschaften der Lerner bestimmen dabei die Feedbackverarbeitung. Andere Faktoren lassen sich der Feedbackgestaltung zuordnen. Verschiedene theoretische Ansätze haben auf die Wirkungsweise von Feedback und den Einfluss dieser Faktoren fokussiert und damit versucht, die empirischen Befunde der Feedbackforschung zu erklären (z.B. Bangert-Drowns et al., 1991; Butler \& Winne, 1995; Kluger \& DeNisi, 1996; Narciss, 2006). Zusammenfassend werden an dieser Stelle einige zentrale Erkenntnisse kurz vorgestellt. Insbesondere die individuellen Faktoren der Lernenden beeinflussen die Verarbeitung von extern bereitgestelltem Feedback. Neben dem Vorwissen bestimmen dabei auch die Überzeugungen zum einen die Bildung einer subjektiven Aufgabenpräsentation durch den Lernenden und zum anderen die Verarbeitung der Feedbackinformationen. Bei der Auswahl der Feedbackinhalte sollten immer die Lernziele, Lerninhalte und Lernaufgaben beachtet werden. Nur so können die Informationen bereitgestellt werden, die zur Bewältigung einer Aufgabe und zum Erreichen der Lernziele führen.

Die Metaanalysen von Bangert-Drowns et al. (1991) sowie Kluger und DeNisi (1996) zeigen, dass insbesondere die Feedbackart und der Instruktionskontext (computergestützte Lernumgebung vs. traditioneller Unterricht) einen starken Einfluss auf Feedback haben. Die Ergebnisse von Studien, welche die Wirkung unterschiedlich komplexer Feedbackarten auf die Lernwirksamkeit von Feedback untersucht haben und im Rahmen computergestützter Lern-/Testumgebungen durchgeführt wurden, zeigen unabhängig vom Fachgebiet und den Versuchsteilnehmern eine Vorteilhaftigkeit elaborierten gegenüber einfachen Feedbacks (z.B. Huth, 2004; Moreno, 2004). Im klassischen Unterrichts-/Lernkontext konnte eine solche Vorteilhaftigkeit bis jetzt nicht nachgewiesen werden (z.B. Rakoczy et al., 2008). Eine geringe Anzahl an Studien im realen Unterricht sowie methodische Aspekte sprechen aber dafür, dass die Ergebnisse noch nicht verallgemeinerbar sind und diesbezüglich weiterer Forschungsbedarf besteht.

Ferner bestätigen die Ergebnisse mehrerer dieser Studien den Einfluss des Lernzielniveaus. So konnten Moreno (2004) und Krause (2007) zeigen, dass die Aneignung anwendbaren und transferfähigen Wissens durch elaboriertes Feedback begünstig wird. Krause (2007) sowie Stark, Kopp und Fischer (2009) stellten jeweils einen moderierenden Effekt des fachspezifischen Vorwissens fest, wobei eine Angleichung des Wissensniveaus 
durch elaboriertes Feedback lediglich bei Krause (2007) nachgewiesen werden konnte. Stark, Kopp und Fischer (2009) konnten dies nicht bestätigen.

\section{Empirische Untersuchung}

In der hier vorgestellten Studie wurde untersucht, ob einfaches Feedback das Lernen betriebswirtschaftlicher Zusammenhänge im Rahmen eines Planspiels besser als elaboriertes Feedback unterstützt. Die Selbstkonstruktion von Concept Maps diente dabei als Aufgabenformat sowie als Lernstrategie. Folgende Hypothesen wurden formuliert:

1. Elaboriertes Feedback ist lernwirksamer als einfaches Feedback. 2. Die Lernwirksamkeit unterschiedlich komplexer Feedbackarten variiert in Abhängigkeit vom Lernziel. 2a: Bei einfachen Lernzielen ist einfaches Feedback genauso lernwirksam wie elaboriertes Feedback. 2b. Bei komplexen Lernzielen ist elaboriertes Feedback lernwirksamer als einfaches Feedback.

Eingesetzt wurde das Planspiel „Easy Business ${ }^{\text {TM },, ~ w e l c h e s ~ a l s ~ B r e t t-~}$ spiel konzipiert ist und grundlegende betriebswirtschaftliche Zusammenhänge vermittelt. Ausgehend von der Simulation des Produktionsprozesses eines Fertigungsbetriebes erfahren die Schüler, welche Auswirkungen ihre Handlungen und Maßnahmen in der jährlichen Rechnungslegung haben. Insgesamt nahmen 41 Schüler zweier achter Klassen der Mittelschule Neustadt (Sachsen) im Jahr 2010 an der Studie teil. Das Durchschnittsalter der Probanden betrug 13,6 Jahre. Die Schüler wurden zufällig zwei Gruppen zugeordnet.

Der Ablauf lässt sich dabei als Dreischritt auffassen, bestehend aus einer Planspielphase, einer darauffolgenden Concept Mapping-Aktivität und einer zweifach gestuften Feedbackmaßnahme. In den einzelnen Planspielphasen wurden die betriebswirtschaftlichen Inhalte im Rahmen von Spiel- und Systematisierungsphasen erarbeitet. Eingebettet in das Planspiel erstellten die Schüler Concept Maps zu den Spielinhalten. Hierbei wird der Forschung zur Lernwirksamkeit von Concept Maps Rechnung getragen, welche postuliert, dass Concept Mapping in den Lernprozess eingebettet und nicht nur als „Add-On“ am Beginn oder Ende dieses Prozesses eingesetzt werden sollte (Cañas, 2003, S. 8). Das erste Concept Map sollte dabei die Abläufe in einem Produktionsunternehmen abbilden. Im zweiten Concept Map stellten die Schüler die Zusammenhänge zwischen linearer Abschreibung, Gewinn- und Verlustrechnung und Bilanz her. Beim dritten Mapping sollten die Zusammenhänge zwischen dem ersten und dem zweiten Concept Map dargestellt werden.

Die Mapping Aktivitäten stellten dabei zum einen eine Lernstrategie dar, um den Planspieleinsatz zu unterstützen. Zum anderen dienten sie als Aufga- 
benformat und somit der Wissensdiagnose, d.h. sie erfassten, was die Schüler jeweils gelernt hatten. Dieser Aspekt wurde im Rahmen der Untersuchung fokussiert, um Feedback anbieten zu können. Die Erstellung der Concept Maps erfolgte mittels Papier und Bleistift. Den Probanden wurden Konzepte und Relationen in Form einer Liste zur Verfügung gestellt, mit deren Hilfe sie die Concept Maps konstruieren sollten. ${ }^{1}$

Die erste Gruppe erhielt zusätzlich einfaches Feedback in Form eines Vergleichs mit dem Referenzmap (KR und KCR). Die Schüler sollten sich intensiv mit dem Referenzmap auseinandersetzen und konnten in einer Kopie ihrer zuvor angefertigten Concept Maps Änderungen vornehmen. In der zweiten Gruppe wurden darüber hinaus Fehler exemplarisch anhand eines Schülernetzes ausgewertet sowie die Referenznetze in einem Lehrer-SchülerGespräch besprochen (elaboriertes Feedback in Form von KC und KM). Es handelt sich folglich um externes Feedback mit kognitiver Funktion.

Vor und nach der Intervention wurde ein Wissenstest als Paralleltest mit einfachen und komplexen Aufgaben durchgeführt. Die Fragen des Wissenstests wurden anhand der Taxonomie von Anderson und Krathwohl (2001) entwickelt. Sie zielten darauf zu prüfen, ob die Schüler Faktenwissen und konzeptuelles Wissen erinnern bzw. verstehen und ob sie in der Lage sind, prozedurales Wissen anzuwenden. Das Erinnern von Faktenwissen und konzeptuellem Wissen wird dabei als einfach eingestuft, konzeptuelles Wissen verstehen sowie prozedurales Wissen anwenden werden als komplex betrachtet. Diese Zuordnung ist ableitbar aus dem Vorwissensstand der Schüler. Im Vorfeld wurde der Wissenstest einer vorangegangen Untersuchung (Ryssel \& Fürstenau, 2011) angepasst sowie die Paralleltestreliabilität überprüft. Diese ist laut Lienert und Raatz (1998, S. 14) mit einem Wert von $r=0,79$ für das Forschungsinstrument ausreichend, um Gruppen hinsichtlich des zu untersuchenden Merkmals zu vergleichen.

Des Weiteren wurden auch die Daten des Lernprozesses, d.h. die erstellten sowie überarbeiteten Concept Maps, erfasst und ausgewertet. Abbildung 1 veranschaulicht den Untersuchungsaufbau.

1 In Vorbereitung auf die Untersuchung wurde die Methode des Concept Mapping anhand zweier Beispiele geübt. Als Hilfestellung erhielten die Probanden eine schriftliche Anleitung, welche die Vorgehensweise beim Erstellen eines Concept Maps anhand eines Beispiels demonstrierte. Hierbei wurde sich an Erfahrungen aus einer vorangegangenen Untersuchung orientiert (Ryssel \& Fürstenau, 2011). 


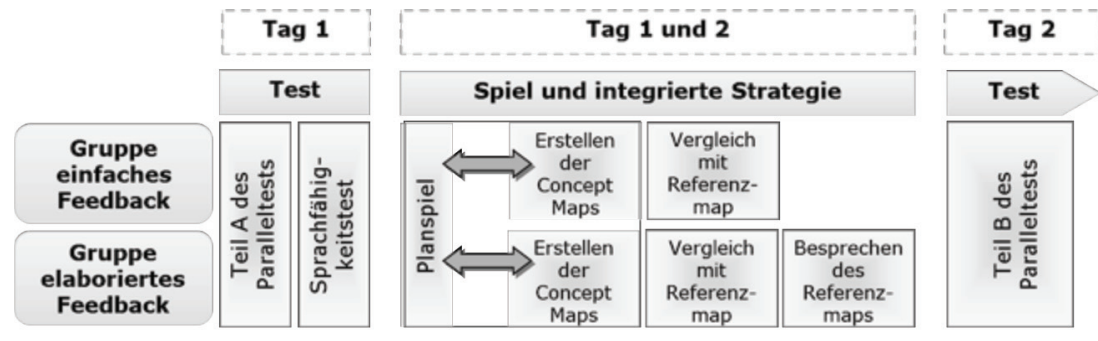

Die Daten der Wissenstests wurden mittels einer kategorialen Inhaltsanalyse ausgewertet. Die Intercoder-Reliabilität von 96,9 Prozent (Spearman-Rho) lässt auf die Qualität des verwendeten Instrumentariums und der damit verbundenen Messvorschriften sowie auf die Sorgfalt bei deren Anwendung schließen. Auf Basis der kategorialen Inhaltsanalyse konnte für jeden Probanden ein Testwert errechnet werden. Um festzustellen, ob Unterschiede im Wissenszuwachs zwischen Vor- und Nachtest durch die verschiedenen Treatmentbedingungen (H1) und/oder das Vorwissen (H3) erklärt werden können, wurde eine dreifaktorielle, univariate Varianzanalyse mit Messwiederholung (ANOVA) durchgeführt. Hierfür wurde die Variable „Vorwissen“ am Median dichotomisiert. ${ }^{2}$ Dementsprechend galt das Vorwissen als hoch, wenn es über dem Median und als niedrig, wenn es unter dem Median für beide Gruppen lag. Des Weiteren wurde je eine zweifaktorielle ANOVA mit Messwiederholung für einfache und komplexe Aufgaben vorgenommen. ${ }^{3}$ Darüber hinaus erfolgte die Berechnung von Effektstärken. Im Falle eines Pretest-Posttest-Designs können die Effektstärken mit folgender Formel berechnet werden: $d_{\text {int }}(=$ Interaktion $)=d_{\text {posttest }}-d_{\text {pretest }}$ (Klauer, 1993). Die gemittelte Standardabweichung der entsprechenden Gruppen wurde dabei als Standardabweichung verwendet.

\section{Ergebnisse der Untersuchung}

Auf Basis der dreifaktoriellen ANOVA mit Messwiederholung lässt sich zunächst ein signifikanter Haupteffekt für den Faktor "Zeit" nachweisen $(\mathrm{F}=75,107 ; \mathrm{p}=0,000)$. Demnach konnten alle Versuchsgruppen einen signifikanten Wissenszuwachs vom Vor- zum Nachtest erzielen. Insgesamt kann

2 Hierbei wird der üblichen Verfahrensweise zur Teilung der Probanden in Personen mit hohem und niedrigem Vorwissen gefolgt (Brünken \& Leutner, 2005, S. 32).

3 Für alle statistischen Analysen wurde ein Signifikanzniveau von 5\% gewählt. 
kein signifikanter Interaktionseffekt für die Faktoren „Zeit“ und „Gruppe“ nachgewiesen werden $(\mathrm{F}=1,055 ; \mathrm{p}=0,312)$. Dementsprechend unterscheiden sich die Feedbackbedingungen im Wissenszuwachs nicht signifikant voneinander. Der Vergleich der elaborierten Feedback- mit der einfachen Feedback-Gruppe führt mit einem Wert von $\mathrm{d}=0,0638 \mathrm{zu}$ einem sehr geringen Effekt. Es lassen sich Tendenzen ableiten, die einen geringen Vorteil des elaborierten Feedbacks zeigen. Die elaborierte Feedback-Gruppe (6,68 Punkte) erzielte im Vergleich zur einfachen Feedback-Gruppe (5,22 Punkte) einen höheren Wissenszuwachs. Die Ergebnisse sind in nachfolgender Abbildung 2 dargestellt.

Abbildung 2: Ergebnisse der dreifaktoriellen ANOVA mit Messwiederholung für Zeit und Gruppe (UV) und Testwert (AV)

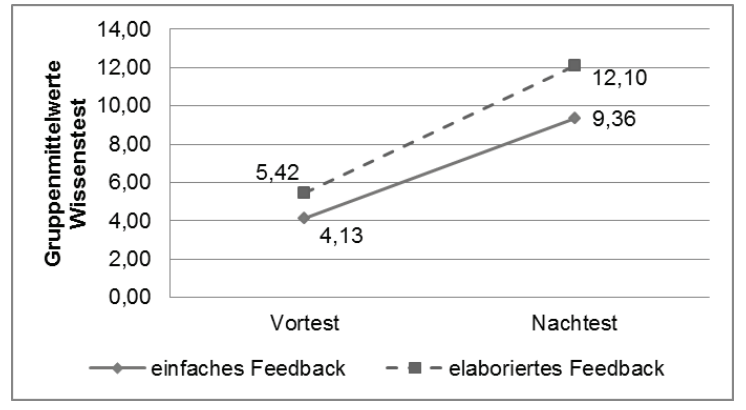

Die zweifaktorielle Varianzanalyse mit Messwiederholung bezüglich der Testwerte für einfache und komplexe Aufgaben als abhängige Variablen zeigte ein ähnliches Bild wie über alle Aufgaben. Es konnte jeweils ein signifikanter Haupteffekt für den Faktor „Zeit“ nachgewiesen werden ( F=144,363 bzw. $F=95,520, p=0,000$ ), d.h. beide Feedbackbedingungen erzielten sowohl bei den einfachen als auch bei den komplexen Aufgaben einen signifikanten Wissenszuwachs. Es konnte jeweils kein signifikanter Interaktionseffekt für die Faktoren „Zeit“ und „Gruppe“ gezeigt werden. Bei den komplexen Aufgaben wurde das Signifikanzniveau jedoch nur knapp verfehlt $(\mathrm{F}=0,019$, $\mathrm{p}=0,892 ; \mathrm{F}=3,684, \mathrm{p}=0,063)$. 
Abbildung 3: Ergebnisse der zweifaktoriellen ANOVA mit Messwiederholung für Zeit und Gruppe (UV) und Testwert bzgl. einfacher Aufgaben (AV)

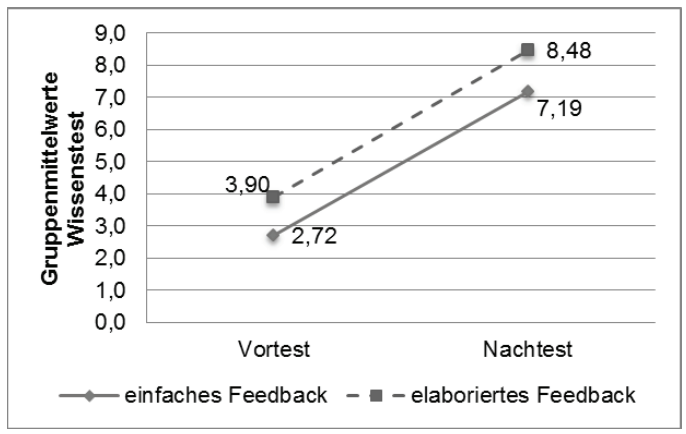

Abbildung 3 verdeutlicht, dass der Wissenszuwachs bezüglich einfacher Aufgaben bei beiden Feedback-Gruppen in etwa gleich ist $(4,47$ bzw. 4,58 Punkte). Die Berechnung der Effektstärke ergibt einen Wert von -0,1507, was einen kleinen negativen Effekt bedeutet.

Abbildung 4 zeigt, dass der Wissenszuwachs bei den komplexen Aufgaben für die Gruppe elaboriertes Feedback (4,43 Punkte) größer ist als für die Gruppe einfaches Feedback (2,97 Punkte). Die Effektstärke für den Wissenszuwachs kann mit einem Wert von $\mathrm{d}=0,5930$ als mittel bewertet werden. Es lassen sich folglich Tendenzen ableiten, die eine Wirksamkeit des elaborierten Feedbacks gegenüber einfachem Feedback bei komplexen Aufgaben zeigt. Unter Berücksichtigung der Taxonomiestufen ist erkennbar, dass die Gruppe elaboriertes Feedback bei „konzeptuelles Wissen verstehen“ einen signifikant höheren Wissenszuwachs erzielte als die Gruppe einfaches Feedback $(\mathrm{F}=5,848 ; \mathrm{p}=0,021)$.

Abbildung 4: Ergebnisse der zweifaktoriellen ANOVA mit Messwiederholung für Zeit und Gruppe (UV) und Testwert für komplexe Aufgaben (AV)

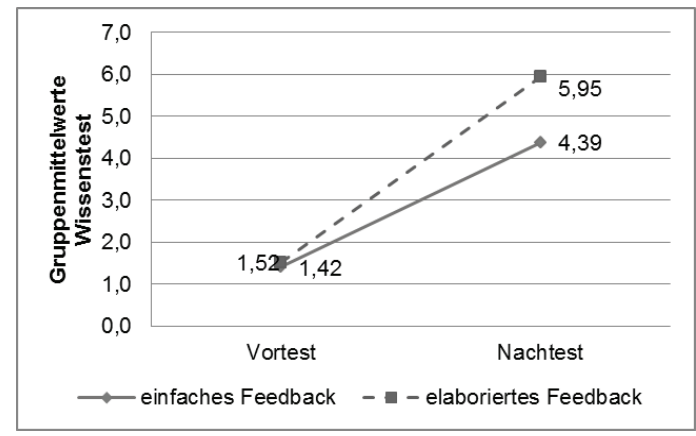


Bezüglich des Einflusses des Vorwissens im Zusammenhang mit der Feedbackbedingung auf den Wissenszuwachs zeigt die dreifaktorielle ANOVA mit Messwiederholung keine signifikanten Interaktionseffekte für die Faktoren „Zeit" und „Vorwissen“ ( $\mathrm{F}=0,008, \mathrm{p}=0,931)$ sowie „Zeit“, „Gruppe“ und „Vorwissen“ ( $\mathrm{F}=0,009, \mathrm{p}=0,924)$. Vorwissensstärkere Schüler unterscheiden sich nicht signifikant von den vorwissensschwächeren Schülern, auch unabhängig davon, welches Feedback sie erhielten. Aus Abbildung 5 ist erkennbar, dass der Wissenszuwachs zwischen den vier Gruppen nur geringfügig variiert. Tendenziell lässt sich ableiten, dass Probanden der elaborierten Feedback-Gruppe sowohl mit geringem als auch mit hohem Vorwissen gegenüber der einfachen Feedback-Gruppe einen höheren Wissenszuwachs erreichten (6,50 Punkte bzw. 6,75 Punkte im Vergleich zu 5,23 bzw. 5,21 Punkten).

Abbildung 5: Ergebnisse dreifaktoriellen ANOVA mit Messwiederholung für Zeit, Vorwissen und Gruppe (UV) und Testwert (AV)

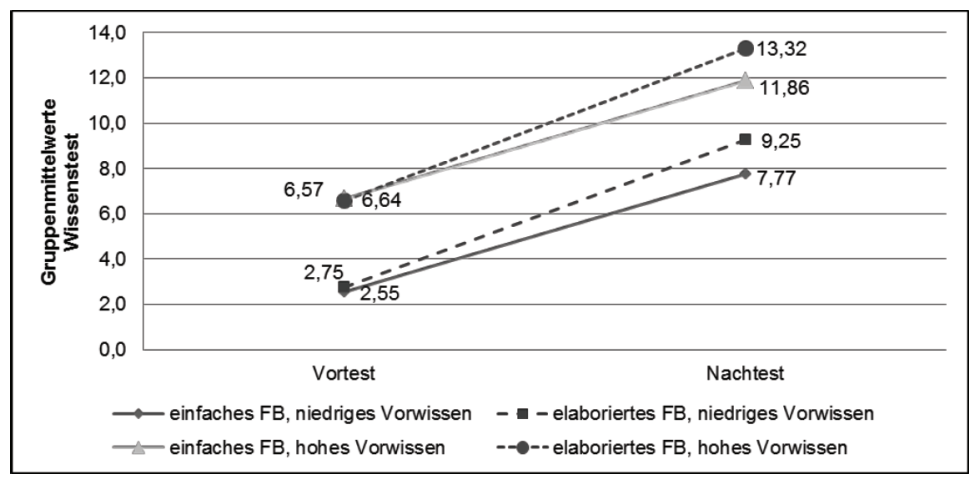

Die wichtigsten Ergebnisse der empirischen Untersuchung sind in nachfolgender Tabelle 1 zusammenfassend dargestellt. 
Tabelle 1: Ergebnisse der zweifaktoriellen Varianzanalysen

\begin{tabular}{|c|c|c|c|c|c|}
\hline Quelle & $\begin{array}{l}\text { Quadrat- } \\
\text { summe }\end{array}$ & df & $\begin{array}{l}\text { Mittel der } \\
\text { Quadrate }\end{array}$ & F-Wert & $\mathbf{P}$ \\
\hline \multicolumn{6}{|c|}{ Abh. Variable Testwert (über alle Aufgaben) } \\
\hline Zeit & 594,761 & 1 & 594,761 & 75,107 & $0,000^{*}$ \\
\hline Gruppe & 10,017 & 1 & 10,017 & 1,080 & 0,306 \\
\hline Vorwissen & 273,804 & 1 & 273,804 & 29,510 & $0,000^{*}$ \\
\hline Zeit x Gruppe & 8,353 & 1 & 8,353 & 1,055 & 0,312 \\
\hline Zeit $\mathrm{x}$ Vorwissen & 0,060 & 1 & 0,060 & 0,008 & 0,931 \\
\hline Zeit $x$ Gruppe $x$ Vorwissen & 0,073 & 1 & 0,073 & 0,009 & 0,924 \\
\hline \multicolumn{6}{|c|}{ Abh. Variable Testwert (über einfache Aufgaben) } \\
\hline Zeit & 387,721 & 1 & 387,721 & 144,363 & $0,000^{*}$ \\
\hline Gruppe & 28,627 & 1 & 28,627 & 4,180 & $0,048^{*}$ \\
\hline Zeit x Gruppe & 0,050 & 1 & 0,050 & 0,019 & 0,892 \\
\hline \multicolumn{6}{|c|}{ Abh. Variable Testwert (über komplexe Aufgaben) } \\
\hline Zeit & 259,195 & 1 & 259,195 & 95,520 & $0,000^{*}$ \\
\hline Gruppe & 13,202 & 1 & 13,202 & 3,108 & 0,086 \\
\hline Zeit x Gruppe & 9,997 & 1 & 9,997 & 3,684 & 0,063 \\
\hline
\end{tabular}

* signifikant auf dem Niveau $\alpha=0,05$

\section{Diskussion und Ausblick}

Es lassen sich insgesamt keine signifikanten Ergebnisse ableiten, welche eindeutig für eine allgemeine Vorteilhaftigkeit des elaborierten Feedbacks im Vergleich zum einfachen Feedback sprechen. Hypothese 1 muss demnach abgelehnt werden. Dies bestätigt Ergebnisse zu Studien im realen Unterrichtskontext (z.B. Racoczy et al., 2008). Tendenziell ist jedoch ein geringer Vorteil des elaborierten Feedbacks erkennbar.

Die Annahme, dass das Lernzielniveau die Wirkung der Feedbackmaßnahme moderiert, kann nur teilweise bestätigt werden. Bei einfachen Aufgaben zeigte sich kein signifikanter Unterschied zwischen den Feedbackgruppen hinsichtlich des Wissenszuwachses. Hypothese 2a kann demnach gestützt werden. Für weniger anspruchsvolle Lernziele scheint demnach einfaches Feedback in Form der Präsentation der richtigen Lösung auszureichen. Bezüglich komplexer Aufgaben unterscheiden sich die Feedbackbedingungen im Wissenszuwachs nicht signifikant voneinander. Hypothese 2a muss folglich abgelehnt werden. Das Signifikanzniveau wird 
jedoch mit einem Wert von $\mathrm{p}=0,062$ nur knapp verfehlt. Darüber hinaus zeigt die Effektstärke einen mittleren Effekt. Tendenziell kann also davon ausgegangen werden, dass komplexere Lernziele auch eines umfangreichen Feedbacks bedürfen. Eine differenzierte Betrachtung der Taxonomiestufen zeigt für das Verstehen von konzeptuellem Wissen einen signifikant höheren Wissenszuwachs der elaborierten Feedback-Gruppe. Der Einfluss des Lernzielniveaus ist folglich erkennbar. Diese Ergebnisse gehen konform mit theoretischen Erkenntnissen sowie der empirischen Befundlage.

Hinsichtlich des Vorwissens zeigt sich kein Einfluss auf den Wissenszuwachs auch unabhängig von der Feedbackbedingung. Eine Angleichung des Wissens durch die Feedbackmaßnahme kann daher nicht belegt werden. Hypothese 3 muss demnach abgelehnt werden. Hierbei besteht weiterer Forschungsbedarf. Möglicherweise war die elaborierte Feedbackmaßnahme für Schüler mit geringem Vorwissen zu unspezifisch, um zu einer größeren Leistungsverbesserung zu führen. In der hier vorgestellten Untersuchung wurden zwar Fehler exemplarisch besprochen. Dies deckte aber nur einen Teil der Fehlkonzepte auf. Ansonsten wurden Fehler nur durch Schülerantworten im Lehrer-Schüler-Gespräch thematisiert. Vor diesem Hintergrund soll eine Analyse durchgeführt werden, welche mögliche Fehlkonzepte, im Sinne von falschen und fehlenden inhaltlichen Zusammenhängen, aufdecken soll. Als Grundlage hierfür dienen die erstellten Concept Maps. Hierbei soll untersucht werden, welche Propositionen häufig genannt wurden, aber nicht im Referenznetz vorhanden sind. Diese müssen beim Feedback thematisiert werden. Des Weiteren gilt es herauszufinden, welche Propositionen kaum genannt wurden, jedoch Teil des Referenznetzes sind. Diesen Propositionen muss ebenfalls beim Feedback mehr Beachtung geschenkt werden. Auf diese Weise werden wichtige inhaltliche Zusammenhänge identifiziert, die bei Folgeuntersuchungen im Rahmen der Gestaltung der elaborierten Feedbackmaßnahme beachtet werden müssten. Das elaborierte Feedback könnte so für Folgeuntersuchungen verbessert werden.

Darüber hinaus soll analysiert werden, wie Schüler mit der richtigen Lösung (Referenznetz) umgehen, d.h. ob Propositionen einfach nur hinzugefügt oder auch falsche gelöscht bzw. korrigiert werden. Erste Analysen hierzu zeigen, dass besonders häufig Propositionen der Referenznetze unreflektiert übernommen wurden. Dies äußert sich darin, dass Verknüpfungen oft falsch bzw. unvollständig hinzugefügt wurden, was auf eine unzureichende Auseinandersetzung mit den Maps schließen lässt. Hierbei gilt es, die Instruktionsanweisungen der Versuchsleiter zu optimieren.

Insgesamt bleibt noch offen, inwieweit die Ergebnisse allein auf die Variation der Feedbackmaßnahme zurückzuführen sind. Hierbei ist anzumerken, dass die hier betrachtete Studie nur einen Teil einer größeren Untersuchung darstellt. Es wurde eine weitere Gruppe einbezogen, welche kein Feedback erhielt, sondern lediglich die Concept Maps erstellte. Die Ergebnisse der ge- 
nerellen Wirkung des Feedbacks sind folglich noch offen. Des Weiteren bleibt noch als Kontrollbedingung zu klären, welche Lernwirksamkeit die Lernstrategie Concept Mapping erzielte. Hierfür wurde mit einer weiteren Gruppe lediglich das Planspiel durchgeführt.

Darüber hinaus werden neben dem Vorwissen noch weitere Einflussfaktoren, wie die Sprachfähigkeit, die empfundene Nützlichkeit des Feedbacks, der Umgang mit der Methode des Concept Mapping sowie der Umgang mit Fehlern analysiert. Ebenso gilt es, die Qualität der Concept Maps zu betrachten. Insbesondere ist dabei interessant, inwieweit sich die Qualität des zweiten und dritten Concept Maps zwischen den Feedbackbedingungen unterscheidet. Eine höhere Qualität des zweiten bzw. dritten Concept Maps der Gruppe elaboriertes Feedback könnte auch auf eine höhere Lernwirksamkeit des elaborierten Feedbacks zurückzuführen sein.

Insgesamt besteht noch weitere Forschungsbedarf zur Wirksamkeit des Feedbacks insbesondere im realen Lehr-/Lernkontext. Dabei ist die Frage einer generellen Wirksamkeit nicht unbedingt zielführend, da diese durch vielschichtige Faktoren wie z.B. dem Lernzielniveau beeinflusst wird. Vor dem Hintergrund der immer komplexer werdenden Anforderungen des beruflichen Alltags an Lernende gilt es dabei geeignete Feedbackformate für vielschichtige und vernetzte Wissensinhalte sowie anwendbares Wissen bereitzustellen.

\section{Literatur}

Achtenhagen, F. (1992). Zur Evaluation komplexer Lehr-Lernarrangements als neue Formen des Lehrens und Lernens in beruflichen Schulen. In P. Gonon (Hrsg.), Evaluation in der Berufsbildung (S. 57-83). Aarau: Sauerländer.

Anderson, L. W. \& Krathwohl, D. R. (2001). A Taxonomy for Learning, Teaching, and Assessing: A Revision of Bloom's Taxonomy of Educational Objectives. New York: Addison Wesley Longman.

Bangert-Drowns, R. L., Kulik, C. C., Kulik, J. A. \& Morgan, M. T. (1991). The Instructional Effect of Feedback in Test-Like Events [Electronic Version]. Review of Educational Research, 61 (2), pp. 213-238.

Brünken, R. \& Leutner, D. (2005). Individuelle Unterschiede beim Lernen mit neuen Medien - neue Wege in der ATI-Forschung. In S. R. Schilling, J. R. Sparfeldt \& C. Pruisken (Hrsg.), Aktuelle Aspekte pädagogisch-psychologischer Forschung Detlef H. Rost zum 60. Geburtstag (S. 25-40). Münster: Waxmann.

Butler, D. L. \& Winne, P. H. (1995). Feedback and Self-Regulated Learning: A Theoretical Synthesis [Electronic Version]. Review of Educational Research, 65, pp. 245-281. 
Cañas, A. J. (2003). A Summary of Literature Pertaining to the Use of Concept Mapping Techniques and Technologies for Education and Performance Support. [Online]. Verfügbar unter: http:/www.ihmc.us/users/acanas/Publications/ConceptMapLitReview/IHMC\%20Literature\%20Review\%20on\%20Concept $\% 20 \mathrm{Ma}$ pping.pdf [2011-09-14].

Fengler, J. (2009). Feedback geben: Strategien und Übungen (4. Aufl.). Weinheim und Basel. Beltz.

Hattie, J. \& Timperley, H. (2007). The Power of Feedback [Electronic Version]. Review of Educational Research, 77, pp. 81-112

Huth, K. (2004). Entwicklung und Evaluation von fehlerspezifischem informativem tutoriellen Feedback (ITF) für die schriftliche Subtraktion. Dissertation, Technische Universität Dresden [Online]. Verfügbar unter http:/www.qucosa.de/ fileadmin/data/qucosa/documents/1243/1105354057406-4715.pdf [2010-09-12].

Ilgen, D. R., Fisher, C. D. \& Taylor, M. S. (1979). Consequences of Individual Feedback on Behavior in Organizations [Electronic Version]. Journal of Applied Psychology, 64, pp. 349-371.

Klauer, K. (1993). Denktraining für Jugendliche. Ein Programm zur intellektuellen Förderung. Handanweisung.

Kluger, A. N. \& DeNisi, A. (1996). The Effects of Feedback Interventions on Performance: A Historical Review, a Meta Analysis and a Preliminary Feedback Intervention Theory [Electronic Version]. Psychological Bulletin, 119, pp. 254284.

Lienert, G. A. \& Raatz, U. (1998). Testaufbau und Testanalyse (6. Aufl.). Weinheim: Beltz Psychologie Verlags Union.

Krause, U.-M. (2007). Feedback und kooperatives Lernen. Münster: Waxmann.

Moreno, R. (2004). Decreasing Cognitive Load for Novice Students: Effects of Explanatory versus Corrective Feedback in Discovery-Based Multimedia [Electronic Version]. Instructional Science, 32, pp. 99-113.

Narciss, S. (2006). Informatives tutorielles Feedback. Münster: Waxmann.

Nesbit, J. C. \& Adesope, O. O. (2006). Learning with Concept and Knowledge Maps: A Meta-Analysis. Review of Educational Research, 76(3), pp. 413-448.

Novak, J. D. \& Cañas, A. J. (2008). The Theory Underlying Concept Maps and How to Construct and Use Them. [Online]. Verfügbar unter: http://cmap.ihmc.us/ Publications/ResearchPapers/TheoryUnderlyingConceptMaps.pdf [2010-01-10].

Rakoczy, K, Klieme, E, Bürgermeister, A. \& Harks, B. (2008). The Interplay Between Student Evaluation and Instruction [Electronic Version]. Zeitschrift für Psychologie, 216, pp. 111-124.

Ryssel, J. \& Fürstenau, B. (2011). Unterstützung des Lernens betriebswirtschaftlicher Inhalte durch Concept Maps oder Textzusammenfassungen - eine vergleichende Untersuchung im Rahmen des Planspielunterrichts. In: U. Faßhauer, J. Aff. B. Fürstenau \& E. Wuttke (Hrsg.), Lehr-Lernforschung und Professionalisierung : Perspektiven der Berufsbildungsforschung (S.111-121). Opladen

Stark, R., Kopp, V. \& Fischer, M. R. (2009). Förderung der Diagnosekompetenz bei Studierenden der Medizin durch situiertes, fallbasiertes Lernen mit Lösungsbeispielen: der Einfluss von Fehlern und Feedback. Psychologie in Erziehung und Unterricht, 56, 1 S. 37-149. 



\title{
Lehrerausbildung für den berufsbildenden Bereich in Deutschland und England - ausgewählte Ergebnisse einer komparativen Studie
}

\author{
Alexandra Dehmel
}

\section{Einführung}

Knowledge about this key occupational target group (A. D.: staff in technical and vocational education and training), on an international accessible and comparable basis, is extremely scarce. (Maclean \& Wislon 2007: vii)

Lehrerausbildung für den berufsbildenden Bereich wurde bislang kaum aus international-vergleichender Perspektive betrachtet (vgl. Grollmann 2005: 48ff.; Grollmann \& Rauner 2007: xi; Dehmel 2011). Nicht zuletzt ausgelöst durch europäische Entwicklungen (z. B. Bologna-Prozess) und gegenwärtige Reformanstrengungen in vielen Ländern Europas werden aktuell allerdings mehr denn je Forderungen nach vergleichender Forschung laut.

Der vorliegende Beitrag setzt an diesem Defizit an und stellt ausgewählte Ergebnisse einer komparativen Studie zur Lehrerausbildung für den berufsbildenden Bereich in Deutschland und England vor. Betrachtungsgegenstand ist die Ausbildung von Lehrern, die bei den staatlichen Hauptanbietern beruflicher (Erst-)Ausbildung tätig sind, d.h. Lehrer für berufsbildende Schulen in Deutschland und Lehrer für Further Education (FE) colleges in England. Die hier vorgestellten Erkenntnisse wurden im Rahmen einer umfassenden Studie gewonnen (Dehmel 2011), die ein innovatives Forschungsdesign nutzt und nicht nur (1) ein objekttheoretisches Erkenntnisinteresse (Lehrerausbildung), sondern auch (2) ein forschungsmethodologisches Erkenntnisinteresse (methodologische Weiterentwicklung vergleichender Berufsbildungsforschung) verfolgt. Der Schwerpunkt des vorliegenden Beitrages liegt auf (1).

Im Folgenden werden in einem ersten Schritt (Kapitel 2) wesentliche zum Verständnis notwendige - Grundzüge des Forschungsdesigns aufgezeigt. Anschließend (Kapitel 3) werden ausgewählte Ergebnisse vorgestellt, die im Rahmen einer diskursanalytischen Untersuchung von jeweils ca. 50 wissenschaftlichen Publikationen zur Lehrerausbildung aus dem jeweiligen Land gewonnen wurden. Im letzten Teil (Kapitel 4) erfolgen abschließende Bemerkungen. 


\section{Forschungsdesign: Hintergründe und Einblicke}

\subsection{Herausforderungen}

(...) viele Modelle der Lehrerbildung (A. D. scheinen) meinungswie kontextgebundene Konstruktionen, und - wie Popkewitz (1992) scharf formulierte - Ergebnisse von Interessenaushandlungen unterschiedlich starker Lobbies in der "sozialen Arena Lehrerbildung". (Wissenschaftliche) Rationalität kann vielen Modellen der Lehrerbildung nicht immer unterstellt werden. (Buchberger \& Buchberger 2002: 378)

(Lehreraus-)Bildungssysteme sind historisch gewachsene, nationale Lösungen, die in jeweils spezifische, komplexe nationale Kontexte eingebettet sind. Vergleichende Forschung zur Lehrerausbildung erweist sich daher als schwierig, insbesondere wenn mit ihr der (notwendige) Anspruch verfolgt wird, diese Kontexte entsprechend aufzudecken und mit zu berücksichtigen. Auch der vergleichende Forscher selbst geht nicht als sog. ,tabula rasa" ins Forschungsfeld, sondern ist ebenfalls geprägt von seinem eigenen Kontext, vor dessen Hintergrund, aus dessen Blickwinkel heraus er beobachtet, analysiert, interpretiert usw. Hieraus resultiert ein gewisser Ethnozentrismus, der Auswirkungen auf jedes Stadium der Forschung haben und zum sog. Problem der Nostrifizierung, d. h. der „Aneignung des anderen nach eigenem Maß“" (Matthes 1992: 84) führen kann. Georg warnt in diesem Zusammenhang eindringlich:

Die Analyse anderer Kulturen in den Kategorien der eigenen führt notwendigerweise zu Missverständnissen, Artefakten und Fehlinterpretationen, die sich ihrerseits erst durch einen Perspektivwechsel als solche erkennen lassen. (Georg 1997: 158).

Falls dies nicht entsprechend berücksichtigt wird, unterliegt der Vergleich immer der Gefahr, zu einer sogenannten "self-fulfilling prophecy", in der das vorgegebene eine am anderen wiederentdeckt wird" (Matthes 1992: 83), zu werden. Um den soeben aufgezeigten Herausforderungen an vergleichende Forschung entsprechend zu begegnen wurde ein spezielles Forschungsdesign entwickelt und umgesetzt.

\subsection{Grundzüge des Forschungsdesigns}

Zentral ist, dass nicht zu Beginn der Studie eine Festsetzung inhaltlicher Vergleichskriterien i. e. S. erfolgt, sondern das tertium comparationis in Anlehnung an Matthes (1992: 96) als explorativ und sukzessive zu erschließender Denkraum verstanden wird, über dessen Erschließung Vergleichs- 
ergebnisse generiert, kontextuiert und interpretiert werden können. Zur systematischen Erschließung dieses Denkraums wurde ein Forschungsdesign entwickelt, das auf der luhmannschen Beobachtertheorie (Luhmann 1992; 2004) als konzeptionelle Grundlage basiert und die Diskursanalyse im Anschluss an Foucault (Foucault 2007; 2008 u.a.) als methodische Operationalisierungshilfe nutzt. Zur Konkretisierung der forschungsmethodologischen Vorgehensweise erfolgte eine in der qualitativen Sozialforschung verortete Adaption zweier diskursanalytischer Ansätze (Keller 2007a,b; 2008 und Jäger 2004) aus dem Feld der foucaultschen Diskursforschung (vgl. Dehmel 2011: Kap. II, zu einer detaillierten Darstellung des Forschungsdesigns).

Grundidee ist, dass der vergleichende Forscher verschiedene Beobachterrollen einnimmt, und damit der von Georg geforderte Perspektivenwechsel möglich wird (vgl. Dehmel 2009; 2011). Als sog. Beobachter erster Ordnung bleibt er auf Objektebene und betrachtet die Lehrerausbildungssysteme und ihre historischen Entwicklungskontexte. Hieraus resultieren System- und Kontextdarstellungen zu den beiden Ländern. Dabei handelt es sich um deskriptive Beschreibungen der beiden Systeme, die i.d.R. auch klassischer Bestandteil von traditionellen Vergleichsarbeiten sind. ${ }^{1}$ Als sog. Beobachter zweiter Ordnung rücken nationale Beobachter und das was und wie ihrer Beobachtungen zur Lehrerausbildung im eigenen Land in den Blickpunkt. Als Untersuchungsobjekt dienen Diskurse. Konstitutiver Ausgangspunkt ist dabei die Annahme, dass Diskurse die materiale Realisierung von Beobachtungen darstellen, d.h. dass sich die Beobachtungen der nationalen Beobachter in deren Diskursen widerspiegeln, und dass diese über Diskursanalysen erschließbar sind. Die zu untersuchenden Diskurse wurden abgegrenzt über die Kriterien

- Thema: Lehrerausbildung (für berufsbildenden Bereich) in dem jeweiligen Land

- Diskursebene: Wissenschaft

- Publikationszeitraum: 1998-2008

- Datenformat: Textförmige Daten mit Textfunktion Informationstexte

Zur Gewinnung des Materialkorpus wurden verschiedene Strategien und $\mathrm{Zu}-$ gänge genutzt, die national- und kontextspezifisch begründet sind. Der so

1 In der Studie werden die historischen Entwicklungskontexte der Lehrerausbildung nachgezeichnet und Systemdarstellungen vorgenommen. Für die Darstellung des englischen und deutschen Lehrerausbildungssystems wurde dabei auf das Konzept der Qualifizierungsstile von Deißinger (1998) und die darin enthaltenen Dimensionen zurückgegriffen. Dargestellt werden sowohl die ordnungspolitisch-organisatorischen (gesetzliche Grundlagen, zentrale Akteure und ihre Funktionen, System der Steuerung und Qualitätssicherung) als auch die didaktisch-curricularen (curriculare Vorgaben, Qualifikationen, Qualifizierungswege, LehrLern-Gestaltung) Charakteristika der beiden Systeme. 
gewonnene Materialkorpus umfasst pro Land jeweils knapp 50 Beiträge aus wissenschaftlichen Fachzeitschriften und Sammelbänden.

Die vergleichenden Betrachtungen wurden schließlich mit Fokus auf die Ergebnisse der Diskursanalyse vorgenommen und mit Hilfe der System- und Kontextdarstellungen kontextuiert und interpretiert. Der Vergleich der Diskurse erfolgte auf einer inhaltlich-gegenständlichen Ebene (Themen) sowie einer sprachlich-rhetorischen (z. B. Strategien und Mechanismen der Resonanzerzeugung) und formal-strukturellen (z. B.: Zitationskreise, Anstöße für Aussageproduktionen, Positionen und Relationen von Aussageproduzenten und -rezipienten) Ebene und beinhaltet insb. auch die Analyse und den Vergleich von den Diskurs konstituierenden Zusammenhängen (z. B.: über welche sprachlich-rhetorischen Mittel werden bestimmte Themen konstruiert?). ${ }^{2}$ Über den Vergleich konnten wesentliche Gemeinsamkeiten und Unterschiede herausgearbeitet sowie mögliche Begründungs- und Erklärungszusammenhänge offengelegt werden.

\section{Ausgewählte Ergebnisse}

Im Folgenden werden exemplarisch einige ausgewählte Ergebnisse der Studie aufgezeigt. Zu Darstellungszwecken erfolgen die Betrachtungen auf den Ebenen formal-strukturelle und sprachlich-rhetorische Ebene (Kapitel 3.1) sowie inhaltlich-gegenständliche Ebene (Kapitel 3.2), wobei auch auf deren Zusammenhänge eingegangen wird. Des Weiteren werden mögliche Begründungs- und Erklärungsansätze offengelegt sowie Kontextualisierungen vorgenommen.

\subsection{Formal-strukturelle und sprachlich-rhetorische Ebene}

\subsubsection{Virtuelle Textkorpi: Umfang und Struktur}

Auf der Diskursebene Wissenschaft beschäftigen sich die Akteure in Deutschland quantitativ deutlich mehr mit der Lehrerausbildung für den berufsbildenden Bereich als in England. Während in Deutschland ein relativ

2 Die Unterscheidung in diese drei Ebenen dient in erster Linie Analyse- und Darstellungszwecken. Da der Fokus darauf liegt zu ermitteln, welche Themen resp. Inhalte die nationalen wissenschaftlichen Diskurse zur Lehrerausbildung bestimmen, und wie diese hervorgebracht resp. konstituiert werden, schwingt die inhaltliche Perspektive immer mit. 
großer virtueller Textkorpus existiert, ${ }^{3}$ ist er in England vergleichsweise klein. Die dort zu findenden Textbeiträge sind überwiegend in scientific journals veröffentlichte Einzelbeiträge. Im Gegensatz zu Deutschland finden sich kaum Sammelbände oder Beiträge in Sammelbänden, und auch Monographien sind sehr selten.

Begründungsansätze dafür, dass sich die Akteure auf der Diskursebene Wissenschaft in Deutschland deutlich mehr mit der Lehrerausbildung beschäftigen, lassen sich v. a. in den jeweiligen System- und Kontextzusammenhängen beider Länder erkennen. $\mathrm{Zu}$ nennen ist hier insb. die relativ frühe Akademisierung der Lehrerausbildung in Deutschland und die Etablierung der Berufs- und Wirtschaftspädagogik als eigenständige Disziplin im Wissenschafts- und Hochschulsystem, deren Vertreter selbst zentrale Akteure in der Lehrerausbildung sind und diese auch als ihren Forschungs- und Entwicklungsgegenstand definieren. Im Vergleich zu England bestanden in Deutschland somit traditionell andere Notwendigkeiten, aber auch andere Möglichkeiten, sich auf wissenschaftlicher Ebene mit der Lehrerausbildung für den berufsbildenden Bereich zu beschäftigen. In England entwickelte sich keine eigenständige akademische Disziplin für den Bereich berufliche Bildung, und die Beschäftigung mit der Lehrerausbildung für diesen Bereich erfolgte lange Zeit kaum bzw. nur sehr vereinzelt. Da die Lehrerausbildung weder verpflichtend noch vorrangig an den Hochschulen angesiedelt war, bestand hierzu auch keine direkte Notwendigkeit. Erst in den vergangenen Jahren scheint in England - insb. angestoßen durch die Einführung einer verbindlichen Lehrerausbildung im Jahr 2001 (DfEE 2001), aber auch durch eine insgesamt stärkere Fokussierung des berufsbildenden Bereichs i. w. S. langsam ein gewisses Feld zu entstehen.

\subsubsection{Anlässe für Aussageproduktionen: Ausgangs- und Bezugspunkte}

Die Diskurse in den beiden Ländern unterscheiden sich hinsichtlich ihrer Ausgangs- und Bezugspunkte, z. B. hinsichtlich der Anlässe für Aussageproduktionen. In Deutschland sind die Anstöße zu Aussageproduktionen stärker interner Art, d.h. die Diskursakteure stoßen sich selbst gegenseitig untereinander zu Aussageproduktionen an, z. B. im Rahmen von bestimmten Grundsatzfragen. Exemplarisch kann hier auf den diskursprägenden Paradigmenstreit zwischen den Vertretern des berufs(feld)wissenschaftlichen und des fachdidaktischen Ansatzes verwiesen werden, oder auf die Diskussion der Verlagerung von (Teilen der) Berufsschullehrerausbildung von den Uni-

3 „Virtueller Textkorpus“ (Jung 2001: 32, mit Bezugnahme auf Busse \& Teubert 1994) ist ein Begriff, der in der Diskursanalyse verwendet wird und sich hier auf die Gesamtheit des vorfindbaren Materials bezieht, das den für den zu untersuchenden Diskurs definierten Kriterien (s. o.) entspricht. 
versitäten an die Fachhochschulen. Solche internen Anstöße finden sich im englischen Diskurs kaum. Dieser Unterschied ist u. a. in den bereits angesprochenen unterschiedlichen Entwicklungen im Hochschul- und Wissenschaftsbereich begründet. Während in Deutschland eine vergleichsweise große, historisch gewachsene wissenschaftliche community besteht, innerhalb derer solcher Auseinandersetzungen entstehen können und von direkter Relevanz sind (nicht nur bzgl. der Lehrerausbildung, sondern auch bzgl. weitergehender, z. T. existenzieller Fragen wie z. B. der disziplinären Entwicklung der Berufs- und Wirtschaftspädagogik), existierte in England bislang kein derartiges Feld.

Im englischen Diskurs wirken fast ausschließlich bildungspolitische Reformaktivitäten als Auslöser. Der Diskurs wird zur Auseinandersetzung mit den bildungspolitischen Vorgaben der Regierung und deren Reformkonzepten im Kontext der Lehrerausbildung, aber auch im Kontext der Berufsbildung allgemein, genutzt. Auch in Deutschland wirken bildungspolitische Reformaktivitäten (z. B. die Einführung der Bachelor- und Masterstrukturen oder der KMK-Standards für die Lehrerbildung) als Auslöser für Aussageproduktionen. Im Vergleich zu Deutschland ist der englische Diskurs jedoch deutlich stärker durch diese Art von Anstößen geprägt. Ein zentraler Erklärungsansatz hierfür ist, dass ab Mitte der 1990er Jahre eine Vielzahl sehr grundlegender Reformen in der englischen Lehrerausbildung stattfanden (z. B. die Einführung einer Qualifizierungspflicht für Lehrer und entsprechender Vorgaben bzgl. der Gestaltung der Qualifikationen, inkl. verbindlicher Standards und der Implementation eines Akkreditierungs- und Überwachungssystems), d.h. es im englischen Kontext weitaus mehr Anstöße gab als im deutschen.

\subsubsection{Positionierungen: Fremd- und Selbstpositionierungen}

Die soeben genannten Aspekte werden über die Positionierungen verstärkt, die im englischen Diskurs erfolgen. In ihm werden fast ausschließlich Fremdpositionierungen der Regierung vorgenommen. $\mathrm{Zu}$ den zentralen $\mathrm{Zu}-$ schreibungen zählt z. B., dass die Lehrerausbildung als Mittel zum Zweck einer stärkeren Regulierung und Kontrolle des $F E$-Sektors missbraucht wird und den Lehrern eine enge Definition von Professionalität aufgezwängt wird, die an den im FE-Sektor eingeführten Reformen ausgerichtet ist (Stichwörter: accountability, managerialism, performativity etc.). Die Wissenschaft selbst positioniert sich über den Diskurs in erster Linie als Kritiker an den Reformen, macht dabei aber bislang selbst nur in begrenztem Umfang konkrete Vorschläge für andere Ansätze. Sie schreibt sich selbst gravierende Versäumnisse und Defizite in diesem Bereich zu und positioniert sich hierüber als Akteur, der bisher nur wenig selbst aktiv war. Im Gegensatz zum 
deutschen Diskurs erfolgen im englischen Diskurs kaum wissenschaftsinterne Positionierungen, Paradigmenstreits, oder ähnliches, d.h. es gibt keine ausgeprägten Binnendifferenzierungen.

Im deutschen Diskurs sind die Selbstpositionierungen der Wissenschaft deutlich ausgeprägter, d.h. über den Diskurs erfolgen hier quantitativ mehr und auch auf anderem Niveau sowie binnendifferenzierter stattfindende interne Positionierungen. Die Berufs- und Wirtschaftspädagogen präsentieren sich zwar insgesamt durchaus als geschlossene Gruppe mit gemeinsamem Selbstverständnis, aber über die verschiedenen Selbstpositionierungen im Diskurs ergeben sich im Wesentlichen zwei Binnendifferenzierungen: Zum einen zwischen den Wirtschafts- und Berufspädagogen und zum anderen zwischen den Vertretern des fachdidaktischen und des berufsfeldwissenschaftlichen Modells der Lehrerausbildung. Insbesondere aus letztgenannter Differenzierung resultiert ein binnendisziplinärer Paradigmenstreit, in dessen Rahmen über den Diskurs auch unterschiedliche Rollen und Machtpositionen zugeschrieben werden.

Basierend auf den soeben dargestellten Ergebnissen lässt sich insgesamt festhalten, dass es in Deutschland einen ausgeprägten Binnendiskurs in der Wissenschaft gibt, während sich in England erst sehr langsam ein solcher Binnendiskurs entwickelt. Festhalten lässt sich auch, dass die beiden nationalen Diskurse jeweils andere Funktionen innerhalb des jeweiligen Sozialsystems haben.

\subsection{Inhaltlich-gegenständliche Ebene}

Im Rahmen der Diskursanalyse konnte herausgearbeitet werden, dass sich die Themen resp. Inhalte, die den englischen und den deutschen wissenschaftlichen Diskurs zur Lehrerausbildung für den berufsbildenden Bereich im jeweiligen Land bestimmen (bzw. genauer: durch den Diskurs hervorgebracht werden), in weiten Teilen grundlegend unterscheiden. Anders gewendet: in den beiden Ländern werden unterschiedliche Probleme lokalisiert (bzw. zu Problemen gemacht), Schwerpunkte gesetzt, etc. Im Folgenden werden einige Gemeinsamkeiten und Unterschiede aufgezeigt sowie mögliche Erklärungs- und Begründungszusammenhänge offengelegt.

\subsubsection{Charakteristische Bezugspunkte}

Die beiden Diskurse weisen unterschiedliche charakteristische Bezugspunkte auf. Charakteristisch für den deutschen Diskurs ist bspw., dass dort das Wissenschafts- und Hochschulsystem und die disziplinären Entwicklungen der Berufs- und Wirtschaftspädagogik zentrale Bezugspunkte in der Diskussion um die Lehrerausbildung sind. Dies spiegelt sich z. B. im Kontext des bereits 
angesprochenen Paradigmenstreits zwischen den Vertretern des berufsfeldwissenschaftlichen und des fachwissenschaftlichen Ansatzes wieder. Dort werden bspw. unterschiedliche Verständnisse von Wissenschaft, der Aufgabe von Hochschulbildung, und hochschulpolitische Aspekte im Kontext der Lehrerausbildung diskutiert. Anders als im deutschen Diskurs, wird im englischen Diskurs keinerlei Bezug zum Wissenschafts- und Hochschulsystem und damit verbundenen Fragestellungen, z. B. der disziplinären Verortung der an der Lehrerausbildung beteiligten Bereiche oder deren Akzeptanz und Tragfähigkeit, genommen.

Der englische Diskurs weist andere charakteristische Bezugspunkte auf. $\mathrm{Zu}$ nennen ist bspw., dass dort ein relativ großer Fokus auf die Berufsgruppe Lehrer gelegt wird und berufssoziologische Betrachtungen stattfinden. Im Kontext der Lehrerausbildung werden z. B. Aspekte der professionellen Identität, der kollektiven Orientierung an gemeinsamen Standards oder der Handlungsautonomie thematisiert. Im Vergleich dazu wirkt die Berufsgruppe Lehrer im deutschen Diskurs nur selten explizit als Bezugspunkt.

\subsubsection{Qualifizierungsansätze}

Die Diskurse haben gemeinsam, dass es in beiden um Fragen nach der Konzeption von Qualifizierungsansätzen in der Lehrerausbildung geht. Die Aspekte, die hier thematisiert werden, sind jedoch sehr unterschiedlich.

Der englische Diskurs konstituiert sich stark aus der Kritik an der CBET (competency-based education and training)-Ideologie, die den englischen Lehrerausbildungsansatz unterfüttert. Der Fokus liegt fast ausschließlich hierauf. Zentraler Kritikpunkt ist bspw. das hinter dieser Ideologie liegende funktional-behavioristische Kompetenzverständnis, welches als ungeeignet für die Lehrerausbildung beurteilt wird. Die Entwicklung anderer Qualifizierungsansätze wird zwar gefordert, aber eine konkrete Beschäftigung mit alternativen Konzepten erfolgt kaum. Der englische Diskurs bleibt sehr stark in der Kritik an bestehenden Modellen verhaftet.

Der deutsche Diskurs weist hinsichtlich der Qualifizierungsansätze einen deutlich größeren Komplexitätsgrad als der englische Diskurs auf. Er ist dadurch gekennzeichnet, dass er - z. T. auch nur implizit - im Rahmen von zwei historisch gewachsenen, polaren Spannungsfeldern geführt wird.

Dabei handelt es sich zum einen um das (1) Spannungsfeld Lehrer als Fachmann für das jeweilige berufliche Fachgebiet und Lehrer als Pädagoge i.S.e. Fachmanns für Unterricht. Über dieses Spannungsfeld werden Aspekte der Gewichtung und der Integration von fach- und erziehungswissenschaftlichen Anteilen in der Lehrerausbildung thematisiert. Im deutschen Diskurs kommt hier in weiten Teilen die durch ihn mit aufrecht erhaltene, traditionell vorherrschende Idealvorstellung einer bestmöglich abgestimmten Verzahn- 
ung fach- und erziehungswissenschaftlicher Anteile über die gesamte Ausbildungsdauer hinweg zum Tragen. Angestoßen durch die Einführung gestufter Studiengänge wird bspw. die Bedeutung dieser Verzahnung thematisiert. Sie wird auch in der Diskussion um den berufsfeldwissenschaftlichen und den fachdidaktischen Ansatz zum Thema, da bspw. der berufsfeldwissenschaftliche Ansatz genau über die mangelnde Integration begründet wird.

Das zweite Spannungsfeld knüpft an das erste an und ist (2) eine weitere Ausdifferenzierung in die beiden Pole Wissenschaft / Theorie und Praxis. Über dieses Spannungsfeld werden im deutschen Diskurs Aspekte thematisiert, die mit der Frage zusammenhängen, ob die Lehrerausbildung wissenschafts-/theoriebezogen oder praxis-/erfahrungsbezogen erfolgen soll. Dabei werden auch hier wieder Aspekte der Gewichtung und Integration der beiden Pole, aber auch ihrer Funktion thematisiert. Insgesamt betrachtet geht es im deutschen Diskurs hierüber um unterschiedliche Wissensformen sowie ihr Verhältnis zueinander in der Lehrerausbildung und damit verbunden auch unterschiedliche Vermittlungs- und Erwerbsformen dieses Wissens. In Zusammenhang mit der fachwissenschaftlichen Ausbildung kommt z. B. das Verhältnis zwischen dem in den fachwissenschaftlichen Referenzdisziplinen vermitteltem Wissen und dem in der beruflichen Praxis inkorporiertem Wissen i. S. e. Arbeits- und Geschäftsprozesswissens in den Fokus. Während die Vertreter des berufsfeldwissenschaftlichen Ansatzes für speziell zugeschnittene Fachwissenschaften für die Lehrerausbildung plädieren, fordern die Vertreter des fachdidaktischen Ansatzes eine solide fachwissenschaftliche Ausbildung auf dem Niveau der fachwissenschaftlichen Referenzdisziplinen. In Zusammenhang mit der bildungswissenschaftlichen Ausbildung geht es im deutschen Diskurs u. a. um die Einführung von schulischen Praxissemestern in die erste Phase der Lehrerausbildung.

Aus komparativer Perspektive ist es interessant, den englischen Diskurs vor dem Hintergrund der deutschen Spannungsfelder zu betrachten. Dabei stellt man bspw. fest, dass sich in ihm kein Spannungsfeld Fachmann-Pädagoge entfaltet und die Seite Fachmann bislang weitestgehend ausgeblendet blieb. Dies liegt u. a. darin begründet, dass die englische Lehrerausbildung traditionell anders angelegt ist. In ihr findet bislang keine fachwissenschaftliche Ausbildung statt.

\section{Abschließende Bemerkungen}

Im Rahmen des vorliegenden Beitrages kann lediglich ein begrenzter Einblick in die zugrundeliegende Studie geleistet werden. Deutlich wird jedoch, dass das in ihr genutzte Forschungsdesign es ermöglicht, die jeweiligen nationalen Räume und ihre Besonderheiten auf spezifische Weise zu erschließen, 
und somit den zu Beginn aufgezeigten Herausforderungen an vergleichende Forschung zu begegnen.

\section{Literatur}

Buchberger, F. / Buchberger, I. (2002): (Weiter-)Entwicklung der Lehrerinnen- und Lehrerbildung im europäischen Kontext: Impulse aus Finnland und England? In: Beiträge zur Lehrerbildung, 20(3), S. 378-389.

Dehmel, A. (2009): "Ich sehe was, was Du nicht siehst..." - Potenziale der Luhmann'schen Beobachtertheorie für die vergleichende Berufsbildungsforschung. In: Kremer, H.-H. / Sloane, P. F. E. (Hrsg.): Paderborner Forschungs- und Entwicklungswerkstatt. Aktuelle Fragestellungen aus wirtschaftspädagogischen Promotionsprojekten. Band II. Paderborn: Eusl, S. 89-108.

Dehmel, A. (2011): Lehrerausbildung im internationalen Vergleich. Eine diskursanalytische Studie zur Lehrerausbildung für den berufsbildenden Bereich in Deutschland und England. Paderborn: Eusl.

Deißinger, Th. (1998): Beruflichkeit als "organisierendes Prinzip" der deutschen Berufsausbildung. Markt Schwaben: Eusl.

DfEE (2001): Statutory Instrument 2001 No. 1209. The Further Education Teachers' Qualifications (England) Regulations 2001.

Foucault, M. (2007): Die Ordnung des Diskurses. 10. Auflage. Frankfurt am Main: Fischer.

Foucault, M. (2008): Archäologie des Wissens. Köppen, M. (Hrsg.). 14. Auflage Frankfurt am Main: Suhrkamp.

Georg, W. (1997): Zwischen Tradition und Moderne: Berufsbildung im internationalen Vergleich. In: Arnold, R. / Lipsmeier, A. (Hrsg.): Weiterungen der Berufspädagogik. Von der Berufsbildungstheorie zur internationalen Berufsbildung. Festschrift für Antonius Lipsmeier zum 60. Geburtstag. Stuttgart: Steiner, S. $153-166$.

Grollmann, Ph. (2005): Professionelle Realität von Berufspädagogen im internationalen Vergleich. Eine empirische Studie anhand ausgewählter Beispiele aus Dänemark, Deutschland und den USA. Bielefeld: Bertelsmann.

Grollmann, Ph.; Rauner, F. (Hrsg.) (2007): International Perspectives on Teachers and Lectures in Technical and Vocational Education. Dordrecht: Springer.

Jäger, S. (2004): Kritische Diskursanalyse. Eine Einführung. 4. Auflage. Münster: Unrast.

Jung, M. (2001): Diskurshistorische Analyse - eine linguistische Perspektive. In: Keller, R. / Hirseland, A. / Schneider, W. / Viehöver, W. (Hrsg.) 2003: Handbuch Sozialwissenschaftliche Diskursanalyse I. Band 1: Theorien und Methoden. Forschungspraxis, Opladen, S. 29-51.

Keller, R. (2007a): Diskursforschung. Eine Einführung für SozialwissenschaftlerInnen. 3. aktualisierte Auflage. Wiesbaden: VS Verlag für Sozialwissenschaften, GWV Fachverlage GmbH Wiesbaden. 
Keller, R. (2007b): Methoden der sozialwissenschaftlichen Diskursforschung. In: Schützeichel, R. (Hrsg.): Handbuch Wissenssoziologie und Wissensforschung. Konstanz: UVK, S. 214-224.

Keller, R. (2008): Wissenssoziologische Diskursanalyse. Grundlegung eines Forschungsprogramms. 2. Auflage. Wiesbaden: VS Verlag für Sozialwissenschaften, GWV Fachverlage GmbH.

Luhmann, N. (1992): Die Wissenschaft der Gesellschaft. Frankfurt am Main: Suhrkamp.

Luhmann, N. (2004): Einführung in die Systemtheorie. Hrsg. von Dirk Baecker. 2. Aufl. Heidelberg: Carl-Auer-Systeme-Verlag.

Maclean, R.; Wilson, D. N. (2007): Introduction by the Series Editors. In: Grollmann, Ph.; Rauner, F. (Hrsg.): International Perspectives on Teachers and Lecturers in Technical and Vocational Education. Dordrecht: Springer, S. vii-viii.

Matthes, J. (1992): The Operation Called "Vergleichen". In: Matthes, J. (Hrsg.): Zwischen den Kulturen. Die Sozialwissenschaften vor dem Problem des Kulturvergleichs. Göttingen: Schwartz, S. 75-99.

Popkewitz, T. (1992): Changing Patterns of Power. Social Regulation und Teacher Education Reform. New York: SUNY. 



\title{
Triadengespräche zur Rekonstruktion didaktischer Überzeugungen als Bestandteil berufspädagogischer Professionalität
}

\author{
Stephan Kösel
}

\section{Einleitung und Problemstellung}

Dass Überzeugungen als kognitiv-emotionale Dimension neben dem Professionswissen, den motivationalen Grundlagen und der Selbstregulation zum Konstrukt der Lehrerkompetenz und damit der Lehrprofessionalität gezählt werden, ist weitgehend unstrittig (vgl. stellvertretend die Coaktiv-Studien Baumert et al bei Kraus 2011,183). Ob sie dies als Synomym, bzw. Überoder Unterbegriff anderer Konstrukte zur Lehrerkognition tun, wird hingegen sehr kontrovers diskutiert:

Bei Seifried führt eine dezidierte Darstellung unterschiedlicher Überzeugungsansätze, impliziter Persönlichkeitstheorien und subjektiver Theorien $\mathrm{zu}$ der alternativen Begriffswahl der Sichtweisen (2009, 104). Bei Ziegler werden Überzeugungen als kognitive Strukturen der Lehrenden dem Konstrukt der subjektiven Theorien zu- und untergeordnet $(2006,526)$. Ähnlich bei Lehmann-Grube/Nickolaus (2009), die das Lehrerkompetenzmodell von Kraus insofern erweitern, als dass „kognitive Dispositionen auf die Handlungsebene bezogen werden. Subjektive Theorien als Aussagesysteme (...) sich gegenstandsspezifisch über alle Theoriebereiche von den Überzeugungen bis hin zu den handlungsleitenden Kognitionen erstrecken. Ihre Subsumption unter dem Kompetenzaspekt der Überzeugungen ist vor diesem Hintergrund nicht angezeigt." $(2009,62)$ Alich et.al geben wiederum zu bedenken, dass ,subjektive Theorien keine Systeme von Überzeugungen darstellen und im Übrigen auch nicht so gemessen werden (...). Subjektive Theorien (...) können vielmehr situativ mentale Modelle evozieren, die für die subjektive Theorie strukturerhaltende mengenwertige Funktoren von der Theorie aus sind." (Alich et al 2009, 267)

Für die vorliegende Arbeit wird forschungspragmatisch der Überzeugungsbegriff zugrunde gelegt, da er am ehesten über den belief-Begriff an die internationale Forschung anschlussfähig erscheint (vgl. Blömeke et.al 2009, 192). Er soll wie folgt charakterisiert werden.

Als grundlegende Orientierungen zeichnen sich Überzeugungen erfahrungsbasiert durch affektive Aufladung bzw. Wertbindung, eine innere Ordnung mehrerer aufeinander bezogener Vorstellungen aus und verhalten sich 
angesichts lebensgeschichtlich bedingter und subjektiver Prämissen der Weltund Selbstsicht veränderungsresistent. Ihre Funktionalität besteht in einem normativ-evaluativen Charakter, aus dem heraus individuelle Verhaltenssicherheit erwächst (vgl. Reusser/Pauli/Elmer 2010, 480f, Herv. S.K.). Wissenssoziologisch entsteht diese Notwendigkeit nach einem subjektiv fraglosen Bezugspunkt dann, wenn Wissen seine interindividuelle Funktion als Routine verliert und damit Geltungsräume zu Geltungskrisen werden (Oevermann 2006, 102f). Überzeugungen müssen daher auch nicht diskursiv widerspruchsfrei sein. Ihre Plausibilität ist dadurch nicht unbegründet, vielmehr hingegen wertbegründet (vgl. Alisch et.al 2009, 266). Professionstheoretisch relevant sind diese Hinweise insofern, als es danach zum Verständnis der Lehrenden gehört, neben diskursiv-rationalen Logiken des Wissensaufbaus und dessen Anwendung auch identitätstheoretisch-exkludierende Logiken zum Bezugspunkt der Lernprozessplanung und -gestaltung zu machen.

Auf der handlungs- und bewusstseinstheoretischen Ebene der Lernprozesse werden Überzeugungen nicht in einem substitutiven, sondern interdependentem Verhältnis zum (explizitem) Wissen betrachtet. Mit Bezug auf Polanyis Konzept des impliziten Wissens spielen Überzeugungen nach Neuweg eine wichtige Rolle, was im Hintergrundbewusstsein proximal zusammenfindet und dadurch im Fokalbewusstsein distal mit Aufmerksamkeit versehen, d.h. fokussiert werden kann (vgl. Neuweg 1999, 192f). Überzeugungen werden als "Subsidien“ in der impliziten Triade in einer Funktion beansprucht, sie sind nicht Gegenstand unserer Aufmerksamkeit, sondern ihre Instrumente. Das Subjekt achtet von einem oder mehreren Subsiduen auf einen Fokus (Neuweg 1999, 196 Herv. im Org.). Die bewusstseinsfokussierende Funktion von Überzeugungen als Bestandteil mentaler Akte erscheint in einem starken Begriff impliziten Wissens als nicht formalisierbarer Wissensbestandteil, in einem schwachen Begriff impliziten Wissens als ein dem Könner nicht zugänglicher, durch Dritte aber explizierbarer Wissensbestandteil (vgl. Neuweg 2005, 5, Herv. Im Org.).

Welche Forschungsfragestellungen ergeben sich aus dem bisher Skizziertem?

Werden Überzeugungen als Bestandteil einer Wissensbasis verstanden, mit der Lehrprofessionalität realisiert werden kann, so stellt sich die Frage, ob und, wenn ja, wie man auf Überzeugungen im Rahmen der Lehrerbildungsphasen als Aspekt des Professionalisierungsprozesses eingehen kann und soll. Und damit ihre Genese, ihre Analyse bzw. ihre potentielle bewusstseinsfokussierende Funktionalität zum Thema gemacht wird. Der vorliegende Beitrag bejaht eindeutig diese Frage, da über die Problematisierung des Überzeugungskonstruktes eine zentrale Fragestellung der Lehrerbildung in den Blick gerät, die sonst erst, nur und zwangsläufig unter ganz anderen Vorzeichen in der zweiten Lehrerbildungsphase bearbeitet würde: Das Verhältnis von Lehrerwissen und Lehrerkönnen aus biographisch-evolutionärer Sicht. 
Eine zweite zentrale Fragestellung ergibt sich aus der Tatsache, dass in Überzeugungskonzeptionen in der Regel der Variable der Lehrerüberzeugungen die dominante Rolle nicht nur in Planung und Reflexion, sondern auch in der Durchführung des Lerngeschehens zugewiesen wird. In der Lehrerbildungs- und Lehrerkognitionsforschung gibt es eine umfangreiche Befundlage, über den Einfluss von unterrichtsbezogenen Überzeugungskonzeptionen im Sinne einer Wirkungskette von Lehrerüberzeugung, Lernprozesse und Lernzuwächsen (ausführlich bei Seifried 2009, 30ff.). Die Lernerüberzeugungen hingegen werden selten als eigenständige Variable konzeptualisiert. Es verwundert daher nicht, dass darüber hinaus das Aufeinandertreffen von konvergenten, divergenten oder unterschiedlich dimensionalisierten Lehrer- und Lernerüberzeugungen im Unterrichtsprozess als systemisch-rekursive Überzeugungskonstellationen bisher überhaupt nicht als Forschungsgegenstand von Unterrichtsdynamik problematisiert wurde: weder in der Unterrichtsforschung noch als zu reflektierendes bzw. zu theoretisierendes Phänomen in der Lehrerbildung.

Als dritte Fragestellung ergibt sich aus den beiden ersten die Rolle und Funktion der Didaktik, als Wissensbestandteil des Lehrerwissens und als Handlungskomponente des Lehrerkönnens. Im vorliegenden Beitrag wird davon ausgegangen, dass Didaktik zu der von Shulman (1986) und Bromme (1992) (bei Neuweg 2010, 454) wesentlich mitgeprägten Dimensionierung des Lehrerwissens in das Fachwissen (Inhaltswissen, wissenschaftstheoretisches Wissen, Philosophie des Faches), fachdidaktisches Wissen (Pädagogisches Inhaltswissen, curriculares Wissen) und fachindifferentes Wissen (Wissen über Schultheorie, Berufsbildungstheorie, Gesellschaftstheorie), konstitutive Bezüge aufweist.

So hängt es je nach didaktischer Theoriebildung wesentlich davon ab, in welchen der drei klassischen didaktischen Dimensionen der Inhalte (Sache), der Akteure (Ich) und der gruppendynamisch bedingten Lernprozesse (Wir) es (un-)wahrscheinlich zu solchen Überzeugungskonstellationen kommt. Wenn didaktische Theoriebildung etwa nicht klärt, worin der Transformationsprozess zwischen Objekt und didaktisiertem Gegenstand, bzw. der Unterschied zwischen der Erschließungsfähigkeit des Subjektes und der Erschließbarkeit des didaktisierten Gegenstandes besteht (vgl. kritisch dazu Gruschka 2002), spricht dies etwa bezogen auf das fachdidaktische Wissen ganz spezifische Überzeugungen über typische Aneignungsschwierigkeiten von Lernern an. Formal ähnlich, qualitativ anders überzeugungsinduzierend kann es auf das Lehrerwissen wirken, wenn man nicht die bildungstheoretische Frage der Inhalte als paradigmatischen Ausgangspunkt für didaktisches Denken wählt, sondern eine lern(prozess)theoretische, erzieherische oder gar institutionell-systemische Perspektive (vgl. ausführlich dazu Terhart 2009, 107ff). Aus berufspädagogischer Sicht veranschaulichen die Diskussionen um die curricularen Konstruktionsprinzipien des Lernfeld- 
konzeptes (jüngst Tramm 2011 in Replik auf Reinisch), dass je nach Wissenschafts- bzw. Situationsorientierung z.B. die „Philosophien“ bzw. Überzeugungen bezogen auf die Rolle der Unterrichtsfächer unterschiedlich aktiviert bzw. tangiert werden.

Weiterführend ist zu fragen, wo und wie solche Überzeugungskonstellationen sogar als Ergebnis didaktischer Planung oder situativ-didaktischer Handlungskompetenz intentional gewollt, zugelassen bzw. induziert werden? Welche unterrichtlichen Formen können den potentiellen Anteil von Überzeugungen am Aufbau von implizitem und explizitem Wissen vor und nach Unterrichtshandlungen kommunizierbar machen? Wird dies nicht ermöglicht, trivialisiert dies zwangsläufig die anzustrebende Expertise bzw. reduziert sich berufliche Didaktik hierbei auf die Förderung von bloßer Intuition.

Berufsbildungstheoretisch war die Aneignungsfrage immer eng mit der Anwendungsfrage verknüpft, die allgemeindidaktische Fragestellung insofern um einen direkten betrieblichen (Duale Ausbildung) oder/und zukünftigen (Übergangssystem, Betriebswechsel) Handlungskontext erweitert, so dass eine berufliche Didaktik sich schon im Unterricht mit einer spezifisch anderen, nämlich verdoppelten, rekursiven Handlungsdynamik auseinandersetzen muss. Mit der unterrichtlichen Handlungsdynamik und mit der zu bewältigenden, didaktisierten beruflichen Handlungsdynamik. Mit dem einen Bildungsziel der beruflichen Handlungskompetenz bzw. der Lernfeldorientierung hat es eine berufliche Didaktik insofern mit der Verschränkung unterschiedlicher Erzeugungsmodi von gelingender Praxis (Wissen und Können) zu tun. Eine solche Verschränkung erfolgt zum einen lernfeldorientiert während des Unterrichts mehrfach-rekursiv und zum anderen in Bezug auf zwangsläufig individualisierte Kompetenzentwicklungsprozesse (vgl. Tramm 2011,11), die wiederum überzeugungsinduzierend wirken können müssen. Insofern stellt es eine konstitutive Antinomie beruflicher Didaktik in dualen Ausbildungsklassen dar, den Unterricht über Lernsituation zu planen und ihn gleichzeitig individuell lernsituativ zuzulassen. Angesichts der Herausforderung für die Auszubildenden im Rahmen der Lernortkooperation zwei Lernkontexte mit unterschiedlichen Systemrationalitäten von Bildungs- und Verwertungslogiken (ausführlich Kösel 2005) aufeinander beziehen zu müssen, wird nämlich zwangsläufig der normativ-evaluative Charakter von Überzeugungen aktiviert bzw. werden spezifisch individualisierte Synchronisationsleistungen erforderlich. Ähnliches ergibt sich auf Einzellehrerseite für die im Rahmen der curricularen Konstruktionsarbeit der Lernsituationen nötige Lehrerteamarbeit bzw. die damit verbundenen Synchronisationsleistungen unterschiedlicher Philosophien der Fächer.

Nimmt man das andere Bildungsziel, das der Subjektbildung, hinzu, wirken die voran erwähnten exkludierenden-identitätstheoretischen Logiken ebenso überzeugungsinduzierend. 
Die aufgeworfenen Fragestellungen können und sollen im vorliegenden Beitrag nicht alle umfassend behandelt werden, was an anderer Stelle in systematischer Form erfolgen wird (Kösel 2012). An ausgesuchten Aspekten eines universitären Projektes der Hochschuldidaktik werde ich jedoch veranschaulichen, dass schon während der ersten Phase der Lehrerbildung auf individueller Ebene der Studierenden Dimensionen eines weiten Verständnisses von didaktischen Überzeugungen rekonstruiert werden können. Weit im beschriebenen Sinne in der Rekonstruktion impliziter didaktischer Theoriebestandteile, die sich aus eigener betrieblicher Ausbildung bzw. aus vorherigen Studien- und Berufserfahrungen gebildet haben und nun im Rahmen des Hochschulstudiums als „,biographischer Rucksack“ sowohl mit didaktischem Wissen als auch mit den anderen Lehrerwissensbereichen $\mathrm{zu}$ einer individuellen Wissensbasis verbunden werden müssen. Für ein enges Verständnis von didaktischen Überzeugungen auf unterrichtlicher Handlungsebene können diese Rekonstruktionen auf potentielle Überzeugungskonstellationen hinweisen, die aufgrund der weiten didaktischen Überzeugung für den Einzelnen auf der späteren Handlungsebene relevant werden könnten. Eine (theoretische) Modellierung potentiellen didaktischen Wissens bzw. didaktischer Überzeugungen für den prozessuralen Umgang mit solchen Überzeugungskonstellationen kann und soll an dieser Stelle nicht geleistet werden.

\section{Berufsbiographische Rekonstruktionen bei Lehramtsstudierenden als Forschungskontext}

Für ein Gesamtkonzept der Lehrerbildung hat Neuweg ,zwölf Figuren der Relationierung von Lehrerwissen und Lehrerkönnen“" unterschieden, die jeweils einem Integrations- versus Differenzierungskonzept von Wissen und Können bzw. als Folge davon den verschiedenen Phasen zugeordnet werden können (Neuweg 2010, 36).

Da wir das Projekt MasterNet im Jahr 2011 auf Studierende der TU Darmstadt bezogen haben, die nach einem vorherigen Studium und Arbeitserfahrungen direkt in das Masterstudium quer einsteigen, kann zwischen der Figur Brille, der Induktion und der Persönlichkeit ein spezifisches Spannungsverhältnis entstehen, was für den Forschungskontext der Überzeugungen besonders interessant erscheint. Die Figur der Brille steht für eine systematische Vermittlung von berufspädagogisch relevantem Orientierungsund Reflexionswissen, in der Einführung in spezifische Begriffswelten, Problemmodelle und Deutungssysteme. Die Figur der Induktion stellt die Erfahrung und Praxis vor die Theorie. In unserem Falle soll ,die systematische Konfrontation der objektiven Theorien“ (Neuweg 2010,37) mit den umfang- 
reichen Ausbildungs-, Studien- und Arbeitserfahrungen nicht zu der Frage führen, ob die Figur der Persönlichkeit für den Lehrerberuf geeignet ist, sondern wie zu Studienbeginn schon mit relevanten Überzeugungsdimensionen evolutionär-persönlichkeitsbildend umgegangen werden kann.

Der Forschungsansatz besteht darin, dies mit der aus dem Wissensmanagement heraus von Dick entwickelten narrativen Methode der Triadengespräche zu realisieren. Hierbei stammen die zwei Rollen des Experten und Novizen aus demselben Handlungskontext (Studieneinstieg mit beruflichen Vorerfahrungen), zeichnen sich jedoch durch unterschiedliche biographische Stationen jeweils als Experten aus. Die ansonsten in den klassisch dyadischnarrativen Interviewformen konstitutive Forscherrolle geht in der des Laien als methodischem Zuhörer in der Triade auf.

„Durch die beiden Zuhörer werden an den Erzähler unterschiedliche Anforderungen gestellt: Während für den fachlichen Zuhörer (Novizen) die Relevanz und Nützlichkeit des Erzählten bedeutsam ist, ist für den Laien dessen Verständlichkeit von Bedeutung" ( Dick 2006, 146.).

Die Biographieforschung basiert u.a. darauf, ,den Standpunkt des biographischen Subjektes einzunehmen und den Prozess als eine vom Individuum zu lösende Aufgabe zu begreifen" (Schulze 2010, 427). Narrationen werden als ursprünglicher Modus der Erfahrungsorganisation verstanden, „die einen gemeinsamen und abgrenzbaren Sinnzusammenhang - das Narrativ- bilden. (...) In der Erzählung verschränken sich das Nacherleben des Ereignisstromes mit der reflexiven Konstruktion und Perspektivenvariation. (vgl. Dick 2006, 143f). Durch die Triadenmethode war insofern beabsichtigt, relevante berufsbiographische Konstruktionsdimensionen des jeweiligen Experten induktiv berichtbar werden zu lassen, die sich implizit auf didaktische Theoriebestandteile beziehen und insofern den „brillenförmigen“ Wissensaufbau mit beeinflussen. Die in der Triadenmethode geschulten Projektmitarbeiter hatten entlang der drei Erzählimpulse „Ausbildungserfahrung“, „Studien- und Berufserfahrungen“ und „Lehrerselbstverständnis“ lediglich die Aufgabe durch Rück- oder Gegenfragen den narrativen Erzähl- und Gesprächsfluss zwischen Experten und Novizen aufrecht zu erhalten. Die Triadengespräche dauerten 50-60 Min und wurden bisher mit 27 Studierenden durchgeführt.

\section{Erste Ergebnisse und Typenbildung}

Die Triadengespräche wurden transkribiert und nach der induktiven grounded theory-Methode (Strauss 1998) zunächst offen, dann axial kodiert. Gemäß den Aspekten des Kodierparadigmas - Bedingungen, Interaktionen, Strategien, Konsequenzen - (vgl. dazu Hülst 2010, 288) wurden anschließend 
die Interviews daraufhin analysiert, welches berufsbiographisch relevante Zentralmotiv und welche darauf bezogenen Unterkategorien sich rekonstruieren lassen und dadurch spezifische Bezüge zu didaktischen Dimensionen abgeleitet werden können.

Obwohl die Erfahrungsaufschichtung (Schütze 1984, Schulze 2010, 418ff.) der Triadengespräche methodologisch bedingt hinter denen autobiographisch-narrativer Interviews zurückfallen muss, haben wir dennoch in einem zweiten methodischem Zugriff die „kognitiven Figuren des Stehgreiferzählens" nach Schütze (1984) als Interpretationsbezugspunkt des jeweiligen Zentralmotivs verwendet. Die ,grundsätzlichen Haltungen gegenüber lebensgeschichtlichen Erlebnissen“" (Schütze 1984, 92) - biographische Handlungsschemata, institutionelle Ablaufmuster, Verlaufskurven und Wandlungsprozesse - beziehen sich als Muster der Erfahrungsaufschichtung insofern auf die aus den Triadengesprächen identifizierbaren Prozesse bzw. Segmente der berufsbiographischen Schilderungen.

\subsection{Lehramtsstudentin,,Claudia“ als Typus einer Sachverwalterin}

Für Studentin „Claudia“ lässt sich ein biographisches Handlungsmuster als „übergreifende Form der Verknüpfung von Ereignissen und Erfahrungen (Schütze 1984,93) rekonstruieren. Das Zentralmotiv „Ich will kreativ sein“ bezieht sich als berufsbiographische Haltung sowohl auf die absolvierte Ausbildung als Mediengestalterin, dem abgeschlossenem Design-Studium und dem zukünftigen Berufs- und Lehrerbild für berufsbildende Schulen. Als biographisches Handlungsmuster kommt es auf diesen Ebenen zu einer Fokussierung der eigenen Spontaneitäts- und Aktivitätssphäre gegenüber institutionellen Ablaufmustern als gegensätzliche kognitive Figur, die als externe Erwartungsfahrpläne eine notwendige Ich-Anpassung erfordern. Die Zukunftserwartungen von Claudia richten sich daher eher an selbst zu gestaltende Realisierungsräume, in denen sich das Ich vom kollektiven Wir abgrenzen kann (vgl. Schütze 1984, 93f).

Bezogen auf die Dimensionen des Lehrerwissens lässt sich eine dazu passend sehr ausgeprägte „Philosophie des Berufsfeldes“ rekonstruieren, bei den Auszubildenden primär und besonders die Kreativität zu fördern (,nicht nur Ausführer im Betrieb") und Curriculumvorgaben eher als Einschränkung zu verstehen (,muss mal sehen, was ich da dran drehen kann“).

Bezogen auf die Meso-Ebene der Lernortkooperation wird eine explizite Wächter- und Kompensationsfunktion als Lehrerin deutlich. Einerseits wird Berufsschule als Schon- und Übergangsraum in den Übertritt in das Arbeitsleben definiert ("dass das nicht so `n krasser Cut ist"), gleichzeitig werden an die Auszubildenden passend zum eigenen biographischen Handlungsmuster 
implizite Regressionserlaubnisse gegenüber institutionellen Erwartungen erteilt (,dürfen einmal in der Woche in die alte Rolle des Schülers").

Gemäß dem Kodierparadigma nach Strauss kann man für „Claudia“ nach den Bedingungen fragen, die auf den didaktischen Ebenen gegeben sein müssten, damit das Entstehen bzw. Zulassen von individuellen Entfaltungsräumen als zentrales Ziel von „Claudia“ realisiert werden kann. Die dazu nötigen Interaktionen sind daher eher als Abgrenzungsverhandlung, als Zugeständnis partieller Interessen zu erwarten. Die entsprechende Strategie wird eher zu Teilsystem- und Nischenbildung führen. Als Konsequenz ergibt sich für den Umgang mit den Antinomien des Lehrerhandelns (z.B. Macht vs. Autonomie, Organisation vs. kommunikative Rückkoppelung, vgl. Helsper 2004) eher die Entscheidung für eine der Antinomieseite, statt ein zu suchendes, jeweils situatives Gleichgewicht (vgl. Schütze 1996, 137f).

„Claudia“ kann im Sinne der Typenbildung als Fall gewertet werden, für die ein biographisches Handlungsmuster nicht nur den wesentlichen Ausgangspunkt für ein Lehrerbild als „Sachverwalterin einer Berufsfeldphilosophie" darstellt, sondern auch die Relation zwischen Wissen und Können durch die Ermöglichung von möglichst hohen Freiheitsgraden mitbestimmt. In einem weiten Verständnis didaktischer Überzeugungen ist insofern im Rahmen der universitäten Phase zu thematisieren, inwieweit die didaktische Theoriebildung für „Claudia“ bisherige Überzeugungen anspricht, erweitert bzw. konstruktiv irritieren kann.

\subsection{Lehramtsstudent,, Simon" als Kontrastfall und Typus des Interaktionisten}

Der Kontrastfall „Simon“ kann hingegen in einer Typenbildung als „Interaktionist" bezeichnet werden, dessen Zentralmotiv die kognitive Figur des Wandlungsprozesses darstellt. Als Wendepunkt einer sich anbahnenden „dynamische Entfaltung innerer Prozesse, als aufzulösende Diskrepanz zwischen Aktivitätsplanung und Handlungsrealisation“ (Schütze 1984) bilanziert „Simon" nach einer Schreinerlehre als Inhabersohn, einem Bauingenieurstudium und Arbeitstätigkeit, sich erst vor der Studienentscheidung für das Lehramt gefragt zu haben: "Was passt eigentlich wirklich zu mir?“. Institutionelle Ablaufmuster haben für ihn weniger einschränkende, vielmehr konstruktive Überprüfungsfunktion, externe Erwartungsfahrpläne dienen als Kompass für das eigene berufsbiographische Suchmotiv.

Aus einer empathischen Grundhaltung heraus erwächst so ein interaktionistisches Lernortverständnis mit ausdifferenzierten Gelingensbeding-

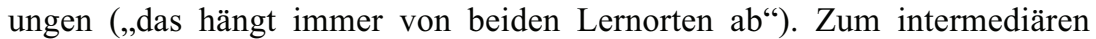
Lehrerbild gehört eine Beraterfunktion für jugendtypische Wandlungsprozesse (,da hat man definitiv andere Sachen im Kopf") und die Ausbildungszeit als Einsozialisation in Erwartungsfahrpläne (,,da herrschte halt ein 
Kasernenton“). Für ein integrierendes Lehrerbild sind für „Simon“ die unterschiedlichen Rationalitäts- und Habitusformen als fachkompetenter Praktiker (,Wer nicht Bescheid weiß, ist bei den Azubis unten durch“), als situationsgerechter Vermittler („Man muss die Fachsachen schon rüberbringen können und nen Bezug zu den Schülern haben") bekannt. Sie stehen für ihn als Wandlungsprozess im Rahmen der eigenen Professionalisierung im Raum (,wie man das hinbekommt").

Für „Simon“" stellt sich als „Interaktionist" bezogen auf die Bedingungen didaktischen Handelns insofern eher die Frage nach einem gleichberechtigten Austausch als individuellem Suchprozess. Der Strategie des Kompromisses entstammen eher verstehend-offene, statt wie bei „Claudia“ sich abgrenzende Interaktionen. Als Konsequenz wird „Simon“" eher anspruchsvolle Synchronisationsleistungen in antinomischen Handlungssituationen von sich abverlangen, da er sich der paradoxalen Struktur eher bewusst ist (vgl. Schütze 1999, 252ff).

In einem weiten Verständnis didaktischer Überzeugungen ist bei „Simon" im Rahmen der universitären Phase zu thematisieren, wie die didaktische Theoriebildung dafür sorgen kann, wann und in welchen Handlungsanforderungen eher die „Kultur des Einlassens“ und damit interaktionistisches, verstehend-situatives Überzeugungshandeln bzw. wann eher die „Kultur der Distanz“ (vgl. Neuweg 2005, 7ff) und damit die einer fallentlasteten Routineanwendung sinnvoll erscheint und „Simon“ vor potentieller Überforderung geschützt werden kann.

\section{Weiterführende theoretische Überlegungen und Ausblick}

Die Typenbildung für die Gesamtgruppe der Studierenden erfolgt zum Projektabschluss im Sommer 2012. Mit den Triadengesprächen kann methodisch abgesichert für die Relationierung von Lehrerwissen und Lehrerkönnen ein individuelles, biographisch relevantes und in der Regel ausgeblendetes Erfahrungspotential kommunizierbar und für eine personalisierte Professionalisierungsphase anschlussfähig gemacht werden. Durch die Narrations- und Explikationsprozesse auf Augenhöhe mit dem Novizen kann Selbstverständliches, unhinterfragt Erlebtes in eine elaborierte Beziehung zum universitären Wissensaufbau und den Reflexionsprozessen gestellt werden. So besuchte jeder Teilnehmer im darauffolgenden Studiensemester eine Seminarveranstaltung mit einer personalisierten Entwicklungsaufgabe (vgl. Hericks 2006), die sich explizit auf die rekonstruierte, berufsbiographische Zentraldimension und einen didaktischen Theoriebestandteil bezog. „Claudia“ beschäftigte sich mit Bildungsgängen des Übergangsbereiches, bei denen ihre ausgeprägte „Philosophie des Berufsfeldes“ zu kritischen Überzeugungskonstellationen 
mit den dortigen Adressaten führen kann. „Simon“ thematisierte im Rahmen eines Curriculumseminars wie er sein berufliches Können mit Lehrinhaltswissen $\mathrm{zu}$ einer klaren fachdidaktischen Haltung verbinden kann, die dennoch im Unterrichtsprozess Überzeugungskonstellationen aus Lernersicht zulassen kann.

Für eine Konzeptionierung didaktischer Überzeugungen können und sollen mit diesem Ansatz keine Wirkungsanalysen für den Umgang mit Überzeugungskonstellationen angestrebt werden. Vielmehr weisen die Ergebnisse darauf hin, dass sich situiert-intuitives Lehrerhandeln nicht als bloßes Ergebnis spontaner Einlassung oder als Einübung von Routinen in der zweiten Phase darstellt, sondern schon universitär die Spezifika und individuelle Funktionalität von Überzeugungen für solch kontextsensibles spätere Verhalten theoretisiert und problematisiert werden können.

Eine Konzeptionierung didaktischer Überzeugungen in der beruflichen Bildung wird sich neben der Lehrerprofession mit der Professionalisierung des betrieblichen Ausbildungspersonals zu beschäftigen haben. Hierbei wird $\mathrm{zu}$ untersuchen sein, inwieweit gerade dort wirksame, andere Professionalisierungs- und Handlungslogiken spezifisch andere Überzeugungskonstellationen generieren, wenn es etwa um Wissensbestände des Arbeitsprozessbzw. Erfahrungswissen geht.

\section{Literatur}

Alisch, L/Hermkes, R./Möbius, K. (2009): Messen von Lehrprofessionalität II: Metrologie. In: Zlatkin-Troitschanskaia/Beck/Sembill/Nickolaus, /Mulder, R.(Hrsg) (2009): Lehrprofessionalität. Bedingungen, Genese, Wirkungen und ihre Messung. Weinheim. S. 263-274.

Blömeke, S./Kariser, G./Lehmann, R/König J. (2009): TEDS-M: Messung von Lehrerkompetenzen im internationalen Vergleich. In: Zlatkin-Troitschanskaia/ Beck et al. (Hrsg) (2009): Lehrprofessionalität. Bedingungen, Genese, Wirkungen und ihre Messung. Weinheim. S. 181-210.

Dick, M. (2006): Triadengespräche als Methode der Wissenstransformation in Organisationen. In V. Luif, G./ Thoma \& B. Boothe (Hrsg.): Beschreiben - Erschliessen - Erläutern. Psychotherapieforschung als qualitative Wissenschaft. Lengerich. S. 141-166.

Gruschka, A: (2002): Didaktik. Das Kreuz mit der Vermittlung. Elf Einsprüche gegen den didaktischen Betrieb. Wetzlar.

Helsper, W. (2004): Antinomien. Widersprüche. Paradoxien - Lehrerarbeit - ein unmögliches Geschäft In: Koch-Priewe /Kolbe /Wildt: Grundlagenforschung und mikrodidaktische Reformansätze der Lehrerbildung. Bad Heilbrunn/Obb. S. 4998.

Hericks, U. (2006): Professionalisierung als Entwicklungsaufgabe. Wiesbaden. 
Hülst, D (2010): Grounded Theory. In: Friebertshäuser, B./ Langer, A.,/Prengel, A. (Hrsg.): Handbuch qualitative Forschungsmethoden in der Erziehungswissenschaft. Weinheim. S. 281-300.

Kösel, S. (2005): Ist die Berufsschule noch zukunftsfähig?. Eine systemtheoretische Analyse der Dualen Lernorte in der Wissensgesellschaft. Bielefeld.

Kösel (2012) in Vorb.: Das Forschungsprogramm didaktischer Überzeugungen als Bestandteil berufspädagogischer Professionalität.

Kraus, S. (2010): Das Experten-Paradigma in der Lehrerforschung, In: ZlatkinTroitschanskaia/Beck et al. (Hrsg) (2009): Lehrprofessionalität. Bedingungen, Genese, Wirkungen und ihre Messung. Weinheim. S. 171-191.

Lehmann-Grube, S. /Nickolaus, R. (2009): Professionalität als kognitive Disposition. In: In: Zlatkin-Troitschanskaia /Beck et al., (Hrsg) (2009): Lehrprofessionalität. Bedingungen, Genese, Wirkungen und ihre Messung. Weinheim. S. 59-70.

Neuweg, G.H. (1999): Könnerschaft und implizites Wissen. Zur lehr-lerntheoretischen Bedeutung der Erkenntnis- und Wissenstheorie Michael Polnyis. Münster.

Neuweg, G.H. (2005): Emergenzbedingungen pädagogischer Könnerschaft. In Heid, H./Harteis C.(Hrsg.): Verwertbarkeit. Ein Qualifikationskriterium (erziehungs-) wissenschaftlichen Wissens?. Wiesbaden. S. 205-228.

Neuweg, G.H. (2010): Fortbildung im Kontext eines phasenübergreifenden Konzeptes zur Lehrerbildung. In: Müller, F. (Hrsg.) (2010): Lehrerinnen und Lehrer lernen. Münster. S. 35-50.

Neuweg, G.H. (2011): Das Wissen der Wissensvermittler. Problemstellungen, Befunde und Perspektiven der Forschung zum Lehrerwissen. In: Terhart E., Bennewitz, H./ Rothland, M. (Hrsg.): Handbuch der Forschung zum Lehrerberuf. Münster. S. 451-477.

Oevermann, U. (1999): Theoretische Skizze einer revidierten Theorie professionalisierten Handelns. In: Combe, A./Helsper, W. (Hrsg.) (1999): Pädagogische Professionalität. Untersuchungen zum Typus pädagogischen Handelns. S. $70-182$

Oevermann, U. (2006): Wissen-Glauben-Überzeugung. Ein Vorschlag zu einer Theorie des Wissens in krisentheoretischer Perspektive. In : Tänzler, D, et al. (Hrsg.): Neue Perspektiven der Wissenssoziologie. Konstanz. S. 79-118.

Reusser, K./Pauli, P./Elmer, E. (2011): Berufsbezogene Überzeugungen von Lehrerinnen und Lehrern. In: Terhart E/Bennewitz,H./ Rothland, M.(Hrsg.): Handbuch der Forschung zum Lehrerberuf. Waxmann-Verlag. Münster. S. 487-496.

Schütze, F. (1984): Kognitive Strukturen autobiographischen Stehgreiferzählens. In: Kohli, M./Robert, G. (Hrsg). Biographie und soziale Wirklichkeit. Neue Beiträge und Forschungsperspektiven. Stuttgart. S. 78-117.

Schütze, F. (1999): Organisationszwänge und hoheitstaatliche Rahmenbedingungen im Sozialwesen. Ihre Auswirkungen auf die Paradoxien des professionellen Handelns. In: Combe, A./Helsper, W. (Hrsg.) (1999): Pädagogische Professionalität. Untersuchungen zum Typus pädagogischen Handelns. Frankfurt a.M.

Schulze, T. (2010): Zur Interpretation autobiographischer Texte in der erziehungswissenschaftlicher Biographieforschung. In: Friebertshäuser, B., Langer, A.,/Prengel, A. (Hrsg.): Handbuch qualitative Forschungsmethoden in der Erziehungswissenschaft. Weinheim. S. 413-436. 
Seifried, J. (2009): Unterricht aus Sicht von Handelslehrern. Konzepte des Lehrens und Lernens. Band 16. Frankfurt a.M.

Strauss, A. L. (1998): Grundlagen qualitativer Sozialforschung. UTB-Taschenbuch. München.

Terhart, E. (2009): Didaktik. Eine Einführung. Stuttgart.

Tramm, T. (2011): Ist das Glas nun halbvoll oder halbleer?. Ein Beitrag zur berufsund wirtschaftspädagogischen Diskussion des Lernfeldkonzeptes. In: bwp@ Berufs- und Wirtschaftspädagogik-online. Ausgabe 20, 1-22. online: http://www. bwpat.de/ausgabe20/tramm_bwpat20.pdf (27-06-2011).

Ziegler, B. (2006): Subjektive Theorien und didaktisches Handeln. Forschungsaktivitäten und Befunde zu Lehrenden in der beruflichen Bildung. In: Zeitschrift für Berufs- und Wirtschaftspädagogik (ZBW) Heft 102, S. 525-551. 


\section{Entwicklung eines Instruments zur Erfassung unterrichtsbezogener Metaphern ${ }^{1}$}

Birgit Lehmann

\section{Hintergrund und Stand der Forschung}

\section{Problemstellung}

Lehrpersonen haben die Aufgabe, in verschiedenen Bildungsgängen unter Berücksichtigung der jeweiligen curricularen Vorgaben das „Produkt" professionelle - d.h. auf ausgewiesenen Standards basierende - Lernumgebungen zu entwickeln (Ebner, 2010). Dieses „Produkt" repräsentiert gemäß dem Angebot-Nutzungsmodell nach Fend (2002) - ein different gestaltetes, mehr oder weniger adäquates Angebot, das von Schülerinnen und Schülern zum Aufbau (beruflicher) Handlungskompetenz genutzt werden kann (Ebner, 2010). Die reliable und valide Erfassung von ,Lehrerwissen " bezüglich des Zusammenfügens unterrichtlicher Phänomene („Orchestrierung") lässt erwarten, dass es hierzu erforderlich ist, eine Vielzahl an Instrumenten einzusetzen. Eine verhältnismäßig ökonomische Vorgehensweise bietet die Nutzung von Konstrukten, für die postuliert wird, dass sie die Orchestrierung steuern. Dieser Arbeit liegt die Annahme zugrunde, dass eine substanzielle Beziehung zwischen der Planung und Realisierung von Lernumgebungen $^{2}$ [Variable 2] und jenen Metaphern [Variable 1] besteht, die von (angehenden) Lehrpersonen zur Umschreibung der Lehrertätigkeit verwendet bzw. dazu für geeignet befunden werden. Beispielsweise könnten Lehrpersonen, die Metaphern der Kategorie Berater ${ }^{3}$ für treffender halten, mehr Zeit im Unterricht für beratungsaffine Tätigkeiten aufwenden und möglicherweise eine andere Komposition von Unterrichtsmerkmalen wählen (Ebner \& Lehmann, 2012) (siehe Abb. 1).

1 Der Beitrag wurde im Rahmen des „Young Reseachers-Programms“ mit dem 1. Preis ausgezeichnet. Meinen herzlichen Dank möchte ich dem Betreuer meines Dissertationsvorhabens Herrn Prof. Dr. Hermann G. Ebner, allen Teammitgliedern der Mannheimer Area „Wirtschaftspädagogik“, der Jury des „Young Reseachers-Programms“, der Stiftung für Wissenschaft und Gesellschaft der Universität Konstanz sowie dem Organisatorenteam aussprechen.

2 Im Rahmen dieser Qualifikationsarbeit werden Handlungsmuster von Lehrpersonen im wirtschaftsberuflichen Unterricht betrachtet.

3 Die Kategorie Berater (Mutter/Vater, Freund, Psychologe, Gefährte) wurde von Saban, Kocbeker \& Saban (2007) formuliert und inhaltsanalytisch von den Autoren abgesichert. 
Abbildung 1: Variablenmodell (Basisversion) mit Beispielen zur Illustration der vermuteten Beziehung

Variable 1

Metaphern

zur Umschreibung der

Lehrertätigkeit

Konzeptuelle Kategorie nach

Saban, Kocbeker \& Saban (2007)

Berater

Ein Lehrer ist wie...

...ein Freund, der ein offenes Ohr für die Probleme der Schüler hat.

oder

...eine Mutter oder ein Vater seiner Schüler, weil beide versuchen, ihre Kinder auf das Leben vorzubereiten.
Variable 2

Planung und Realisierung von Lernumgebungen

(hier: Handlungsmuster von Lehrpersonen im wirtschaftsberuflichen Unterricht)

stabil

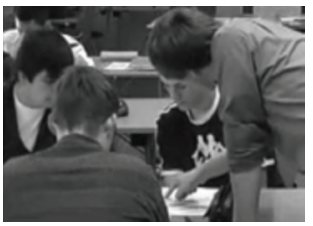

substanziell

Berufsfachschulklasse

Thema: Ver- bzw. Überschuldung junger Konsumenten

Gruppenarbeit zur Erstellung eines Haushaltsplans

Falls eine stabile, substanzielle sowie gleichgerichtete Beziehung zwischen diesen beiden Variablen existiert, könnte die Einschätzungen dieser Metaphern (1) vor und / oder (2) im Rahmen der Lehreraus- und -weiterbildung erhoben werden. In dem zuerst genannten Anwendungsfeld wäre dann zu prüfen, ob sie als Kriterium zur effizienten Feststellung der Eignung für ein „Lehramtsstudium“ (z. B. den Masterstudiengang „Wirtschaftspädagogik“) genutzt werden sollen ${ }^{4}$ oder (erst) im zweiten Anwendungsfeld zur adressatengerechteren und somit effektiveren Gestaltung des Programms (bzw. der enthaltenen Lehrveranstaltungen) eingesetzt werden. Beispielsweise könnten die Veranstaltungsteilnehmer/-innen gezielt mit den eigenen bzw. von anderen für treffend befundenen Metaphern konfrontiert und daran anknüpfend bei Bedarf - gezielt reflexionsbasierte Veränderungen eingeleitet werden. Diese und weitere Überlegungen zum Einsatz von Metaphern sowie Anwendungsmöglichkeiten im Rahmen der Lehrerbildung diskutieren auch Mahlios, Massengill-Shaw \& Barry (2010).

Im Rahmen des Beitrags werden zunächst der theoretische Hintergrund und der Stand der Forschung skizziert. Danach wird über die Fragestellung

4 Mit dem Instrument soll lediglich das Potential zur Entwicklung im Rahmen der Lehrerbildung und nicht die ,endgültige' Eignung getestet werden: Die Wahl bestimmter Metaphern indizieren bestimmte Kognitionen, die sich entwicklungsverhindernd, -hemmend oder fördernd auswirken könnten. 
und die Hypothesen informiert, das Untersuchungsdesign beschrieben und es werden Ergebnisse der Studie 4 präsentiert. Abschließend wird die wirtschaftspädagogische Relevanz der Studienreihe aufgezeigt.

\section{Theoretischer Hintergrund}

Um die Untersuchung auf ein solides Fundament zu stellen, gilt es in einem ersten Schritt, den theoretischen Hintergrund der Variable Metapher sowie den Stand der Forschung in dem Feld zu skizzieren. Gemäß der Kognitiven Theorie der Metapher von Lakoff \& Johnson (1980) gelten Metaphern als mächtige mentale Modelle, d. h. reduzierte, aber zugleich elaborierte, analoge kognitive Repräsentationen, die bei Interaktionen mit der Umwelt fortlaufend generiert werden. Nach Guerrero \& Villamil (2002) sind Metaphern (bzw. im übertragenen Sinn deren mentales Äquivalent, das mentale Modell) nicht nur als Produkt vorangegangener, sondern auch als Determinante zukünftiger (Lehr-Lern-)Handlungen aufzufassen. Mentalen Modellen kommt in Bezug auf das Wahrnehmen, Denken und Handeln eine orientierende Funktion $\mathrm{zu}^{5}$. Sie bauen auf Analogiebeziehungen auf, die anhand von Metaphern umschrieben werden können. Metaphern stellen somit ein Mittel zur Veranschaulichung von Analogien dar (Dutke, 1994).

Metaphern können als effektives sowie effizientes Werkzeug fungieren, da mit ihnen Informationen verdichtet („Brennglasfunktion“) sowie bestimmte Merkmale hervorgehoben („Highlightfunktion“) werden. Darüber hinaus wird durch das Erzeugen authentischer Assoziationen, ein leichter Zugang zu mental ablaufenden Prozessen ermöglicht, was sich für das Erklären neuer Sachverhalte („Elaborationsfunktion“), Diskurse (,Kommunikationsfunktion“) und daher zum Anstoßen von Cognitive Change Prozessen („Reflexionsfunktion") besonders gut eignet. Guski (2007) hat die Funktionen metaphorischer Konzepte im Bereich Pädagogik, inkl. deren Vorzüge und Grenzen umfassend zusammengestellt und diskutiert, wobei die Systematik und Prägnanz der Darstellung noch verbessert werden könnte.

5 Im Rahmen weiterer Ausarbeitungen sollte auch der Bezug zu dem Konstrukt „Sichtweisen von Lehrpersonen“ bzw. assoziierter Ansätze wie „Überzeugungen“ oder „Subjektive Theorien" hergestellt werden (zusammenfassend siehe Seifried, 2010). 


\section{Stand der Forschung ${ }^{6}$}

In einigen Studien wurde untersucht, (1) wie direkt bzw. indirekt am Unterricht Beteiligte mit Hilfe von Metaphern die Lehrertätigkeit umschreiben, und (2) Unterschiede bzw. vereinzelt Veränderungen in Bezug auf deren Metaphernwahl analysiert: Beispielsweise hinsichtlich Metaphern,

- die von Lehrpersonen und Schüler/-innen (Inbar, 1996),

- die von Lehrpersonen in leistungsstarken bzw. leistungsschwachen Klassen (Ben-Peretz, Mendelson \& Kron, 2003),

- die von erfahrenen Lehrpersonen und Lehramtsstudierenden (Martinez, Sauleda \& Huber, 2001; Alger, 2009; Seferoğlu, Korkmazgil \& Ölçü, 2009),

- die von Lehramtsstudierenden mit unterschiedlichem Geschlecht, Studiengang und Studienabschnitt (Saban, Kocbeker \& Saban, 2007)

verwendet bzw. für treffend befunden werden.

Im Rahmen mehrerer Studien wurde an der Universität Mannheim begonnen zu eruieren, ob diese Befunde auch für die Wirtschaftspädagogik gültig sind und prognostisch genutzt werden können ${ }^{7}$ : Mit Studie 1 wurde zunächst sondiert, ob sich die Befunde von Saban, Kocbeker \& Saban (2007) replizieren lassen, und es somit lohnenswert erscheint, das Forschungsvorhaben weiter zu verfolgen. Hierzu wurden die 63 von Saban, Kocbeker \& Saban (2007) dokumentierten Metaphern übersetzt und im Folgenden von den Probanden ( $\mathrm{N}=407$, rund $60 \%$ der Immatrikulierten) auf einer fünfstufigen Likert-Skala von $1=$,gar nicht treffend“ bis $5=$,,sehr treffend“ eingeschätzt.

Um die gemäß den Ergebnissen der Studie 1 bestehenden soziokulturellen Unterschiede, aber auch Gemeinsamkeiten näher zu erkunden, wurde die Studie 2 konzipiert. Die Skala wurde dahingehend modifiziert, dass die Befragten ( $\mathrm{N}=107$, knapp 15\% der Immatrikulierten) zunächst via Unter-

6 Aufgrund des begrenzten Platzkontingents können nur einige ausgewählte Studien thematisch geordnet genannt werden. Eine zusammenfassende Beschreibung der Zielsetzung und Ergebnisse der Studien sowie kritische Anmerkungen zur Aussagekraft einiger Studien finden sich in Lehmann \& Ebner (2011) und Ebner \& Lehmann (2012). In den beiden Artikeln wird auch zu den verschiedenen inhaltlichen Kategorisierungsmöglichkeiten von Metaphern Bezug genommen, die im Rahmen der Studien extrahiert werden.

7 Bei der Studie 1 und 2 handelt es sich um Pilotstudien mit Wirtschaftspädagogen der Universität Mannheim. Aus Effizienzgründen werden erst unter (4.) deren zentrale Ergebnisse mit den Ergebnissen der Studie 4 zusammenfassend dargestellt. Studie 3 thematisiert die Einschätzung von Studierenden der Wirtschaftspädagogik hinsichtlich der Rolle des Schülers und soll lediglich verwendet werden, um die Komplementarität der Einschätzungen zu kontrollieren. Sie findet im Rahmen dieser Ausarbeitung keine weitere Berücksichtigung. 
streichen eine Vorselektion der 63 Metaphernanhänge (z.B. „Ein Lehrer ist wie eine Quelle, ...") vornehmen und danach die zehn am treffendsten befundenen vervollständigen sollten. Darüber hinaus wurde im Anschluss die Möglichkeit eröffnet, selbst zusätzliche Metaphern zu kreieren.

Die Verdichtung der Metaphern zu statistisch nachweisbar reliablen Bündeln ist bislang nur ansatzweise gelungen und soll daher im Rahmen der Studie 4 fortgesetzt werden. Diese Aufgabe stellt eine besondere Herausforderung dar, da bei komplexen Phänomenen (wie der Tätigkeit von Lehrpersonen) Menschen häufig mehrere Metaphern benutzen, um ihren Vorstellungen einen aus ihrer Sicht angemessenen Ausdruck zu verleihen.

\section{Fragestellung und Hypothesen ${ }^{8}$}

Unter Berücksichtigung der quantitativen und qualitativen Auswertung der Studie 2 sowie einer Gegenüberstellung der Ergebnisse aus den vorherigen (Pilot-)Studien soll in der Studienreihe ein (stabiles) Set an Metaphern zur reliablen Umschreibung der Tätigkeit von Lehrpersonen entwickelt werden. Mit Hilfe des Instruments soll am Ende der Studienreihe zuverlässig das Vorliegen von mentalen Modellen hinsichtlich der (prospektiven) Berufstätigkeit erhoben und effizient - d.h. ohne den Einsatz weiterer zeit- und kostenaufwendiger Erhebungsinstrumente - Handlungsmuster im (wirtschaftsberuflichen) Unterricht valide prognostiziert werden können.

In Studie 4 gilt es neben der Replikation der Befunde der Pilotstudien in einer weitergehenden Prüfung, die Bedeutung der Metaphernbestandteile (insbesondere der Begründungen) zu analysieren. Des Weiteren werden bereits getestete sowie weitere personengruppenbezogene Unterschiede untersucht, um zu eruieren, ob sich die Ergebnisse früherer Studien und weitere bei (angehenden) Lehrpersonen (beruflicher) Schulen in unserem Land bzw. Berufsfeld zeigen.

\section{Untersuchungsdesign}

Es handelt sich bei der hier vorrangig betrachteten Studie 4 um eine Querschnittstudie mit deskriptivem Untersuchungsdesign, die schrittweise zu ei-

8 Die zu prüfenden Hypothesen entsprechen jenen, die in den Pilotstudien unter Berücksichtigung der Befunde von Saban, Kocbeker \& Saban (2007) formuliert wurden. 
ner Längsschnittstudie ${ }^{9}$ ausgebaut werden soll. Nach Möglichkeit sollen in Folgestudien ebenfalls (quasi-)experimentelle Designs (Versuchs- und Kontrollgruppen) angewendet werden, um die Effekte der sich in Metaphern materialisierenden mentalen Modellen auf die Handlung(-smuster) von Lehrpersonen im wirtschaftsberuflichen Unterricht messen zu können.

\section{Probanden}

Befragt wurden insgesamt 296 Studierende der Wirtschaftspädagogik an der Universität Mannheim, die sich freiwillig gemeldet haben und für das Ausfüllen des Fragebogens eine VPn-Stunde erhalten. Neun Datensätze konnten aufgrund von Inkonsistenzen nicht berücksichtigt werden. Der bereinigte Datensatz umfasst somit die Antworten von 287 Befragten (über ein Drittel der Immatrikulierten). Davon waren 232 im Bachelor-, 28 im Master- und 26 im Diplomstudiengang immatrikuliert; eine Person lieferte keine Angabe zum Studiengang. 65,5 Prozent der Probanden sind weiblich; als Berufswunsch geben 40,8 Prozent sehr wahrscheinlich Lehrer/-in an, 25,4 bzw. 10,7 Prozent möchten sehr wahrscheinlich eine kaufmännische Tätigkeit im Bereich Personal bzw. einem anderen Bereich ausüben, 5,8 Prozent streben eine andere Tätigkeit an. 46,2 Prozent der Befragten befinden sich im ersten bzw. zweiten Fachsemester des Bachelorstudiengangs, 35,0 Prozent im dritten oder höheren Fachsemester des Bachelorstudiengangs und 18,9 Prozent im Masterbzw. Diplomstudiengang.

\section{Erhebungsinstrument}

Die Modifikationen des Fragebogens betreffen eine Best-of-Auswahl aus den Metaphern der Pilotstudien sowie sprachliche Überarbeitungen. Darüber hinaus wurden weitere Metaphern ergänzt, die in der pädagogischen Fachliteratur häufig genannt oder in anderen Studien getestet wurden. Der Fragebogen besteht aus 47 Items, davon wurden 36 aus Studie 1 übernommen bzw. auf Basis der Ergebnisse von Studie 2 modifiziert und elf neue Metaphern auf Basis der Ergebnisse von Studie 2 bzw. anderer Literaturquellen integriert $(\text { Gesamtskala } \alpha=.93)^{10}$.

9 Um ein Matching der Probanden in nachfolgenden Studien zu ermöglichen, wurde ein Codesystem in den Fragebogen integriert.

10 Die Skala wurde als Teil B in ein umfassenderes Erhebungsinstrument integriert. 
Es wurde eine sechsstufige Likert-Skala mit zwei Ankerpunkten eingesetzt (1 $=$, gar nicht treffend" bis $6=$, ,sehr treffend" $)^{11}$.

Des Weiteren wurden bei der Zusammenstellung des Erhebungsinstruments Fragen zu (demographischen) Merkmalen der Probanden ${ }^{12}$ [vor Teil A] und weitere Skalen zur Erfassung ausgewählter individueller Merkmale inkludiert [z.B. Teil D: Lehrerbezogene Selbstwirksamkeitserwartung ${ }^{13}$ ].

\section{Datenerhebung und-auswertung}

Die Befragung wurde an vier Erhebungsterminen in den Räumlichkeiten der Universität Mannheim durchgeführt. Die Probanden wurden zur Sicherstellung der Durchführungsobjektivität von einem wissenschaftlichen Mitarbeiter des Lehrstuhls für Wirtschaftspädagogik einheitlich instruiert. $\mathrm{Zu}$ jeder Erhebung wurde ein Protokoll erstellt. Die Dauer der Erhebung betrug 52-60 Minuten; die berechnete durchschnittliche Bearbeitungsdauer 36,26 Minuten.

Es wurden deskriptive Analysen, Mittelwertvergleiche und Faktorenanalysen durchgeführt. Hinsichtlich der Kontrollvariable Bearbeitungsdauer [T-Test für unabhängige Stichproben mit dem Trennwert 36,26] ergeben sich signifikante Unterschiede $(\mathrm{p} \leq .05)$ bei den Metaphern Dirigent 1 und Jongleur sowie Krug und Oberleutnant - die Probanden, die sich länger Zeit als der Durchschnitt genommen haben, werten die beiden erst genannten Metaphern als treffender und die beiden zuletzt aufgeführten als weniger treffend. Insgesamt zeigt sich, dass diejenigen, die zumindest tendenziell eher eine Lehrertätigkeit ergreifen möchten, eine längere Bearbeitungszeit aufweisen.

11 Die Änderung der Ausprägungsstufen von einer fünf- auf eine sechsstufige Likert-Skala war aus forschungsmethodischen Gründen erforderlich, damit zumindest eine Tendenzentscheidung von den Befragten vorgenommen wird. Bei der späteren Betrachtung der Ergebnisdarstellung ist dieser Aspekt zu beachten. Zur Sicherstellung der Vergleichbarkeit der Ergebnisse wird in zukünftigen Untersuchungen mit einer sechsstufigen Likert-Skala gearbeitet.

12 Unter anderem Geschlecht, Studiengang, Fachsemester und vier Arten der angestrebten beruflichen Tätigkeiten mit Einschätzung auf einer vierstufigen Likert-Skala von $1=$,sehr unwahrscheinlich“ bis 4 = ,sehr wahrscheinlich“".

13 Adaptierte OSTES-Skala von Tschannen-Moran \& Woolfolk Hoy (2001) mit einer neunstufigen Likert-Skala $(1=$,überhaupt nicht“ bis $9=$,sehr viel/gut" $)$, die von 98 Probanden hinsichtlich des Berufseinstiegsjahres (BEJ) $(\alpha=.89)$ und von 189 hinsichtlich des Wirtschaftsgymnasiums (WG) $(\alpha=.92)$ als Tätigkeitsfeld eingeschätzt wurden. 


\section{Ergebnisse}

In Studie 4 konnten wesentliche Befunde der Pilotstudien repliziert und das Metaphernset stabilisiert werden. In Bezug auf die Studie 4 ergeben sich folgende Ergebnisse und Folgerungen [Erläuterung nach dem Pfeil] aus der deskriptiven Analyse (siehe Abbildung 2):

Abbildung 2: Deskriptive Analyse der Studie 4 (inkl. Bezugnahme auf die Ergebnisse der Studien 1 und 2)

Studie $4^{6 \text {-stufige Likert-Skala (Verlaufsrichtung: Je höher, desto treffender.) }}$

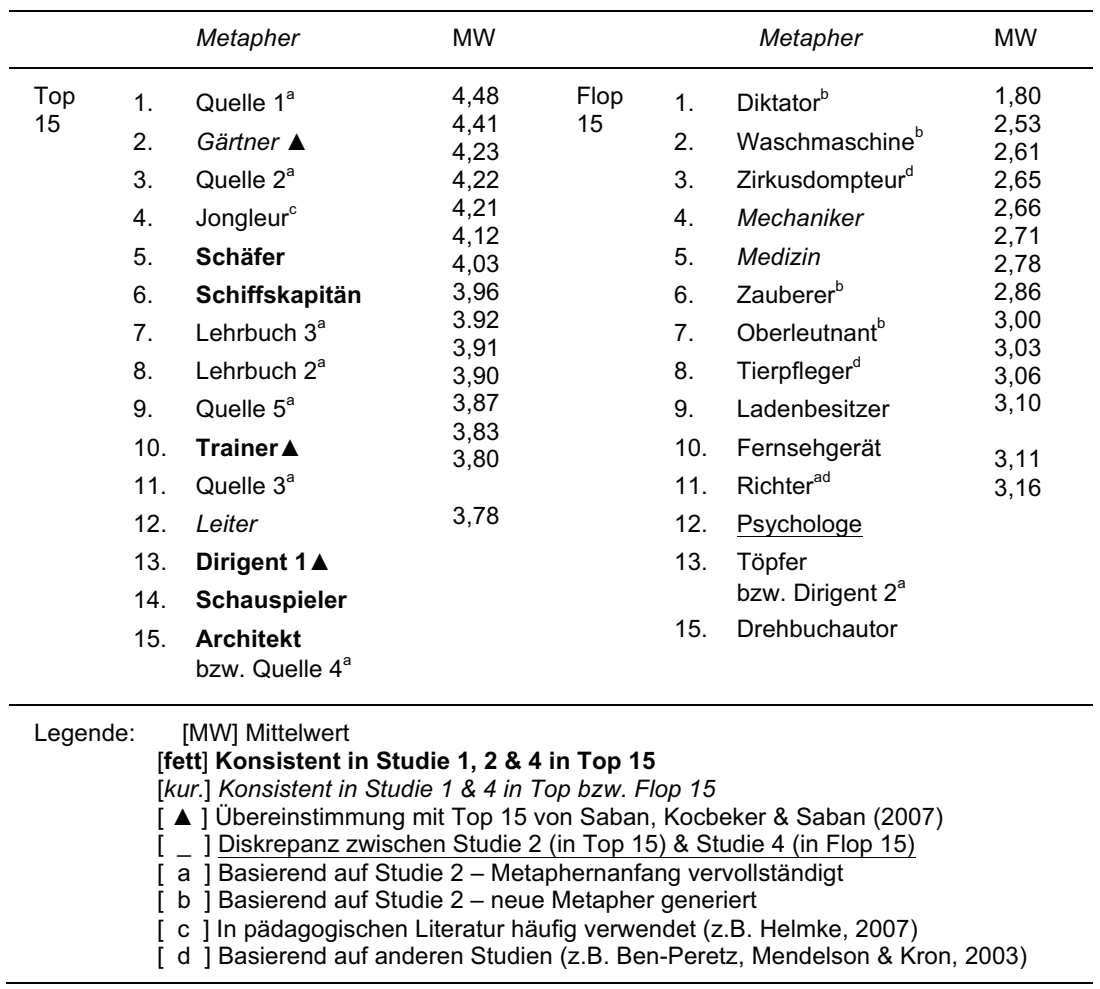

- Quelle 1, Gärtner, Quelle 2, Jongleur und Schäfer werden am höchsten (am treffendsten); Diktator, Waschmaschine, Zirkusdompteur, Mechaniker und Medizin am niedrigsten (am wenigsten treffend) gewertet. 
- Gärtner und Leiter sind unter den Top 15 in der Studie 1, jedoch nicht in Studie 2. Schäfer, Schiffskapitän, Trainer, Dirigent 1, Schauspieler und Architekt sind wie in Studie 1, 2 und 4 in den Top 15 enthalten. Die Hypothese 4 in Lehmann \& Ebner (2011) wird somit weiter untermauert.

- Gärtner, Trainer und Dirigent 1 sind ebenfalls in den Top 15 der bei Saban, Kocbeker \& Saban (2007) am häufigsten genannten Metaphern aufgeführt, was die Hypothese 1 in Lehmann \& Ebner (2011) stützt ${ }^{14}$.

- Alle fünf Ausdifferenzierungen der in Studie 2 zweitplatzierten Metapher Quelle und zwei der drei Ausdifferenzierungen der in Studie 2 an der Spitze platzierten Metapher (Lehr-)Buch sind unter den Top 15.

- Abweichend von Studie 2 ist die damals am vierthäufigsten zur Vervollständigung ausgewählte Metapher Psychologe in den Flop 15 gelistet. Darüber hinaus sind abweichend von Studie $1 \& 2$ sowie Saban, Kocbeker \& Saban (2007) sind Kompass und Mutter/Vater nicht in den Top 15 zu finden.

$\rightarrow$ Eine (Rück-)Modifikation der Metaphern Psychologe und Kompass sowie eine Prüfung der Zweckmäßigkeit der Ausdifferenzierung von Mutter/Vater ist folglich in Betracht zu ziehen.

Neben den ,Signalwörtern' der Metapher spielt die jeweilige Begründung eine entscheidende Rolle. Diese Sensitivität lässt sich gut anhand der ausdifferenzierten Metaphern Quelle und Dirigent nachweisen. Die auf harmonische Zusammenarbeit orientierte Metapher Dirigent 1 taucht unter den Top 15 auf, die eine Leitungsfunktion betonende Metapher Dirigent 2 (Takt vorgeben) hingegen in den Flop 15. Die den Aspekt der Hilfestellung hervorhebende Metapher Quelle 3 ist wie die Metaphern Quelle 1, 2, 4 und 5, die verschiedene Facetten der Wissensarbeit umschreiben, in den Top 15 aufgeführt. In den Faktorenanalysen zeigt sich jedoch die andere inhaltliche Ausrichtung: Bei der Einstellung Eigenwert $>1$ werden die Metaphern der Studie 4 in zehn Faktoren (Varianzaufklärung 58,94 Prozent) geordnet, die sich allerdings nicht vollständig inhaltlich sinnvoll bezeichnen lassen. Unter inhaltlichen Überlegungen auf Basis der mit Varimax rotierten Komponentenmatrix der 10-Faktoren-Lösung sowie des Screeplots wurde daher die Berechnung einer 6-Faktoren-Lösung (Varianzaufklärung 48,72 Prozent) durchgeführt. Eine durchgängige Stabilität weisen jeweils die ersten drei Faktoren auf: (1) Wis-

14 Korrigierend ist darauf hinzuweisen, dass die Metapher Schiffskapitän nicht - wie in Lehmann \& Ebner (2011) und Ebner \& Lehmann (2012) beschrieben - in den Top Ten von Saban, Kocbeker \& Saban enthalten ist; allerdings wird ihr bei den Studien 1, 2 und 4 eine bedeutsame Rolle zuteil. 
sensarbeit(er) $)^{15}$, (2) Bezugsperson / soziale Unterstützung ${ }^{16}$ und (3) determinierte \& determinierende Tätigkeit ${ }^{17}$ (die drei Faktoren liefern eine Varianzaufklärung von 37,18 Prozent). Die Stabilität der Landungen der 24 Metaphern der drei Faktoren wurde in einer weiteren Faktorenanalyse geprüft (Varianzaufklärung 53,18\%; nur Metapher Medizin liegt knapp unter der Ladungsgrenze von .5). Bezugnehmend auf die Hypothese 3 in Lehmann \& Ebner (2011) können im Rahmen der Studie 4 die konzeptuellen Kategorien ebenfalls nicht übereinstimmend anhand statistischer Auswertungen nachgewiesen werden. Allerdings zeigen sich inhaltsbezogene Affinitäten (z.B. hinsichtlich der Kategorie Berater).

Zwischen einzelnen Probandengruppen wurden auf der Metaphernebene folgende substanzielle Unterschiede ermittelt (Signifikanzniveau $\mathrm{p} \leq .05)^{18}$ :

- Geschlecht: [T-Test für unabhängige Stichproben]

Frauen > Männer bei Quelle 1, Leiter \& Quelle 3.

- Berufswunsch: [T-Test für unabhängige Stichproben mit Trennwert 3 auf einer vierstufigen Likert-Skala $\left.{ }^{19}\right]$

Lehrtätigkeit

$[3,4]>[1,2]$ bei Freund, Arzt, Quelle 3, Mutter/Vater 3 \& 4;

$[1,2]>[3,4]$ bei Waschmaschine, Oberleutnant \& Diktator. Andere kaufmännische Tätigkeit

$[3,4]>[1,2]$ bei Diktator;

$[1,2]>[3,4]$ bei Freund, Arzt, Gefährte, Quelle 3, Mutter/Vater 3 und Psychologe.

- Studiengang / Fachsemester (FS) [ANOVA mit den vier Gruppen Bachelor 1.-2. FS, Bachelor 3.+ FS, Master, Diplom]

Bachelor 1.-2. FS > Master bei Quelle 2, Test, Lehrbuch 3 und Dirigent 2;

Bachelor 3.+ FS > Master bei Waschmaschine.

15 Für (1) Wissensarbeit(er) weisen folgende sieben Metaphern Ladungen $>.5$ auf $(\alpha=.90)$ : Quelle 1, 2, 4, 5 und Lehrbuch 1 - 3. Kennzeichnend ist die Rolle der Lehrperson als Besitzer und Distributor von Wissen bzw. des Schülers als Wissensnutzer.

16 Für (2) Bezugsperson / soziale Unterstützung weisen folgende sieben Metaphern Ladungen $>.5$ auf $(\alpha=.87)$ : Mutter/Vater $1-4$, Freund, Gefährte und Quelle 3. Kennzeichnend sind die Hilfestellung / Rat gebende Rolle der Lehrperson.

17 Für (3) determinierte \& determinierende Tätigkeit weisen folgende zehn Metaphern Ladungen $>.5$ auf $(\alpha=.84)$ : Zirkusdompteur, Drehbuchautor, Richter, Waschmaschine, Test, Mechaniker, Oberleutnant, Dirigent 2, Medizin und Diktator. Kennzeichnend ist die aktive Rolle der Lehrperson (und die eher rezeptive Sicht auf den Schüler / die Schülerin).

18 „> " bedeutet die Metaphern der zuerst genannten Gruppe werden im Vergleich zu der anderen als treffender eingestuft.

$191=$,sehr unwahrscheinlich“ bis 4 =,,sehr wahrscheinlich“; [3,4] wird im Folgenden als mindestens eher wahrscheinlich und $[1,2]$ mindestens eher unwahrscheinlich interpretiert. 
- Lehrerbezogene Selbstwirksamkeitserwartung [T-Test für unabhängige Stichproben mit Mediansplit als Trennwert: BEJ 6,58 und WG 6,75] BEJ

Höhere > niedrigere bei fast zwei Drittel (31) der Metaphern.

Exklusive Unterschiede bei Architekt, Trainer, Quelle 2, Arzt, Schmied, Quelle 3, Baumeister, Mechaniker, Psychologe, Dirigent 2 und Töpfer.

WG

Höhere > niedrigere bei über die Hälfte (22) der Metaphern.

Exklusive Unterschiede bei Gärtner, Zirkusdompteur, Lehrbuch 1 \& 2 und Jongleur.

Die in Lehmann \& Ebner (2011) formulierte Hypothese 2, dass sich ähnliche personengruppenbezogene Unterschiede wie in der Bezugsstudie zeigen, wird hiermit partiell weiter untermauert. Weitere Unterschiede sind feststellbar.

\section{Wirtschaftspädagogische Relevanz}

Im Rahmen der Studie 4 konnten alle in Lehmann \& Ebner (2011) formulierten Hypothesen (zumindest partiell) weiter gestützt werden. Es wurden einige reliable Metaphern zur Umschreibung der Lehrertätigkeit identifiziert und ein weiterer Schritt in die Richtung eines validen Sets an Metaphern ist unternommen. Gruppenbezogene Unterschiede wurden ebenfalls ermittelt, auf die in weiteren Studien zu achten sein wird (insbesondere bei experimentellem Untersuchungsdesign zur Kontrolle der adäquaten Zusammensetzung der Vergleichsgruppen). Die faktorenanalytisch stabilen bzw. in mindestens zwei der drei Studien in der Top 15 rangierenden Metaphern sollen in der folgenden Studie 5 getestet werden, um das Set hinsichtlich der Metaphernzusammensetzung möglichst ökonomisch und gleichfalls valide zu gestalten. $\mathrm{Zu}$ prüfen ist in diesem Zusammenhang insbesondere, ob sich verbliebene starke „Solo-Metaphern“ zu einer weiteren inhaltlichen Facette bündeln lassen.

Mit dem stabilen Metaphernset soll am Ende der Studienreihe untersucht werden, ob sich die ermittelte(n) Vorstellung(-smuster) im unterrichtsbezogenen Handeln (Handlungsmuster) von Lehrpersonen manifestieren. Falls dies zutrifft, könnte es vor und / oder im Rahmen der Lehrerbildung eingesetzt werden: Das Instrument könnte zum einen effizient zur Auswahl und zum anderen zur Professionalisierung von Wirtschaftspädagogen / (angehenden) Lehrpersonen beitragen, z.B. bei Kommunikations- / Reflexionsprozessen.

Zwischen den Gruppen mit unterschiedlichen Berufswünschen sowie verschiedener Studienphasen liegen signifikante Unterschiede vor. Aufgrund der 
Offenheit der wirtschaftspädagogischen Studiengänge im Hinblick auf die berufliche Perspektive erscheint die Entwicklung des Instruments folglich besonders interessant. In den Folgestudien der Studienreihe sollten die theoretischen Hintergründe dieses Phänomens näher untersucht werden.

\section{Literatur}

Alger, C. A. (2009). Secondary teachers' conceptual metaphors of teaching and learning: Changes over the career span. Teaching and Teacher Education, 25, pp. 743-751.

Ben-Peretz, M., Mendelson, N. \& Kron, F.W. (2003). How teachers in different educational contexts view their roles. Teaching and Teacher Education, 19, pp. $277-$ 290.

Dutke, S. (1994). Mentale Modelle: Konstrukte des Wissens und Verstehens. Göttingen, Stuttgart: Verlag für Angewandte Psychologie.

Ebner, H. G. (2010). Konzeptuelle Grundlagen des Managements beruflicher Schulen. In R. Nickolaus, G. Pätzold, H. Reinisch \& T. Tramm (Hrsg.), Handbuch Berufsund Wirtschaftspädagogik (S. 267-280). Bad Heilbrunn: Klinkhardt.

Ebner, H. G. \& Lehmann, B. (2012). Mit Metaphern den Facetten der Tätigkeit von Lehrpersonen auf der Spur. Die berufsbildende Schule. (in Druck)

Fend, H. (2002). Mikro- und Makrofaktoren eines Angebot-Nutzungsmodells von Schulleistungen. Zeitschrift für Pädagogische Psychologie, 16(3/4), S. 141-149.

Guerrero, M. C. M. \& Villamil, O. S. (2002). Metaphorical conceptualizations of ESL teaching and learning. Language Teaching Research, 6(2), 95-120.

Guski, A. (2007). Metaphern der Pädagogik. Metaphorische Konzepte von Schule, schulischem Lernen und Lehren in pädagogischen Texten von Comenius bis zur Gegenwart. Bern u.a.: Lang.

Helmke, A. (2007). Unterrichtsqualität. Erfassen - Bewerten - Verbessern (5. Auflage). Seelze: Kallmeyer.

Inbar, D. (1996). The free educational prison: metaphors and images. Educational Research, 38(1), S. 77-92.

Lakoff, G. \& Johnson, M. (1980). Metaphors we live by. Chicago, London: The University of Chicago Press.

Lehmann, B. \& Ebner, H. G. (2011). „Ein Lehrer ist wie...“: Mit welchen Metaphern umschreiben Studierende der Wirtschaftspädagogik die Tätigkeit von Lehrpersonen?. In U. Faßhauer, J. Aff, B. Fürstenau \& E. Wuttke (Hrsg.), LehrLernforschung und Professionalisierung: Perspektiven der Berufsbildungsforschung. Schriftenreihe der Sektion Berufs- und Wirtschaftspädagogik der Deutschen Gesellschaft für Erziehungswissenschaft (DGfE) (S. 135-145). Opladen \& Farmington Hills: Budrich.

Mahlios, M., Massengill-Shaw, D. \& Barry, A. (2010). Making sense of teaching through metaphors: a review across three studies. Teachers and Teaching: theory and practice, 16(1), pp. 49-71. 
Martínez, M. A., Sauleda, N. \& Huber, G. L. (2001). Metaphors as blueprints of thinking about teaching and learning. Teaching and Teacher Education, 17, pp. 965-977.

Saban, A., Kocbeker, B. N. \& Saban, A. (2007). Prospective teachers' conceptions of teaching and learning revealed through metaphor analysis. Learning and Instruction, 17, pp. 123-139.

Seferoğlu, G., Korkmazgil, S. \& Ölçü, Z. (2009). Gaining insights into teachers' ways of thinking via metaphors. Educational Studies, 35, pp. 323-335.

Seifried, J. (2010). Sichtweisen von Lehrpersonen an kaufmännischen Schulen. Zeitschrift für Berufs- und Wirtschaftspädagogik, 106(2), S. 199-219.

Tschannen-Moran, M. \& Woolfolk Hoy, A. (2001). Teacher efficacy: capturing an elusive construct. Teaching and Teacher Education, 17, pp. 783-805. 



\section{Erfolgreich Studieren - Entwicklung und Validierung eines Fragebogens zur Erfassung studienbezogener Selbstregulationsprozesse}

Sandra Trost

\section{Problemstellung und Motivation}

Folge der vielzitierten Megatrends (Buttler, 1992) ist eine Bevölkerung, die zunehmend heterogener, individualisierter und hochqualifizierter ist (u. a. Baethge, Achtenhagen, Arends, Babic, Baethge-Kinsky \& Weber, 2006; Picot, Reichwald \& Wigand, 2001). Damit einher geht ein entscheidender Wandel in der Arbeitswelt und im privaten Leben der Individuen. Zentrale Veränderungen in der Lernumwelt Universität begründen sich in den Reformen des Bologna-Beschlusses von 1999. Das zweistufige (Bachelor/ Master), modularisierte, an Leistungspunkten orientierte und auf internationale Wettbewerbsfähigkeit hin ausgerichtete System stellt die Studierenden vor neue Herausforderungen (Hochschulrektorenkonferenz, 2004, S. 31). Beobachtet werden zunehmender Leistungsdruck, Unsicherheiten bezüglich eines optimalen Studienfortgangs und der Akzeptanz am Arbeitsmarkt sowie kaum reduzierte Stoffpläne in den Bachelormodulen gegenüber den Curricula der Diplomstudiengänge. Studierende klagen über zunehmende Belastungen und Stresssituationen bis hin zum Burnout (u.a. Gusy, Lohmann \& Drewes, 2010). Diese Tendenz spiegelt sich zum einen in den nach wie vor erschreckend hohen Drop-out Quoten wieder: Fast jeder dritte Bachelorstudent, der sich im Zeitraum 2000-2004 immatrikulierte, brach sein Studium ab (Heublein, Hutzsch, Schreiber, Sommer \& Besuch, 2010, S. 9-10). Zum anderen resignieren Studenten in Bachelor-Studiengängen deutlich früher - im Schnitt nach 2,3 Semestern - als Studierende anderer Abschlussarten (Heublein et al., 2010, S. 48). Erfolgreich studieren in einer sich ändernden Lebensund Lernumwelt bedeutet mehr als nur die Anwendung aufgaben- bzw. problemorientierter Lernstrategien; es geht vielmehr um die Fähigkeit, übergreifende Lebensentwürfe gestalten und Herausforderungen bewältigen zu können. Dazu gehört die aktive Gestaltung der eigenen Karriere, das zielgerichtete, autonome und selbstgesteuerte Vorantreiben von Studien- und Lebensprojekten (Familie, Freunde, Studienfinanzierung, Vereinsarbeit, etc.) sowie die erfolgreiche Bewältigung von Belastungen und Misserfolgen.

Die traditionelle Vermittlung an Universitäten, die sich zumeist auf fachwissenschaftliche Inhalte beschränkte und auf kurzfristige und individuelle 
Lernprozesse hin ausgerichtet war (Weber, 2005, S. 12), ist nicht länger ausreichend. „Die Anforderungen des modernen Lebens“ (OECD, 2008, S. 8) verlangen zusätzlich nach „Schlüsselkompetenzen“, um sich im jeweiligen gesellschaftlichen Umfeld angemessen bewegen und behaupten zu können. Das DeSeCo-Projekt (Definition and Selection of Key Competencies) der Organization for Economic Co-Operation and Development (OECD) weist drei zentrale zukünftige Kompetenzen aus: (I) die interaktive Anwendung von Medien und Mitteln; (II) das Interagieren in heterogenen Gruppen und (III) das eigenständige (autonome) Handeln (OECD, 2008). Die vorliegende Studie beschäftigt sich mit der Schlüsselqualifikation ,eigenständiges Handeln“ im Kontext studienbezogener Selbstregulation. Dabei geht es um das Phänomen der selbstständigen Setzung von Zielen im Studium sowie der erfolgreichen Verfolgung und Erreichung von Studienzielen im Wettstreit konkurrierender Lebensprojekte. Auf der Grundlage von Wissen und Informationen erfolgen Wahrnehmungs-, Abwägungs-, Selbsteinschätzungs- und Entscheidungsprozesse sowie die Interaktion mit der physischen und sozialen Umwelt. Konkret bedeutet dies, das Lebensprojekt „erfolgreich Studieren“ zu planen, zu managen bzw. zu bewältigen.

Zur Operationalisierung studienbezogener Selbstregulation wird auf den Ansatz der "Selektion, Optimierung und Kompensation“" von Baltes und Kollegen (bspw. Baltes \& Baltes, 1989; Freund \& Baltes, 2000, 2002) aus der Lebensspannenpsychologie zurückgegriffen. Forschungen in diesem Bereich ergaben, dass eine plurale Selbststruktur die Adaptation an veränderte Entwicklungsbedingungen im Zuge selbstregulativer Prozesse erleichtert (Lindenberger, 2006, S. 387) oder dass eine Person durch den Besitz von verschiedenartigen Bewältigungsstrategien ihre Widerstandskraft gegen Stressoren scheinbar stärken kann (Staudinger, Marsiske \& Baltes, 1995).

Zur Gestaltung von Trainings bzw. zur Identifikation von Hinweisen für die Studienberatung zur erfolgreichen Bewältigung des Studiums ist in einem ersten Schritt die Messbarmachung ,studienbezogener Selbstregulationsstrategien" notwendig. Ziel der vorliegenden Studie ist daher die Entwicklung eines reliablen und validen Inventars zur Messung ,studienbezogener Selektions-, Optimierungs- und Kompensationsstrategien“(DSOK).

\section{Die Adaptation des Metamodells der ,Selektion, Optimierung und Kompensation"}

\section{Ursprung und theoretische Fundierung des Modells}

Das SOK-Modell basiert auf drei aus der Lebensspannenpsychologie stammenden Prämissen: (1) Ressourcen sind über die gesamte Lebensspanne 
hinweg beschränkt, (2) Entwicklung ist multidirektional und multifunktional hinsichtlich ihrer adaptiven Anpassungsfähigkeit und (3) Entwicklung ist ein dynamischer Prozess als Reaktion auf die Umwelt sowie die aktive Gestaltung der eigenen Umwelt und der inneren mentalen Prozesse (Freund \& Baltes, 2000, S. 36-37). Entwicklung im Sinne des SOK-Modells ist nach Baltes und seinen Kollegen dann erfolgreich und effizient, wenn die drei Komponenten der Selektion, Optimierung und Kompensation in geeigneter Weise orchestriert werden:

Begründet in der naturgemäßen Ressourcenbegrenzung sind Strategien der (elektiven) Selektion (ES) in Form von Zielauswahl, -setzung, -hierarchisierung und -spezialisierung zentral für die Erklärung menschlichen Handelns und Entwickelns (u.a. Baltes \& Baltes, 1989, S. 96). Durch die Fokussierung auf Ziele in individuell bedeutsamen Handlungsbereichen (z.B. das Managen der Studienorganisation oder das erfolgreiche Bestehen einer ausgewählten Anzahl von Klausuren im aktuellen Semester) erhält Entwicklung zum einen eine wegweisende Richtung und zum anderen kommen individuelle komparative Vorteile besonders zum Tragen (u.a. Freund, 2007, S. 375).

In jüngeren Publikationen der Forschergruppe um Baltes wird eine zweite Komponente zur Beschreibung von Prozessen der Zielsetzung eingeführt: die verlustbasierte Selektion (LBS). Diese Komponente geht von fehlenden zielrelevanten Ressourcen (z.B. fehlende Zeit, Energie) aus. Der verminderte Ressourcenhaushalt (ausgelöst durch Stress oder Misserfolg) ist nicht mehr in der Lage, für die Vielfalt des Zielportfolios (die Anzahl an Klausuren) oder die qualitativ angestrebten Zielzustände (erfolgreich bestehen) die notwendigen Kapazitäten bereitzustellen (Freund, 2007, S. 375). Adaptive Entwicklung geht unter diesen Voraussetzungen mit einer quantitativen oder qualitativen Reduzierung der anvisierten Ziele einher. In einigen Fällen erscheint sogar die Zuwendung zu ganz neuen Zielbereichen als entwicklungsförderliche Entscheidung (Wiese, 2000, S. 69). Der Prozess der Optimierung $(O)$ beschreibt den Erwerb sowie den Einsatz von (z.B. zeitlichen, kognitiven oder motivationalen) Ressourcen, um selbst gesetzte Ziele erfolgreich zu verfolgen und umzusetzen. Handlungstheoretisch formuliert lassen sich folgende Optimierungsstrategien benennen: Investieren von Zeit bzw. anderen Ressourcen, starke bis vollkommene Aufmerksamkeitsfokussierung (z.B. auf das Bestehen der ausgewählten Klausuren), sich ausdauernd für diese Projekte einzusetzen (auch in Lerngruppen oder durch Erfahrungsaustausch) und für seine Vorhaben zu kämpfen. Das Ergreifen eines günstigen Momentes und das Abschauen von Zielverfolgungsstrategien erfolgreicher Anderer ermöglicht einen kreativen Einsatz von Ressourcen (Lindenberger, 2006, S. 355). Kompensation $(K)$ findet ausschließlich in Folge eines erfahrenen Verlustes statt und fokussiert auf die Aufrechterhaltung eines gegebenen Funktionsniveaus (z.B. die Beibehaltung der Anzahl der zu schreibenden Klausuren im aktuellen Semester). Nach Möglichkeit werden alle internen (z.B. noch un- 
genutzte oder aus anderen Lebensbereichen substituierte Energie, Zeit, etc.) sowie externen (z.B. Inanspruchnahme von Beratungsstellen oder anderen Experten wie Coaching oder Mentoring, etc.) Ressourcen noch stärker als bisher mobilisiert (Freund, 2007, S. 377). Im Falle des Studierverhaltens wäre denkbar, dass das eigene Fähigkeitsprofil z.B. um Sprachkenntnisse bzw. Praxiserfahrung ergänzt wird, um ein früher gewohntes, übergreifendes Leistungsniveau (bspw. in Form einer Arbeitsmarktattraktivität) wieder zu erlangen.

\section{Empirische Evidenzen und die Notwendigkeit der domänenspezifischen Adaptation}

Eine Reihe von Untersuchungen aus unterschiedlichen Kontexten und auf Basis verschiedener Altersstufen stützen die Annahme, dass die Anwendung von SOK-Strategien eine adaptive und somit erfolgreiche Entwicklung fördert. Bezogen auf die Lebenswelt junger Erwachsener sei hier auf drei Studien fokussiert: Studienkontext: Wiese \& Schmitz (2002), Karrierekontext: Abele und Wiese (2008), Arbeitskontext: Wiese, Freund und Baltes (2000). Allen Studien gemeinsam sind zum einen die nachgewiesene Anwendbarkeit des SOK-Modells und zum anderen der positive Zusammenhang der SOKStrategieanwendung mit domänenspezifischen Erfolgsvariablen.

Freund und Baltes postulieren, dass ,the 'meta-theory' of SOC needs to be embedded in a specific theoretical framework for applying it to particular developmental phenomena" (Freund \& Baltes, 2000, S. 38). Erfolgt - wie in allen oben genannten Studien der Fall - die Operationalisierung jedoch nur mit einem „Meta“-Fragebogen (Baltes, Baltes, Freund \& Lang, 1999) und soll nur eine kurze Instruktion die Probanden in den jeweiligen Kontext versetzen, leidet sowohl die Messgenauigkeit als auch damit einhergehend die Aussagekraft stark unter den domänenspezifischen Interpretationen der Probanden (Trost, 2011, S. 57-62). Um kontextspezifische „Missverständnisse“ zu vermeiden, wird daher eine domänenspezifische Adaptation des Metamodells vorgeschlagen.

Mittels vier explorativer Vorstudien wurde die Lebenswelt der Studierenden mit ihren typischen Studien- (bzw. Lebens-)projekten sowie den zur Bewältigung eingesetzten domänenspezifischen SOK-Strategien untersucht: (1) eine Befragung von 128 Studierenden zu deren Zielen und Erwartungen, (2) eine Sekundärdatenanalyse des fakultätsinternen Diskussionsforums zur Identifikation typischer kritischer Ereignisse $(\mathrm{N}=30$ Threads, $\mathrm{N}=1030$ Posts), (3) Interviews mit Bachelorstudierenden ( $\mathrm{N}=52)$ bezüglich der Anwendung von SOK-Strategien sowie (4) Interviews mit Experten $(\mathrm{N}=3)$ hinsichtlich kritischer Situationen und deren Lösungsstrategien. 
Aufbauend auf der Literaturanalyse sowie der qualitativen Exploration erfolgte die Entwicklung der Items einer ersten Testversion des adaptierten Fragebogens. Folglich ergibt sich für die vorliegende Studie die Frage nach der Reliabilität und Validität des adaptierten SOK-Instrumentes zur Beschreibung studienbezogener Selbstregulation.

\section{Methode und Durchführung}

\section{Design und Stichprobe}

Im Rahmen der Testentwicklung wurde die erste Testversion zwei weiteren Revisionen und Evaluationen unterzogen: Pretest $(\mathrm{N}=299)$ im März 2008, die längsschnittlich angelegte 1 . Validierungsstudie $(\mathrm{N}=253)$ im April und Juli 2008 sowie die 2. Validierungsstudie $(\mathrm{N}=251)$ im Oktober 2008. Die nachfolgend vorgestellten Ergebnisse beziehen sich auf die im Mai 2009 durchgeführte Hauptstudie zur Replikation der finalen Testversion. Die Datensammlung erfolgte mittels einer inzentivierten Onlinebefragung. Dabei wurde die gesamte Kohorte der Studierenden der Fakultät für Betriebswirtschaft der Ludwig-Maximilians-Universität München auf die Erhebung aufmerksam gemacht. Die Rücklaufquote betrug 10\%. Somit umfasst die Stichprobe N = 297 Bachelor-/ Master- bzw. Diplomstudierende im Alter von durchschnittlich 23,69 Jahren $(\mathrm{SD}=2,78)$ und besteht $\mathrm{zu} 60 \%$ aus Frauen.

\section{Das DSOK-Inventar zur Messung studienbezogener Selbstregulation}

Das im Selbstbericht konstruierte psychometrische Messinstrument besteht aus 7 Items pro Subkomponente: domänenspezifische elektive Selektion (DES), Optimierung (DO), verlustbasierte Selektion (DLBS) und Kompensation (DK) (Trost, 2011). Die Existenz vielfältiger Strategien führt erwiesenermaßen zu einer erfolgreichen Bewältigung des Studiums. Aus diesem Grund weisen auch die generierten Items eine hohe Strategievielfalt auf. Ein derart breit angelegtes Inventar führt dazu, dass das Konstrukt insgesamt heterogen ist und sehr hohe Reliabilitätswerte kaum zu erwarten sind.

\section{Die Validierungsinstrumente}

Zur Validierung wird sowohl auf die (1) Konstrukt- als auch die (2) Kriteriumsvalidierung Bezug genommen. Zentrales Instrument zur Prüfung der Messgültigkeit ist das themenübergreifende Original-SOK-Inventar (Baltes et 
al., 1999) mit seinen Komponenten ES, O, LBS und K (je 3 Items). Zudem wurden zur Prüfung der Konstruktvalidität Inventare zur Messung der Persönlichkeit eingesetzt: Big-Five-Inventar (Lang \& Lüdtke, 2005) (15 Items) sowie 5 Items zur Handlungs- und Lageorientierung (Kuhl, o.J.) sowie auf Inventare zur Beschreibung von Strategieverhalten zurückgegriffen: Lernstrategien (Wild \& Schiefele, 1994) (9 Items), 12 Items aus dem TENFLEX (hartnäckige vs. flexible Zielanpassung) (Brandtstädter \& Renner, 1990) sowie 8 Items zur Selbstführung (Houghton \& Neck, 2002). Zur Kriteriumsvalidität wird auf Kriterien und Inventare zur Beschreibung von Erfolg Bezug genommen. Dabei sind der bisherige Notendurchschnitt im Studium und die Anzahl an abgesagten Klausuren Indikatoren für objektiven Erfolg. Subjektiver Erfolg wird mit den Inventaren Studienzufriedenheit (5 Items) (Westermann, 2006) und mit dem allgemeinen Wohlbefinden (Watson, Clark \& Tellegen, 1988) (10 Items) gemessen.

\section{Ergebnisse und Diskussion}

\section{Interne Konsistenz und Faktorenstruktur zur Bestimmung der Reliabilität}

Aufgrund der inkludierten Strategievielfalt kann DSOK als heterogenes latentes Merkmal bezeichnet werden. In Anlehnung an Lienert \& Raatz (1998, S. 267) soll daher ein Reliabilitätswert von Cronbachs $\alpha=0,70$ als akzeptabler Grenzwert angenommen werden. Die interne Konsistenz als Maß der Reliabilität beträgt: für DOSK: $\alpha=0,81$; DES: $\alpha=0,70$; DO: $\alpha=0,70$; DLBS: $\alpha=0,71$; DK: $\alpha=0,62$. Vergleichbare Werte ergeben sich auch für die Daten der 2. Validierungsstudie. Die Werte des SOK-Inventars von Baltes liegen hingegen deutlich niedriger zwischen $\alpha=0,53$ und $\alpha=0,78$ (Trost, 2011, S. 192).

Zur Bestimmung der Faktorenstruktur wurde auf Grundlage des Datensatzes der 2. Validierungsstudie eine explorative Faktorenanalyse gerechnet. Auf Basis der Hauptstudiendaten erfolgte im Anschluss die Prüfung der ermittelten Faktorenstruktur auf Eindimensionalität. Alle Berechnungen basieren auf Itemparcels anstelle von Einzelitems, da somit eine Varianzbündelung, eine erhöhte Annäherung an normalverteilte Variablen sowie eine Reduzierung der Modellkomplexität ermöglicht wird (Hoyle, 1999, S. 254).

Die Ergebnisse der EFA können mit einer Erklärungsvarianz von 65,75\% und einer deutlichen 4-Faktoren-Einfachstruktur (kaum Nebenladungen) als gut bewertet werden (unrotierte Eigenwerte: 4,003 (DES), 1,781 (DO), 1,277 (DLBS), 0,829 (DK)). Auch die Fit-Indizes der CFA: CMIN = 928,973; df = $344 ; \mathrm{p}=0,000 ; \mathrm{CMIN} / \mathrm{df}=2,191 ; \mathrm{GFI}=0,943 ; \mathrm{AGFI}=0,911 ; \mathrm{CFI}=0,941$; RMSEA $=0,063$; LO $90=0,047$, HI $90=0,080$; Bollen-Stine Bootstrap p = 0,002 sind als gut bis akzeptabel zu bewerten (Brown \& Cudeck, 1993, S. 
144; Byrne, 2001, S. 82-83). Anhand der Eigenwerte der EFA sowie der Faktorladungen der Subkomponenten auf DSOK $(\mathrm{DES}=0,82$; DO $=1,01$; DLBS $=0,16$ und DK $=0,55)$ in der Second-Order CFA zeigt sich die hohe Bedeutsamkeit der nicht von Verlust betroffenen Komponenten (DES und DO) am latenten Konstrukt.

\section{Konstruktvalidität}

Zur Prüfung der Konstruktvalidität wurden konvergente sowie divergente Konstrukte herangezogen. Die Konvergenzvalidität mit Hilfe von MultiTrait-Multi-Method (MTMM)-Analysen identifiziert konvergente und divergente Varianzanteile eines Konstrukts (Campbell \& Fiske, 1959), d.h. sie separiert zwischen der Varianz, die auf den Trait (z.B. elektive Selektion) (= konvergent) und der, die auf die Messmethode (gemessen mit dem bereichsübergreifenden SOK oder dem domänenspezifischen DSOK) (divergent) zurückzuführen ist. Die Konvergenzvalidität ist dann erfüllt, wenn die TraitInterkorrelationen (z.B. DES mit ES) höher ausfallen als die MethodenInterkorrelation (z.B. DES mit O).

Abbildung 1: Standardisierte Lösung für das Strukturgleichungsmodell CTCM-1 $(R S ; N=297):$ Fit-Indizes: $C M I N=27,67 ; d f=10 ; p=0,002$; $C M I N / d f=2,767 ; G F I=0,98 ; A G F I=0,92 ; C F I=0,97 ; R M S E A=$ 0,077; LO90 =0,04, HI90 =0,11; Bollen-Stine Bootstrap $p=0,011$

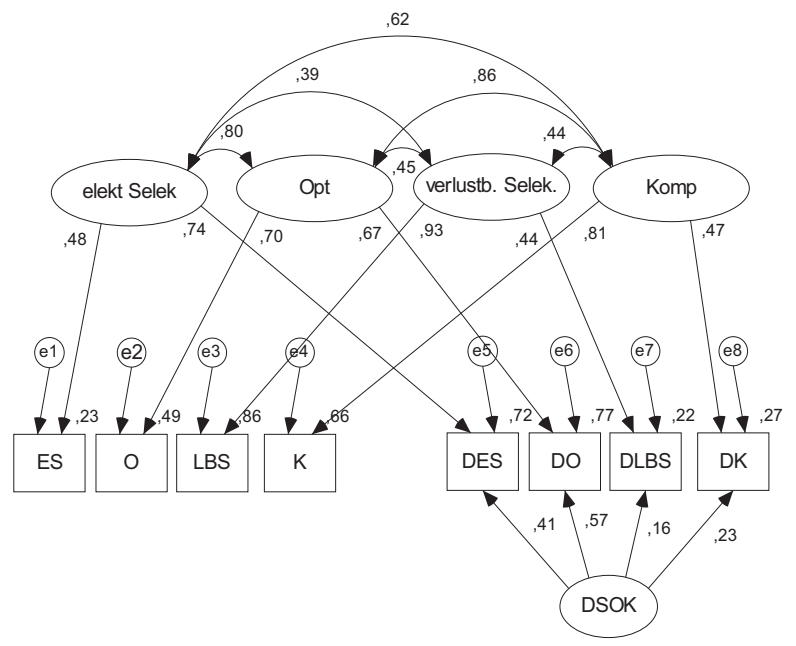


In Abb. 1 ist das - für die vorliegende Struktur identifizierbare - CTCM-1 Modell (Eid, 2000) einer kausalanalytischen MTMM-Matrix abgebildet. Da die DSOK-Trait-Ladungen (konvergente Varianz): $\mathrm{DES}=0,74 ; \mathrm{DO}=0,67$; DLBS $=0,44$ und $\mathrm{DK}=0,47$ höher sind als die jeweils korrespondierenden Methoden-Ladungen (divergente Varianz): $\mathrm{DES}=0,41 ; \mathrm{DO}=0,57 ; \mathrm{DLBS}=$ 0,16 und $\mathrm{DK}=0,23$, kann Konvergenzvalidität angenommen werden.

Des Weiteren wurden minderungskorrigierte Korrelationen von DSOK mit konvergenten und divergenten Persönlichkeits- und Strategieinventaren geprüft (Tab. 1). Insgesamt 27 der 40 Korrelationen (fett markiert) sind erwartungskonform. Auffällig ist die Komponente DLBS, die insgesamt wenig signifikante Zusammenhänge aufzeigt.

Tabelle 1: Minderungskorrigierte Korrelationen zur Prüfung der konvergenten und diskriminanten Validität mit verwandten Konstrukten zur Persönlichkeit und zum Strategieverhalten

\begin{tabular}{|c|c|c|c|c|c|c|c|}
\hline & $\begin{array}{l}\text { Validierungs- } \\
\text { konstrukte }\end{array}$ & $\begin{array}{l}\text { Cron- } \\
\text { bachs } \alpha\end{array}$ & DES & DO & DLBS & DK & DSOK \\
\hline \multirow{3}{*}{$\begin{array}{l}\text { Persön- } \\
\text { lichkeit }\end{array}$} & \multirow{3}{*}{$\begin{array}{l}\text { Extraversion } \\
\text { Gewissen- } \\
\text { haftigkeit } \\
\text { Offenheit } \\
\text { LO }\end{array}$} & 0,881 & 0,044 & $0,171^{* *}$ & 0,039 & $0,292^{\star *}$ & $0,216^{* *}$ \\
\hline & & 0,625 & $0,377^{* *}$ & $0,251^{* *}$ & $-0,065$ & 0,047 & $0,216^{* *}$ \\
\hline & & $\begin{array}{l}0,82 \\
1\end{array}$ & $\begin{array}{l}0,093 \\
0,220^{\star *}\end{array}$ & 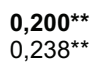 & $\begin{array}{l}0,074 \\
\mathbf{0 , 0 6 3}\end{array}$ & $\begin{array}{l}0,325^{\star *} \\
0,082\end{array}$ & $\begin{array}{l}\mathbf{0 , 2 7 1 ^ { * * }} \\
0,211^{* *}\end{array}$ \\
\hline $\begin{array}{l}\text { Stra- } \\
\text { tegie- } \\
\text { verhal- } \\
\text { ten }\end{array}$ & $\begin{array}{l}\text { Lernstrategien } \\
\text { Selbstführung } \\
\text { TEN } \\
\text { FLEX }\end{array}$ & $\begin{array}{l}0,659 \\
0,742 \\
0,749 \\
0,698\end{array}$ & $\begin{array}{l}0,444^{* *} \\
0,513^{* *} \\
0,381^{* *} \\
0,108\end{array}$ & $\begin{array}{l}0,505^{\star *} \\
0,450^{\star *} \\
0,382^{\star *} \\
0,084\end{array}$ & $\begin{array}{l}0,106 \\
\mathbf{0 , 1 5 4} \\
-\mathbf{0 , 1 4 8} \\
0,011\end{array}$ & $\begin{array}{l}0,353^{* *} \\
0,322^{* *} \\
0,307^{\star *} \\
0,104\end{array}$ & 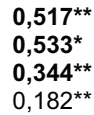 \\
\hline
\end{tabular}

Anmerkungen: TEN = tenacious goal pursuit; FLEX = flexible goal adjustment; LO = Lageorientierung; 2-seitige Pearson Korrelationen; ${ }^{*}=$ signifikant auf $0,05 \%$-Niveau; ${ }^{* *}=$ signifikant auf $0,01 \%$ Niveau; fett markierte Korrelationen = erwartungskonforme Korrelationen. 
Tabelle 2: Übereinstimmungskorrelationen DSOK mit objektiven und subjektiven Erfolgsfaktoren

\begin{tabular}{|c|c|c|c|c|c|}
\hline & DES & DO & DLBS & DK & DSOK \\
\hline \multicolumn{6}{|l|}{ objektive Erfolgsmaße } \\
\hline bisheriger Notenschnitt & $-0,141^{*}$ & $-0,011$ & 0,054 & 0,080 & $-0,008$ \\
\hline Anzahl abgesagter Klausuren & $-0,101$ & $-0,032$ & $0,154^{*}$ & 0,053 & 0,042 \\
\hline \multicolumn{6}{|l|}{ subjektive Erfolgsmaße } \\
\hline $\begin{array}{l}\text { Wohlbefinden } \\
(\text { Cronbachs } \alpha=0,86)\end{array}$ & $0,406^{* *}$ & $0,396^{* *}$ & $0,155^{*}$ & $0,332^{* *}$ & $0,480^{* *}$ \\
\hline $\begin{array}{l}\text { Studienzufriedenheit } \\
\text { (Cronbachs } \alpha=0,86)\end{array}$ & $0,356^{* *}$ & $0,190^{* *}$ & 0,064 & $0,138^{*}$ & $0,255^{\star *}$ \\
\hline
\end{tabular}

Anmerkungen: Pearsonsche Korrelationen, 2-seitig; ${ }^{*}=$ signifikant auf $0,05 \%-N i v e a u ;{ }^{* *}=$ signifikant auf 0,01\%-Niveau; fett markierte Korrelationen = erwartungskonforme Korrelationen.

\section{Kriteriumsvalidität}

Wie bereits aus der vorliegenden Literatur bekannt, weist die Anwendung von SOK- (bzw. hier DSOK-)Strategien häufig Übereinstimmungen mit subjektiven, nicht jedoch mit objektiven Erfolgsmaßen auf. Dies spiegelt sich auch in Tab. 2 (fett markierte Korrelationen) wieder. Auffällig ist erneut die Komponente DLSB, die wenig bzw. geringe Übereinstimmung mit positivem Erfolg zeigt. Von daher kann die Vermutung angestellt werden, dass die Anwendung von DLBS-Strategien nicht als adaptiv, also erfolgsförderlich wahrgenommen wird.

\section{Zusammenfassung und Ausblick}

Zusammenfassend lässt sich festhalten, dass studienbezogene Selbstregulationsstrategien zur Bewältigung typischer Studienprojekte (unter Berücksichtigung konkurrierender Lebensprojekte) mit Hilfe des neu entwickelten domänenspezifischen Instrumentes DSOK gemessen werden können. Die internen Konsistenzen übersteigen fast alle den vorher definierten Grenzwert von 0,7. Die explorativ identifizierte und dem theoretischen Modell entsprechende 4-Faktorenstruktur konnte mit einer Konfirmatorischen Analyse repliziert werden. Die Validität zeigt grundsätzlich erwartungskonforme Ergebnisse. Die Vermutung, kaum relevante Beziehungen von DSOK zu objektiven Erfolgsmaßen aufzeigen zu können, bestätigte sich. Es wird jedoch davon ausgegangen, dass eine höhere Zufriedenheit/ ein höheres Wohlbefinden langfristig den Umgang mit alläglichen Herausforde- 
rungen steigern kann und sich somit auch objektiv höherer Erfolg generieren lässt. Um diese Vermutung empirisch abzusichern, bedürfte es allerdings eines längeren Untersuchungszeitraums mit mehreren Messzeitpunkten.

Bei nahezu allen durchgeführten Analysen erwies sich die DSOKKomponente der domänenspezifischen verlustbasierten Selektion als durchgängig auffällig, d.h. es zeigen sich vielfach Effekte, die nicht den theoretischen Modell-Erwartungen entsprechen. Zielrevision (als zentrale DLBSStrategie) im Studienkontext scheint in einer durch Leistung und Exzellenz gekennzeichneten Gesellschaft ein Eingeständnis von Schwäche und demnach ein kulturelles Tabu zu sein. So lässt sich die Vermutung äußern, dass die Entwicklung von Selbstmanagementstrategien unterschiedlich verläuft und insbesondere mit Blick auf Strategien der flexiblen Zielanpassung in Verlustsituationen differenzierte Trainings für unterschiedliche „Persönlichkeiten" notwendig werden.

\section{Literatur}

Abele, A. E. \& Wiese, B. S. (2008). The Nomological Network of Self-Managment Strategies and Career Success. Journal of Occupational and Organizational Psychology, 81 (4), pp. 733-749.

Baethge, M., Achtenhagen, F., Arends, L., Babic, E., Baethge-Kinsky, V. \& Weber, S. (2006). PISA-VET. Stuttgart: Steiner.

Baltes, P. B. \& Baltes, M. M. (1989). Optimierung durch Selektion und Kompensation. Ein psychologisches Modell erfolgreichen Alterns. Zeitschrift für Pädagogik, 35 (1), S. 85-105.

Baltes, P. B., Baltes, M. M., Freund, A. M. \& Lang, F. R. (1999). The Measurement of Selection, Optimization, and Compensation (SOC) by Self Report: Technical Report 1999. Materialien aus der Bildungsforschung, Bd. 66. Berlin: MaxPlanck-Institut für Bildungsforschung.

Brandtstädter, J. \& Renner, G. (1990). Tenacious Goal Pursuit and Flexible Goal Adjustment. Explication and Age-Related Analysis of Assimilative and Accommodative Strategies of Coping. Psychology and Aging, 5 (1), pp. 58-67.

Brown, M. \& Cudeck, R. (1993). Alternative Ways of Accessing Equation Model Fit. - Testing Structural Equation Models. In K. A. Bollen (Hrsg.), Testing structural equation models. Sage focus editions: Bd. 154. (S. 136-162). Newbury Park: Sage.

Buttler, F. (1992). Tätigkeitslandschaft bis 2010. In F. Achtenhagen \& E. G. John (Hrsg.), Mehrdimensionale Lehr-Lern-Arrangements. Innovationen in der kaufmännischen Aus- und Weiterbildung. (S. 162-182). Wiesbaden: Gabler Verlag.

Byrne, B. M. (2001). Structural equation modeling with AMOS. Basic Concepts Applications and Programming. Multivariate applications series. Mahwah, NJ [et al.]: Erlbaum. 
Campbell, D. T. \& Fiske, D. W. (1959). Convergent and Discriminant Validation by Multitrait-Multimethod Matrix. Psychological Bulletin, 56, pp. 81-105.

Eid, M. (2000). A Multitrait-Multimethod Model with Minimal Assumptions. Psychometrika, 65, pp. 241-261.

Freund, A. M. (2007). Selektion, Optimierung und Kompensation im Kontext persönlicher Ziele. Das SOK-Modell. In J. Brandtstädter \& U. Lindenberger (Hrsg.), Entwicklungspsychologie der Lebensspanne. Ein Lehrbuch. 1. Aufl. (S. 367388). Stuttgart: Kohlhammer.

Freund, A. M. \& Baltes, P. B. (2000). The Orchestration of Selection, Optimization, and Compensation: An Action-Theoretical Conceptualization of a Theory of Developmental Regulation. In W. J. Perrig, A. Grob \& A. Flammer (Hrsg.), Control of Human Behavior, Mental Processes, and Consciousness. (S. 35-58). Mahwah, N.J, London: Erlbaum.

Freund, A. M. \& Baltes, P. B. (2002). Life-Management Strategies of Selection, Optimization and Compensation: Measurement by Self-Report and Construct Validity. Journal of Personality and Social Psychology, 82 (4), pp. 642-662.

Gusy, B., Lohmann, K. \& Drewes, J. (2010). Burnout bei Studierenden, die einen Bachelor-Abschluss anstreben. Prävention und Gesundheitsförderung Volume 5, Number 3,. Prävention und Gesundheitsförderung, 5 (3), S. 271-275.

Heublein, U., Hutzsch, C., Schreiber, J., Sommer, D. \& Besuch, G. (2010). Ursachen des Studienabbruchs in Bachelor- und in herkömmlichen Studiengängen. Ergebnisse einer bundesweiten Befragung von Exmatrukulierten des Studienjahres 2007/08. Gefunden am 10.06.2010 unterhttp://www.his.de/pdf/21/studienabbruch ursachen.pdf

Hochschulrektorenkonferenz (2004). Bologna Reader, Texte und Hilfestellungen zur Umsetzung der Ziele des Bologna-Preozesses an deutschen Hochschulen". Gefunden am 16.06.2010 unter http://www.hrk.de/bologna/de/Bologna_Reader_gesamt.pdf

Houghton, J. D. \& Neck, C. P. (2002). The Revised Self-Leadership Questionnaire: Testing a Hierarchical Factor Structure for Self-Leadership. Journal of Managerial Psychology, 17 (8), pp. 672-691.

Hoyle, R. H. (1999). Statistical Strategies for Small Sample Research. Thousand Oaks [u.a.]: Sage.

Kuhl, J. (о.J.). Kurzanweisung zum Fragebogen HAKEMP 90. Gefunden am 17.01.2010 unter http://www.psychologie.uzh.ch/fachrichtungen/motivation/ Studium/lehre/alt/ws0506/methodenIWS0506/hakemp90.pdf

Lang, F. R. \& Lüdtke, O. (2005). Der Big-Five-Ansatz der Persönlichkeitsforschung. Instrumente und Vorgehen. In S. Schumann \& H. Schoen (Hrsg.), Persönlichkeit. Eine vergessene Größe der empirischen Sozialforschung. 1. Aufl. (S. 29-40). Wiesbaden: VS Verlag der Sozialwissenschaften.

Lienert, G. A. \& Raatz, U. (1998). Testaufbau und Testanalyse (6. Aufl.). Weinheim: Psychologie Verlags Union.

Lindenberger, U. (2006). Erwachsenenalter und Alter. In R. Oerter \& L. Montada (Hrsg.), Entwicklungspsychologie. (S. 350-391). Weinheim: Beltz Verlag.

OECD (2008). Definition und Auswahl von Schlüsselkompetenzen. Zusammenfassung. Gefunden am 13.05.2008 unter http://www.portal-stat.admin.ch/deseco/deseco_ finalreport_summary.pdf 
Picot, A., Reichwald, R. \& Wigand, R. T. (2001). Die grenzenlose Unternehmung. Wiesbaden: Gabler Verlag.

Staudinger, U. M., Marsiske, M. \& Baltes, P. B. (1995). Resilience and reserve capacity in later adulthood: Potentials and limits of development across the life span. In D. Cicchetti \& D. Cohen (Hrsg.), Developmental psychology. Bd. 2. (S. 801847). New York: John Wiley \& Sons Inc.

Trost, S. (2011). Studienbezogene Selbstregulation. Eine Adaptation des Metamodells "Selektion, Optimierung und Kompensation". Berufliche Bildung im Wandel, Bd. 14. Frankfurt am Main: Lang (Univ., Diss.-München, 2010.).

Watson, D., Clark, L. A. \& Tellegen, A. (1988). Development and Validation of Brief Measures of Positive and Negative affect: The PANAS Scale. Journal of Personality and Social Psychology, 54 (6), pp. 1063-1070.

Weber, S. (2005). Kompetenz und Identität als Konzepte beruflichen Lernens über die Lebensspanne. In P. Gonnon, F. Klauser, R. Nickolaus \& R. Huisinga (Hrsg.), Kompetenz, Kognition und neue Konzepte der beruflichen Bildung. (S. 9-23). Wiesbaden: VS Verlag der Sozialwissenschaften.

Westermann, R. (2006). Studienzufriedenheit. In D. H. Rost (Hrsg.), Handwörterbuch der Pädagogischen Psychologie. (S. 756-763). Weinheim: Beltz Verlag.

Wiese, B. S. (2000). Berufliche und familiäre Zielstrukturen. Ein Anwendungsbeispiel zum Meta-Modell der Selektiven Optimierung mit Kompensation. Münster: Waxmann Verlag (Dissertation).

Wiese, B. S. \& Schmitz, B. (2002). Studienbezogenes Handeln im Kontext eines entwicklungspsychologischen Meta-Modells. Zeitschrift für Entwicklungspsychologie und Pädagogische Psychologie, 34 (2), S. 80-94.

Wiese, B. S., Freund, A. M. \& Baltes, P. B. (2000). Selection, Optimization, and Compensation: An Action-Related Approach to Work and Partnership. Journal of Vocational Behavior, 57 (3), pp. 273-300.

Wild, K.-P. \& Schiefele, U. (1994). Lernstrategien im Studium: Ergebnisse zur Faktorenstruktur und Reliabilität eines neuen Fragebogens. Zeitschrift für Differentielle und Diagnostische Psychologie, 15 (4), S. 185-200. 


\section{Lernen und Arbeiten in Balance? Vereinbarkeitsstrategien von Beschäftigten in wissensintensiven Branchen}

Julia K. Müller, Rita Meyer

\section{Lernen und Arbeiten in der Wissensgesellschaft}

Arbeit in der Wissensgesellschaft ist zunehmend durch wissensbasierte Tätigkeiten gekennzeichnet, die eine permanente Aktualisierung sowie die kreative und innovative Nutzung von Wissen erfordern (vgl. Staudt 2002; Wilkens 2004). Die Arbeitsaufgaben in der Wissensarbeit sind durch kognitive Aktivitäten geprägt und bringen i. d. R. immaterielle Leistungen hervor. Diese basieren auf dem Sammeln, Bewerten und Bündeln von Informationen, durch welche neues Wissen erzeugt oder bestehendes Wissen modifiziert wird. Die Arbeitsprozesse zeichnen sich durch einen hohen Komplexitätsgrad aus, denn die Arbeitsabläufe sind kaum standardisierbar (wenige Routinen) und weisen Planungsunsicherheit sowie große Handlungsspielräume auf. Neuartige Aufgabenstellungen erfordern von den Beschäftigten ein hohes $\mathrm{Ma} ß$ an Fertigkeiten, Kompetenzen und ständig neuem Wissen (vgl. Davenport et al. 1996; Willke 1998; North 2008) und damit eine permanente (Weiter-)Entwicklung der umfassenden beruflichen Handlungskompetenz (vgl. Dehnbostel 2005; 2007).

Diesen Herausforderungen begegnen Wissensarbeiter durch permanentes selbstgesteuertes Lernen. Dabei übernimmt das Erfahrungslernen einen elementaren Stellenwert, denn durch das Planen und Entwickeln von Strategien, die Analyse von vielfältigen Sonderfällen und Ausnahmen und die Kombination von neuen Informationen mit bestehendem Wissen, werden beständig neue Erfahrungen gewonnen. Das Lernen aus Erfahrungen erfolgt in der Wissensarbeit dabei häufig implizit, d. h. unbewusst (vgl. Pfiffner/Stadelmann 1999; North 2008) und im Prozess der Arbeit. Das selbstgesteuerte Lernen ist der Wissensarbeit insofern immanent. Um die benötigten Fähigkeiten, Fertigkeiten, das Wissen und die berufliche Handlungskompetenz aktuell zu halten, zu revidieren oder auch neu zu erwerben, sind Beschäftigte in der Wissensarbeit gefordert, die immer komplexer werdenden Informationen sinnvoll zu verdichten und so auf das Elementare hin zu reduzieren. Dies erfordert einen hohen Kommunikations- und Kooperationsaufwand sowie eine besondere Einstellung zum Lernen. Wissensarbeiter haben in der Regel eine relativ autonome Arbeitssituation. Dies fördert einerseits 
die Bereitschaft überhaupt zu lernen, andererseits eröffnen solche Konstellationen die Möglichkeit, Lern- und Arbeitsprozesse aktiv zu gestalten. Beschäftigte in der Wissensarbeit sind insofern mit besonderen Lernerfordernissen konfrontiert: Sie müssen sich sowohl täglich mit allem Lernbaren auseinandersetzen, als auch eine bewusste und persönliche Systematisierung der eigenen Arbeitserfahrungen vornehmen (vgl. Pfiffner/ Stadelmann 1999). Eine bewusste Reflexion der eigenen Arbeitserfahrungen und des erworbenen Wissens ist dabei unabdingbar.

Obwohl Wissensarbeit in ihrer Struktur grundsätzlich förderliche Voraussetzungen für das Lernen mit sich bringt (z. B. durch große Handlungsspielräume), resultieren daraus auch Lernhindernisse: Studien verweisen auf eine mangelnde Wechselwirkung zwischen formellem und informellem Lernen und die Uneindeutigkeit zwischen Arbeits- und Lernorganisationsformen (vgl. Harz 2004; Rohs 2007). Häufig bleibt im Arbeitsalltag zudem nicht ausreichend Zeit für bewusstes Lernen. Die Zeit als beeinträchtigender Faktor sowie das Paradigma der betrieblichen Verwertbarkeit des Lernens kristallisieren sich als zentrale Herausforderungen im Zusammenhang mit Lernen und Arbeiten in wissensintensiven Branchen heraus (vgl. Salman 2009). Mit zunehmendem Zeit- und Lerndruck steigt auch das Risiko, die Arbeitsanforderungen nicht mehr adäquat zu erfüllen. Hier entsteht in der zeitlichen Dimension eine Imbalance von Arbeits- und Lernprozessen, die das notwendige Wechselverhältnis von Arbeiten und Lernen auch in pädagogischer Hinsicht negativ beeinflussen kann.

Aus erziehungswissenschaftlicher Sicht geht es bei diesem Wechselverhältnis immer auch um die Interdependenz von individueller Kompetenzund betrieblicher Organisationsentwicklung (vgl. Dehnbostel 2005, 2007; Molzberger 2007; Meyer 2011). Die berufs- und betriebspädagogische Relevanz ergibt sich explizit, weil das Lernen des Individuums wechselseitig mit der Entwicklung der Arbeitsprozesse, des Arbeitsplatzes und des Unternehmens verknüpft ist. Megatrends, wie die Globalisierung und die Entwicklung der Informations- und Kommunikationstechnologien, verstärken in den wissensintensiven Branchen den Innovations- und Wettbewerbsdruck, dem sowohl Unternehmen als auch Beschäftigte ausgesetzt sind. Daher sind in der betrieblichen Praxis beide Seiten (Organisation und Individuum) mit der Notwendigkeit der Gestaltung von kontinuierlichen Lern- und Entwicklungsprozessen konfrontiert.

In verschiedenen Disziplinen finden sich eine Vielzahl an theoretischen Beschreibungen und Analysen zum Zusammenwirken von Kompetenz- und Organisationsentwicklung (vgl. Frei 1985; Baitsch 1986; Bartels 2001; Voigt et al. 2005; Molzberger 2007). Die Erkenntnisse aus diesen empirischen Studien zeigen, dass es in der Wissensarbeit kaum organisierte formale Weiterbildung gibt und dass informelles und selbstorganisiertes Lernen deutlich dominieren (vgl. Heyse/Erpenbeck/Michels 2002; Dehnbostel/Molzberger/ 
Overwien 2003). Allerdings wurden die Zusammenhänge und Wechselwirkungen von Kompetenz- und Organisationsentwicklung bislang kaum theoretisch und empirisch untersucht. Ein Desiderat stellt zudem Forschungen zu individuellen Lern- und Vereinbarkeitsstrategien der Bereiche Arbeiten, Lernen und Leben dar. An dieser Forschungslücke setzt $u$. a. das Forschungsprojekt „Arbeiten - Lernen - Leben in der Wissensarbeit“ (ALLWiss) an, in welchem nach den Faktoren einer gelungenen Work-Lern-Life-Balance gefragt wird.

\section{Forschungsprojekt ALLWiss: Arbeiten - Lernen - Leben in der Wissensarbeit}

Ziel des interdisziplinären Forschungsprojektes ${ }^{1}$ ist die empirische Beschreibung und Analyse spezifischer Work-Learn-Life-Balance-Situationen. Ermittelt werden insbesondere Faktoren von Belastungen und Ressourcen, die diese Situationen auf individueller, sozialer und organisationaler Ebene kennzeichnen. In Zusammenarbeit mit Unternehmen sollen ausgehend von der empirischen Untersuchung Work-Learn-Life-Balance-Modelle entwickelt und als Instrumente für verschiedene Handlungsfelder der betrieblichen Praxis entwickelt und erprobt werden.

Theoretische Ausgangspunkte sind Konzepte zur Verknüpfung individueller Lernprozesse mit betrieblichen Organisationsprozessen, die neben der Förderung der wirtschaftlichen Entwicklung auch die individuelle Kompetenzentwicklung der Beschäftigen berücksichtigen (vgl. Dehnbostel 2005, 2007; Meyer 2006, 2011). Dabei finden auch Konzepte der beruflichen Handlungskompetenz sowie der strukturellen Reflexivität und der Selbstreflexivität Berücksichtigung (vgl. Lash 1996; Dehnbostel 2005, 2007).

In dem Forschungsprojekt wird folgenden Fragen nachgegangen: (1) Welche individuellen und organisationalen Ressourcen für informelles Lernen lassen sich feststellen? (2) Welche Faktoren lassen sich im Bereich Wissensarbeit identifizieren, die eine Balance zwischen Arbeiten, Lernen und Leben erschweren bzw. befördern? (3) Welche Strategien entwickeln Beschäftigte, um Lern- und Arbeitsanforderungen miteinander zu vereinbaren?

1 Das Forschungsprojekt ALLWiss wird vom Bundesministerium für Bildung und Forschung (BMBF) sowie aus Mitteln des Europäischen Sozialfonds (ESF) für die Laufzeit vom 01. August 2009 bis zum 30. April 2013 gefördert. Der Förderschwerpunkt gehört zum BMBFForschungs- und Entwicklungsprogramm Arbeiten - Lernen - Kompetenzen entwickeln. Innovationsfähigkeit in einer modernen Arbeitswelt - www.allwiss.de 


\section{Untersuchungssample und methodisches Vorgehen}

Das Untersuchungssample der Studie umfasst zwei Praxispartner- und vier Valuepartnerunternehmen ${ }^{2}$, die der IT-Branche zugeordnet werden können. Die Datenerhebung gliedert sich in zwei Phasen: Einen qualitativen Teil, bei dem in zwei Praxispartner- und drei Valuepartnerunternehmen qualitative leitfadengestützte Einzel- und Gruppeninterviews durchgeführt wurden. Im zweiten Teil erfolgte eine quantitative Online-Erhebung bei zwei Praxispartner- und vier Valuepartnerunternehmen. Ziel der qualitativen Gruppeninterviews $(\mathrm{N}=166)$ war einerseits ein gegenseitiges Kennenlernen als vertrauensbildende Maßnahme, andererseits wurden bereits erste Belastungen, Ressourcen und Bewältigungsstrategien vor dem Hintergrund der Trias Arbeit - Lernen - Leben reflektiert. Die leitfadengestützten Einzelinterviews $(\mathrm{N}=73)$ erfassten anschließend die individuelle Sicht der Befragten in Bezug auf das persönliche Umfeld sowie das Arbeitsumfeld. Die Interviews wurden vollständig transkribiert und durch eine Kombination von zusammenfassender und strukturierender Inhaltsanalyse ausgewertet (vgl. Mayring 2002, 2008). Die quantitative Online-Befragung wurde auf Basis der im qualitativen Teil erhobenen Daten konzipiert und umfasste sowohl etablierte Skalen (fünfstufige Ratingskala) als auch selbst entwickelte Items zu den Themenfeldern Anforderungen/Belastungen, Ressourcen und Handlungsstrategien/Maßnahmen. Ziel der quantitativen Untersuchung war eine spezifischere Analyse der fokussierten Schnittstellen und Wechselwirkungen zwischen Lernen und Arbeiten in der Wissensarbeit.

Das Untersuchungsdesign folgt einem abduktiven, handlungstheoretischen Ansatz. Aus dieser Perspektive wird ein dialogisches bzw. diskursives Verhältnis von Theorie und Empirie angestrebt, wobei die Theorie gegenüber der Empirie eine erklärende, reflexionsfördernde und somit aufklärende Funktion übernimmt (vgl. Meyer 2006). Dabei wurden die Fragestellungen ebenso wie die Erhebungsmethoden theoriebasiert erarbeitet. Traten Widersprüche oder Unstimmigkeiten von Empirie und Theorie auf, so führten diese nicht zum Bruch mit den Ausgangsannahmen, sondern zur theoretischen Reflexion und Weiterentwicklung der zu Grunde gelegten Ansätze (vgl. Kelle 1994; Reichertz 2003).

2 Die Unterscheidung von Praxis- und Valuepartnerunternehmen geht auf die Dauer der Teilnahme sowie auf die inhaltliche Einbindung im Forschungsprojekt zurück. Die Valuepartnerunternehmen standen in der Erhebungsphase zusätzlich für die qualitative Befragung und die quantitative Online-Erhebung zur Verfügung. Die Praxispartner sind darüber hinaus auch aktiv an der Entwicklung der Instrumente zur Verbesserung der Work-Learn-LifeBalance beteiligt. 


\section{Erste Ergebnisse zur Vereinbarkeit von Arbeiten und Lernen in der Wissensarbeit}

\section{(1) Individuelle und organisationale Ressourcen für informelles Lernen}

Mit Blick auf die Ressourcen für informelles Lernen lässt sich auf der Ebene des Individuums zunächst konstatieren, dass die individuellen Ressourcen die Kompetenzen der Beschäftigten darstellen, die diese während ihres Arbeitslebens erworben haben. Der entscheidende Erfolgsfaktor ist das "Up-to-date" bleiben - also das Lernen. Dies gilt hinsichtlich der Produkte und Dienstleistungen, die erstellt und verkauft werden, aber auch bezogen auf das Marktgeschehen und die technische Entwicklung. Lernen wird von den Befragten als grundsätzlich positiv und als spannende Herausforderung beschrieben. Sie verfügen insofern per se über eine hohe Lernbereitschaft und organisieren ihr Lernen eigenständig. Offenheit und die Neugierde für Neues stellen für sie Selbstverständlichkeiten beim Lernen und Arbeiten in der IT-Welt dar: "Man sollte halt einfach für die Weiterentwicklungen aufgeschlossen sein" (02KFH, S. 7). Insbesondere den zieloffenen und flexiblen Umgang mit dem durch Unsicherheit und Ungewissheit geprägten Lernen empfinden die Befragten als Ressource und Motivationsquelle. Dies bestätigen auch die quantitativen Daten der Kategorie Problemlösen $(\mathrm{M}=3.76, \mathrm{SD}=.84)$.

Auf organisationaler Ebene stellen die Ressourcen die Arbeits- und Lerninfrastruktur des Unternehmens dar. Die Arbeit in den untersuchten Unternehmen ist projektorientiert gestaltet und eröffnet die Möglichkeit, problemhaltige Arbeitsaufgaben durch einen großen Handlungsspielraum eigenständig und differenziert zu bewältigen. Die Ergebnisse der Studie weisen darauf hin, dass die projektförmig organisierte Arbeit, die Nutzung und Entwicklung der individuellen Ressourcen bzw. Kompetenzen fördert. Dies gilt insbesondere für die Teamarbeit - als Teil projektförmig organisierter Arbeit - welche als eine zentrale Voraussetzung für Kommunikationsprozesse wahrgenommen wird. Diese Arbeitsformen weisen interaktive Gestaltungselemente, wie z. B. Teamsitzungen, gemeinsames Brainstorming, etc., auf, die nach Einschätzung der Befragten die kommunikative und soziale Ebene des Arbeitens und Lernens unterstützen und ein Lernen auch aus den Erfahrungen Anderer ermöglichen. Dies wird durch die Skala kollektives Arbeiten und Lernen in der quantitativen Befragung bestätigt $(\mathrm{M}=3.41$, SD $=.79$ ). Des Weiteren zeigen die Ergebnisse, dass Mitarbeitergespräche die Gelegenheit bieten, informelles Lernen anzustoßen, indem gemeinsam mit der Führungskraft über Bedürfnisse, Ziele und Entwicklungsmöglichkeiten auf beiden Seiten gesprochen und reflektiert werden:

"Bei diesen Mitarbeiterjahresgesprächen, da können wir auch Entwicklungsfelder angeben (...), die man sich vorstellen könnte, die einem dazu helfen können." (24SEW, S. 5). 
Festzuhalten ist also, dass schwierige, nicht nach einem bekannten Muster zu lösende Arbeitsaufgaben, ebenso wie hohe Gestaltungs- und Freiheitsgrade, anregend auf Beschäftigte in der Wissensarbeit wirken und kreative Energien frei setzen. Dies belegt auch die quantitative Erhebung mit der Kategorie $A u$ tonomie $(\mathrm{M}=3.58, \mathrm{SD}=.79)$.

\section{(2) Balance- und Imbalancefaktoren zur Vereinbarkeit von Arbeiten und Lernen}

In den befragten Unternehmen wurde deutlich, dass die Balance von Arbeiten und Lernen zunächst durch eine inhaltliche Gleichwertigkeit der beiden Bereiche bestimmt ist:

Bei mir ist das Lernen wirklich im Alltag. Ich lerne mit jeder Situation, die ich durchlebe, mein Tag ist niemals ähnlich oder gleich mit dem Tag davor oder einem Jahr davor. (18IMC, S. 4)

Als besonders prägend nennen die Befragten, dass sich Arbeits- und Lernanforderungen ergänzen und gegenseitig fördern. Die Arbeit selbst wird von den Mitarbeitern als Lernanforderung wahrgenommen. In der quantitativen Erhebung wurde diese Wahrnehmung bestätigt. Als Ergebnis kann festgehalten werden, dass die Arbeitstätigkeit überwiegend permanente Lernerfordernisse an die Beschäftigten stellt $(\mathrm{M}=3.63$ und $\mathrm{SD}=.92)$. Die gegenseitige Ergänzung stellt einen wichtigen Balance-Faktor zur Vereinbarkeit beider Domänen dar. Dadurch, dass die Arbeit selbst lernhaltig ist und der Arbeitsprozess als Lernprozess angesehen wird, werden Beschäftigte in der Wissensarbeit durch ihre alltäglichen Arbeitsaufgaben damit konfrontiert, zu lernen. Dies geschieht vor allem durch Ausprobieren und durch die Sammlung von Erfahrungen aber auch im kommunikativen Austausch untereinander.

Indem man einmal schlechte Erfahrungen gemacht hat oder gute Erfahrungen gemacht hat, weil man es einfach selbst mal ausprobiert hat und gesagt hat, 'ach das hat geklappt oder nicht geklappt', beim nächsten Mal würde ich es anders machen. (27PLW, S. 9)

Individuelle Erfahrungen werden diskutiert und von verschiedenen Seiten beleuchtet; durch gezieltes Nachfragen bei erfahrenen Kollegen wird eine gemeinsame Lösungsfindung vorangetrieben.

Oder indem man halt eben Kollegen fragt und sagt 'guck mal, ich habe das und das Problem, mit einem Kunden, der kommt da jetzt nicht voran und was machen wir denn da. Also dann heißt es auch schon Netzwerken, viele Leute kennen, die Nummer, fragen und keine Scheu haben zu fragen. (27PLW, S. 9) 
Die Befragten weisen der Kommunikation im Netzwerk eine entscheidende Rolle im Hinblick auf ihre Kompetenzentwicklung zu. Einerseits werden gezielt Kollegen als Experten aus bestimmten Fachgebieten in die eigene Arbeit miteinbezogen, andererseits auch Kunden als Spezialisten befragt und als Produzenten für Lernanstöße genutzt.

Im Endeffekt haben wir auch Weiterbildungen, weil es auch einfach der Kunde fordert. Das heißt auch der möchte, dass wir auf einem aktuellen Wissensstand sind. (02KFH, S. 8)

Neben den fördernden Faktoren zur Vereinbarkeit von Arbeiten und Lernen, verwiesen die Befragten auch auf balancehemmende Faktoren. Im Gegensatz zur oben genannten inhaltlichen Gleichwertigkeit der Bereiche Arbeiten und Lernen zeigt sich ein zeitliches Auseinanderfallen dieser beiden Bereiche.

Wir haben intern Seminare auch eben während der Arbeitszeit. Die Arbeit bleibt in dieser Zeit einfach liegen und muss dann nachgeholt werden (...). Und das zu vereinbaren, ist manchmal ein bisschen schwierig. (24SEW, S. 9)

Die Befragten führen dies zum einen auf eine mangelnde organisatorische und strukturelle Rahmung des Lernens, zum anderen auf die Ausrichtung des Lernens an ökonomischen Prinzipien zurück.

In der Zeit, wenn sie neue Dinge lernen, können sie nicht arbeiten! Weil da lernen sie ja! Das ist schon immer ein Problem seinem Vorgesetzten klar zu machen, okay, es ist hier jetzt einfach mal Zeit in was Neues zu investieren, sei es, dass man sich 14 Tage mal was Neues anschaut. Das ist schon ein Punkt, der schwierig ist. Weil 14 Tage kriegen sie nicht so ohne weiteres. Das sind 14 Tage, die sie nicht produktiv sind, sondern nur kosten. (05TMK, S. 23)

Bezogen auf formelle Lernprozesse beschreiben die Befragten ein Missverhältnis von Investition und Nachhaltigkeit. Lernen wird vielfach unter betriebswirtschaftlichen Prämissen verkürzt beurteilt und erscheint nur dann sinnvoll, wenn für das Unternehmen ein unmittelbarer Nutzen sichtbar wird. Durch die quantitative Erhebung wird diese Erkenntnis gestützt. Sie kommt zu dem Ergebnis, dass lediglich eine mittelmäßige $(\mathrm{M}=2.69, \mathrm{SD}=.95) \mathrm{Un}$ terstützung des Lernens im Arbeitsalltag durch die Unternehmen stattfindet.

Hervorzuheben ist, dass die Befragten implizit eine Unterscheidung von formellem und informellem Lernen treffen. Wurde in den Interviews allgemein nach ,, dem Lernen " gefragt, so liegen die Beschreibungen und Erklärungen der Befragten im Bereich des informellen Lernens und verweisen auf einen hohen Anteil dieser Lernform, die durch hohe Eigeninitiative und Selbstständigkeit geprägt ist. Obwohl dies als Anforderung seitens des Unternehmens gestellt wird, wird das permanente Lernen von den Mitarbeitern als positive Herausforderung erlebt. Von Schwierigkeiten berichten die Befragten im Bereich des formellen Lernens. Der geringe Institutionalisierungs- 
und Organisationsgrad dieses Lernens erweist sich für die Wissensarbeiter als Problem.

Ich würde mir eine Person wünschen, oder eine ganze Abteilung wünschen, die dann nichts anderes macht, wie das ganze Jahr über alle Funktionen im Haus, angefangen vom Chef, der muss genauso lernen, bis zum kleinsten Mitarbeiter in der Logistik alles im Blick hält. (02SGJ, S. 14)

In den untersuchten Unternehmen sind die Lernprozesse hochgradig individualisiert, es existieren kaum etablierte Lernkulturen und somit auch keine für Weiterbildung vorgesehenen Zeitfenster. Obwohl das Lernen nachgewiesenermaßen einen hohen Stellenwert einnimmt, fehlen unterstützende Strukturen. Durch die nicht vorhandenen pädagogischen Rahmungen des informellen Lernens entstehen strukturelle Schwierigkeiten: Im Arbeitsprozess bleibt häufig kaum Zeit für gezielte Recherchen, vertieftes Lesen oder einen detaillierten Erfahrungsaustausch. Das Lernen im Prozess der Arbeit kann so nur in einem geringen Maß gezielt und bewusst erfolgen. Dies wurde in der quantitativen Erhebung durch die Kategorie Lernbeeinträchtigung durch den Zeitdruck erhoben. Der Mittelwert von $\mathrm{M}=2.72(\mathrm{SD}=.99)$ weist auf eine mittelmäßige Beeinträchtigung des Lernens durch den Zeitdruck hin. Beide Lernformen - formell und informell - werden davon negativ beeinflusst und die Bereiche Lernen und Arbeiten, die in der Wissensarbeit eng miteinander verbunden sind, geraten in eine Imbalance. Dies wiederum erweist sich als dysfunktional zur Bewältigung der Arbeitsanforderungen.

\section{(3) Lern- und Vereinbarkeitsstrategien der Wissensarbeiter}

Um Lernen und Arbeiten in Balance zu halten, haben die Beschäftigten individuelle Strategien entwickelt, die es ihnen ermöglichen, die Anforderungen der beiden Bereiche miteinander zu vereinbaren. Beispielsweise richten sie sich selbstständig und kontinuierlich feste Lernzeitfenster ein. Darüber hinaus gestalten sie ihre Arbeitsabläufe integrativ und interdependent, indem sie Arbeitskollegen in eigene Tätigkeiten und Arbeitsabläufe einbinden. So werden auf informeller, kollegialer Ebene auch Vertretungsregelungen realisiert.

Wir hatten auch noch einen Kollegen, der sehr erfahren war, was so Projektmanagement betrifft, der hat mir auch ziemlich gut geholfen, wie man was strukturieren kann, also hat mir wirklich konkrete Tipps gegeben und auch Bücher empfohlen. (12UEW, S. 7)

Dies zeigen auch die Ergebnisse der quantitativen Befragung zu Arbeitszeitregelung und die damit in Zusammenhang stehende Möglichkeit, Lernzeiten selbstständig in den Arbeitsprozess einzuplanen $(\mathrm{M}=3.08)$. Die Standardabweichung von $\mathrm{SD}=1.13$ verweist jedoch darauf, dass es in den untersuch- 
ten Unternehmen auch zahlreich Beschäftigte gibt, die durch vergleichsweise restriktive Arbeitszeitmodelle nicht die Möglichkeit haben, sich ihre Lernzeiten einzuteilen.

Auch die selbstgewählte und bewusst vollzogene Entgrenzung der Bereiche Arbeiten, Lernen und Leben sowie die Unterstützung durch das private soziale Umfeld stellen zentrale Vereinbarkeitsstrategien dar.

Ich würde sagen sie (die Bereiche Arbeiten, Lernen, Leben) überschneiden sich ein bisschen, weil ich schon mal was heim nehme, wenn ich merke, ich muss da schnell fit werden, dann hole ich dann das Buch und dann muss ich es irgendwie noch am Wochenende oder am Abend lesen. Es kommt schon vor, es kommt jetzt nicht regelmäßig vor, sondern wirklich, wenn ich merke, ich brauche schnell Wissen. (06 SHE, S. 8)

Die Unternehmen unterstützen durch den Aufbau einer sozialen Infrastruktur (z. B. 360-Grad-Teams und flexible Arbeitszeitmodelle) integratives und kollegiales Arbeiten. Flexible Arbeitszeitmodelle sowie Mitarbeitergespräche, die von Seiten der Organisation angeboten werden, ermöglichen die bedarfsnahe Organisation des Lernens, jedoch unter der Voraussetzung, dass seitens der Mitarbeiter eine aktive Nutzung und gezielte Anwendung dieser Modelle erfolgt. Dies belegen auch die quantitativen Daten hinsichtlich regelmäßiger Feedbackgespräche mit der Führungskraft $(\mathrm{M}=3.07, \mathrm{SD}=1.29)$. Die große Standardabweichung zeigt allerdings, dass diese Gespräche nicht in allen Unternehmen gleich genutzt oder angeboten werden; zudem verweist dies auch auf unternehmensinterne Unterschiede.

\section{Zusammenfassung und Fazit}

Die empirischen Daten bestätigen, dass die Wissensarbeit durch große Aufgabenvielfalt und wenig Routinen gekennzeichnet ist. Damit gehen sowohl eine hohe Komplexität der Arbeitsabläufe und -prozesse als auch ein hoher Autonomiegrad bei der Ausführung der Arbeitsaufgaben einher. Die Generierung des neuen Wissens erfolgt hauptsächlich in kollegialen interaktiven Arbeitsformen (Projekt- und Teamarbeit) sowie schnell, vielfach ad hoc und dennoch priorisiert. Von organisationaler Seite werden Lernprozesse häufig nicht als solche wahrgenommen. Dies führt dazu, dass auf individueller Ebene zwar ein Lernen im Prozess der Arbeit stattfindet, jedoch keine (Lern-) Arrangements etabliert werden, die dieses Lernen als solches bewusst machen. Hier werden Potenziale für dauerhafte und nachhaltige Kompetenzentwicklung der Beschäftigten und die Entwicklung der Organisation verschenkt. Den besonderen Herausforderungen des Lernens in der Wissensarbeit (hohe Komplexität, Zeitdruck, fehlende Lernkultur etc.) begegnen die Wissensarbeiter, indem sie ihr Lernen in hohem Maße selbst organisieren 
und selbst steuern. Die individuellen Lern- und Vereinbarkeitsstrategien der Wissensarbeiter tragen zur Stärkung und Entfaltung der beruflichen Handlungskompetenz, zur individuellen Kompetenzentwicklung sowie zur Stabilisierung der Work-Learn-Life-Balance bei. Jedoch mangelt es an einer organisationalen Verankerung und Stabilität jener Strukturen und Prozesse, die diese Strategien stützen und nachhaltig fördern.

Aus methodologischer Sicht ist hervorzuheben, dass der Forschungsprozess selbst zu Veränderungen in den untersuchten Unternehmen beigetragen hat. Insofern kann hier ein responsiver Forschungsprozess konstatiert werden. Das individuelle Bewusstsein über das Lernen im Prozess der Arbeit und die zunächst implizite Unterscheidung von informellem und formellem Lernen wurden im Verlauf der Befragung explizit und es erfolgte - sowohl individuell als auch strukturell - eine veränderte Wahrnehmung des Lernens und der Lernprozesse.

Also ich würde mich freuen, wenn das Unternehmen schaffen könnte innerhalb des Arbeitslernbereiches leichte Verschiebungen vor zu nehmen. Im fachlichen Lernen mehr reinzugehen, das methodische Lernen deshalb nicht abzustellen, aber vielleicht in der Relation zu sehen, wie es tatsächlich notwendig ist. (07 RKH, S.24)

Die quantitative Auswertung der Skala Selbstreflexivität $(\mathrm{M}=2.85, \mathrm{SD}=$ .96) sowie die Skala strukturelle Reflexivität, $(\mathrm{M}=2.98$ und $\mathrm{SD}=.89)$ unterstützen, dass die Forschung als solche Reflexion auf individueller und struktureller Ebene auslöste. In dem Bewusstsein, dass organisationale Strukturen das Lernen im Prozess der Arbeit behindern können, äußerten die Beschäftigten den Bedarf, Rahmungen für informelles Lernen zu schaffen:

Und eigentlich wäre es schön wenn man das sehr viel mehr auch bei der Planung berücksichtigen könnte, dass man den Leuten dafür Zeit und Raum lässt sich zu entwickeln. (23AEA, S. 9)

\section{Literatur}

Baitsch, C. et al (1998): QUEM-report: Lernen im Chaos. Heft 52.

Bartels, R. (2001): Kompetenzentwicklung mitbestimmen. Besonderheiten der betrieblichen Mitgestaltung von Weiterbildung im Zusammenhang mit neuer Arbeitsorganisation für Betriebs- und Personalräte im Dienstleistungssektor. Darmstadt.

Davenport, T. H., Jarvenpaa, S. L., Beers, M. C. (1996): Improving Knowledge Work Processes. In: Sloan, P.: Management Review. o.O., S. 53-65. 
Dehnbostel, P. (2005): Lernen - Arbeiten - Kompetenzentwicklung: Zur wachsenden Bedeutung des Lernens und der reflexiven Handlungsfähigkeit im Prozess der Arbeit. In: Wiesner, G., Wolter A. (2005): Die lernende Gesellschaft - Lernkulturen und Kompetenzentwicklung in der Wissensgesellschaft. München. S. 111126.

Dehnbostel, P. (2007): Lernen im Prozess der Arbeit. Münster.

Dehnbostel, P, Molzberger, G., Overwien, B. (2003): Informelles Lernen in modernen Arbeitsprozessen - dargestellt am Beispiel von Kleinunternehmen der ITBranche. Berlin.

Frei, F. (1985): Kompetenzentwicklung in der Arbeit. In: Hoff, E., Lappe, L., Lempert W. (Hrsg.): Arbeitsbiographie und Persönlichkeitsentwicklung. Bern. S. 8898.

Heyse, V., Erpenbeck, J.,. Michel, L. (2002) QUEM-report: Lernkulturen der Zukunft, Heft 74.

Kelle, U. (1994): Empirisch begründete Theoriebildung - Zur Logik und Methodologie interpretativer Sozialforschung. Weinheim.

Lash, S. (1996): Reflexivität und ihre Doppelungen: Struktur, Ästhetik und Gemeinschaft. In: Beck, U., Giddens, A., Lash, S.: Reflexive Modernisierung. Eine Kontroverse. Frankfurt am Main. S. 195-286.

Mayring, P. (2002): Einführung in die qualitative Sozialforschung. Weinheim und Basel.

Mayring, P. (2008): Qualitative Inhaltsanalyse. Grundlagen und Techniken. Weinheim und Basel.

Meyer, R. (2006): Theorieentwicklung und Praxisgestaltung in der beruflichen Bildung - Berufsbildungsforschung am Beispiel des IT-Weiterbildungssystems. Bielefeld.

Meyer, R. (2011): Kompetenz- und Organisationsentwicklung im Kontext moderner Beruflichkeit - Theoretische und methodologische Annäherungen an die Sichtbarmachung organisationaler Deutungsmuster und pädagogischer Ordnungen. In: Niedermair, G. u.a.: Kompetenzen entwickeln, messen und bewerten, Sammelband 6 der Schriftenreihe für bbp, Linz. S. 43-57. (im Erscheinen).

Molzberger, G. (2007): Rahmungen informellen Lernens. Zur Erschließung neuer Lern- und Weiterbildungsperspektiven. Wiesbaden.

North, K., Güldenberg, S. (2008): Produktive Wissensarbeit(er). Wiesbaden.

Pfiffner, M., Stadelmann, P. (1999): Wissen wirksam machen. Wie Kopfarbeiter produktiv werden. Stuttgart.

Reichertz, J. (2003): Die Abduktion in der qualitativen Sozialforschung. Opladen.

Rohs, M. (2007): Zur Theorie formellen und informellen Lernens in der ITWeiterbildung. Hamburg.

Salmann, Y. (2009): Bildungseffekte durch Lernen im Arbeitsprozess. Verzahnung von Lern- und Arbeitsprozessen zwischen ökonomischer Verwertbarkeit und individueller Entfaltung am Beispiel des IT-Weiterbildungssystems. Bielefeld.

Staudt, E. (2002): Kompetenzentwicklung und Innovation. Die Rolle der Kompetenz bei Organisations-, Unternehmens- und Regionalentwicklung. (Edition QUEM, $14)$. 
Voigt, M., Weißbach, H.- J. (2005): Kompetenzentwicklung von Start-ups: Herausforderungen und Strategien. In: Arbeitsgemeinschaft Betriebliche Weiterbildungsforschung e.V. (Hrsg.): QUEM-report, ABWF. Heft 93: Kompetenzentwicklung in Start-up-Unternehmen. Berlin. S. 9-116.

Wilkens, U. (2004): Management von Arbeitskraftunternehmern. Psychologische Vertragsbeziehungen und Perspektiven für die Arbeitskräftepolitik in wissensintensiven Organisationen. Chemnitz.

Willke, H. (1998): Systemisches Wissensmanagement. Stuttgart 


\title{
Was kennzeichnet Kaufleute? -Ihr berufliches Denken und Handeln aus historischer, soziologischer und ordnungspolitischer Perspektive ${ }^{1}$
}

\author{
Franz Kaiser
}

\section{Aktuelle Diskurse und Anlässe}

Betrachten wir die aktuellen Diskurse zur kaufmännischen Berufsbildung, so sind sie einerseits von allgemeingesellschaftlichen Diskursen geprägt, die nach der Zulässigkeit und den Folgen kaufmännischen Denkens und Handelns fragen, wie dies insbesondere im Kontext der Finanzkrise geschieht, sich aber auch allgemeiner in der Literatur wiederfindet. So macht der 2009 erschienene Roman "Limit" von Frank Schätzing die bedrohlichen Folgen liberalisierter Märkte an einer fiktiven Energieressourcenerschließung auf dem Mond deutlich: „Alle werden den Preis bezahlen. Dieser letzte Satz drückte aus, was der Rückzug der Regierenden von den Prozessen der Globalisierung, die Verselbstständigung der Wirtschaft, die Privatisierung der Politik hinterlassen hatte: Ein Vakuum, das sich mit Kaufleuten füllte. Es definiert die Zukunft als Handelsware. “(ebd. S. 189)

Ein weiterer Diskurs findet sich bezogen auf die Veränderungen in der Arbeits- und Berufswelt. Er bezieht sich auf die organisatorischen und technologischen Veränderungen in den Unternehmen, die flexibilisierte Disposition der Ware Arbeitskraft (Boes/Kämpf 2008), die einhergehen mit einem „marktzentrierten Kontrollmodus“ (Kotthoff 1997, S. 293) und mit Begriffen wie „Kunden-“ und „Dienstleistungsorientierung“.

Eine dritte Diskurslinie bezieht sich auf die Reaktion der Ordnungspolitik auf den wirtschaftlichen Strukturwandel. Es ist eine Beschleunigung der Novellierung bestehender Aus- und Fortbildungsberufe und der Entwicklung neuer Ausbildungsberufe zu verzeichnen, die sich zumeist auf einzelne Dienstleistungsbranchen beziehen. ${ }^{2}$ Dabei wird einerseits argumentiert, dass angesichts des demografischen Wandels und der langfristigen Verwertbarkeit von Qualifikationen die Zahl der Berufe reduziert und die Profile breiter aufgestellt werden müssen. Andererseits wird von der Notwendigkeit der An-

1 Datengrundlagen für den Beitrag ist ein Forschungsprojekt an dem, neben dem Autoren auch Rainer Brötz, Benedikt Peppinghaus, Christin Brings und Silvia Annen am Bundesinstitut für Berufsbildung beteiligt sind.

2 Verwiesen sei beispielhaft auf die Personaldienstleistungskaufleute (2008), Sport- und Fitnesskaufleute (2007) und Veranstaltungskaufleute (2001). 
passung der kaufmännischen Ausbildungsberufszahl und mit ihr einhergehenden Differenzierung an die quantitative Entwicklung der neuabgeschlossenen Ausbildungsverträge gesprochen, bei denen ca. 60\% den kaufmännischen Berufen zugeordnet werden kann (BIBB 2010).

Der nachfolgende Beitrag bezieht sich insbesondere auf die beiden letztgenannten Diskurse, stützt sich hierbei auf aktuelle soziologische, historische Literaturstudien und Inhaltsanalysen der aktuellen Aus- und Fortbildungsordnungen und versucht auszumachen, was das Denken und Handeln qualifizierter Kaufleute, auf der Ebene der abhängigen Beschäftigung kennzeichnet.

\section{Beruf und seine Parameter}

Bei der Entwicklung von neuen Aus- und Fortbildungsberufen, wie auch bei der Klassifikation von Berufen (Bundesagentur 2011) stehen die Tätigkeiten, die in unterschiedlichen Einsatzgebieten in Erwerbsberufen ausgeübt werden im Mittelpunkt der Betrachtung (s. Abb.1). Von ihnen ausgehend werden bei der Entwicklung der Verordnungen die anzueignenden Kompetenzen bestimmt, die in den betrieblichen und schulischen Curricula und den Prüfungsbestimmungen festgelegt werden. Dabei werden die jeweilig eingesetzten technischen Hilfsmittel berücksichtigt und die Unterschiede, die sich aus der hierarchischen Stellung im Unternehmen (zumeist zwischen Aus- und Fortbildungsebene differenzierend) hinsichtlich der Tätigkeitsanforderungen ergeben.

Zugleich beeinflussen aber auch die Qualifizierungswege, die zu einer beruflichen Tätigkeit führen und die damit verbundene Unterschiedlichkeit der Wahrnehmung von Welt und der Lernkontext das Denken und Handeln und das Ansehen der Tätigkeit und deren tarifliche Eingruppierung. Der gesellschaftliche Stand eines Berufes wird somit zu einem weiteren Faktor für die berufliche Identität. Schließlich beeinflussen ökonomische, rechtliche, politische und kulturelle Rahmenbedingungen noch den Beruf, weil es durchaus einen Unterschied macht, diesen Beruf im Senegal oder in Deutschland auszuüben. 
Abbildung 1: Parameter der Berufsforschung

口

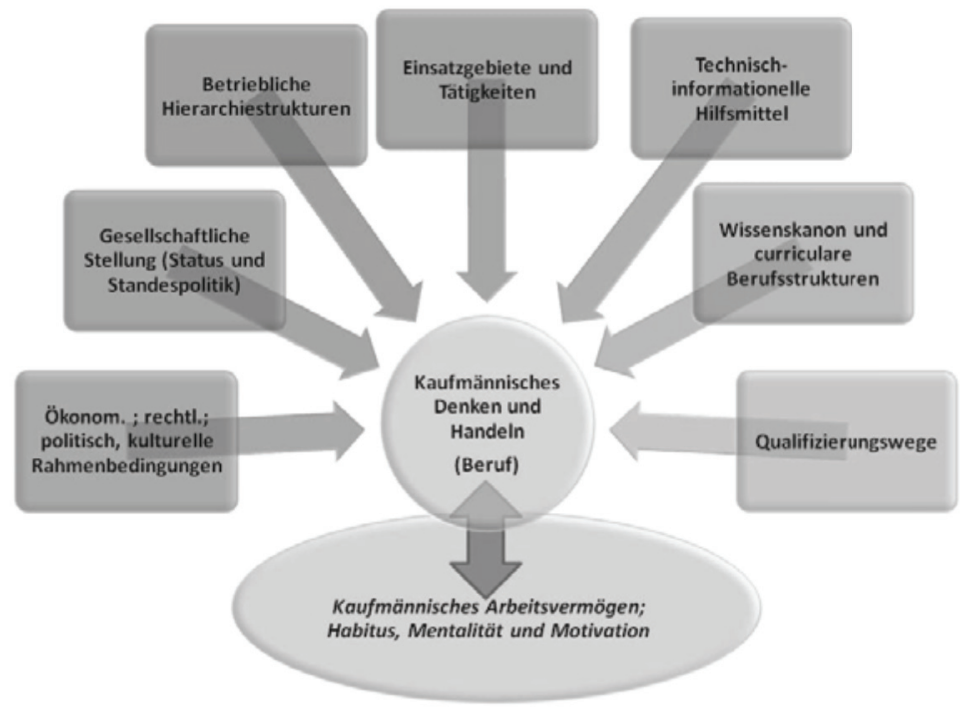

Nur wenn man all diese Faktoren mit berücksichtigt, wird Beruf mit seinen komplexen Vermittlungsfunktionen und dem Wechselverhältnis zwischen Bildungs-, Wirtschaftssystem und Persönlichkeit sichtbar (Beck/ Brater 1977, Deutschmann 2005, Schapfel-Kaiser 2008, Neuhaus 2011).

\section{Anmerkungen aus historischer und soziologischer Perspektive}

Die Börse tanzte ... um das goldene Kalb, und die Unternehmungssucht feierte wilde Orgien. Ein scheinbarer Erfolg war vorhanden und wie das Licht die Motten anzieht, so zog der missverstandene kaufmännische Beruf eine Menge ungeeigneter Leute, alte und junge, an, die ihm nicht zur Ehre gereichten. Freilich wurde bei diesem Taumel das erste vergessen, was den Kaufmann ausmacht: die Ausbildung. Lehrlinge wurden jugendliche Arbeiter. (Verband Deutscher Handlungsgehülfen 1906, S. 6, zitiert bei Reinsch 2011, S.204)

Die zuvor erwähnten Parameter von Berufen unterliegen einer gesellschaftlichen und historischen Entwicklung, in deren Verlauf sie sich verändern und 
epochenspezifisch in Erscheinung treten. In Anlehnung an Stratmanns (1993) acht „soziale Gefüge“ hat Reinisch (2011) die Entwicklung der kaufmännischen Berufe analysiert. Dabei wird deutlich, dass die „Urform“ der Kaufleute bei den Märkte versorgenden, umherziehenden Warenkaufleuten zu finden ist. Erst im 12 Jhdt. etablieren sich in Deutschland sesshafte Handelsherrn, die, mittels kleiner Kontore und Leitern der Faktoreien am jeweiligen Ort, in die Lage versetzt werden ein bedeutsames Kapital zu erwirtschaften und damit auch Einfluss auf die Politik an kirchlichen und weltlichen Höfen erlangen. Die nun einsetzende Standespolitik befördert die Anerkennung kaufmännischer Tätigkeit, die zuvor am gesellschaftlichen Rand angesiedelt und vergleichbar mit dem Ansehen fliegender Händler auf unseren heutigen Märkten betrachtet werden kann.

Innerbetrieblich und funktionsspezifisch treten erste Differenzierungen hinsichtlich Schreibarbeiten, Buchführung, Finanzierung und Transport auf, die sich aber nicht in Qualifikationsprofilen niederschlagen (ebd. S. 40). Lange Zeit entwickelt die kaufmännische Ausbildung, über die nur wenige Quellen vorhanden sind, verglichen mit der Lehrlingsausbildung der Zünfte, keinen systematisierten und institutionalisierten Charakter. Erst spät äußern die Kaufleute Kritik an den schulischen Bedingungen, fordern die Auseinandersetzung mit dem Rechnungswesen, der Sprache, den Sitten, den Umgang mit Waren und Transportwegen und machen sich für die Entstehung eigener Institutionen für die Qualifizierung ihres Nachwuchses stark. Da die Zahl der abhängig Beschäftigten unter den Kaufleuten über lange Zeit gering bleibt, ist die Figur des selbstständigen Kaufmanns lange Zeit prägend und daher entstehen auch keine Zusammenschlüsse der „,kaufmännischen Angestelltenschaft".

Dies ändert sich erst im ausgehenden 19. Jahrhundert. Kaufleute beginnen, im Unterschied zur bereits zuvor praktizierten Verlagsproduktion nun unmittelbar in die Herstellung von Waren zu investieren, werden Fabrikanten und Industrieunternehmer. Die Stellen für Angestellte in Bergbau, Baugewerbe, Handwerk und Industrie versieben fachen sich zwischen 1882 und 1907 von 99.076 auf 686.007 (ebd. S. 189). Die gemeinsame Chiffre der Selbständigkeit löst sich auf in Kaufleute als Unternehmer und kaufmännische Angestellte als Massenphänomen. Parallel dazu entwickelte sich der Handel in den Städten und brachte einen ähnlich expandierenden Arbeitsmarkt der Handelsgehilfen mit sich, der sich aus den männlichen Erwerbstätigen nicht mehr sättigen konnte und in beiden Zweigen zum breiten Einstiegstor für die weibliche Erwerbstätigkeit wurde. Ein Vorgang, der bei den sich nun gründenden Verbänden der kaufmännischen Angestellten nicht begrüßt wurde. ${ }^{3}$

3 In unterhaltsamer Weise nachzulesen bei Kaspar Schnetzlers Roman „Kaufmann und das Klavierfräulein“"von 2010, der anlässlich des 150-jährigen Bestehens des KV-Schweiz entstanden ist (vgl. KVZ 2011 aber auch Reinisch 2011 S. 195ff.). 
Versucht man nun Kennzeichnungen der Mentalität der Kaufleute anhand der historischen Erkenntnisse auszumachen, so lassen sich folgende Punkte festmachen:

1. Mit der Zunahme der Anerkennung verändert sich die Mentalität der Kaufleute, sie werden zu Gewinnern der sozialen Veränderungen des ausgehenden Mittelalters und zu geschätzten Wissensträgern und Beratern aber auch Finanziers.

2. Als selbstständige Handelsherren vermeiden sie Risiken und trachten nach Erhalt und behutsamen Ausbau ihrer ökonomischen Grundlagen und Geschäftsbeziehungen.

3. In abhängiger Beschäftigung sind sie vielfach ebenso Träger von Wissen und verfügen über relativ viel kulturelles Kapital in Form von Schrift, Mathematik und Fremdsprachen. Zugleich kennzeichnet ihr Qualifikationserwerb durch das Lernen in der Arbeit.

4. Mit dem sprunghaften, zahlenmäßigen Zuwachs in der kaufmännischen Angestelltenschaft verstärkt sich die Spaltung zwischen einfacher, schlecht vorgebildeter Angestelltenschaft (vor allem im Einzelhandel), die sich in gewerkschaftsähnlichen Verbänden organisiert und jenen, die sich in gehobenen Positionen befinden und deutlich stärker mit den Eigentümern identifizierten und in „Beamtenvereinen“ zusammenfinden ${ }^{4}$.

Verbunden mit dem Auftreten der kaufmännischen Angestelltenschaft als Massenphänomen entstehen auch erste Schriften der Soziologie nach der Jahrhundertwende, die sich mit dem Phänomen befassen (Lederer 1912). Sie machen das Zugehörigkeitsgefühl zur Mittelschicht als Unterscheidungsmerkmal aus, stellen eine starke Anfälligkeit für faschistische Ideologien und zunehmende Organisationsgrade der Angestelltenschaft fest. Bei den weiteren Studien bis hinein in die 1980er-Jahre wird die unterschiedliche Bewusstseinslage zwischen Arbeiter- und Angestelltenschaft wesentliches Thema. Konstatiert wird eine Erfolgs- und Karriereorientierung, die sich auch als inhaltliches Interesse und Beitragsorientierung kennzeichnen lässt (WeberMenges 2004). Zugleich wird deutlich, dass sich ein einheitlicher Begriff der

4 So beschreibt Reinisch: „Allerdings zeigte sich die überwiegende Zahl der Privatbeamten und Angestellten in der Industrie gar nicht an einer gewerkschaftlichen Organisation interessiert. Sie verstanden sich als Stand, der sich durch drei Aspekte vor den „niederen“ Angestellten im Warenhandel und den Verwaltungen auszeichnet und von diesen abgrenzt: durch erstens das ,innige“ Verhältnis zu den Angestellten- bzw. Eigentümerunternehmen und die besonders vertrauensvolle Weise der Zusammenarbeitet mit diesen, zweitens durch den ,gehobenen“ gesellschaftlichen und kulturellen Status, der sie trotz aller ökonomischen Unterschiede gegenüber den Unternehmern in der gesellschaftlichen Hierarchie fast auf die gleiche Stufe wie diese stellen würde, und drittens durch die gleiche oder nahezu gleiche soziale Herkunft aus dem gehobenen Bürgertum." (2011, S.201) 
Angestelltenschaft als analytische Kategorie nicht aufrechterhalten lässt. Hatte bereits Croner 1962 auf vier unterschiedliche Funktionen der Angestelltenschaft (arbeitsleitende, konstruktiv-gestaltende, analysierend -verwaltende und merkantile) hingewiesen (Haipeter 2011, S. 41) und damit deutlich gemacht, dass die Angestellten Aufgaben übernehmen, die ursprünglich die des Unternehmers gewesen sind. Zugleich machen Funktionsunterschiede auch die Unterschiede innerhalb der Angestelltenschaft deutlich. Nachfolgende Studien im Kontext des technischen Wandels und der Einführung von Lochkarten und Datenverarbeitungssystemen beobachteten Differenzierungen innerhalb der kaufmännischen Angestelltenschaft hinsichtlich ihrer Tätigkeitsniveaus (ebd. S. 55f.). In diesem Zusammenhang wird der Analysebegriff des Angestellten in der Industriesoziologie aufgegeben und einzelne Phänomene und Trends, die deren Arbeit betreffen werden zum Gegenstand der Forschung.

Zentrale und andauernde Trends sind:

1. Die zunehmende Bedeutung der Informatisierung der Arbeitsprozesse, mit der lange Zeit die Dequalifizierungsthese einherging und mit der heute, im Hinblick auf internationale Arbeitsteilung und Zusammenarbeit neue Qualifikationsanforderungen und Beschäftigungsrisiken verbunden sind (Boes/Kämpf 2010).

2. Die Subjektivierung und Entgrenzung von Arbeit, die mit der Flexibilisierung von Arbeitszeit und -ort einhergeht und insbesondere bei der projektförmigen Arbeitsorganisation und der Filialisierung in manchen Branchensektoren für kaufmännische Angestellte in sog. ,Scheinselbstständigkeit" und teilweise prekäre Beschäftigungssituationen mündet (Voss-Dahm 2009).

3. Die Ausweitung von Emotionsarbeit im Bereich der Dienstleistungen, die sich zum einen auf die Bewältigung der Widersprüche zwischen Unternehmens- und Kundeninteressen bezieht, aber auch auf die gestiegenen Anforderungen hinsichtlich Dienstleistungsqualität.

4. Die veränderten Steuerungsmechanismen innerhalb der Unternehmen, die sich als „marktzentrierter Kontrollmodus“ (Marrs 2010) bezeichnen lassen und die Rationalisierung von Arbeitsprozessen und die mentale Ausrichtung aller Beschäftigten auf die Steigerung des Unternehmenswerts an der Börse (Finanzialisierung) einfordern und Zielerreichung zur Legitimationsgrundlage für die Unternehmenszugehörigkeit erklärt. Damit einhergehen die Zentralisierung von strategischen Entscheidungen und die operative Dezentralisierung, die über Ziele gesteuert wird (Haipeter 2011, S. 135). 
Zusammenfassend bedeutet dies für die Kaufleute in den Unternehmen, dass der Umgang mit IT-Technologie und die Übersetzung von Logiken und Symbolen in andere Kontexte und Sprachen zunehmend zum Alltag ihres beruflichen Handelns gehört. ${ }^{5}$ Zugleich handeln sie in zunehmend offenen Entscheidungssituationen, deren emotiononale Komponenten insbesondere an den Kommunikationsschnittstellen innerhalb und außerhalb der Unternehmung von ihnen gestaltet werden. Die Widersprüche, die sich aus den Interessen von Unternehmenszielen, Zielen der Beschäftigten, der Kundinnen und Kunden sowie ggf. aus gesellschaftlichen Interessen ergeben müssen von ihnen wahrgenommen, ausgehalten oder ggf. aufgelöst und in akzeptable Kompromisse überführt werden. Dies setzt die Reflexion der Zusammenhänge, die Kenntnis der Geschäftsprozesse und kreative Gestaltungskompetenz (Vgl. Kapitel 3.4 in Brater/Freygarten/Rahmann/ Rainer 2011) zunehmend voraus und lässt die Befassung mit Fragen unternehmerischer Selbstständigkeit $\mathrm{zu}$ einem Bestandteil von Ausbildung werden (PauliniSchlottau 2005).

Bei all diesen Erkenntnissen sind Fragen nach dem Kern der Fachlichkeit kaufmännischen Denkens und Handelns noch nicht beantwortet.

\section{Ordnungsmittelanalyse als Erkenntnisquelle ${ }^{6}$}

Um die Gemeinsamkeiten und Unterschiede der kaufmännischen Berufe genauer zu betrachten, wurde in den vergangenen zwei Jahren am Bundesinstitut für Berufsbildung eine systematische Analyse der Verordnungen und Rahmenlehrpläne für kaufmännisch-betriebswirtschaftliche Aus- und Fortbildungsberufe durchgeführt (Brötz, Schapfel-Kaiser 2010). Diese Analyse umfasst alle dual ausgebildeten Berufe kaufmännischer Prägung sowie die bundesweit verordneten, kaufmännischen Fortbildungsberufe. Die Ordnungsmittel werden als Quelle für gegenwärtige kaufmännische Tätigkeiten und Qualifikationen betrachtet. Eine Verifizierung der Analysen erfolgt in 2012 anhand einer repräsentativen Befragung tätiger Kaufleute im Nachgang zur BIBB-BAuA Erwerbstätigenbefragung (Hall u.a. 2011).

Bei dieser Analyse wurde kein theoretisches Modell kaufmännischer Qualifikation zugrunde gelegt, sondern vielmehr aus den vorhandenen Ver-

5 Der Umgang mit den Abbildsystemen die sich in den innerbetrieblichen Steuerungsystemen (ERP) finden und zu einer anderen Qualität des Umgangs mit dem betrieblichen Rechnungswesen geführt haben (Preiss 2005), ist in diesen Punkt eingeschlossen.

6 Es können an dieser Stelle nur grob die Vorgehensweise und skizzenhafte Ergebnisse wiedergegeben werden. Ausführlicher wird darüber berichtet in Brötz. u.a. 2011.

7 Die Berufsauswahl deckt sich weitgehend mit den, von Bank (2010, S.593ff.) gelisteten Berufen für die berufliche Fachrichtung Wirtschaft und Verwaltung. 
ordnungstexten heraus Kategorien und Codierungen entwickelt mit deren Hilfe die Texte analysiert wurden. Durch den Einsatz von mehreren Codiererinnen und Codierern war eine genaue Beschreibung der angewendeten Codes und deren Abgrenzung notwendig sowie die Berechnung der Intercoder-Reliabilität, um zu prüfen, ob das Analyseschema weitgehend einheitlich angewendet wurde (vgl. Brötz u.a. 2011, S. 49ff., Müller-Benedict 1998). Um sich den Gesamtumfang dieser Forschungsarbeit zu verdeutlichen, seien ein paar Zahlen angefügt: Insgesamt wurden 55 Ausbildungs- und 33 Fortbildungsberufe untersucht. Der Codebaum umfasste über 80 Codes und Subcodes und es wurden ca. 42.000 Codierungen vorgenommen. Auf der obersten Ebene wurden 4 inhaltsbezogene Kategorien unterschieden:
A) Kaufmännische Gemeinsamkeiten
B) Kaufmännische Besonderheiten und Vertiefungen
C) Übergreifende Qualifikationen
D) Nicht kaufmännische Qualifikationen

Konzentrieren wir uns zunächst auf den Bereich der Gemeinsamkeiten, so wird deutlich, dass er bezogen auf alle kaufmännischen Ausbildungsberufe einen Anteil von ca. 70\% aufweist (s. Abb. 2). Dabei weisen die Bereiche „Kaufmännische Steuerung und Kontrolle“, „Absatzwirtschaft“ (die Marketing, Verkauf und Beratung einschließt) und "Information und Kommunikation" die größten Anteile auf. 
Abbildung 2: Häufigkeitsverteilung inhaltsbezogene Kategorien, Bereich der kaufmännischen Gemeinsamkeiten (1-9) untergliedert

口

\section{Die Häufigkeitsverteilung in den Ausbildungsordnungen der inhaltsbezogenen Kategorien} ( $n=22957$ )

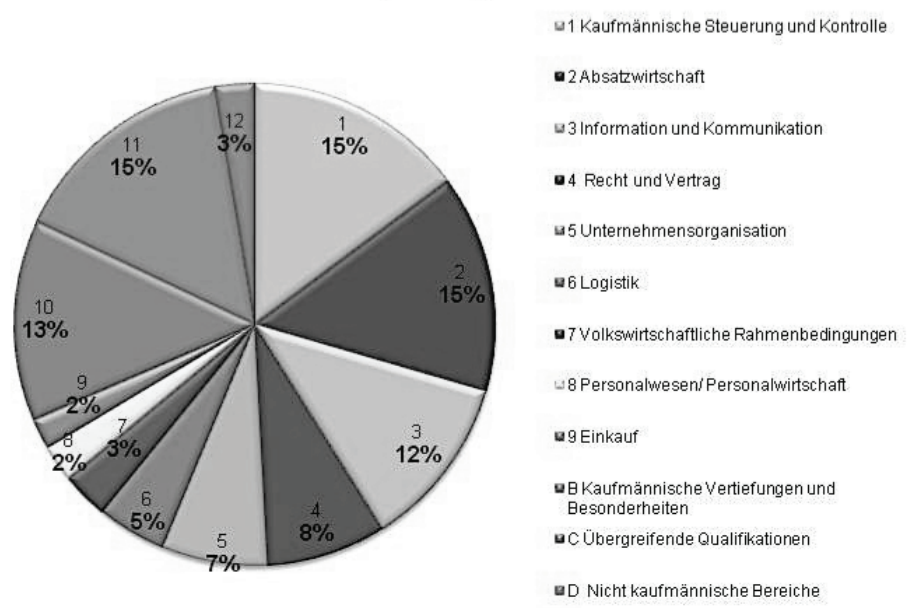

Betrachtet man allerdings die Ergebnisse im einzelberuflichen Vergleich, so werden deutliche Unterschiede in den Spektralanalysen erkennbar (Abb. 3). Findet sich bspw. bei den Waren- und Finanzkaufleuten ein Anteil von über $30 \%$ im Bereich der Absatzwirtschaft, so ist dieser deutlich geringer bei den Verwaltungs- und Logistikberufen. Bei letztgenannten steigt dafür der Bereich Logistik drastisch an. Noch deutlicher werden die Unterschiede, wenn man den Bereich der kaufmännischen Vertiefungen und Besonderheiten betrachtet. Hier finden sich Codes wie „Warenwirtschaftssystem“ oder auch spezielle Rechtsgebiete, die dann noch stärker die Warenkaufleute, bzw. die Rechtsberufe unterscheiden. Dennoch finden sich auch nach der Kontrastierung des Codebaums an drei gewerblich-technischen Berufen und der daran anschließenden Herausnahme von übergreifenden Qualifikationen aus dem Bereich der Gemeinsamkeiten, eine hohe Parallelität der „Kerne“ kaufmännischer Qualifikationen.

Die Ordnungsanalyse gibt so nicht nur hilfreiche Hinweise für die Möglichkeit der Gruppenbildung innerhalb der kaufmännischen Berufe, sondern auch Hinweise auf gemeinsame Handlungsfelder, die auch für Empfehlungen für die künftige Gestaltung kaufmännischer Fortbildungsordnungen genutzt werden können (Kaiser/Brötz 2011). 
Abbildung 3: Prozentuale Häufigkeitsverteilung der kaufmännischen Gemeinsamkeiten auf ausgewählte Ausbildungsberufe

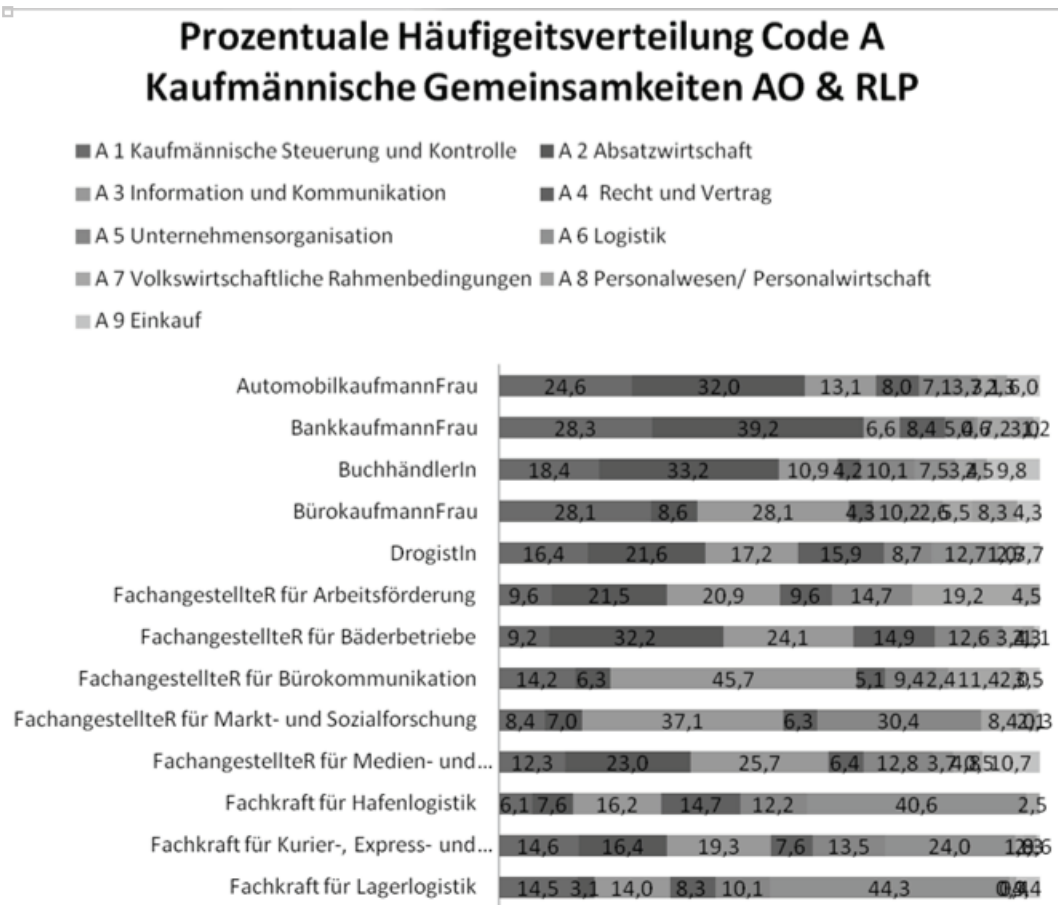

\section{Zur Bildung eines Begriffs des Kaufmännischen Denkens und Handelns}

Verdichten wir die Erkenntnisse aus diesen drei Betrachtungen zu einer Theorie des kaufmännischen Denkens und Handelns, so lassen sich mehrere Ebenen unterscheiden. In den konkreten Handlungsvollzügen auf der Ebene ihres unmittelbaren Tätigkeitssystems kennzeichnet die Kaufleute neben kommunikativen Handlungen in zwischenmenschlicher Interaktion, das Umgehen mit planenden, steuernden, Verbindlichkeit erzeugenden, dokumentierenden und bilanzierenden Abbildsystemen, die sich auf Referenzhandlungsfelder beziehen (Tramm 2009). Dabei berücksichtigen sie in besonderer Weise ihre innerbetrieblichen Rahmenbedingungen, Zielvorgaben und Schnittstellen der Geschäftsprozesse und die Außenbeziehungen des Un- 
ternehmens $\mathrm{zu}$ externen Märkten, Rechtsvorschriften, gesellschaftspolitischen Trends. Die damit verbundenen Handlungsentscheidungen und -vollzüge, die sich primär als Mittlerfunktionen beschreiben lassen, erfolgen überwiegend unter der Ägide des ökonomischen Prinzips, dass der unternehmerischen Logik entspringt, die mit der Verlagerung der unternehmerischen Aufgaben auf die kaufmännischen Angestellten, auch mittels der wirtschaftsberuflichen Erziehung von diesen internalisiert wurde. Damit werden kaufmännische Angestellte zu Agentinnen und Agenten betriebswirtschaftlicher Verwertungslogik zu deren Verbreitung auch der massive Aufwuchs der kaufmännischen Angestelltenschaft und deren Qualifikation beigetragen haben kann. Wirtschaftspädagogische Selbstreflexion über die Qualifizierungsziele der kaufmännischen Berufsbildung und ihrer gemeinwohlorientierten Aufgabe ist permanent zur Verantwortung ihrer Ziele aufgefordert (Lempert 2006). Diese, von Lempert, in Anlehnung an Zabeck (2004), an die Wissenschaft herangetragene Forderung gilt nicht minder für die berufsbildungspolitischen Gestaltungsakteuren im Kontext der Entwicklung von Aus- und Fortbildungsordnungen.

\section{Literatur}

Bank, V. (2010): Berufliche Fachrichtung Wirtschaft und Verwaltung. In: Pahl, J.P./Herkner, V. (Hrsg.): Handbuch Berufliche Fachrichtungen. Bielefeld

Beck, U./Brater, M. (1977): Die soziale Konstitution der Berufe: Materialien zu einer subjektbezogenen Theorie der Berufe. 2Bde. Frankfurt a.M

BIBB (Hrsg.) (2010): Datenreport zum Berufsbildungsbericht, Bonn

Boes, A./Kämpf, T. (2010): Zeitenwende im Büro: Angestelltenarbeit im Sog der Globalisierung. In: WSI Mitteilungen, H. 12, $63 \mathrm{Jg}$.

Brater, M./Freygarten, S./Rahmann, E./Rainer, M. (2011): Kunst als Handeln - Handeln als Kunst. Was die Arbeitswelt und Berufsbildung von Künstlern lernen können. Bielefeld

Brötz, R./Schapfel-Kaiser, F. (Hrsg.) (2009): Anforderungen an kaufmännischbetriebswirtschaftliche Berufe aus berufspädagogischer und soziologischer Sicht Bonn

Brötz, R./Schapfel-Kaiser, F. (2010): Gemeinsamkeiten in kaufmännischen Ausbildungsberufen ermitteln. Zwischenergebnisse einer computergestützten Dokumentenanalyse. In BWP, H. 4, 39. Jg.

Brötz, R./ Kaiser, F./Brings, C./Peppinghaus, B./Warmbold-Jaquinet, V./Krieger, A./Noack, I./Nies, N./Schaal, T. (2011): Gemeinsamkeiten und Unterschiede kaufmännisch-betriebswirtschaftlicher Aus- und Fortbildungsberufe (GUK). Zwischenbericht. Bonn, (http://www2.bibb.de/tools/fodb/pdf/zw_42202.pdf) 
Bundesagentur für Arbeit (2011): Klassifikation der Berufe 2010, Bd.1, Nürnberg

Deutschmann, C. (2005): Latente Funktionen der Institution des Berufs. In: Jacob, M. /Kupka, P.: Perspektiven des Berufskonzepts - Die Bedeutung des Berufs für Ausbildung und Arbeitsmarkt. Beiträge für Arbeitsmarkt- und Berufsforschung Nr. 297, Nürnberg

Haipeter, T. (2011): Kaufleute zwischen Angestelltenstatus und Dienstleistungsarbeit - eine soziologische Spurensuche. Wissenschaftliches Diskussionspapier des BIBB, Nr. 126, Bonn

Hall, A. u.a. (2011): BIBB-BAuA-Erwerbstätigenbefragung 2011/2012. Antragstext. (http://www2.bibb.de/tools/fodb/pdf/at_21304.pdf)

Kaiser, F./Brötz, R. (2011): Konzept für berufsübergreifende Qualifikationen in kaufmännischen Fortbildungsregelungen. Abschlussbericht. (http://www2.bibb. de/tools/fodb/pdf/eb_42344.pdf)

Kaufmännischer Verband Zürich (2011): 150 Jahre Kaufmännischer Verband Zürich. Kämpfen, Lernen, Feiern. Zürich

Kotthoff, H. (1997): Führungskräfte im Wandel der Firmenkultur. Quasi-Unternehmer oder Arbeitgeber? Berlin

Lederer, E. (1912): Die Privatangestellten in der modernen Wirtschaftsentwicklung, Tübingen

Lempert, W. (2006): Wirtschaftsberufliche Erziehung angesichts des real expandierenden Kapitalismus. Ein Beitrag zur Funktionsbestimmung zeitgemäßer Berufs- und Wirtschaftspädagogik. In: Zeitschrift für Berufs- und Wirtschaftspädagogik, 102,1

Marrs, K. (2010): Herrschaft und Kontrolle in der Arbeit. In: Böhle, F./Pongratz, G.G./Wachtler, G. (Hrsg.): Handbuch Arbeitssoziologie,. Wiesbaden

Müller-Benedict, V (1998): Der Einsatz von Maßzahlen der Intercoder-Reliabilität in der Inhaltsanalyse. In: Zeitschrift für Soziologie der Erziehung und Sozialisation. 1, 105. (http://www.ssoar.info/ssoar/files/2008/339/intercod.pdf)

Neuhaus, L. (2011): Wie der Beruf das Denken formt. Berufliches Handeln und soziales Urteil in professionssoziologischer Perspektive. Marburg

Paulini-Schlottau, H. (2005): Unternehmerische Selbständigkeit in die Berufsbildung integrieren! Leitartikel. (http://www.bibb.de/de/17016.htm)

Preiss, P. (2005): Förderung kaufmännischer Kompetenzen mit Hilfe des wirtschaftsinstrumentellen Rechnungswesens - aus fachlich-curricularer Perspektive. In: Sembill, D./Seifried, J. (Hrsg.). Rechnungswesenunterricht am Scheideweg: Lehren, Lernen und Prüfen. Wiesbaden

Reinisch, H. (2011): Geschichte der kaufmännischen Berufe. Wissenschaftliches Diskussionspapier des BIBB, Nr. 125, Bonn

Schapfel-Kaiser, F.: Beruf und Zeit. Bonn

Schätzing, F. (2009): Limit. Roman, Köln

Stratmann, K. (1993): Die gewerbliche Lehrlingserziehung in Deutschland. Modernisierungsgeschichte der betrieblichen Berufsbildung. Band 1. Frankfurt am Main

Tramm, T. (2009): Berufliche Kompetenzentwicklung im Kontext kaufmännischer Arbeits- und Geschäftsprozesse. In: Brötz/Kaiser 2009. 
Voss-Dahm, D. (2009): Über die Stabilität sozialer Ungleichheit im Betrieb: Verkaufsarbeit im Einzelhandel. Berlin

Weber-Menges, S. (2004): Arbeiterklasse oder Arbeitnehmer? Vergleichende empirische Untersuchung zu Soziallage, Lebenschancen und Lebensstilen von Arbeitern und Angestellten in Industriebetrieben. Wiesbaden

Zabeck, J. (2004): Berufserziehung im Zeichen der Globalisierung und des Shareholder Value. Wirtschaftspädagogisches Forum Bd. 26. Paderborn 



\title{
Kompetenzentwicklung und organisationale Veränderung am Beispiel von Festivalveranstaltern
}

\author{
Petra F. Köster
}

\section{Problemstellung}

$\mathrm{Zu}$ der Interdependenz von individueller Kompetenzentwicklung und organisationaler Veränderung gibt es bisher kaum empirische Untersuchungen. Im aktuellen berufs- und betriebspädagogischen Diskurs bildet der Begriff der Kompetenz, als Ziel beruflicher Qualifizierungsprozesse, eine Basis der wissenschaftlichen Auseinandersetzung (vgl. exempl. Dehnbostel 2007; Arnold 2005; Bohlinger/Münk 2008; Bethscheider et al. 2007; Gnahs 2007). Prozesse der organisationalen Veränderung werden demgegenüber zwar thematisiert (vgl. Arnold 1996; Dietzen 2004), sind aber nicht zentral. ${ }^{1}$ Bei der Problematisierung der Interdependenz von Kompetenz- und Organisationsentwicklung handelt es sich im pädagogischen Fachdiskurs eher um normative Forderungen (vgl. Geißler 1996), während empirische Studien die dem Zusammenhang von Kompetenz- und Organisationsentwicklung nachgehen nur vereinzelt vorliegen (vgl. Bartels 2001; Hartz 2004). So ist zwar davon auszugehen, dass sich Kompetenzentwicklung und organisationale Veränderung wechselseitig Beeinflussen und aufeinander angewiesen sind, ob und wie dies geschieht wurde allerdings noch kaum empirisch erforscht. Ebenso wird davon ausgegangen, dass diese Interdependenz eine Voraussetzung für die Innovationsfähigkeit einer Organisation darstellt, doch auch zu dieser Annahme liegen noch kaum Studien vor.

Um Betriebe systematisch als pädagogische Handlungsräume zu gestalten bedarf es jedoch einer genauen Kenntnis der innerbetrieblichen Gegebenheiten. Dieser Beitrag zielt, mit Hilfe eines ethnographischen Forschungsdesigns, auf eine solche Exploration am Beispiel der Festivalbranche als innovationsintensive Betriebe. Dabei werden Betriebe als kommunikativ erzeugte Organisationen beschrieben und analysiert mit dem Ziel, gute Praxisbeispiele zu liefern und zu einer empirisch fundierten Modellbildung beizutragen. Im Mittelpunkt der Analyse steht die Frage, wie eine funk-

1 Innerhalb des betriebswirtschaftlichen Fachdiskurses wird unter dem Begriff „Organisationsentwicklung“ ein Bündel von Managementtechniken zur Analyse und systematischen Veränderung von Organisationen verstanden. Die hier vorgestellte Arbeit befasst sich allerdings mit Veränderungsprozessen in Organisationen im Allgemeinen, bezieht also auch nicht-intendierte Veränderungsprozesse ein. 
tionale Verknüpfung von Kompetenzentwicklung und organisationaler Veränderung in einer von Innovationsdruck geprägten, betrieblichen Praxis gestaltet sein kann.

\section{Forschungsleitende Annahmen}

Kompetenz- und Organisationsentwicklung sind - so die erste Annahme voneinander abhängige, interdependente Prozesse, welche grundlegend sind für die Konkurrenzfähigkeit innovationsintensiver Unternehmen auf der einen Seite, und die Entwicklungsmöglichkeiten der Beschäftigten auf der anderen Seite. Innovationsintensive, und somit wissensbasiert arbeitende Branchen und Betriebe sind dadurch gekennzeichnet, dass sie auf eine permanente Aktualisierung von Wissen und die aktive, kreative Nutzung desselben angewiesen sind um immer Neues, also Innovatives, hervorbringen $\mathrm{zu}$ können (vgl. exempl. Staudt 2002; Anand/Gardner/Morris 2007; Wilkens 2004). Befassen wir uns mit wissensintensiven Branchen und Berufen, ist davon auszugehen, dass eine permanente Kompetenzentwicklung im Sinne des lebenslangen Lernens eine wesentliche Voraussetzung darstellt, die sich auch auf die Entwicklungsmöglichkeiten der Organisationen auswirkt. Umgekehrt provozieren organisationale Veränderungen Anpassungsleistungen ihrer Mitglieder, die als Lernprozesse beschrieben werden können.

Die Verknüpfung von organisationalen und individuellen Entwicklungsprozessen erfolgt über kommunikative Strukturen oder - genau genommen bestehen Organisation gemäß der luhmannschen Systemtheorie aus Kommunikation (vgl. exempl. Luhmann 1987, S. 66f.). Folglich müssen Betriebe, die dauerhaft erfolgreich sein wollen, Kommunikationsformen entwickeln, die individuelle und organisationale Entwicklungsprozesse aneinander anschließen. Darüber hinaus ist anzunehmen, dass es unterschiedliche kommunikative Lösungswege für diese Herausforderung gibt, dass verschiedene Organisationen also unterschiedlich, aber gleichermaßen erfolgreich kommunizieren und so Kompetenzentwicklung und organisationale Veränderung verknüpfen.

Den Hintergrund der Studie bildet die Annahme, dass es Kommunikation in innovativen Unternehmen gibt, die die Verknüpfung von Kompetenz- und Organisationsentwicklung befördert und so dazu führen, dass die Organisation innovationsfähig bleibt. Darüber hinaus ist davon auszugehen, dass nicht nur formalisierte Strukturen, sondern in erheblichem Maße auch 
informelle Kommunikation entscheidend für den Erfolg von Organisationen ist (vgl. Böhle und Bolte 2002). ${ }^{2}$

Als Beispiel für innovationsintensive Betriebe dienen in der hier vorgestellten Studie Veranstalter von Musikfestivals: Die permanente Aktualisierung von Wissen erfolgt dabei zum Beispiel im Bereich der Technik. Sowohl bei Licht, Ton oder Bühnenbau, müssen die Beschäftigten ihr Wissen und ihre Fähigkeiten parallel zum technischen Fortschritt weiterentwickeln um ihre berufliche Handlungskompetenz aufrecht zu erhalten. Viele der Berufe rund um die Organisation von Festivals wurden erst in den letzten Jahren (seit Beginn der 90er Jahre) entwickelt und als Ausbildungsberufe etabliert. Doch trotz dieser Formalisierungsbestrebungen erfolgen viele Aneignungsprozesse, gerade nach Abschluss der Ausbildung weiterhin autodidaktisch. Darüber hinaus ist es grundlegend für die Organisation von Events, dass sich die beteiligten Personen mit ihren hochspezialisierten Wissensbeständen und Fähigkeiten austauschen und aufeinander abstimmen. Aushandlungs- und Klärungsprozesse sind daher fester Bestandteil von Organisationen in der Festivalindustrie.

\section{Zielsetzung der Untersuchung}

Zielsetzung der Untersuchung ist folglich die explorative Analyse der oben beschriebenen (weitestgehend informellen) Kommunikationsprozesse als Voraussetzung für innovative Organisationen am Beispiel von Veranstaltern von Musikfestivals, da diese als exemplarisch für innovationsintensive Betriebe gelten können. Dabei wird die Frage nach erfolgreicher kommunikativer Verknüpfung von Kompetenzentwicklung und organisationaler Veränderung auf unterschiedlichen Analyseebenen bearbeitet: Den Ausgangspunkt bildet der berufs- und wirtschaftspädagogische Fachdiskurs, indem sich die Studie auf pädagogische Kompetenzbegriffe bezieht und Organisationen als potentielle, gestaltbare pädagogische Räume analysiert. Dabei wird durch den Einbezug von betriebswirtschaftlichen und soziologischen Theorien zur Organisationsentwicklung und organisationaler Veränderung ein interdisziplinärer Zugang gewählt.

Der methodische Zugang erfolgt über ethnografische Verfahren (Wolcott 2008; Geertz 1983), die bisher für die Berufs- und Wirtschaftspädagogik kaum genutzt wurden, aber eine vielversprechende Erweiterung etablierter Feldzugänge darstellen (Meyer/Elsholz 2009). Wie diese Methoden für die Berufs- und Wirtschaftspädagogik nutzbar sind, soll daher im Rahmen der

2 Zur empirischen Erforschung pädagogischer Gestaltbarkeit informeller Lernprozesse in Unternehmen siehe auch Gabriele Molzberger (2008): „Rahmungen informellen Lernens“. 
Studie eruiert werden. Darüber hinaus ist ein ethnographischer Zugang für die Erfassung kommunikativer Strukturen geeignet und fügt sich daher in den (kommunikations-)theoretischen Rahmen. Neben der Reflexion von methodischen und theoretischen Bearbeitungen pädagogischer Forschungsfragen leistet die Studie auch einen Beitrag zum Fachdiskurs sowie zur Praxisgestaltung: Durch die Analyse von ,good-practice-strategien“ zur Verknüpfung von Kompetenzentwicklung und Organisationsentwicklung wird ebenso die Grundlage zur Entwicklung von (theoretischen) Modellen gelegt, sowie zur empirisch fundierten, systematischen Gestaltung von Kommunikation als Voraussetzung für individuelle und organisationale Entwicklungsmöglichkeiten.

\section{Methodisches Vorgehen}

Ethnographie ist weniger eine Forschungsmethode, als „a way of seeing“ (Wolcott 2008). Der Eigenlogik des Feldes folgend, werden dabei alle verfügbaren Zugänge zum Feld genutzt, wie teilnehmende Beobachtungen, aber auch Dokumentenanalysen und narrative Interviews. Im Anschluss an eine insgesamt vierwöchige Feldphase in zwei verschiedenen Organisationen verfasse ich eine „dichte Beschreibung“ in Anlehnung an Clifford Geertz (1983). Diese beinhaltet (im Gegensatz zur „dünnen Beschreibung“) nicht nur die Schilderung dessen, was zu sehen und zu hören war, sondern auch die Bedeutung des Beobachteten in dem jeweiligen Kontext. Nach Geertz ist das Ziel von dichten Beschreibungen, dass diese die Grundlage für noch zutreffendere Beschreibungen liefern. Die theoretische Anbindung erfolgt nach Geertz ausschließlich über die Verwendung wissenschaftlich geprägter Begriffe (vgl. Geertz 1983, S.33). ${ }^{3}$ In der hier vorgestellten Studie werden begriffliche Bezüge zum berufspädagogischen Diskurs hergestellt, da kompetenzentwicklungsförderliche Aspekte im Fokus der Aufmerksamkeit stehen. Auf metatheoretischer Ebene kommen differenztheoretischen Analyserahmen (v.a. Luhmann 1994, 2009) zum Tragen. Auf der Grundlage der „dichten Beschreibung“ der kommunikativen Strukturen in den untersuchten Organisationen und deren Vergleich ist es das Ziel der Arbeit, Implikationen für Theorie und Praxis zu erarbeiten (s.o.).

3 Vor diesem Hintergrund ist die Kritik an der Ethnographie nachvollziehbar, sie sei „detailverliebt" (Vogd 2009, S. 54). Es ist Teil des eigenen Lernprozesses im Feld, des eigenen Vertraut-Werdens mit der Organisation die Relevanz von Details gemäß ihrer systeminternen Bedeutung immer besser beurteilen zu können. Ich stelle allerdings in Frage, ob Ethnographie bei den Details verbleiben muss, oder ob man diesen Aneignungsprozess nicht auch für das Herausarbeiten praxis- und theorierelevanter Erkenntnissen nutzen kann. 
Das Vorgehen im Rahmen der Studie ist abduktiv, verknüpft also induktive und deduktive Verfahren. Dadurch wird eine wechselseitige Bereicherung von Theorie und Praxis angestrebt (vgl. Bude 2008, Meyer und Elsholz 2009, Reichertz 2000). Die formulierten Annahmen sind also keineswegs Hypothesen, die es zu überprüfen gilt, sondern sie bilden eine Explikation der theoretischen Grundlagen, vor deren Hintergrund die Beobachtung stattfand. Dadurch wird der faktischen Unmöglichkeit einer voraussetzungslosen Forschung Rechnung getragen.

\section{Erste Ergebnisse}

\section{Merkmale der Festivalbranche}

Das Forschungsfeld wurde unter anderen auf der Basis der Vorannahme ausgewählt, dass Festivalveranstalter nicht nur wissensintensiv, sondern auch innovationsintensiv arbeiten (s.o.). Im Rahmen der empirischen Analyse konnte diese These bestätigt werden, sofern ein weiter Innovationsbegriff zugrunde gelegt wird, der die Anpassung organisationaler Strukturen an Trends und andere jeweils aktuelle Erfordernisse einschließt (vgl. exempl. Horsch 2003, S. 5; Staudt/Kriegesmann 2002, S. 29; vgl. Domsch et al 1995, S. 14). Festivals zu veranstalten ist nicht nur eine logistische Herausforderung, sondern Events stellen auch ein künstlerisches Angebot dar. Daher muss mit immer neuen Bands, aber auch innovativen Gestaltungsideen aufgewartet werden und eine Anpassung an aufkommende Trends stattfinden (wie zum Bespiel so genannte „Walking Acts“4 ${ }^{\text {“4 }}$ oder die Organisation von ElektroPartys nach den Konzerten), um genug Publikum anzuziehen. Ausgeprägte Problemlösungsfähigkeiten erfordern auch unvorhersehbare Ereignisse, wie zum Beispiel Stürme, gesetzliche Veränderungen oder Vandalismus.

Die Branche der Festivalveranstalter ist darüber hinaus geprägt durch ein seit Jahren anhaltendes Wachstum, größer werdenden Konkurrenzdruck und die Dominanz einzelner großer Unternehmen mit entsprechenden Handlungsspielräumen. Die Konkurrenz entsteht vor allem durch steigende Kosten. Diese steigen vor allem deshalb, weil die GEMA ab dem Jahr 2012 stufenweise ihre Tarife erhöht. Dies wiederum ist eine Folge der schwindenden wirtschaftlichen Bedeutung des Verkaufs von Tonträgern. Aus demselben Grund müssen sich Künstler vermehrt über Tourgagen finanzieren. Dadurch

4 Als „Walking Acts“ werden die Auftritte von Künstlern bezeichnet, die sich über das Festivalgelände bewegen und an verschiedenen Stellen und zu verschiedenen Zeitpunkten ihr Programm darbieten. Das können Musiker sein, aber auch Clowns, Jongleure oder riesige beleuchtete Puppen, die von mehreren Künstlern gesteuert durch das Publikum laufen. 
steigen auch die Kosten der Veranstalter für die Künstler. Sie müssen mit dem daraus entstehenden verschärften ökonomischen Erfolgsdruck umgehen und entwickeln dementsprechend Strategien, wie die Erhöhung von Eintrittspreisen.

Auch die Arbeit mit Freiwilligen spielt eine hervorzuhebende Rolle. Dabei teilt sich die Gruppe der Freiwilligen bei einigen Festivals auf in einen kleinen „harten Kern“, der über das ganze Jahr involviert ist, und eine große Zahl von Helfern über die Zeit des Festival (vergleichbar mit einer relativ kleinen „Kernbelegschaft“ gegenüber einer großen Randbelegschaft).

In Reaktion auf den sich verstärkenden Erfolgsdruck bilden die kleineren Festivals Netzwerke, die die Vorteile der großen Betriebe zum Teil kompensieren können. So sind mehrere Festivals im Zusammenschluss eher in der Lage internationale Bands anzuwerben, weil sie diese für mehrere, terminlich aufeinander abgestimmte Auftritte buchen können, ihnen also quasi kleine Touren durch Deutschland vorstrukturieren. Auch Kontakte zu Kooperationspartnern wie Booking- oder Bühnenbaufirmen und die Zahl der ehrenamtlichen Helfer können so potenziert werden.

Im Folgenden werden die beiden untersuchten Fälle kurz dargestellt und anschließend im Hinblick auf die Interdependenz von Kompetenz- und Organisationsentwicklung verglichen. Die Ziele der Organisationen und die organisationalen Strukturen bei den untersuchten Veranstaltern sind unterschiedlich, aber schließen gleichermaßen erfolgreich Kompetenzentwicklung an Organisationsentwicklung an.

\section{Fall A "die Idealisten"}

Fall A ist ein so genanntes „Umsonst und Draußen-Festival“ (U\&DFestivals) und fand 2011 zum 33. Mal statt. Die ersten Male war der Grund für die Veranstaltung, Geld für ein autonomes Jugendzentrum zu erwirtschaften. Es handelt sich also vor allem um eine politisch motivierte Organisation. Traditionell sind diese U\&D-Festivals erklärt „unkommerziell“, was genau die Beteiligten darunter verstehen, kann allerdings unterschiedliche sein. Die Finanzierung erfolgt in diesem Fall in erster Linie über den Verkauf von Getränken während der Veranstaltung, da kein Eintritt verlangt wird. Die politische Botschaft und die „roten Linien“ für das Fest werden in der Öffentlichkeit deutlich kommuniziert: Das Festival sei vor allem „unkommerziell, antirassistisch und antisexistisch“ (Homepage und Festivalguide Fall A). Darüber hinaus werden Entscheidungen, die die Organisation betreffen im Konsens auf den wöchentlichen Treffen der ca. 40

5 „Die Idealisten“ und „die Pragmatischen“ als Titel für die beiden Fälle sind keine Typen, sondern lediglich Bezeichnungen, die der plastischeren Darstellung und Kontrastierung der Fälle dienen. 
Hauptverantwortlichen gefällt. Diese Gruppe ist in der Altersstruktur sehr heterogen, die soziale Bindung der Personen an die Organisation wird von den meisten als ausgesprochen eng empfunden. Dies spiegelt sich in den häufig langen Zeiten der Zugehörigkeit oder der Beteiligung über mehrere Generationen hinweg wieder. Neben der eher linkspolitischen Orientierung ist das Festival dadurch geprägt, dass es versucht, sich nicht von der ursprünglichen Ausrichtung und Zielsetzung zu entfernen: So findet eine permanente Diskussion darüber statt „kleiner“ zu werden, wie es zu den Anfängen des Festivals war. Diesem Versuch, sich nicht zu verändern stehen die über die Jahre steigenden Besucherzahlen entgegen. Ökonomischer Erfolg ist für die Organisation nur insofern erstrebenswert, als das er nötig ist, um das Festival im folgenden Jahr wieder stattfinden lassen zu können. Der Erfolg dieses Festivals besteht aus der Sicht der Organisation vor allem darin, den Charakter der Veranstaltung über so viele Jahre beibehalten zu haben. Das Selbstverständnis, als eine Art Sinnbild des Widerstandes gegen Kommerzialisierung und Politikverdrossenheit, lässt eine Beschreibung der Organisation als „die Idealisten“ $\mathrm{zu}$.

\section{Fall B "die Pragmatischen"}

Bis vor elf Jahren war Fall B ebenfalls ein nicht-kommerzielles Festival, das veranstaltet wurde, um Jugendbildungsarbeit zu fördern. Nachdem das Festival jedoch in zwei Jahren zu geringe Einnahmen einbrachte, um die Finanzierung weiter sicher zu stellen, wurde die pragmatische Entscheidung gefällt, den Charakter des Festivals so zu verändern, dass der ökonomische Erfolg stärker in den Vordergrund rückt. Dies hatte einige strategische Veränderung zur Folge, zum Beispiel wurde nun Eintritt verlangt, für viele Aufgaben wurden externe Firmen beauftragt und es wurde ein strategisches Personalmanagement eingeführt. Insgesamt ist das Festival seit diesem Zeitpunkt auf Wachstum ausgelegt, weshalb Ziele wie das Transportieren politischer Botschaften deutlich in den Hintergrund traten. Diese Veränderung hatte den erwünschten Erfolg und das Festival war im Jahr 2011 mit 18.000 verkauften Karten größer und ökonomisch erfolgreicher denn je. Der Geschäftsführer, der sich von Beginn an für das Festival engagierte. teilt mit einigen anderen Beteiligten die Ansicht, dass die Veranstaltung dadurch viel „verloren“ habe. Er sieht jedoch die Entscheidung, die damals gefällt wurde, als alternativlos an. Höchstwahrscheinlich würde es das Festival nach seiner Einschätzung heute ohne diesen Schritt nicht mehr geben. Trotz des aktuellen wirtschaftlichen Erfolgs und dem Bewusstsein für die Notwendigkeiten weiter anpassungsfähig $\mathrm{zu}$ bleiben, ist die Zukunft des Veranstalters allerdings keineswegs gesichert. 


\section{Kompetenzentwicklung}

Informelle Organisationsprinzipien sind in beiden Organisationen vordergründig. Das betrifft vor allem das Feld der Kompetenzentwicklung. Nur in einzelnen Fällen werden Schulungen oder Briefings durchgeführt. Grundsätzlich finden Organisationsprozesse und Lernprozesse in nicht-formalisierten Situationen und vor allem mündlich statt. Der von Dehnbostel (2007) konstatierte hohe Stellenwert von informellem Lernen findet hier seine empirische Entsprechung.

Die Akteure in Fall A bewerten auch die konsensorientierte Organisationsform selbst als lernförderlich:

Konsens ist ganz wichtig. Das ist nicht immer leicht, Das ist manchmal sehr, sehr schwierig. Ist auch etwas, was ich erst ein bisschen lernen musste, was mir auch manchmal schwer fällt, aber was ich sehr, sehr gut finde. Und was ich finde, das kann ich überall mithinnehmen. [...] Und das habe ich gelernt. Das haben die mir beigebracht. Das war vorher für mich nicht nötig, deswegen habe ich das auch nicht anwenden müssen und können und auch das ist sehr wichtig. (Finanzen Fall A)

Partizipative Strukturen (in diesem Fall das Konsensprinzip) können also zur Kompetenzentwicklung der Beteiligten beitragen. Zugleich sind diese in der Lage die erworbenen Kompetenzen in die Organisation (aber auch im Kontext anderer Lebensbereiche) einfließen zu lassen. Dies ist mit der im Fachdiskurs vertretenen Einschätzung vereinbar, dass partizipationsförderliche Strukturen innovationsförderlich sein können (vgl. exempl. Domsch et al. 1995; Keller/Staack 2009).

Nach dem Prinzip „learning by doing“ werden Freiwillige in eine Tätigkeit eingebunden und mit knappen Anweisungen in die Gruppe integriert. Freiwillige Tätigkeiten in den Schlüssel- oder „leitenden“ Positionen werden in beiden Fällen in der Regel nach einigen Jahren übergeben, das heißt dass ein Organisationsmitglied in einer solchen Position einen Nachfolger oder eine Nachfolgerin sucht und beide die Aufgabe für ein oder mehrere Jahre gemeinsam durchführen, um eine Einarbeitung zu gewährleisten. ${ }^{6}$ Vereinzelt gibt es auch Einweisungen (der Freiwilligen) oder Schulungen. So zum Beispiel als im Bundesland von Fall A das Gesetz eingeführt wurde, dass ein gewisser Prozentsatz der Sicherheitskräfte ein Zertifikat für diese Tätigkeit erworben haben müssen. Ein Security-Unternehmen zu beauftragen wäre finanziell und aus politisch-ideologischen Gründen nicht realisierbar gewesen, die externe Schulung ebenso wenig. Daher organisierten ein Jurist und ein Pädagoge selbst einen Lehrgang.

6 Hier zeigen sich „naturwüchsige“ Strukturen, die den pädagogischen Ansätzen der „Rahmung informellen Lernens“ (Molzberger 2007) und somit der pädagogischen Gestaltungsversuchen von informellen Lernprozessen entsprechen. 


\section{Organisationsentwicklung}

Bezeichnend für die Branche und auch für beide Organisationen sind ihre extreme Expansion seit den 1970er Jahren (vgl. Schäfers 2011, S. 39) und der hohe Stellenwert unvorhersehbarer, bzw. unplanbarer Ereignisse (beziehungsweise Kontingenz i.S.v. Ortmann 2009): Bands, die kurzfristig absagen, vereinzelte Akte von Gewalt und Vandalismus auf dem Festivalgelände und als wichtigster Faktor: Das Wetter ${ }^{7}$. Beispielsweise wurde in Fall A 2011 für einige Stunden der Bühnenplatz wegen eines herannahenden Unwetters evakuiert. Dies führte zu erheblichen finanziellen Einbußen.

Prägend für die organisationale Dynamik in Fall A ist der bereits beschriebene Konflikt zwischen sog. „Alt-68ern“, die das Festival so erhalten wollen, wie es zu Beginn war und die der Wahrung der idealistischen Zielsetzung die höchste Priorität beimessen, und Helfern, die das Festival lieber größer werden lassen wollen:

Das eine Lager ist größer, schneller, weiter, höher, so, zu denen zähle ich mich so ein bisschen dazu, und das andere ist halt so ein bisschen: möglichst kleiner, und die zweite Bühne wieder abschaffen und weniger Leute, soll unser Festival sein, und so was. Da gibt es halt mal manchmal Differenzen, so. (Booking, Fall A)

Deshalb muss sich die Organisation permanent über sich selbst und über den Status des „Unkommerziellen“ verständigen. Trotz oder gerade wegen dieses Konflikts, gab es immer wieder organisationale Veränderungen. Auf diese Weise haben sich im Laufe der Jahre Arbeitsbereiche funktional ausdifferenziert, das künstlerische Design wird jedes Jahr verändert und es finden regelmäßige Wochenend-Seminare statt, auf denen Herausforderungen und Lösungsmöglichkeiten für das jeweils nächste Festival diskutiert werden.

In Fall B bestand die größte organisationale Veränderung in der Organisationsgeschichte in dem Schritt zur „Kommerzialisierung“, der mit Outsourcings-, Wachstums- und Vernetzungsprozessen verbunden war:

Und haben dann schon das Ruder rum geworfen und sind einfach stromlinienförmiger und kommerzieller und... Ja, wir haben erst einmal alles raus geworfen, was Geld kostet, aber keinen interessiert auf dem Plakat [...]. Und wir haben das dann so seit 2003 eigentlich hingekriegt, dass wir seit drei Jahren eigentlich ausverkauft sind. Also das heißt, wir sind jetzt ein kommerzielles Festival eigentlich so mehr oder minder. (Geschäftsführer Fall B)

Beide Fälle sind somit Beispiele für Organisationen mit einem hohen Maß an Anpassungs- und (im weiteren Sinne) Innovationsfähigkeit.

7 In Anlehnung an die Akteur-Netzwerk-Theorie (Bellinger/Krieger 2006) wird das Wetter, sowie technische Voraussetzung, das Gelände etc. als „Akteur“ begriffen, also als etwas, was sozial relevant wird. Beispiele von tragischen Unglücken bei Festivals belegen den Stellenwert solcher Rahmenbedingungen für die Organisation. 


\section{Fazit und Ausblick: Kompetenzentwicklung und Organisationsentwicklung}

Die Berufs- und Wirtschaftspädagogik hat in Bezug auf Organisationen einen Gestaltungsanspruch, der allerdings eine Kenntnis der jeweils vorhandenen Strukturen, Ressourcen und Herausforderungen voraussetzt. Die hier vorgestellte Studie ist eine Exploration eines potentiellen pädagogischen Handlungsfeldes. Sie vergleicht mit ethnographischen Methoden zwei Veranstalter von Musikfestivals im Hinblick auf kommunikative Strukturen, die Kompetenz- an Organisationsentwicklung anschließen und umgekehrt. In beiden Organisationen werden Erfahrungen und Kompetenzen, die bei diesem Festival erworben wurden, unabhängig vom sozialen Status als natürliche Autorität wirksam.

Auch wird nachvollziehbar, welche Voraussetzungen dazu führen, dass innovative „kleine und große Ideen“ (Tarde 2003, S. 26), die auf Grundlage von individuellen Kompetenzen entstehen, organisational wirksam werden. Allein eine Idee zu haben, reicht allerdings nicht aus, damit diese letzten Endes zu Innovation führen kann. Sie muss dafür auch kommunikative Anschlussmöglichkeiten (i.S.v. Luhmann 1994) erzeugen. Ob das passiert, hängt zum einen von den Fähigkeiten und dem Willen der Person ab, eine Idee zu äußern, und zum anderen von den organisationalen Strukturen, die Partizipation entweder fördern oder hemmen. Der Rahmen für (informelle) Kompetenzentwicklung und die Möglichkeit von Partizipation bilden also Voraussetzungen für die erfolgreiche Verknüpfung von individuellen und organisationalen Veränderungsprozessen, die wiederum die Voraussetzung für die Innovationsfähigkeit einer Organisation darstellt.

\section{Literatur}

Anand, N.; Gardner, H. K.; Morris, T. (2007): Knowledge-based Innovation: Emergence and embedding of new practice areas in management consulting firms. In: Academy of Management Journal, Jg. 50, H. 2, S. 406-428.

Arnold, R. (1996): Systemlernen und Berufsbildung. In H. Geißler (Hrsg.), Arbeit, Lernen und Organisation. Ein Handbuch. Weinheim, S. 371-383.

Arnold, Rolf; Lermen, Markus (2005): Lernen, Bildung und Kompetenzentwicklung. Neuere Entwicklungen in Erwachsenenbildung und Weiterbildung. Weinheim und München.

Bartels, R. (2001): Kompetenzentwicklung mit bestimmten Besonderheiten der betrieblichen Mitgestaltung von Weiterbildung im Zusammenhang mit neuer Arbeitsorganisation für Betriebs- und Personalräte im Dienstleistungssektor. Dissertation vorgelegt an der TU Darmstadt. 
Bellinger, Andréa; Krieger, David J. (Hg.) (2006): ANThology. Ein einführendes Handbuch zur Akteur-Netzwerk-Theorie. Bielefeld.

Bethscheider, M. et al (2007): Berufliche Kompetenzentwicklung - Ausgewählte Arbeiten des BIBB. In: Berufsbildung in Wissenschaft und Praxis, Jg. 36, H. 6, S. $15-19$.

Böhle, F.; Bolte, A. (2002): Die Entdeckung des Informellen. Der schwierige Umgang mit Kooperation im Arbeitsalltag. München.

Bohlinger, S. \&. Münk D. (2008): Verschiedene Blickwinkel: Kompetenz, competence, compétence -. Ein Begriff, drei Auffassungen. Weiterbildung. In: Weiterbildung, Jg. 19, H. 1, S. 36-39.

Bude, H. (2008): Die Kunst der Interpretation. In: Flick, U.; von Kardorff, E.; Steinke, I. (Hrsg.): Qualitative Forschung 6. Hamburg, S. 569-578.

Dehnbostel, Peter (2007): Lernen im Prozess der Arbeit. Münster.

Dietzen, A. (2004): Beraterwissen und Qualifikationsentwicklung. Bielefeld.

Domsch, Michael E.; Ladwig, Désirée H.; Siemers, Sven H. A. (1995): Innovation durch Partizipation. Eine erfolgversprechende Strategie für den Mittelstand. Stuttgart: Schäffer-Poeschel (Management von Forschung, Entwicklung und Innovation, 14).

Geertz, C. (1983): Dichte Beschreibung. Frankfurt a. M..

Geißler, Harald (Hg.) (1996): Arbeit, Lernen und Organisation. Ein Handbuch. Weinheim und München.

Gnahs, Dieter (2007): Kompetenzen - Erwerb, Erfassung, Instrumente. Bielefeld: Bertelsmann (Studientexte für Erwachsenenbildung).

Hartz, Stefanie (2004): Biografizität und Professionalität. Eine Fallstudie zur Bedeutung von Aneignungsprozessen in organisatorischen Modernisierungsstrategien. Wiesbaden.

Horsch, Jürgen (2003): Innovations- und Projektmanagement. Von der strategischen Konzeption bis zur operativen Umsetzung. 1. Aufl. Wiesbaden.

Keller, Andreas; Staack, Sonja (2009): Innovation durch Partizipation. Steuerung von Hochschulen und Forschungseinrichtungen im 21. Jahrhundert. Bielefeld.

Latour, B. (2007): Eine neue Soziologie für eine neue Gesellschaft. Frankfurt a. M..

Luhmann, N. (1987): Soziale Systeme. Grundriß einer allgemeinen Theorie. 4. Aufl.

Luhmann, N.; Baecker, D. (2009): Einführung in die Systemtheorie. 5. Aufl.

Meyer, R., Elsholz, U. (2009): Berufliche und betriebliche Weiterbildung als Gegenstand der Berufs- und Wirtschaftspädagogik. Desiderata und neue Perspektiven für Theorie und Forschung. In: bwpat, H. 16.

Molzberger, G. (2007): Rahmungen informellen Lernens. Zur Erschließung neuer Lern- und Weiterbildungsperspektiven. Wiesbaden.

Molzberger, Gabriele (2007): Rahmungen informellen Lernens. Zur Erschließung neuer Lern- und Weiterbildungsperspektiven. Hamburg.

Ortmann, G. (2009): Management in der Hypermoderne. Kontingenz und Entscheidung. Wiesbaden.

Reichertz, J. (2000): Abduktion, Deduktion und Induktion in der qualitativen Forschung. In: Flick, U.; Kartoff, E. von; Steinke, I. (Hrsg.): Qualitative Forschung. Ein Handbuch, Hamburg, S. 276-286.

Schäfers, Bernhard (2011): Architekturen für die Stadt als Ort der Feste, Spiele und Events. In: Betz, Gregor; Hitzler, Ronald; Pfadenhauer, Michaela (Hg.): Urbane Events. Wiesbaden, S. 27-42. 
Staudt, E. (2002): Kompetenzentwicklung und Innovation. Die Rolle der Kompetenz bei Organisations-, Unternehmens- und Regionalentwicklung. (Edition QUEM, 14).

Staudt, Erich; Kailer, Norbert; Kottmann, Marcus, et al. (Hg.) (2002): Kompetenzentwicklung und Innovation. Die Rolle der Kompetenz bei Organisations-, Unternehmens- und Regionalentwicklung.

Tarde, G. (2003): Die Gesetze der Nachahmung. Frankfurt a. M..

Vogd, W. (2009): Rekonstruktive Organisationsforschung. Qualitative Methodologie und theoretische Integration - eine Einführung. Opladen.

Wilkens, U. (2004): Management von Arbeitskraftunternehmern. Psychologische Vertragsbeziehungen und Perspektiven für die Arbeitskräftepolitik in wissensintensiven Organisationen. Wiesbaden.

Wolcott, H. F (2008): Ethnography. A way of seeing. 2.Aufl. Lanham. 


\title{
Karrieren und Weiterbildung von Ingenieuren
}

\author{
Martin Kröll
}

\section{Ausgangspunkt und Forschungsfrage}

Vor dem Hintergrund der zunehmenden Globalisierung, des demographischen Wandels und der verschärften Wettbewerbssituation werden Faktoren, die den beruflichen Lebenslauf insbesondere von Ingenieuren beeinflussen, immer komplexer, vielfältiger und undurchsichtiger. Das traditionelle Karriereverständnis, welches den hierarchischen Aufstieg in einer Unternehmung über verschiedene Führungspositionen umfasst (sog. Führungskarriere), löst sich zunehmend auf oder wird durch neue Karrierewege ergänzt. Im Berufsfeld der Ingenieure wird neben der klassischen Führungslaufbahn vor allem die gleichwertige Etablierung von Fach- und Projektlaufbahnen diskutiert. Die vorliegende Studie analysiert Karriereplanungen und Weiterbildungsaktivitäten von 544 Ingenieuren und erörtert, inwieweit Erwerbsbiographien gerade für die Berufsgruppe der Ingenieure überhaupt noch vorausgeplant werden können. Außerdem wird untersucht, ob Ingenieure sich bei ihren Kompetenzentwicklungsaktivitäten von bestimmten Karrierewegen und -vorstellungen leiten lassen. Die forschungsleitende Fragestellung kann wie folgt zusammengefasst werden: Woran orientieren sich die Ingenieure bei der Gestaltung ihrer Erwerbsbiographie und welche Auswirkungen hat dies auf das Weiterbildungsverhalten?

\section{Theoretischer Bezugsrahmen, Begründung der Hypothesen}

Lange Zeit galt die Führungskarriere (FÜ-Karriere) als die Leitkarriere für Ingenieure, an der sich ihre Laufbahnplanung und ihre Kompetenzentwicklungsaktivitäten ausrichten sollten. Darunter wird eine ,traditionelle Karriere in Form eines kontinuierlichen ranghierarchischen Aufstiegs bei entsprechenden Qualifikationen“"verstanden (Thom, 2008, S. 11). Die Erkenntnis, dass nicht jeder Ingenieur zur Übernahme von Führungsverantwortung geeignet ist, überrascht nicht. Ausgehend von dem in jüngster Zeit in den Unternehmen favorisierten Konzept der flachen Hierarchien ist es darüber hinaus unrealistisch, dass ein großer Teil der Ingenieure in den Unternehmen 
die Position einer Führungskraft übernehmen kann. Diese Umstände bewirken, dass die Nachfrage nach FÜ-Karrieren größer ist als das Angebot entsprechender Positionen in Unternehmen.

Während lange Zeit in der Karriereforschung vor allem duale Karrieresysteme (= Führungs- und Fachlaufbahnen, Kunz, 2005) diskutiert wurden, weisen u.a. Majer und Mayrhofer (2007) auf sogenannte triale Karrieresysteme hin. Diese bestehen aus den drei Karrierefeldern der Führungskarriere, Fachkarriere (FA-Karriere) sowie Projektkarriere (PROJ-Karriere). FA-Karrieren werden in Anlehnung an Thom (2008, S. 252) als Karrierewege definiert, die einen Aufstieg über verschiedene an Fachwissen geknüpfte Positionen umfassen, wobei spezifisches Wissen im Gegensatz zur FÜund PROJ-Karriere im Vordergrund steht und Personalverantwortung nicht oder nur in geringem Maße übernommen wird. Zudem spielen Steuerungsaufgaben in dieser Laufbahn eine geringe Rolle. Die PROJ-Karriere dagegen umfasst projektbezogene Führungs- und Planungsaufgaben und kann somit als Mischung aus klassischer FÜ- und spezifischer FA-Karriere definiert werden. Die Positionen im Rahmen einer PROJ-Karriere sind zumeist zeitlich determiniert, was eine höhere Flexibilität der Laufbahnentwicklung impliziert. In den letzten Jahren gewann dieser Weg an Beliebtheit und wurde von einigen Forschern sogar als eigener Berufsstand bezeichnet. Der momentane Stand der Forschung weist allerdings darauf hin, dass eine Gleichwertigkeit von PROJ- und FÜ-Karriere nicht gegeben ist. CorriaSimpson et al. (2008) nennen insbesondere das Fehlen eines definierten Karrierepfads und formaler Betreuungsprogramme als Nachteile einer PROJKarriere. Auch Status und Einflussmöglichkeiten scheinen in der PROJKarriere noch nicht in dem von Ingenieuren erwünschten Maße erreichbar zu sein (Hodgson, Paton \& Cicmil, 2011). Ähnliche Nachteile sind auch für die FA-Karriere im Vergleich zur FÜ-Karriere zu beobachten. Dieses Ungleichgewicht birgt die Gefahr, dass Organisationsmitglieder sich für eine FÜKarriere entscheiden, obwohl diese nicht ihren Kompetenzen und primären Interessen entspricht. Es ist anzunehmen, dass die mangelnde Unterstützung durch Unternehmen zu einem höheren Ausmaß an Unzufriedenheit bei den Ingenieuren führt, die eine FA- oder PROJ-Karriere eingeschlagen haben. Basierend darauf wird folgende Hypothese $(\mathrm{H})$ aufgestellt:

H1: Ingenieure, die eine FÜ-Karriere eingeschlagen haben, sind im Vergleich zu Ingenieuren mit FA- oder PROJ-Karriere (1) mit ihrer beruflichen Situation zufriedener und (2) beurteilen ihre beruflichen Perspektiven positiver.

In Zeiten eines steigenden Fachkräftemangels in technischen Berufen ist es von zentraler Bedeutung für Unternehmen, Ingenieure langfristig zu binden. Aus diesem Grund ist es von Interesse zu analysieren, aus welchen Gründen Ingenieure ihren Karriereweg wechseln bzw. das Unternehmen verlassen. Auf der Basis der Equity-Theorie (Adams, 1965; Skiba \& Rosenberg,. 2011), 
die davon ausgeht, dass Individuen gerechte Gegenleistungen für ihren Arbeitseinsatz erwarten, kann angenommen werden, dass unterforderte Ingenieure sich nicht ihrem Potenzial entsprechend gefördert fühlen und somit ein Ungerechtigkeitsempfinden bei ihnen entsteht. Es ist vorstellbar, dass sie sich überqualifiziert bzw. unterbezahlt fühlen und eine Position mit mehr Anforderungen (und dementsprechend auch höherem Gehalt) wünschen.

Ergänzend kann die Argumentation durch die Anreiz-Beitrags-Theorie (March \& Simon, 1958) gestützt werden. Demnach erhalten Mitarbeiter von der Organisation Anreize und leisten im Gegenzug dafür Beiträge. Unzufriedenheit und letztendlich eine Beendigung der Arbeitsbeziehung können dann eintreten, wenn die gewährten Anreize die geleisteten Beiträge unterschreiten. Im Falle hoch qualifizierter, unterforderter Arbeitnehmer ist dies naheliegend: Sie können leicht das Empfinden haben, dass die eingebrachten Beiträge (in Form von Kompetenzen und Qualifikationen) die erhaltenen Anreize (in Form einer interessanten, fordernden Tätigkeit oder potenzialadäquaten Bezahlung) übersteigen. Einhergehend mit diesen Überlegungen konnten Gerstenfeld und Rosica (1970) zeigen, dass die Hauptgründe für einen Jobwechsel bei Ingenieuren u.a. in interessanteren Tätigkeiten sowie der Möglichkeit zu einem persönlichen Vorwärtskommen begründet sind.

H2: Unterforderte Ingenieure sind zu einem Wechsel im Rahmen ihrer Erwerbsbiographie eher bereit als überforderte Ingenieure

Gerade für Ingenieure wird die Bedeutung lebenslangen Lernens immer wieder hervorgehoben, da sie in besonderer Weise am technischen Fortschritt mitwirken und davon betroffen sind sowie in größerem Maße Gefahr laufen, dass ihr Wissen veraltet. Dabei stellt sich jedoch die Frage, auf welche Weise Lernen geschehen soll und welche Art von Wissen dabei erworben werden soll. In der wissenschaftlichen Auseinandersetzung wird die Differenzierung zwischen explizitem und implizitem Wissen als grundlegend angesehen ( $\mathrm{z}$. B. Nonaka \& Takeuchi, 1997; Lam, 2000). Explizites Wissen ist demnach formal artikulierbares Wissen, das beispielsweise auf Theorien basiert und auch als „Verstandeswissen“ bezeichnet werden kann (Nonaka \& Takeuchi 1997). Implizites Wissen dagegen ist nicht ohne weiteres sprachlich artikulierbar, kontextspezifisch und oftmals seinem Inhaber nicht bewusst. Es kann z.B. „Erfahrungswissen“ sein oder gelernte Routinen und Verhaltensweisen umfassen. Wenn es um die Wettbewerbsfähigkeit von Organisationen sowie um die Gewährleistung der Qualität von Produkten oder Dienstleistungen geht, dann wird dem implizitem Wissen, das z.B. in Fertigkeiten, Techniken, Know-How und Routinen einfließt, eine zentrale Rolle zugewiesen. Lam (2000) argumentiert, dass Innovationsfähigkeit und Lernen in einer Organisation maßgeblich von der Generierung und Weitergabe impliziten Wissens abhängten. Der Erwerb impliziten Wissens könne jedoch nur durch praktische Erfahrung in dem jeweilig relevanten Arbeitskontext erfolgen (,Learning by Doing“; Nonaka \& Takeuchi, S. 82). Die Weitergabe dieser Art von 
Wissen erfolgt oftmals erfahrungs- und handlungsorientiert sowie abhängig von sozialer Interaktion. Es ist anzunehmen, dass Ingenieure die Bedeutung impliziten Wissens für die Generierung von Wettbewerbsvorteilen für die Unternehmung und somit auch für die eigene Karriere einschätzen können und „erfahren“ haben. Basierend auf dieser Annahme werden Ingenieure diejenigen Weiterbildungsformen bevorzugen, die den Erwerb neuen impliziten Wissens möglich machen.

H3: Ingenieure weisen, unabhängig von dem gewählten Karriereweg, arbeitsplatznahen WB-Formen eine größere Rolle zu als externen WBFormen.

Karrieren werden immer komplexer - so Strunk (2009) vor dem Hintergrund der Theorien nichtlinearer dynamischer Systeme, die davon ausgeht, dass Systemverhalten über längere Zeit von nicht vorhersagbaren Wechselwirkungen der Elemente beeinflusst wird. Von dieser Entwicklung sind eher jüngere als ältere Ingenieure betroffen, die ihre Karriereplanung erst noch vor sich haben und damit in besonderer Weise herausgefordert sind gegebenenfalls alternative Karrierewege einzuschlagen. Die PROJ-Karriere kann dabei als alternativer Career Pathway im Sinne von Strunk interpretiert werden, da sie eine erhöhte Flexibilität aufweist und im späteren Erwerbsleben die Möglichkeit bietet, eine FÜ- oder eine FA-Karriere einzuschlagen oder die PROJ-Karriere weiter zu verfolgen. Für jüngere Ingenieure erscheint es vor dem Hintergrund der Erkenntnis der zunehmenden Komplexität der Karriereentwicklung sinnvoll, sich den Herausforderungen einer ständig veränderten Umwelt zu stellen und alternative Karrierewege anzustreben:

H4: Jüngere Ingenieure orientieren sich neben den alten Karrierewegen (FAund FÜ-Karriere) in Bezug auf ihre zukünftige Karriereplanung vermehrt an einer alternativen Karrieregestaltung (hier operationalisiert durch PROJ-Karriere).

Frauen sind im Ingenieurberuf deutlich unterrepräsentiert und haben häufig mit Vorurteilen zu kämpfen. Nach Herrmann (2004, S. 262) werden Ingenieurinnen von Unternehmen häufiger für Projekttätigkeiten eingesetzt. In einer Befragung von 151 Männern und 130 Frauen aus dem mittleren Management und dem Personalbereich konnte Funken (nach Nitsche, 2011) zudem zeigen, dass Managerinnen sich häufiger in einer PROJ-Karriere und seltener in FÜ-Laufbahnen engagieren. Deswegen wird angenommen:

H5: Ingenieurinnen wählen häufiger als Ingenieure eine Projektkarriere und streben diese auch häufiger als zukünftigen Karriereweg an. 


\section{Methode}

Der vorliegende Beitrag stützt sich auf eine Online-Befragung (42 Fragen) von Ingenieuren/-innen (88\% Männer) aus dem Jahre 2008, die vom VDI koordiniert wurde (Lienert 2010). Die Datenbasis bildeten 544 ausgefüllte Fragebögen (Rücklaufquote 17\%). Für die Bestimmung von Variablenausprägungen bei den verschiedenen Fragen gilt generell, dass sämtliche Variablen auf Likert-Skalen mit sechs abgestuften Antwortvorgaben, die von „stimme stark zu“

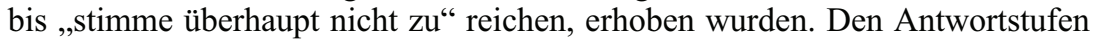
wurden Zahlen von 1 bis 6 zugeordnet, wobei niedrige Werte eine höhere $\mathrm{Zu}$ stimmung der jeweiligen Variablen bedeuten. Die Auswertung der Datensätze wurde mit dem Statistikprogramm SPSS vorgenommen (T-Tests, ANOVA, Chi-Quadrat-Tests, Faktorenanalysen (Hauptkomponentenanalyse, VarimaxRotation), Kreuztabellen, Korrelationskoeffizienten nach Pearson). Tab. 1 gibt einen Überblick über demographische Daten der Befragten und wird durch Tab. 2 ergänzt, die sich auf Karrierewege bezieht.

Tabelle 1: Deskriptive Beschreibung der Stichprobe

\begin{tabular}{|c|c|c|c|}
\hline \multicolumn{2}{|l|}{ Variable } & \multirow{2}{*}{$\frac{\mathbf{N}}{145}$} & \multirow{2}{*}{$\begin{array}{c}\text { Prozentualer Anteil } \\
27 \%\end{array}$} \\
\hline & $21-30$ & & \\
\hline Alter & $31-40$ & 186 & $35 \%$ \\
\hline & $41-50$ & 130 & $24 \%$ \\
\hline & $51-60$ & 55 & $10 \%$ \\
\hline & über 60 & 18 & $3 \%$ \\
\hline \multirow{4}{*}{ Höchster Bildungsabschluss } & Diplom Fachhoschschule & 239 & $45 \%$ \\
\hline & Diplom Universität & 190 & $36 \%$ \\
\hline & Promotion & 61 & $11 \%$ \\
\hline & Sonstige (Bachelor, Master usw.) & 54 & $8 \%$ \\
\hline \multirow{6}{*}{ Tätigkeitsfeld } & Entwicklung & 126 & $24 \%$ \\
\hline & Vertrieb und Marketing & 61 & $12 \%$ \\
\hline & Fertigung und Produktion & 53 & $10 \%$ \\
\hline & Konstruktion & 45 & $9 \%$ \\
\hline & Forschung & 43 & $8 \%$ \\
\hline & $\begin{array}{l}\text { Anderer Bereich (z.B. Verwaltung, } \\
\text { Beratung, Logistik, Geschäftsfüh- } \\
\text { rung) }\end{array}$ & 216 & $37 \%$ \\
\hline
\end{tabular}


Tabelle 2: Eingeschlagener / für die Zukunft gewünschter / von Unternehmen gewünschter Karriereweg der befragten Ingenieure

( $N=534$, 10 fehlende Angaben)

\begin{tabular}{cccc}
\hline Karriereweg & Eingeschlagen & $\begin{array}{c}\text { Für die Zukunft } \\
\text { angestrebt }\end{array}$ & $\begin{array}{c}\text { Von Unternehmen } \\
\text { gewünscht }\end{array}$ \\
\hline Fachkarriere & $36,7 \%$ & $21,8 \%$ & $38,4 \%$ \\
Führungkarriere & $33,0 \%$ & $56,6 \%$ & $23,3 \%$ \\
Projektkarriere & $27,5 \%$ & $19,5 \%$ & $36,3 \%$
\end{tabular}

\section{Ergebnisse der Studie}

Zwar gibt es Kriterien, um einer Erwerbsbiographie einen der drei Karrierewege „FÜ-, FA- bzw. PROJ-Karriere“ zuzuordnen, in der vorliegenden Befragung wurden die Ingenieure aber um eine Selbsteinschätzung ihres jeweiligen Karrieretyps zum heutigen Zeitpunkt sowie um eine Darlegung ihrer zukünftigen Planung gebeten. Diese Vorgehensweise erweist sich als vorteilhaft, da so die Selbstkategorisierung der betroffenen Akteure bzw. Akteursgruppen, die ihr Handeln entscheidend beeinflussen, als Ausgangspunkt für die weiteren Überlegungen herangezogen werden. Es konnte festgestellt werden, dass die drei Karrieretypen in Bezug auf die Bedeutung verschiedener Karriereindikatoren sowie präferierter WB-Themen voneinander differieren. FÜ-Karriere-Ingenieure messen bspw. Personalverantwortung als Indikator einer erfolgreichen Karriere eine höhere Bedeutung zu $(F(2,507)$ $=13,58, \mathrm{p}<.001$, Post-hoc Bonferroni Tests: $\mathrm{p}<.01$ für beide Gruppen). Ingenieure mit FÜ-Karriere unterscheiden sich zudem signifikant von FAKarriereingenieuren in einer höheren Bedeutungseinschätzung des Aufstiegs in der Unternehmensstruktur $(\mathrm{F}(2,505)=4,67, \mathrm{p}<.01$, Post-hoc Bonferroni Test $\mathrm{p}<.01$ für beide Gruppen). Dagegen ist es für FA-Karriereingenieure gegenüber den anderen Ingenieursgruppen signifikant wichtiger, anerkannter Experte im eigenen Feld zu sein $(\mathrm{F}(2,509)=6,93, \mathrm{p}<.001)$. FÜ-KarriereIngenieure halten das Kommunikationsverhalten $(\mathrm{M}=1,43, \mathrm{SD}=0,58)$, gefolgt von Führungskompetenzen $(\mathrm{M}=1,44, \mathrm{SD}=0,64)$ und Teamentwicklung $(\mathrm{M}=1,47, \mathrm{SD}=0,65)$ als WB-Thema besonders relevant für ihre Karriere. Demgegenüber stehen für die, die eine FA-Karriere verfolgen, technische Fachkenntnisse an erster Stelle $(\mathrm{M}=1,41 ; \mathrm{SD}=0,66)$. Ingenieure, die sich für eine PROJ-Karriere entschieden haben, stufen das WB-Thema Projektmanagement als zentral ein $(\mathrm{M}=1,41, \mathrm{SD}=0,66)$. Post-hoc Scheffé Tests ergeben, dass sich alle drei Gruppen in Bezug auf Fach- und Wirtschaftskenntnisse, Teamentwicklung und Führungskenntnisse signifikant unterscheiden $(\mathrm{p}<.05)$. Die vorausgegangen Erkenntnisse legen nahe, dass die Differenzierung zwischen FÜ-, FA- und PROJ-Karriere als ausreichend be- 
gründet angesehen werden kann und sich diese für die weitere Auseinandersetzung mit der angesprochenen Problematik als tragfähig erweist.

In Bezug auf Hypothese 1 zeigt sich, dass Ingenieure, die sich für eine FÜ-Karriere entschieden haben, mit ihrer beruflichen Situation deutlich zufriedener sind $(\mathrm{N}=172, \mathrm{M}=2,76 ; \mathrm{SD}=1,00)$ als diejenigen, die eine FAKarriere $(\mathrm{N}=186, \mathrm{M}=3,15, \mathrm{SD}=1,01)$ oder PROJ-Karriere $(\mathrm{N}=137, \mathrm{M}=3,13$, $\mathrm{SD}=1,00)$ gewählt haben. Ein univariater Mittelwertvergleich weist nach, dass die Gruppenunterschiede signifikant sind $(\mathrm{F}(3,505)=5,31 ; \mathrm{p}<0,01)$. Die Durchführung von Bonferroni Post-hoc Tests bestätigt, dass Ingenieure mit FÜ-Karriere signifikant zufriedener als Fach- und Projektlaufbahningenieure sind $(\mathrm{p}<0,01)$. Weiterhin wurde die Frage ,Wie beurteilen Sie ihre beruflichen Perspektiven?" ausgewertet ( $1=$ sehr gut, $6=$ ungenügend). Ingenieure, die eine FÜ-Karriere verfolgen, stufen ihre beruflichen Perspektiven als gut ein $(M=2,06, S D=0,95)$. Diejenigen, die FA- und PROJKarrieren verfolgen, stufen ihre beruflichen Perspektiven zwischen ,gut“ und „befriedigend" ein (FA-Karriere: $\mathrm{M}=2,56, \quad \mathrm{SD}=1,09$; PROJ-Karriere: $\mathrm{M}=2,37, \mathrm{SD}=0,95)$. Die Unterschiede zwischen FÜ-Karriere und FAKarriere $(\mathrm{U}=12375, \mathrm{r}=-.25)$ sowie PROJ-Karriere $(\mathrm{U}=9872, \mathrm{r}=-.19)$ sind signifikant.

Zur Überprüfung von $\mathrm{H} 2$ wurden die Antworten auf die Frage „Insgesamt gesehen, wie fühlen Sie sich in Ihrer Arbeit?" analysiert (sechsstufige Likert-Skala, $1=$ sehr deutlich überfordert; $6=$ sehr deutlich unterfordert). Die Ingenieure wurden in zwei Gruppen aufgeteilt: die tendenziell Überforderten (Antwortmöglichkeit 1-3) und die eher Unterforderten (Antwortmöglichkeit 4-6). Diese Gruppen wurden in Bezug gesetzt zur Planung eines Wechsels (zwei Antwortmöglichkeiten: Wechsel geplant vs. Wechsel nicht geplant). Zunächst wurde sichergestellt, dass sich Ingenieure je nach Laufbahn (FÜ-, FA- oder PROJ-Karriere) nicht in ihrer Wechselbereitschaft $(\chi 2=5,29$, $\mathrm{p}>0,05)$ oder in der Zugehörigkeit zur Unter- bzw. Überforderungsgruppe $(\chi 2=$ $3,43, p>0,05)$ unterscheiden. Dann wurde mittels Kreuztabellen die unterschiedliche Ausprägung der Wechselbereitschaft und der Über- bzw. Unterforderung überprüft (s. Tab. 3). Es zeigt sich, dass mehr unterforderte Ingenieure einen Wechsel planen als überforderte. Besonders ausgeprägt ist dieser Unterschied in der Gruppe der Führungskräfte: Hier sind $69 \%$ der Wechselwilligen unterfordert. Bei den Ingenieuren, die sich für eine FA- oder PROJ-Karriere entschieden haben, sind jeweils $62 \%$ der Wechselwilligen unterfordert.

Die Frage „Welche Formen der WB nutzen Sie für Ihre Karriere?“ wurde zur Überprüfung der H3 bewertet (Likert-Skala, $1=$ sehr relevant $-6=$ stark irrelevant). Tab. 4 zeigt die Mittelwerte und Standardabweichungen sowohl für die Gesamtstichprobe als auch aufgegliedert nach den drei unterschiedl. Karrierewegen sowie signifikante Gruppenunterschiede (ANOVA). 
Tabelle 3: Über-und Unterforderung und Bereitschaft zum Wechsel in Abhängigkeit vom Karriereweg (\%-Angaben bezogen auf Wechselplanung)

\begin{tabular}{|c|c|c|c|c|c|c|c|c|}
\hline & \multicolumn{2}{|c|}{ Alle Karrierewege } & \multicolumn{2}{c|}{ FÜ-Karriere } & \multicolumn{2}{c|}{ FA-Karriere } & \multicolumn{2}{c|}{ PROJ-Karriere } \\
\hline & Überfordert & Unterfordert & Überfordert & Unterfordert & Überfordert & Unterfordert & Überfordert & Unterfordert \\
\hline $\begin{array}{c}\text { Wechsel } \\
\text { geplant }\end{array}$ & $98(36 \%)$ & $177(64 \%)$ & $27(31 \%)$ & $60(69 \%)$ & $37(38 \%)$ & $60(62 \%)$ & $33(38 \%)$ & $53(62 \%)$ \\
\hline $\begin{array}{c}\text { Kein } \\
\text { Wechsel }\end{array}$ & $71(55 \%)$ & $87(45 \%)$ & $26(49 \%)$ & $27(51 \%)$ & $26(38 \%)$ & $43(62 \%)$ & $19(56 \%)$ & $15(44 \%)$ \\
\hline $\begin{array}{c}\text { Chi- } \\
\text { Quadrat }\end{array}$ & \multicolumn{2}{|c|}{$\chi^{2}=3,65, \mathrm{p}=.06$} & \multicolumn{2}{|c|}{$\chi^{2}=4,55, \mathrm{p}<.05 *$} & $\chi^{2}=0,00, \mathrm{p}=.95$ & $\chi^{2}=3,04, \mathrm{p}=.08$ \\
\hline
\end{tabular}

Für die künftige Karriereentwicklung wurde „Learning by Doing“ mit Abstand am höchsten eingeschätzt $(\mathrm{M}=1,65 ; \mathrm{SD}=0,81)$. Einem MBA, MasterStudium oder einer Promotion werden eher geringe WB-Relevanzen zugemessen. Signifikante Gruppenunterschiede ergaben sich im Hinblick auf „Kurse/Seminare bei externen Bildungseinrichtungen“ $(\mathrm{F}(2,507)=3,67$, $\mathrm{p}<.05$ ) und „Qualitätszirkel, Workshops, Beteiligungs- und Arbeitsgruppen“" $(\mathrm{F}(2,487)=3,20, \mathrm{p}<.05)$.

Tabelle 4: Mittelwerte und Standardabweichungen der neun zu bewertenden WBFormen sowie Gruppenunterschiede zwischen FÜ-, FA- und PROJKarriere

\begin{tabular}{|c|c|c|c|c|c|c|c|c|c|c|}
\hline \multirow[b]{2}{*}{ Item } & \multicolumn{4}{|c|}{ Mittelwert } & \multicolumn{4}{|c|}{ Standardabweichung } & \multicolumn{2}{|c|}{$\begin{array}{c}\text { Gruppen- } \\
\text { unterschiede }\end{array}$} \\
\hline & Gesamt & Fach & Führ. & Projek & Gesamt & Fach & Führ & Projekt & $\mathrm{F}$ & Signifikanz \\
\hline Arbeitsplatznahe Formen & & & & & & & & & & \\
\hline Learning by Doing & 1,65 & 1,68 & 1,66 & 1,58 & 0,81 & 0,88 & 0,71 & 0,81 & 0,74 & ,480 \\
\hline Unterweisung am Arbeitsplatz & 3,41 & 3,26 & 3,51 & 3,47 & 1,44 & 1,42 & 1,41 & 1,49 & 1,54 & ,216 \\
\hline $\begin{array}{l}\text { Qualitätszirkel, Workshops, } \\
\text { Beteiligungs-, Arbeitsgruppen }\end{array}$ & 3,47 & 3,60 & 3,20 & 3,50 & 1,54 & 1,49 & 1,51 & 1,57 & 3,20 &, $042 *$ \\
\hline Arbeitsplatzferne Formen & & & & & & & & & & \\
\hline $\begin{array}{l}\text { Kurse/Seminare bei externen } \\
\text { Bildungseinrichtungen }\end{array}$ & 2,51 & 2,52 & 2,32 & 2,67 & 1,17 & 1,18 & 1,05 & 1,27 & 3,67 &, $026^{*}$ \\
\hline $\begin{array}{l}\text { Besuch von Fachmessen / } \\
\text { Fachkongressen }\end{array}$ & 2,81 & 2,88 & 2,72 & 2,86 & 1,26 & 1,24 & 1,21 & 1,34 & 0,80 &, 451 \\
\hline E-Learning & 3,81 & 3,73 & 3,91 & 3,79 & 1,55 & 1,62 & 1,52 & 1,49 & 0,62 &, 537 \\
\hline $\begin{array}{l}\text { Master of } \\
\text { Science/Engineering }\end{array}$ & 5,16 & 5,10 & 5,09 & 5,27 & 1,61 & 1,62 & 1,67 & 1,55 & 0,49 & ,614 \\
\hline MBA & 5,38 & 5,44 & 5,36 & 5,27 & 1,34 & 1,16 & 1,45 & 1,47 & 0,57 &, 565 \\
\hline Promotion & 4,90 & 4,90 & 4,87 & 4,97 & 1,83 & 1,80 & 1,86 & 1,85 & 0,09 & ,916 \\
\hline
\end{tabular}


Die Durchführung von Post-hoc Scheffé-Tests zeigt, dass „Kursen/ Seminaren bei externen Bildungseinrichtungen“"von Führungskräften eine signifikant relevantere Rolle als von Ingenieuren mit PROJ-Karrieren zugemessen wird. Qualitätszirkel usw. werden ebenfalls von Führungskräften als wichtig eingeschätzt.

Zur Untersuchung von H4 wurde die Frage nach dem zukünftig geplanten Karriereweg ausgewertet (s. Tab. 5). Es ist ersichtlich, dass der Wunsch einer FÜ-Karriere mit zunehmendem Alter abnimmt und mit $65 \%$ am deutlichsten in der Gruppe der jungen Ingenieure (21-30 Jahre) vorhanden ist. Während bei den PROJ-Karrieren eine ähnliche Entwicklung deutlich wird, lässt sich eine signifikant gegenläufige Tendenz bei dem Wunsch nach FAKarrieren beobachten: Je älter die Ingenieure, desto häufiger wollen sie eine FA-Karriere einschlagen (21-30-jährige: 11\%; 51- über 60-jährige: 33\%).

Tabelle 5: Geplanter Karriereweg der Ingenieure, aufgeteilt nach Altersklassen

\begin{tabular}{|c|c|c|c|c|}
\hline Altersklasse & FA-Karriere & FÜ-Karriere & PROJ-Karriere & Gesamt \\
\hline $21-30$ & $16(11 \%)$ & $92(65 \%)$ & $33(24 \%)$ & 141 \\
\hline $31-40$ & $43(24 \%)$ & $108(59 \%)$ & $31(17 \%)$ & 182 \\
\hline $41-50$ & $33(27 \%)$ & $69(55 \%)$ & $23(18 \%)$ & 125 \\
\hline $51-$ über 60 & $23(33 \%)$ & $28(47 \%)$ & $15(20 \%)$ & 66 \\
\hline
\end{tabular}

In Bezug auf H5 zeigt sich, dass Frauen seltener FÜ-Karrieren wählten und häufiger eine PROJ-Karriere einschlugen als männliche Ingenieure (s. Tab.6). Für die zukünftige Karriereplanung wünschen sich Frauen dagegen genauso häufig wie Männer das Einschlagen einer FÜ-Karriere $(58 \% \mathrm{~m}$ vs. $56 \% \mathrm{w}$ ). Sie bevorzugen stärker als die männlichen Ingenieure eine PROJKarriere $(19 \% \mathrm{~m}$ vs. $26 \% \mathrm{w})$, während sich diese häufiger auf die FAKarriere fokussieren $(23 \% \mathrm{~m}$ vs. $17 \% \mathrm{w})$. Während im Bereich der angestrebten Karriere kein signifikanter Zusammenhang zwischen Frauen und Männern feststellbar ist $(\chi 2=5,19, p>0,158)$, sind die beschriebenen Unterschiede bei den eingeschlagenen Karrieren signifikant $(\chi 2=9,10, p>0,05)$.

Tabelle 6: Gewählter und in Zukunft geplanter Karriereweg von weiblichen und männlichen Ingenieuren

\begin{tabular}{|l|c|c|l|c|c|}
\hline Gewählte Karriere & Männlich & Weiblich & Angestrebte Karriere & Männlich & Weiblich \\
\hline FA-Karriere & $170(37 \%)$ & $22(39 \%)$ & FA-Karriere & $102(23 \%)$ & $10(18 \%)$ \\
\hline FÜ-Karriere & $158(35 \%)$ & $16(28 \%)$ & FÜ-Karriere & $262(58 \%)$ & $32(56 \%)$ \\
\hline PROJ-Karriere & $125(28 \%)$ & $19(33 \%)$ & PROJ-Karriere & $87(19 \%)$ & $15(26 \%)$ \\
\hline Gesamt & 453 & 57 & Gesamt & 451 & 57 \\
\hline
\end{tabular}




\section{Diskussion}

Die vorliegende Untersuchung konnte interessante Entwicklungen in der Karriereplanung von Ingenieuren aufdecken und zeigt insbesondere den Handlungsbedarf in Bezug auf eine stärkere Etablierung alternativer Karrierewege.

H 1 wurde auf Basis der vorliegenden Datenlage bestätigt. Ingenieure, die eine Führungslaufbahn eingeschlagen haben, sind mit ihrer momentanen beruflichen Situation zufriedener und schätzen ihre zukünftigen Karrierechancen positiver ein als Ingenieure der FA- oder PROJ-Karriere. Wollen Unternehmen unterschiedliche Potenziale optimal nutzen, so ist $\mathrm{zu}$ empfehlen, die FÜ- oder PROJ-Laufbahn zu institutionalisieren und mit entsprechenden Anreizsystemen auszustatten, um attraktive Karrierealternativen zu schaffen. Mangelnde Aufstiegsmöglichkeiten und Karriereperspektiven können zu Demotivation und der Abwanderung von leistungsfähigen Ingenieuren führen. Die gleichwertige Etablierung alternativer Karrierewege ist somit eine notwendige Voraussetzung um Ingenieure an Unternehmen zu binden.

H 2 (ein zentraler Grund für den Arbeitsplatzwechsel von Ingenieuren ist ein Gefühl der Unterforderung), konnte bestätigt werden. Es zeigte sich, dass eine Unterforderung zu einer Verstärkung der Wechselbereitschaft führt, vor allem in der Gruppe der Führungskräfte. Das Ergebnis liefert Evidenz für die oben ausgeführte Argumentation, dass unterforderte Mitarbeiter den Eindruck haben, dass ihre hohen Qualifikationen auf keine ausreichenden „Gegenleistungen" der Unternehmung in Form fordernder Tätigkeiten oder attraktiver Vergütung stoßen. Zudem ist ein Wechsel in der Regel mit zusätzlichen Anstrengungen verbunden. Es ist anzunehmen, dass Organisationsmitglieder, die sich bereits überfordert fühlen, sich eher nicht in der Lage sehen, diesen zusätzlichen Aufwand auf sich zu nehmen. Unternehmen stehen vor der Herausforderung mit Hilfe von strukturierten Mitarbeitergesprächen sowie entsprechenden Leistungs- und Kompetenzbeurteilungen aufzudecken, inwieweit sich Organisationsmitglieder unterfordert fühlen. Auf diese Weise können die Potenziale dieser Akteursgruppen genutzt und ein Organisationswechsel verhindert werden.

In Bezug auf H 3 konnte bestätigt werden, dass Ingenieure arbeitsplatznahen WB-Formen eine höhere Bedeutung als externen WB-Angeboten zumessen. Eine Erklärung für den hohen Stellenwert des learning by doing bei Ingenieuren könnte darin liegen, dass sie selbst bereits die mit WB verbundenen Risiken und Chancen erfahren haben (z.B. die Transferproblematik, also die mangelnde Anwendungsmöglichkeiten von erworbenen Kompetenzen in die Praxis). Praktische Implikationen ergeben sich hier vor allem für die Anbieter von externen WB-Maßnahmen (z.B. Hochschulen, Universitäten) für diese Zielgruppe, die vor der Herausforderung stehen, diese für sich zu gewinnen 
und an sich zu binden. Die Ergebnisse der vorliegenden Studie weisen auf die Gefahr hin, dass WB-Einrichtungen an dem Bedarf sowie der Lebens- und Arbeitssituation von Ingenieuren ,vorbei“ WB-Angebote entwickeln. Ausgehend von den Erkenntnissen dieser Studie sollten Hochschulen die WB-Form des „Learning by doing“ in ihr WB-Angebot integrieren sowie mit Inhalten und methodisch-didaktischen Konzepten auf Hochschulniveau verknüpfen.

In Bezug auf $\mathrm{H} 4$ zeigt sich, dass insbesondere die FÜ-Karriere von jüngeren Ingenieuren häufiger als zukünftiger Karriereweg angestrebt wird. Ein solch eindeutiger Trend ist bei den PROJ-Karrieren nicht zu erkennen (keine Bestätigung von H4). Es überrascht, dass die jüngeren Ingenieure dem ,alten" Karrieremuster der FÜ-Karriere verhaftet sind. Ein Grund mag darin liegen, dass die jungen Ingenieure erkennen, dass Unterstützungs- und Fördermaßnahmen von Seiten der Unternehmen und der WB-Anbieter für FÜ-Karrieren im Vergleich zu den anderen Karrieren wie FA- und PROJKarriere am umfangreichsten entwickelt sind (s. H1). Die Entwicklung der Karrierewünsche in Abhängigkeit vom Alter kann auch so gedeutet werden, dass ein großer Teil der Ingenieure zu Beginn ihrer Karriere den Wunsch nach einer FÜ-Karriere haben, ihnen es aber aufgrund von begrenzt vorhandenen Stellen an Führungspositionen nicht gelingt, diesen zu realisieren, sodass sie diesen Wunsch im Laufe der Zeit reduzieren. In diesem Zusammenhang besteht die Gefahr, dass es bei den betroffenen Personengruppen im Laufe ihres Erwerbslebens zu enttäuschten Erwartungen kommt, die sich negativ auf Arbeitsverhalten und -leistung auswirken können. Eine Etablierung der PROJ-Karriere als alternative Leitkarriere würde der Problematik entgegenwirken und die Verknüpfung sowie Weiterentwicklung von Fach- und Führungskompetenzen ermöglichen.

Zusammenhängend mit $\mathrm{H} 5$ bestätigt sich, dass Ingenieurinnen häufiger als Ingenieure eine PROJ-Karriere wählten und für die Zukunft anstreben. Allerdings liegt der Prozentsatz deutlich unter dem der für die Zukunft angestrebten FÜ-Karriere. Die große Diskrepanz zwischen eingeschlagener FÜKarriere und für die Zukunft gewünschter FÜ-Karriere vor allem bei Ingenieurinnen weist darauf hin, dass Unternehmen ein deutliches Potenzial an weiblichen Mitarbeiterinnen nicht ausreichend in FÜ-Karrieren integrieren. Gerade vor dem Hintergrund der Einführung von Quotenregelungen zur Erhöhung des Frauenanteils in FÜ-Positionen sind die vorliegenden Ergebnisse tatsächlich so zu deuten, dass Ingenieurinnen an eine „gläserne Decke“ zu stoßen scheinen.

Die Grenzen der vorliegenden empirischen Untersuchung beziehen sich auf folgende Punkte: (1) Verschiedene Dimensionen, wie z.B. Zufriedenheit, Überforderung oder Zukunftsperspektiven, hätten durch mehrere Items abgefragt werden können. (2) Die Aussagekraft bezüglich des Gender-Aspekts sollte aufgrund des niedrigen Anteils weiblicher Befragter durch weitere Untersuchungen gestützt werden. (3) Domänenspezifische Artefakte können die 
Untersuchungsergebnisse verzerrt haben. Es kann beispielsweise vermutet werden, dass die Spezifizierungsrichtung der Ingenieure (z.B. Wirtschafts-, Vermessungs- oder Bauingenieur) einen Einfluss auf die Karrierechancen in den drei unterschiedlichen Laufbahnen (FÜ, FA, PR) hat. Zudem ist denkbar, dass Ingenieure sich je nach Ausbildungsstand (Fachhochschule, Universität, Promotion) in ihren Weiterbildungswünschen unterscheiden.

\section{Berufs- und wirtschaftspädagogischer Ausblick}

Die vorausgegangenen Ausführungen haben verdeutlicht, dass die einseitige Orientierung der Erwerbsbiographien der Ingenieure an der FÜ-Karriere an Grenzen stößt und letztlich wenig hilfreich ist. Der Ausbau der Unterstützungsmaßnahmen für die Weiterentwicklung und Etablierung von FA- und PROJ-Karrieren ohne die Vernachlässigung der FÜ-Karrieren erscheint dringend geboten. In diesem Zusammenhang sind alle Akteure herausgefordert, vorausgesetzt die Potentiale der Ingenieure sollen genutzt werden: Aus- und WB-Anbieter, Unternehmen, Personalabteilungen, Führungskräfte in ihrer Rolle als Personalentwickler sowie die Ingenieure und Ingenieurinnen selbst. Ausgehend von den aufgezeigten Unterschieden zwischen den Ingenieuren, die sich für eine FÜ-, FA- oder PROJ-Karriere entschieden haben, besteht die Möglichkeit die WB von Ingenieuren in Zukunft noch zielgruppenspezifischer auszurichten und die WB-Formen adäquater einsetzen zu können. Es stellt sich beispielsweise die Frage, wie learning-by-doing-Strategien verbessert werden können und in welcher Form Ingenieure von Dritten unterstützt werden können.

Unter der Voraussetzung, dass Ingenieure in hohem Maße in innovative Entwicklungsprozesse eingebunden sind und Innovationen in vielen Fällen auf der Verknüpfung von zwei oder mehreren Fachgebieten basieren, überrascht es nicht, wenn insbesondere FA-Karriereingenieure sich weitere Fachkenntnisse aneignen. Sie benötigen diese, um aktuelles Fachwissen zu verknüpfen und für innovative Ideen zu nutzen. Wenn im Zusammenhang mit der WB von Ingenieuren primär die Programme zur Soft-SkillsEntwicklung weiter ausgebaut und überproportional von den Unternehmen sowie WB-Anbietern offeriert werden, dann führt dies letztlich zu einer Benachteiligung von FA-Karriereingenieuren. 


\section{Literatur}

Adams, J.S. (1965). Inequity in social exchange. In L. Berkowitz (Ed.), Advances in experimental social psychology (Vol. 2, pp. 267-299). New York:.

Corria-Simpson, N.-D. A., Langer, B. R. \& Handor, P. (2008). Project Controls: a Roadmap to a Choice Career. AACE International Transactions, pp. 1-10.

Gerstenfeld, A.,\& Rosica, G. (1970). Why engineers transfer: Survey pinpoints reasons for job changes. Business Horizons, 13(2), 43.

Hermann, A. (2004): Karrieremuster im Management. Wiesbaden.

Hodgson, D., Paton, S. \& Cicmil, S. (2011). Great expectations and hard times: The paradoxical experience of the engineer as project manager. Journal of Project Management, doi: 10.1016/j.ijproman.2011.01.005

Lam, A. (2000). Tacit Knowledge, Organizational Learning and Societal Institutions: An Integrated Framework. Organization Studies, 21(3), S. 487-513.

Lienert, A. (2010): Neue Karrieren bei Ingenieurinnen und Ingenieuren? Bochum (unver. MA-arbeit)

Majer, C. \& Mayrhofer (2007). Konsequent Karriere machen. Personal 11/2007, S. 36-39.

March, J. G. \& Simon, H. A. (1958). Organizations. New York, John Wiley.

Nitsche, S. (2011). Kooperation kontra Konkurrenz. Medieninformation Nr. 219/2011. http:/www.pressestelle.tu-berlin.de/medieninformationen/2011/juli_2011/medien information_nr_2192011/ (Abruf am 10.01.2012).

Nonaka, I. \& Takeuchi, H.: Die Organisation des Wissens. Frankfurt: Campus.

Skiba, M., \& Rosenberg, S. (2011). The Disutility of Equity Theory in Contemporary Management Practice. Journal Of Business \& Economic Studies, 17(2), pp. 1-19.

Strunk, G. (2009): Die Komplexitätshypothese der Karriereforschung. Frankfurt 2009. Thom, N. (2008). Moderne Personalentwicklung. Wiesbaden. 



\title{
Diffusion von Technologien in die Facharbeit und deren Konsequenzen für die Qualifizierung am Beispiel des „Internet der Dinge“
}

\author{
Lars Windelband; Georg Spöttl
}

\section{Einleitung}

In zahlreichen Feldern der Arbeitswelt haben sich in den letzten Jahren durch die Einführung von IT-Systemen fundamentale Veränderungen vollzogen. Vor allem durch die Vernetzung mittels IT-Anwendungen entstanden viele Möglichkeiten, komplexe Systeme und Prozesse zu überwachen und immer mehr ohne menschliches Zutun zu steuern. Weyer (2007, S. 35) stellt dazu fest: ,ein besonderes Merkmal der jüngsten Entwicklung ist zudem das scheinbar unaufhörliche Vordringen autonomer technischer Systeme, die immer mehr zu Mitspielern in derartigen Netzwerken geworden sind“. Konsequenz aus dieser Entwicklung ist das Entstehen „hybrider Konstellationen, die von menschlichen Akteuren und (teil)autonomen Maschinen“ (ebd.) durchdrungen sind. Wie nachstehend gezeigt wird, nimmt die Verselbstständigung von IT-Systemen Schritt um Schritt zu, so dass die Rolle der menschlichen Akteure in den Hintergrund gedrängt wird. Die Auslöser sind neue High-Tech-Entwicklungen im Bereich RFID (Radio-Frequency Identification) und dem „Internet der Dinge“"1.

Was bedeutet dies konkret für die betrieblichen Prozesse? Ein Paket wäre in einem logistischen Prozess nicht mehr nur einfach ein Fördergut, welches von A nach B bewegt wird. Es nimmt stattdessen direkten Einfluss auf seinen eigenen Weg. Ausgehend von dem Ansatz des „Internet der Dinge“ wird das Paket mit einer „Intelligenz“ bestückt. Es selbst erkennt z. B. durch den Einsatz von RFID-Labels das Ziel im logistischen System.

1 Der Begriff „Internet der Dinge“ wurde erstmals im Jahre 1999 vom Massachusetts Institute of Technology (MIT) geprägt. Definiert wurde der Begriff als ,[...] ein informationstechnisch vernetztes System autonom interagierender Gegenstände und Prozesse, die sich durch eine zunehmende Selbstorganisation charakterisieren und zu einer wachsenden Verschmelzung physischer Dinge mit der digitalen Welt des Internets führen" (Brand et al. 2009, S. 107). Das Institut für Materialfluss und Logistik (IML) versteht für den Logistikbereich unter dem „Internet der Dinge“ den autonomen, selbstgesteuerten Transport logistischer Objekte vom Absender zum Adressaten (vgl. Ten Hompel 2005). 
Diejenigen Fördermittel, die im logistischen Prozess integriert sind, sind mit RFID-Readern ${ }^{2}$ ausgerüstet und können somit das Label des Paketes auslesen. Die Information, die auf den RFID-Label gespeichert ist, kann nun zur Steuerung des Logistikprozesses genutzt werden. Die Entscheidung über den genauen Weg des Paketes trifft nun nicht mehr ein zentrales Leitsystem oder der Mensch, sondern das Transportmittel in Kooperation mit dem Paket.

Durch diese autonome Steuerung der betrieblichen Prozesse ist eine neue Stufe der technischen Entwicklung erreicht. Diese wirkt sich entscheidend auf die sozialen und wirtschaftlichen Organisationsformen und die Organisation der Arbeit aus. Im Gegensatz zu Maschinen, die Aufgaben nach vom Menschen bestimmten und gesteuerten Prozessen erledigen, vermögen die vernetzten Systeme im „Internet der Dinge“ Entscheidungen selbständig zu treffen. Mit der Umsetzung des „Internet der Dinge“ ist daher in der MenschMaschine-Interaktion ein neues Niveau der Arbeitsteilung erreicht.

\section{Entwicklungstand des „Internet der Dinge“}

Als potenzielle Anwendungsmöglichkeiten des „Internet der Dinge“ im Bereich der Logistik werden in der Literatur die Identifikationstechnologien gesehen (Barcode, RFID), die digitalen Produktgedächtnisse ${ }^{3}$, die intelligente Vernetzung von Produkten und das selbständige Handeln mittels spezieller Softwareagenten- und Assistentensysteme. Weitere Anwendungsfelder sind logistische Steuerungen und Trackingsysteme und der selbstorganisierte Transport logistischer Objekte durch inner- und außerbetriebliche Transportnetze (vgl. Ten Hompel/Bullinger 2007).

Die technologische Grundlage des „Internet der Dinge“" (vgl. Dworschak et al. 2011, S. 2) bildet die Ausstattung von Gegenständen, Räumen und Maschinen mit verschiedenen Technologien zur Umgebungswahrnehmung, Datenspeicherung, Kommunikation und zum autonomen Handeln. Hierzu gehört immer mehr die Radiofrequenzidentifikation (RFID). RFID-Systeme können Daten berührungslos und ohne Sichtkontakt auslesen. Sie bestehen

2 RFID-Reader: Spezielles elektronisches Gerät, das elektromagnetische Wellen einer spezifischen Frequenz und Reichweite aussenden und empfangen kann, um den Inhalt von RFID-TAGs auszulesen. (Zahn 2007, S. 31ff.).

RFID-TAG: Sehr kleiner Mikrochip mit integrierter Antenne, der bei Empfang von spezifischen Radiowellen in ihm abgelegte Daten per Radiowellen aussendet.

3 Hier werden Objekte mit „Smart Label“ ausgestattet, die RFID (Radio Frequency Identification) mit eigener Stromversorgung und unterschiedlicher Sensorik kombinieren. Dadurch wird es möglich, dass die Produkte selbst Daten aus ihrer Umgebung sammeln. So können Warenzustand, Frischegrad, Lagertemperatur, Herkunft etc. überwacht und jederzeit ausgelesen werden (vgl. Brand et al. 2009, S. 106f.) 
aus einem Lesegerät und Transponder bzw. „Tag“, d.h. einem Chip mit Antenne. Dieser ermöglicht eine eindeutige Identifikation des Gegenstandes, auf dem er angebracht ist. Damit ist es möglich, den genauen Weg der einzelnen Produkte nachzuvollziehen. RFID-Systeme werden mit Sensorik kombiniert, die in Produkte eingebettet sein kann. Diese Sensordaten können mittels RFID automatisch aktualisiert werden. Aufgrund gestiegener Anforderungen bezüglich Beweglichkeit und Mobilität geht die Entwicklung der RFIDSysteme und Sensorik zu drahtlosen Systemen, für die eine funkbasierte Informationsübertragung entscheidend ist.

Nach Meinung vieler Experten wird das „Internet der Dinge“ zu einer erheblichen Optimierung der logistischen Prozesse führen. Durch aktive Sensorik in Transportträgern kann z. B. eine bislang unbekannte engmaschige Prozessüberwachung realisiert werden, die das Aufspüren von Fehlern und Fehlerquellen deutlich vereinfacht. Logistische Abläufe können damit nachhaltig besser organisiert und letztlich kostengünstiger optimiert werden. Beispiele für die Anwendung sind Überwachungslösungen für Waren, Fahrzeuge und Transportbehälter. Als Nebeneffekt dieser vollständigen Prozessüberwachung und -dokumentation wäre auch der Kunde jederzeit in der Lage, Informationen über den Status innerhalb der Logistikkette zu seinen transportierten Produkten abzurufen. Dies kann zu einem Wettbewerbsvorteil führen, wenn kurze Laufzeiten angestrebt werden oder bei Logistikketten die vielen unterschiedlichen, oft kritischen Durchlaufstationen (z.B. Ländergrenzen, Zoll) eindeutig identifizierbar und nachverfolgbar sind.

Das „Internet der Dinge“ bietet jedenfalls zahlreiche Möglichkeiten, um die Effizienz und Effektivität logistischer Prozesse zu verbessern. Bei diesen technologiebasierten Entwicklungen dominiert eine technikzentrierte Automatisierungsstrategie, also das Bestreben, fehlerfreie technische Systeme zu entwickeln und zu implementieren und den Menschen als mögliche Störquelle auszuschalten. Die Symmetrie zwischen Mensch und Maschine ist dabei ein entscheidender Faktor und spielt bisher in der Entwicklung der einzelnen Technologien und Produkte eine eher untergeordnete oder gar keine Rolle.

\section{Fragestellung zur Diffusion des „Internet der Dinge“}

Im vorliegenden Artikel soll vor allem die Diffusion des „Internet der Dinge“ in die realen Arbeitsabläufe auf der Facharbeitsebene näher betrachtet werden. Grundlage dafür ist eine Früherkennungsstudie, die im Auftrag des Bundesministeriums für Bildung und Forschung (BMBF) durchgeführt wurde. Dabei wurde u.a. folgende Fragestellung untersucht: Inwieweit wird die Facharbeit im Logistikbereich bereits mit dem „Internet der Dinge“ konfrontiert und welche Folgen hat dieses für deren Organisation? 
Denn bisher existierten keine konkreten Informationen über den Umsetzungsgrad des „Internet der Dinge“ in der Unternehmens- und Arbeitswelt und damit auch keine Informationen $\mathrm{zu}$ veränderten Qualifikationsanforderungen der Beschäftigten. Die durchgeführte Studie soll erste Auswirkungen dieser Zukunftstechnologie auf die Facharbeit beschreiben. Sie ist eingebettet in die Früherkennungsinitiative des vom BMBF geförderten Forschungsnetzes FreQueNz ${ }^{4}$. Hauptziel der Früherkennungsforschung ist es, künftige, neue oder veränderte Qualifikationsanforderungen in der Berufs- und Arbeitswelt möglichst früh zu ermitteln und schnell in den Prozess der Berufsbildungsgestaltung einzubringen.

Insgesamt wurden drei Studien mit dem Schwerpunkt „Internet der Dinge" in den Anwendungsfeldern: Logistik ${ }^{5}$, Industrielle Produktion und Smart House gefördert, um die konkreten Auswirkungen der technologischen Entwicklungen in der Arbeitswelt und der Facharbeit zu erschließen.

Neben der Betrachtung der technischen Entwicklung des „Internet der Dinge" sowohl in Forschung als auch in der Praxis der Distributionslogistik soll eine Auseinandersetzung darüber geführt werden, in welcher Form Facharbeit betroffen ist, um Aussagen treffen zu können, inwieweit sich mögliche Geschäftsprozesse und Arbeitsabläufe bis hin zu Arbeitsaufgaben innerhalb der Unternehmen verändert haben. Aus den Erkenntnissen werden Schlüsse für Qualifizierungsnotwendigkeiten gezogen.

\section{Forschungsdesign der Früherkennungsstudie zum "Internet der Dinge“}

Die Früherkennungsstudie diente der Identifikation der Veränderungen der Qualifikationsanforderungen innerhalb von ausgewählten Berufen und innerhalb der betrieblichen Arbeitswelt für einen Zukunftszeitraum von drei bis fünf Jahren. Untersuchungsgegenstand war das „Internet der Dinge“ in der Logistik. Als Ergebnis sollten Informationen bereitgestellt werden, um eine systematische Beobachtung der Qualifikationsentwicklung zu ermöglichen. Im Mittelpunkt steht dabei die Untersuchung neuer Qualifikationen in der Frühphase ihrer Entstehung und die Beurteilung ihrer zukünftigen Entwicklung (vgl. Bullinger/Tombeil 2000, S. 23).

4 FreQueNz ist ein vom Bundesministerium für Bildung und Forschung gefördertes Forschungsnetz in Deutschland, in dem verschiedene Institute mit ihrer Projektarbeit zur Früherkennung von Qualifikationserfordernissen beitragen.

5 Die beiden Autoren leiteten die Untersuchungen für den Bereich Logistik im Rahmen der FreQueNz-Früherkennungsstudie (vgl. Windelband et al. 2011). 
Für die Beantwortung der Forschungsfrage wurden drei Untersuchungsschritte $^{6}$ gewählt, die qualitative berufswissenschaftliche Früherkennungsinstrumente (vgl. Windelband 2006, Windelband/Spöttl 2003) nutzen:

1. Schritt: Analyse des aktuellen Standes der Durchdringung der Distributionslogistik mit dem „Internet der Dinge“ in den Sektoren Lebensmittel und Automobilindustrie.

Auswahl der Sektoren: Die beiden Sektoren wurden in einer Vorstudie ausgewählt. Identifiziert wurden dabei Praxisgebiete, in denen das „Internet der Dinge" bereits angewandt wird.

2. Schritt: Analyse von innovativen Unternehmen und Forschungsprojekten zur Implementierung und Umsetzung des „Internet der Dinge“ in der Facharbeit?

Auswahl von Unternehmen für Fallstudien: Es wurden Unternehmen gesucht, die Technologien zum „Internet der Dinge“ einsetzten oder pilothaft erprobten und bereit waren Fallstudien zuzulassen. Mittels der Fallstudien in den Forschungseinrichtungen sollten innovative Umsetzungsprojekte erschlossen ${ }^{8}$ werden.

3. Schritt: Vertiefende qualitative Analysen (Interviews mit Fachexperten) zur Identifikation von Entwicklungsrichtungen und Verbreitung des „Internet der Dinge" in der Facharbeit und möglicher Konsequenzen für die Qualifizierung.

Auswahl von Experten: Neben der Voraussetzung, Experte für das „Internet der Dinge" zu sein, wurden bei der Auswahl folgende weitere Parameter zugrunde gelegt: hoher Praxisbezug, Mitarbeit an zukunftsweisenden Projekten sowie Schwerpunkt in einem der beiden Anwendungsbereiche Lebensmittel- und Automobilindustrie.

Schritt 1 wurde in einer Vorstudie bewältigt und dabei wurden zu untersuchende Unternehmen und Forschungsprojekte und deren Forschungseinrichtungen ausgewählt.

Mittels Schritt 2 sollte der technologische Entwicklungstand des „Internet der Dinge“ im Bereich von Unternehmen und Wissenschaft/ Forschung erschlossen werden. Dazu wurden aktuelle Projekte analysiert und Expertengespräche mit Forschungsvertretern durchgeführt, um herauszufinden, wie

6 Das Instrument des Zukunftsexperten-Workshops wurde als viertes Instrument für mögliche neue und veränderte Qualifikationserfordernisse eingesetzt. Diese Fragestellung wird in diesem Beitrag nicht genauer diskutiert.

7 Untersucht wurden sechs Betriebe und vier Forschungsinstitute.

8 Ein Fall in den Anwendungsbereichen Lebensmittel- oder Automobilindustrie wird repräsentiert durch ein Forschungsprojekt, welches Entwicklungs- und Umsetzungsziele zum „Internet der Dinge“ im Bereich der Distributionslogistik verfolgt. 
weit die technologische Entwicklung fortgeschritten ist und welche Probleme bei der Implementierung in die Praxis bestehen. Ein Instrument zur Feststellung der „Diffusionstiefe“ des „Internet der Dinge“ wurde entwickelt. Dieses beinhaltet sechs charakteristische Merkmale (vgl. Tabelle 1), mit deren Hilfe die Diffusionstiefe dargelegt werden sollte. Die Merkmale im Einzelnen: Technologie, Energieversorgung, Konnektivität, Informationsverarbeitung, Aggregationsebene und Verortung der Intelligenz. Dahinter verbirgt sich jeweils:

- Bei der „Technologie” beschreibt die Stufe 1 eine Auto-ID (Identifikation), zum Beispiel ein RFID- oder Barcodesysteme. Stufe 2 beschreibt den Einsatz von Sensorik. In Stufe 3 werden schließlich eingebettete Systeme verwandt, das heißt, dass Entscheidungsfindungskomponenten verbaut werden.

- Das Merkmal der „Energieversorgung“ wird in der Stufe 1 über Induktion bewerkstelligt, wie das Auslesen und Beschreiben von passiven RFID-Transpondern. Die Stufe 2 zeichnet sich durch Systeme aus, die durch einen Akkumulator mit Energie versorgt werden (aktive RFIDTransponder). Systeme der Stufe 3 haben eine autarke Energieversorgung.

- Das Merkmal „Konnektivität“ beschreibt in der Stufe 1 das manuelle Auslesen von Informationen, das System kommuniziert also rein passiv. In Stufe 2 kommuniziert das System bei Bedarf, es könnte sich zum Beispiel um eine Meldung an ein Steuerungszentrum oder einen Supervisor bei einem bestimmten Ereignis, wie des Überschreitens eines Messwertes, handeln. Stufe 3-Systeme kommunizieren durchgehend mit anderen Systemen und sind ständig ,online“.

- Das Merkmal „Informationsverarbeitung“ gibt an, wie das Objekt mit den Informationen umgeht. Bei Stufe 1 werden Informationen aufgenommen und gespeichert, aber nicht weiter verarbeitet (z.B. Temperaturlogger). In Stufe 2 können Informationen an andere Instanzen weitergereicht werden, werden aber vom Objekt selber nicht verarbeitet. Dies sind z. B. Telematik-Systeme, die bei bestimmten Ereignissen eine Statusmeldung abschicken können. In Stufe 3 ist das Objekt in der Lage, eine Entscheidung zu treffen. Dies gilt für eingebettete Systeme.

- Die „Aggregationsebene“ einer Technologie: In der Stufe 1 befindet sich die Technologie auf der Verpackung, zum Beispiel einem Karton oder Container. Stufe 2 beschreibt eine Technologie auf Objektebene (Ebene des fertigen Endproduktes). Bei Fahrzeugen würde beispielsweise diese Technologie pro Fahrzeug eingesetzt. Stufe 3 Systeme werden auf Komponentenebene eingesetzt, in Fahrzeugen zum Beispiel auf einem Sitz. 
- Bei der „Verortung der Intelligenz“ sind in der ersten Stufe die Intelligenten Systeme in einem Netzwerk verteilt (mögliches Einsatzszenario Sensornetzwerk). Stufe 2 wird charakterisiert durch Intelligenz auf Objektebene, also auf dem Endprodukt und Stufe 3 beschreibt eine verteilte Verortung der Intelligenz, d.h. verschiedene Teilsysteme werden auf verschiedenen Ebenen eingesetzt, sind aber zu einem System verbunden.

Tabelle 1: Ausprägung des „Internets der Dinge“ für den Bereich der Logistik

\begin{tabular}{|c|c|c|c|}
\hline Stufe & 1 & 2 & 3 \\
\hline Technologie & Auto-ID & Sensorik & $\begin{array}{c}\text { Eingebettestes } \\
\text { System }\end{array}$ \\
\hline $\begin{array}{l}\text { Energie- } \\
\text { versorgung }\end{array}$ & Induktion & Akku & Autark \\
\hline Konnektivität & $\begin{array}{l}\text { Manuelles } \\
\text { Auslesen }\end{array}$ & Bei Bedarf & Durchgehend \\
\hline $\begin{array}{l}\text { Informations- } \\
\text { verarbeitung }\end{array}$ & Speicherung & Mitteilung & $\begin{array}{l}\text { Entscheidungs- } \\
\text { findung }\end{array}$ \\
\hline $\begin{array}{l}\text { Aggregations- } \\
\text { ebene }\end{array}$ & $\begin{array}{l}\text { Verpackungs- } \\
\text { ebene }\end{array}$ & Objektebene & Komponente \\
\hline $\begin{array}{l}\text { Verortung der } \\
\text { Intelligenz }\end{array}$ & Netzwerk & Objekt & Verteilt \\
\hline
\end{tabular}

Gleichzeitig wurden über die Experten Unternehmen identifiziert, die Technologien zum „Internet der Dinge“ in den Anwendungsbereichen einsetzen. Diese wurden noch im Schritt 2 mittels des Instruments der Fallstudien genauer untersucht und die Ergebnisse wurden in das Instrumentarium zur Bewertung der Ausprägungsstufen eingeordnet.

Die Ergebnisse aus den ersten beiden Schritten wurden im dritten Schritt mittels qualitativer Interviews zur Erarbeitung erster Entwicklungsrichtungen verdichtet. Zielgruppen waren ausgewählten Unternehmensvertreter und Experten der Branche.

Im vierten Schritt der Studie (Zukunfts-Experten-Workshops) wurden aus den Ergebnissen der vorangegangenen Untersuchungsschritte konkrete Szenarien zu möglichen neuen und veränderten Qualifikationserfordernissen durch die Umsetzung des „Internet der Dinge“ entwickelt. Die Konzentration auf Zukunftstechnologien zum „Internet der Dinge“, die noch am Anfang der Implementierung in der Praxis stehen, ermöglicht es, frühzeitig Schlussfolgerungen zu möglichen Konsequenzen für die Beschäftigten $\mathrm{zu}$ ziehen. Damit kann gezielt Auskunft über mögliche Zukunftsentwicklungen für die nächsten fünf Jahre gegeben und erste Handlungsoptionen für die berufliche 
Bildung können aufgezeigt werden, um den technologischen Entwicklungen nicht ständig ,hinterherrennen“ zu müssen.

\section{Untersuchungsergebnisse}

Obwohl zu erkennen war, dass in den Unternehmen eine zunehmende Auseinandersetzung mit dem „Internet der Dinge“ (IdD) stattfindet, war der Diffusionsgrad meist relativ gering. Die Auswertung der Befragungen und Fallstudien zeigten, dass kaum ein Unternehmen die zweite oder gar dritte Stufe erreicht hat. Vor allem kommunizieren die Objekte bisher nicht eigenständig miteinander und nehmen keinen direkten Einfluss auf die Warenströme. In den untersuchten Praxisfeldern der Unternehmen, also den konkreten Einsatzfeldern des IdD, konnte nur eine geringere Diffusion der IdD-Technologien festgestellt werden. Diese erreichten meist nur Stufe 1 nach Tabelle 1.

Trotz dieser generellen Erkenntnisse muss für den Bereich der Automobillogistik festgestellt werden, dass Unternehmen identifiziert werden konnten, die die zur Umsetzung des „Internet der Dinge“ benötigten Basistechnologien bereits verfügbar hatten. Solche Unternehmen arbeiten zumindest mit Barcodes bzw. setzten RFID ein. Ausgelesen werden die Daten meist manuell mit einem entsprechenden (mobil oder stationär eingesetzten) Barcodereader. Nur bei den Unternehmen mit RFID-Technologie erfolgt das Auslesen der Daten automatisch. Hier waren auch die Objekte mit einem Datenspeicher versehen, womit eine Vernetzung, wie es das „Internet der Dinge" vorsieht, möglich wäre. Meist ist es ein betriebspezifisches IT-System, das speziell auf die Belange des Unternehmens zugeschnitten ist und in den meisten Fällen eigens für den vorgesehenen Zweck entwickelt wurde. Kein Unternehmen setzt die Technologie betriebsübergreifend ein oder überlässt Entscheidungen einem Algorithmus, der die benötigten Daten von integrierten Objekten erhält. Die Daten werden für die Optimierung der Logistikkette (oft nur bis zum Hoftor) verwendet. Mit ihrer Hilfe werden Prozesse überwacht, optimiert und die Produktdurchgänge sowie Warenbestände kontrolliert.

Die Informations- und Kommunikationssysteme werden in vielfältiger Form angewandt. In allen Betrieben unterstützen sie die Mitarbeiter im operativen Bereich. Über das Display werden Arbeitschritte, benötigte Objekte etc. angezeigt und die Bedienung beschränkt sich in der Regel auf einfache Eingaben (Anzahl der Teile, Barcodes etc.) in vordefinierten Feldern.

Im Bereich der Lebensmitteldistribution war der Einsatz der Technik sehr divergent. Alle Unternehmen benutzen heute Telematiksysteme, mit denen die Disponenten und Fahrer über ein Netzwerk in stetiger Verbindung 
sind. Die Computer der Disposition sind mit den an Bord der Fahrzeuge befindlichen PDA oder Notebooks verbunden. Diese Bordcomputer haben meist mehrere Funktionen integriert. Sie werden für die Tourabwicklung eingesetzt, können aber auch für E-Mail-Versendung, als Mobiltelefon oder Navigationsgerät eingesetzt werden. Die komplexen Werkzeuge (Flottenmanagementsysteme), die in der Disposition eingesetzt sind, werden zur Tourplanung, -abwicklung, -überwachung und zur Positionsbestimmung von Transportfahrzeugen und Ware benutzt.

Die Basistechnologien, die für das „Internet der Dinge“ notwendig sind, werden nur am Rande eingesetzt. Eines der drei untersuchten Unternehmen setzt RFID intern ein, um das richtige Waschprogramm für die Transportbehälter zu starten. Ein anderes benutzt Sensorik, um die Temperatur in Kühlcontainern zu überwachen. Bei einem Temperaturanstieg erhält der Disponent eine Nachricht über SMS auf sein Mobiltelefon und muss dann die Entscheidung treffen was mit dem Container geschehen soll. In beiden Betrieben wird vorerst nicht daran gedacht, das System weiter auszubauen und einen selbststeuernden Algorithmus zugrunde zu legen.

Abbildung 1: Ausprägungs- und Diffusionsstufen des „Internets der Dinge“ in den untersuchten Logistikunternehmen

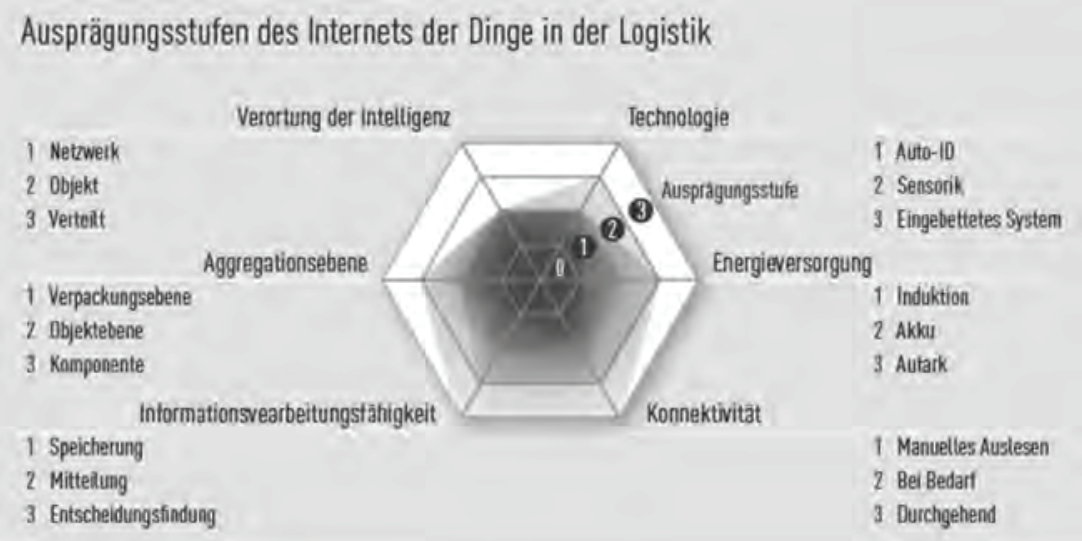

Abbildung 1 zeigt die Merkmalsausprägungen der sechs untersuchten Fallstudienbetriebe hinsichtlich des Technologieeinsatzes in einem Radialdiagramm. Es wird deutlich, dass kaum ein Unternehmen die zweite oder gar die dritte Stufe erreicht hat. Für das Anwendungsfeld der Logistik wird klar, dass die IdD-Technologien bislang vorwiegend betriebs- bzw. unternehmensintern eingesetzt werden („Intranet der Güter“). Als größtes Hindernis bei der Investition in neue Systeme werden auch hier die Kosten gesehen. Eine nega- 
tive Kosten-Nutzen-Einschätzung, eine eingeschränkte Lesbarkeit von RFIDChips in metallischen Umgebungen und Flüssigkeiten sowie auch das Fehlen einheitlicher Standards verhindern eine Investition in neue Technologien zum „Internet der Dinge“.

Es ist nicht auszuschließen, dass in Zukunft weitere Ausprägungsstufen hinzukommen können. Die drei Stufen zeigen, wie weit die Diffusion fortschreiten kann, wenn die Entwicklungen zur Umsetzung der „Vision“ des „Internet der Dinge“ folgen. Das bedeutet jedoch nicht, dass Ausprägungsstufe drei die „,ideale Stufe“ für eine Logistikkette über mehre Unternehmen hinweg verkörpert. Dies ist sehr stark von den logistischen Prozessgegebenheiten abhängig. In der Ausprägungsstufe drei über alle sechs Kriterien würde der Prozess automatisiert und mit einer eigenständigen Entscheidungsfindung ablaufen. Damit kommt diese Stufe dem „Internet der Dinge" mit einem autonomen und selbstgesteuerten Transport sehr nahe, ist jedoch nicht unbedingt für jedes Unternehmen erstrebenswert, geschweige eine Richtung, die dem Menschen noch viele Optionen einräumt.

\section{Konsequenzen der Diffusion des „Internet der Dinge“6 für die Facharbeit und daraus resultierende Qualifikationsanforderungen}

Trotz der bisher geringen Diffusion des IdD in die logistischen Prozesse lassen sich Unternehmen ausmachen, bei denen Abläufe tatsächlich nachhaltig verändert wurden und sich auch die Aufgaben der Mitarbeiter gewandelt haben. Zentrale Zielrichtung ist bei diesen Unternehmen die Automatisierung von Vorgängen und die Digitalisierung von Informationen. Ein TechnologieMix aus digitalisierten Dokumenten, Erfassung von Geopositionen per Telematik, speziellen On-Board-Terminals für die Zustandserfassung und einem integrierten Informationsfluss über das Internet erlaubt diesen Unternehmen, eine effizientere Dienstleistung zu erbringen. Dabei konnten im Wesentlichen zwei Entwicklungsrichtungen identifiziert werden:

1. Zum einen wurde die Technik benutzt, um Prozesse zu automatisieren und die Fehlerhäufigkeit in den Arbeitsprozessen zu reduzieren. Die Aufgaben und das damit verbundene Anforderungsprofil auf Facharbeitsebene haben sich dadurch häufig vereinfacht. Das hatte zur Folge, dass niedriger qualifiziertes Personal zu geringeren Lohnkosten und ohne lange Anlernzeiten schnell eingesetzt werden konnte.

2. Zum anderen wurden Technologien zum „Internet der Dinge“ genutzt, um Arbeitsabläufe zu optimieren. Gleichzeitig wurden dadurch die Aufgabenfelder bei Mitarbeitern verändert. Die durch die Implementierung 
frei werdenden Kapazitäten bei Mitarbeitern wurden in der Folge mittels anderer Aufgaben belegt, was meist zur Folge hatte, dass die Aufgabenfelder umfangreicher und vor allem vielfältiger wurden. In solchen Betrieben wurde in der Regel auf sehr gut ausgebildete Fachkräfte gesetzt und durch Weiterbildung auf die Aufgabenveränderung reagiert.

Während bei der ersten Entwicklungsrichtung vor allem die An- und Ungelernten in den Unternehmen betroffen sind, ist dies bei der zweiten Richtung die Gruppe der Facharbeiter. Die skizzierten Entwicklungsrichtungen führen $\mathrm{zu}$ verschiedenen arbeitsorganisatorischen Lösungen und haben unterschiedliche Auswirkungen auf die in Frage stehenden Fachkräftegruppen. Im Zuge der Studie konnten für letztere Zielgruppe drei verschiedene Anforderungsprofile identifiziert werden ${ }^{9}$ :

- Disponent,

- Fahrer für Nah- oder Fernverkehr sowie

- Logistikplanung.

Zielt die Entwicklungsrichtung auf eine Automatisierung der Prozesse ab, dann übernimmt die Technik Entscheidungen und führt, per programmgesteuerter Abläufe technologisch vordefinierte Arbeitsroutinen durch. Die bedienungs- und operativen Aufgaben der Fachkräfte vereinfachen sich in diesem Falle, mit Ausnahme von Störungen und anderen Problemen mit dem System selbst. Letztere müssen dagegen von hochqualifizierten Fachkräften gelöst werden. In den untersuchten Unternehmen gab es keine einheitliche oder eindeutige Vorgehensweise zur Gestaltung der Arbeitsorganisation, um diese konträren Aufgaben zu organisieren.

Bei der Entwicklungsrichtung, die Arbeitsabläufe mit Hilfe des „Internet der Dinge“ zu optimieren, ging es in den Unternehmen immer auch darum, die Stärken der Mitarbeiter zu berücksichtigen. Das hatte zur Folge, dass die Entscheidungsfindungsprozesse nicht dem System, sondern dem Menschen überlassen wurden. Die Mitarbeiter bekamen in der Regel unterstützende Informationen zur Prozessoptimierung, konnten jedoch die Prozesse mit gestalten. Die Fachkräfte benötigen dafür ein hohes prozessspezifisches Wissen, um bei Problemen einzugreifen oder Entscheidungen treffen zu können. Fachkräfte haben bei dieser Entwicklungsrichtung die Chance, die Technologien als Assistenzsysteme zu nutzen.

Bei einer Entwicklung zu mehr Automatisierung, oder besser einer weiteren Teilautomatisierung, wie sie zurzeit realistisch erscheint, muss in Problemsituationen oder zur Anpassung in den Prozess eingegriffen werden. Die Fachkräfte, die im alltäglichen Arbeitsprozess mit den entsprechenden Tech-

9 Diese drei Anforderungsprofile sind ausführlich im Abschlussbericht der Studie beschrieben (Windelband et al. 2011, S. 66 ff.). 
nologien arbeiten, sollen zwar einen ordnungsgemäßen Prozess gewährleisten, haben aber nur wenig Einfluss auf die Prozessabläufe, da sie die ITSysteme nicht verändern können. In den meisten Unternehmen werden die entsprechenden IT-Entwicklungen von eigenen IT-Spezialisten umgesetzt. Es wird in Zukunft die Schnittstelle zwischen IT-Dienstleistungen und Logistik zur Implementierung, Optimierung und Wartung der IdD-Technologien erheblich an Bedeutung gewinnen. Denn diese Aufgaben verlangen einerseits ein Wissen zur Strukturierung technischer Abläufe, Programmierung und Konfiguration von IT-Systemen und werden heute meist von (Fach-) Informatikern umgesetzt. Andererseits benötigen die IT-Spezialisten Detailkenntnisse über die logistischen Abläufe, um die IT-Systeme optimal in den Gesamtprozess integrieren zu können, wofür sie auf die betrieblichen Mitarbeiter und deren Prozesswissen zurückgreifen. Aber eine solche Entwicklung verlangt stellenweise ein stärkeres und detaillierteres Prozesswissen sowie ein höheres Planungswissen auf übergeordneten Ebenen. Dies könnte ein neues Aufgabenfeld für Planer (Prozessingenieure), Disponenten oder z. B. einen „Prozesscontroller“ eröffnen. Mögliche Profilinhalte werden in einer weitergehenden Veröffentlichung beschrieben.

\section{Schlussfolgerungen zur Mensch-Maschine-Interaktion}

Das „Internet der Dinge“ lässt die Komplexität der technischen Strukturen von Informations- und Kommunikationstechnik weiter steigen. Es werden die vernetzten Informationsobjekte, die als virtuelle Maschine angesehen werden können, mit dem Netz verbunden und zu Web-Services weiter entwickelt. Dadurch entsteht eine völlig neue Dimension von Maschinen im Internet, wenn dort auch noch Produkte angebunden werden. Aus dem Internet wird das „Internet der Dinge“ (vgl. Schmidt et al. 2008, S. 107f.). Mit Hilfe des "Internet der Dinge“ kann jeder Gegenstand mit digitalen Informationen aus dem Internet angereichert bzw. versorgt und damit auch gelenkt werden. Dieser Prozess schafft nicht nur neue ökonomische Werte, sondern wertet auch die Informationsbestände des Internets auf. Für Betriebe wird es letztendlich darauf ankommen, diese neuen Wertpotenziale zu erschließen und die dabei entstehenden Herausforderungen zu meistern (ebd., S.109).

Die Entwicklung hin zu autonom agierenden Systemen stellt die Mensch-Maschine-Beziehung auf dem Kopf. Eine weitgehende Implementierung dieser Technologie hat erhebliche Konsequenzen auf die Arbeitswelt. Dabei müssen zwei Zielrichtungen in Zukunft noch genauer betrachtet werden: 
- wie soll die Beziehung zwischen Mensch und IT-Systemen gestaltet werden, damit sich Menschen auf die von IT-Systemen generierten Situationen einstellen können,

- wie soll die Balance der Intelligenz zwischen Menschen und Maschinen austariert werden.

Die Studie zeigt, dass vor allem mithilfe von RFID-Technologien in Zukunft der Warenfluss immer mehr optimiert wird und die Rückverfolgbarkeit von Prozessen und Produkten erleichtert werden. Von einer Automatisierung ganzer Prozesse oder Prozessabschnitte ist man heute noch ein Stück entfernt. Man kann von teilautomatisierten Prozessen an der einen oder anderen Stelle sprechen. Dabei sind zwei Entwicklungsrichtungen für die Zukunft zu erkennen:

- Entwicklung von Expertensystemen mit Werkzeugcharakter für qualifizierte Fachkräfte (Werkzeugszenario),

- Einschränkung der Autonomie versierter Fachkräfte durch das Vordringen avancierter Technik bei Anlagen und Maschinen in der Logistik (Automatisierungsszenario).

Wenn die Entwicklung in Richtung Werkzeugszenario geht und der Mensch dabei die Gestaltungsfreiheit behält, kann die Technologie zu einer Art Assistenzsystem genutzt werden.

Ein Beispiel hierfür ist die Vereinfachung der Planungstätigkeit von Disponenten in der Spedition durch den Technologieeinsatz, die damit einhergehen könnte, dass die Berufskraftfahrer mit Hilfe der Technologie ausgewählte Disponententätigkeiten übernehmen können. Dabei begegnen sich Mensch und Maschine als handlungsmächtige Einheiten, die sich wechselseitig kontrollieren. Der Fahrer würde damit Aufgaben des Disponenten direkt beim Kunden vor Ort oder von unterwegs im Fahrzeug ausführen. Die Technik stellt ihm die entsprechenden Hilfsmittel zur Verfügung (Datenmasken, Auswahl der Fahraufträge). Er würde seine Aufträge nicht einfach nur abarbeiten, sondern je nach Situation in die Reihenfolge der Aufträge eingreifen. Damit kann er selbst auf Unwägbarkeiten im Straßenverkehr oder Wünsche von Kunden reagieren und seine Tour optimal gestalten. Gleichzeitig könnte die Technik ihn unterstützen, einen Auftrag eigenständig zu akquirieren.

Dagegen steht die Aussage, dass der „Fahrer vorwiegend funktionieren“ müsse: „Er soll nirgendwo gegenfahren, er soll nett zu den Kunden sein, er soll sich an seine Lenkzeiten halten" (Aussage eines Unternehmensleiters). Vorgaben durch die Disposition bzw. durch entsprechende technische Systeme sei Folge zu leisten, ein Mitdenken oder Diskutieren sei unerwünscht. Dieses wäre die Entwicklung in Richtung des Automatisierungsszenario. Die Technologie oder das „Internet der Dinge“ lenkt die Fachkräfte. Die Arbeit 
des IdD wird vollkommen technologisch gesteuert. Fachkräfte werden mit Informationen nicht versorgt und verfügen über keine Kompetenzen. Die entstehende Kompetenzlücke bedingt, dass nur eine vom IdD gelenkte Gestaltung von Arbeitsprozessen zielführend ist.

Nimmt man an, dass eine Entwicklung zu mehr (Teil)Automatisierung kurz- und mittelfristig stattfinden wird, dann wird sich das Aufgabenfeld der Beschäftigten verändern. Routinetätigkeiten werden zurückgehen, dafür werden sich die (schwierigen) Spezialfälle häufen. Zurückgeführt wird dies auf das sogenannte Automatisierungsparadoxon. In einem automatisierten System müssen die Beschäftigten nur noch in schwierigen Fällen eingreifen, dazu benötigen sie jedoch häufig eine höhere Qualifikation, die sie sich aber nicht mehr aufbauen können. Diese Sachlage, die in sogenannten High-TechFeldern (z.B. Flugzeuginstandsetzung) schon allgegenwärtig ist, breitet sich durch die Möglichkeiten umfangreicher Vernetzungen in alle Beschäftigungsfelder aus. Wie aus der Berufsbildung darauf zu reagieren ist, welche Strategien oder Konzepte als Antwort auf solche Entwicklungen stehen können, ist bisher eine offene Frage, die durch weitere Forschungsarbeiten zu klären sein wird.

\section{Literatur}

Becker, M.; Spöttl, G. (2008): Berufswissenschaftliche Forschung. Ein Arbeitsbuch für Studium und Praxis. Frankfurt a. M., et al. Peter Lang Verlag.

Brand, L.; Hülser, H., et al. (2009): Internet der Dinge - Perspektiven für die Logistik. Zukünftige Technologien Consulting Nr. 80.

Bullinger, H.-J.; Tombeil, A.-S. (2000): FreQueNz - ein Informationsnetz zur Früherkennung von Qualifikationserfordernissen. In: Bullinger, H.-J. (Hrsg.): Qualifikationen erkennen - Berufe gestalten. W. Bertelsmann Verlag. Bielefeld, S. 15-43.

Dworschak, B; Zaiser, H.; Martinetz, S.; Windelband, L. (2011): Logistik als Anwendungsfeld des „Internets der Dinge“ in der BMBF-Früherkennung. In: FreQueNz Zusammenfassung Internet der Dinge in der Logistik. Herausgegeben von FreQueNz, 2011, S. 2-4. http://www.frequenz.net/uploads/tx_freqprojerg/ Summary_logistik_final.pdf (Zugriff 23.02.2011).

Luhmann, N. (1990): Die Wissenschaft der Gesellschaft. Frankfurt am Main, Suhrkamp Verlag.

Schmidt, B. F.; Kündig, A.; Bütschi, D. (Hrsg.). (2008): Digitalisierte Wirtschaft: Praktisch kein Stein bleibt auf dem anderen. In: Die Verselbstständigung des Computers. Zürich. S. 99-116.

Schneider, S. (2009): RFID - einmal anders: Plagiatschutz mit EPC. Präsentation gehalten am 26.10.2009.

Ten Hompel, M. (2005): Das Internet der Dinge: Status, Perspektiven, Aspekte der aktuellen RFID-Entwicklung. Vortrag. Dortmunder Gespräche 2005: Fraunhofer Symposium RFID, Dortmund. 
Ten Hompel, M.; Bullinger, H.-J. (2007): Internet der Dinge. Berlin [et al.], Springer Verlag.

Weyer, J. (2007): Autonomie und Kontrolle. Arbeiten in hybriden Systemen am Beispiel der Luftfahrt. Technikfolgenabschätzung - Theorie und Praxis Nr. 2, 16. Jg., Dortmund.

Windelband, L. (2006): Früherkennung des Qualifikationsbedarfs in der Berufsbildung. Bielefeld, Bertelsmann Verlag.

Windelband, L.; Spöttl, G. (2003): Forschungshandbuch - Instrumente zur Früherkennung von Qualifizierungsbedarf. In: Papier 1. biat-Reihe Nr. 17. Flensburg.

Windelband, L.; et al. (2010): Abschlussbericht zur Studie „Qualifikationsanforderungen durch das Internet der Dinge in der Logistik" Bremen, Mai 2010. http://www.frequenz.net/uploads/tx_freqprojerg/Abschlussbericht_IdD_in_der_L ogistik_final.pdf (Zugriff 17.02.2011).

Windelband, L.; et al. (2011): Zukünftige Qualifikationsanforderungen durch das Internet der Dinge. Zusammenfassung. FreQueNz. Bonn: bmbf, 2011, S. 4-9.

Zahn, S. (2007): RFID in Bibliotheken. In: Band 16 von BIT online / Innovativ. Leinfelden-Echterdingen, B.I.T.Verlag. 



\section{Herausgeberschaft}

\section{Prof. Dr. Uwe Faßhauer}

Pädagogische Hochschule Schwäbisch Gmünd Institut für Bildung, Beruf und Technik

uwe.fasshauer@ph-gmuend.de

\section{Prof. Dr. Bärbel Fürstenau}

Technische Universität Dresden

Lehrstuhl für Wirtschaftspädagogik

baerbel.fuerstenau@tu-dresden.de

\section{Prof. Dr. Eveline Wuttke}

Goethe Universität Frankfurt

Professur für Wirtschaftspädagogik

wuttke@em.uni-frankfurt.de 



\section{Autorinnen und Autoren}

\section{Dr. Markus Ammann}

Universität Innsbruck

Institut für Organisation und Lernen

markus.ammann@uibk.ac.at

\section{Dr. Carmela Aprea}

Eidgenössisches Hochschulinstitut für Berufsbildung EHB Abteilung Forschung und Entwicklung carmela.aprea@iuffp-svizzera.ch

\section{Lic. Phil. I Christoph Arn}

Eidgenössisches Hochschulinstitut für Berufsbildung EHB Abteilungsverantwortlicher E-Learning christoph.arn@ehb-schweiz.ch

\section{Dr. Elena Boldrini}

Eidgenössisches Hochschulinstitut für Berufsbildung EHB Abteilung Forschung und Entwicklung elena.boldrini@iuffp-svizzera.ch

\section{Dr. Alberto Cattaneo}

Eidgenössisches Hochschulinstitut für Berufsbildung EHB Forschung und Entwicklung alberto.cattaneo(at)iuffp-svizzera.ch

\section{Dr. Alexandra Dehmel}

CEDEFOP - European Centre for the Development of Vocational Training, Griechenland

alexandra.dehmel@cedefop.europa.eu 


\section{Prof. em. Dr. Dres. H.c. Rolf Dubs}

Universität St. Gallen

Institut für Wirtschaftspädagogik

rolf.dubs@unisg.ch

\section{Dr. Uwe Elsholz}

Technische Universität Hamburg - Harburg

Institut für Technik, Arbeitsprozesse und Berufliche Bildung

Uwe.elsholz@tu-harburg.de

\section{Dr. Franz Kaiser}

Bundesinstitut für Berufsbildung

Abteilung 4: Ordnung der Berufsbildung

kaiser@bibb.de

\section{Dipl.-Hdl. Kristina Kögler}

Universität Bamberg

Lehrstuhl für Wirtschaftspädagogik

kristina.koegler@uni-bamberg.de

\section{Dr. Stephan Kösel}

Technische Universität Darmstadt

Institut für Berufspädagogik

koesel@bpaed.tu-darmstadt.de

\section{Dipl.-Päd. Petra Köster}

Universität Trier

Abteilung Weiterbildung

koesterp@uni-trier.de

\section{Dr. Martin Kröll}

Ruhr Universität Bochum

Institut für Arbeitswissenschaft

martin.kroell@rub.de 


\section{Dip.-Hdl. Birgit Lehmann}

Universität Mannheim

Lehrstuhl für Wirtschaftspädagogik

lehmann@bwl.uni-mannheim.de

\section{Prof. Dr. Rita Meyer}

Universität Trier

Abteilung Weiterbildung

rmeyer@uni-trier.de

\section{Elisa Motta, MSc}

Eidgenössisches Hochschulinstitut für Berufsbildung EHB Abteilung Forschung und Entwicklung elisa.motta@iuffp-svizzera.ch

\section{Dipl.-Päd. Julia Müller}

Universität Trier

Abteilung Weiterbildung

jkm@uni-trier.de

\section{Dipl.-Hdl. Jeannine Ryssel}

Technische Universität Dresden

Lehrstuhl Wirtschaftspädagogik

jeannine.ryssel@tu-dresden.de

\section{Lic. Phil. I Alicja Sroka}

Eidgenössisches Hochschulinstitut für Berufsbildung EHB

Forschung und Entwicklung

alicja.sroka@ehb-schweiz.ch

\section{Prof. Dr. Georg Spöttl}

Universität Bremen

Institut Technik und Bildung Bremen

spoettl@uni-bremen.de 


\section{Dr. Georg Tafner}

Karl-Franzens-Universität Graz

Institut für Wirtschaftspädagogik

georg.tafner@uni-graz.at

\section{Dr. Sandra Trost, MBR}

Ludwig-Maximilians-Universität München

Institut für Wirtschaftspädagogik

trost@bwl.lmu.de

\section{Dr. Lars Windelband}

Universität Magdeburg

Institut für Berufs- und Betriebspädagogik

lars.windelband@ovgu.de

\section{Prof. Dr. Eveline Wuttke}

Goethe Universität Frankfurt am Main

Professur für Wirtschaftspädagogik-

Empirische Lehr-Lern-Forschung

wuttke@em.uni-frankfurt.de 
Schriftenreihe der Sektion Berufs- und Wirtschaftspädagogik der Deutschen Gesellschaft für Erziehungswissenschaft (DGfE)

D. MünK, J. VAn Buer, K. Breuer, T. DEIßINGER (HRSG.)

E. WUtTKe, M. Friese, Hundert Jahre kaufmännische Ausbildung in Berlin 2007. 264 S. Kt. $24,90 €(D), 25,60 €(A), 44,00 \mathrm{SFr}$ ISBN 978-3-86649-118-2

B. Fürstenau, R. TEnberg (HRsg.) Dimensionen der Berufsbildung 2010. 186 S. Kt. $24,90 €(D), 25,60 €(A), 35,90 \mathrm{SFr}$ ISBN 978-3-86649-316-2

D. MüNK, T. DEIßINGER, R. TENBERg (HRSG.)

U. FAßhAuER, J. AfF, B. Fürstenau, E. WUTTKE (HRSG.) Lehr-Lernforschung und Profes-

Forschungserträge aus der Berufs- sionalisierung und Wirtschaftspädagogik 2009. 197 S. Kt. $24,90 €(D), 25,60 €(A), 44,00 \mathrm{SFr}$ ISBN 978-3-86649-284-4

D. MÜnK, P. Gonon, K. BREUER, T. DEIßINGER (HRSG.) 2011. 246 S. Kt. $29,90 €(D), 30,80 €(A), 41,90 \mathrm{SFr}$ ISBN 978-3-86649-367-4

Auch im Open Access erhältlich auf: http://www.budrich-verlag.de

Modernisierung der Berufsbildung 2008. 227 S. Kt.

$24,90 €(D), 25,60 €(A), 44,00 \mathrm{SFr}$ ISBN 978-3-86649-167-0

U. FAßHAUER, BÄRBERL FÜRSTENAU, Eveline WutTKE (HRSG.)

Grundlagenforschung zum Dualen System und Kompetenzentwicklung in der Lehrerbildung 2012. 169 S. Kt.

E. WuttKe, H. Ebner, B. Fürstenau, R. TEnBERg (HRSg.) $29,90 €(D), 30,80 €(A), 41,90 \mathrm{SFr}$ ISBN 978-3-86649-461-9

Erträge und Perspektiven berufs- Auch im Open Access erhältlich unter und wirtschaftspädagogischer Forschung 2009. 171 S. Kt. $19,90 €(D), 20,50 €(A), 35,90 \mathrm{SFr}$ ISBN 978-3-86649-277-6 http://www.budrich-verlag.de

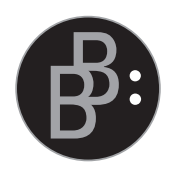
Verlag Barbara Budrich • Barbara Budrich Publishers Stauffenbergstr. 7. D-51379 Leverkusen Opladen Tel +49 (0)2171.344.594 • Fax +49 (0)2171.344.693 • info@budrich.de

Weitere Bücher und Zeitschriften unter www.budrich-verlag.de 


\section{Kinder, Teenies und Jugendliche und ihre Peers}

Heinz-Hermann Krüger

Aline Deinert

Maren Zschach

Jugendliche und ihre Peers

Freundschaftsbeziehungen und Bildungsbiografien in einer Längsschnittperspektive

2012. 292 Seiten. Kart.

$29,90 €(D), 30,80 €(A), 41,90 \mathrm{SFr}$

ISBN 978-3-86649-460-2
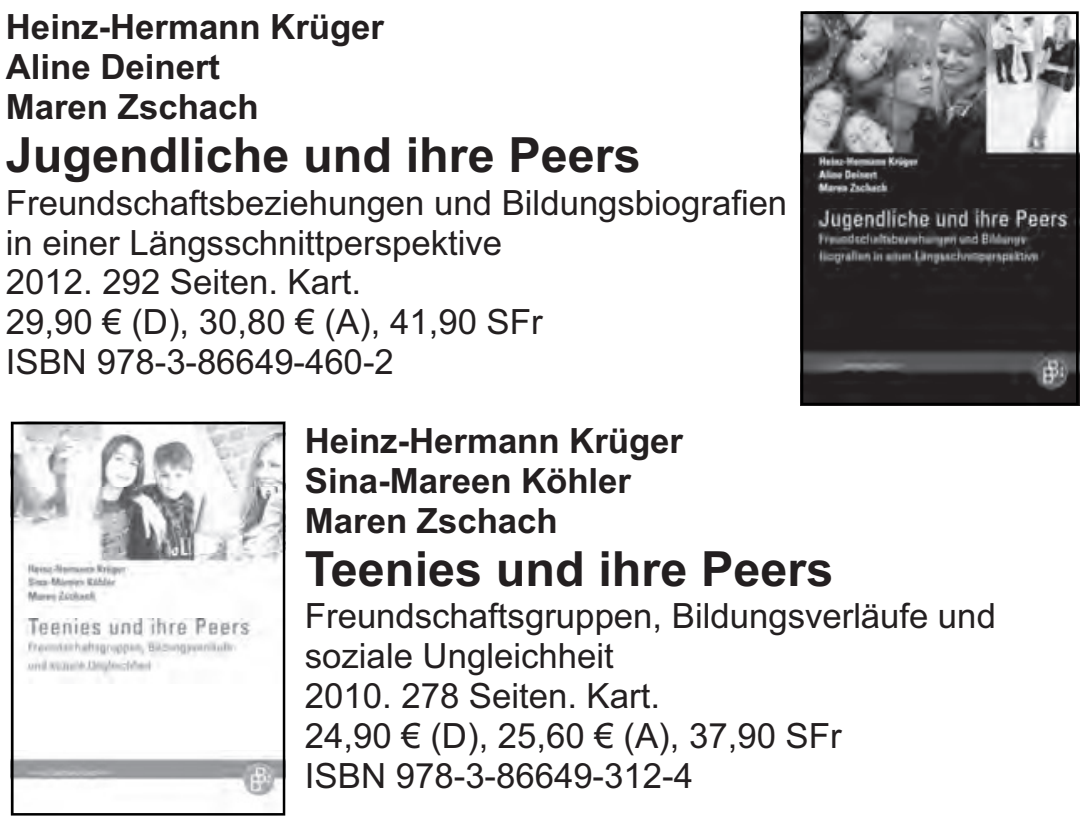

Heinz-Hermann Krüger

Sina-Mareen Köhler

Maren Zschach

Teenies und ihre Peers

Freundschaftsgruppen, Bildungsverläufe und soziale Ungleichheit 2010. 278 Seiten. Kart.

$24,90 €(D), 25,60 €(A), 37,90 \mathrm{SFr}$ ISBN 978-3-86649-312-4

Heinz-Hermann Krüger

Sina-Mareen Köhler

Maren Zschach

Nicolle Pfaff

\section{Kinder und ihre Peers}

Freundschaftsbeziehungen und schulische

Bildungsbiographien

2008. 317 Seiten.

$26,90 €(D), 27,70 €(A), 47,00 \mathrm{SFr}$

ISBN 978-3-86649-114-4

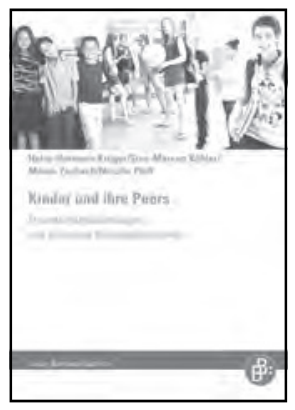

Wissen, was läuft: Kostenlos budrich intern abonnieren!

Formlose eMail an: info@budrich.de - Betreff: budrich intern

www.budrich-verlag.de • www.budrich-journals.de 